\title{
DEVELOPMENT AND EXPERIMENTAL BENCHMARKING OF NUMERIC FLUID STRUCTURE INTERACTION MODELS FOR RESEARCH REACTOR FUEL ANALYSIS
}

\author{
A Dissertation presented to \\ the Faculty of the Graduate School \\ University of Missouri
}

\author{
In Partial Fulfillment \\ of the Requirements for the Degree \\ Doctor of Philosophy
}

By

JOHN C. KENNEDY

Dr. Gary L. Solbrekken, Dissertation Supervisor

DECEMBER 2015 
(C) Copyright by John Kennedy 2015

All Rights Reserved 
The undersigned, appointed by the Dean of the Graduate School, have examined the dissertation entitled

\section{DEVELOPMENT AND EXPERIMENTAL BENCHMARKING OF NUMERIC FLUID STRUCTURE INTERACTION MODELS FOR RESEARCH REACTOR FUEL ANALYSIS}

Presented by John C. Kennedy

A candidate for the degree Doctor of Philosophy

And hereby certify that in their opinion it is worthy of acceptance

Dr. Gary L. Solbrekken

Dr. Robert Winholtz

Dr. C.L. Chen

Dr. Frank Feng

Dr. Hani Salim 


\section{ACKNOWLEDGEMENTS}

When starting on this project in 2009 , I had no idea where it would lead or what I would learn along the way. What initially started as a desire to get a quick Master's degree quickly grew into much more. I've been amazed by my experiences the past six years, and more importantly have been fortunate to meet and work with some amazing individuals. In particular, I owe a great debt of gratitude to many individuals at Argonne National Laboratory and the University of Missouri.

I'd like to thank a few of the great people who I shared a small, cramped, windowless lab with during this process: Kyler Turner, Philip Makarewicz, Srisharan Govindarajan, and Casey Jesse. I learned more from these individuals than I think I'll ever realize and owe them more than I could ever repay. Their wisdom, patience, and most importantly humor, made the long days, late nights, and stressful deadlines a bit more bearable.

I'd also like to thank my advisor, Dr. Gary Solbrekken. He has been an invaluable mentor during this process. Without his support and guidance none of the work and progress presented in this dissertation would have been possible.

Finally, to my family. My parents, John and Lisa, and my brother Josh. They've borne the brunt of the moments of greatest frustration and doubt over these past 6 years. Without their continued support, encouragement, and occasional pep-talk, I would have walked away from this long ago.

Again, thank you to everyone helped with this project and helped me personally. 


\section{TABLE OF CONTENTS}

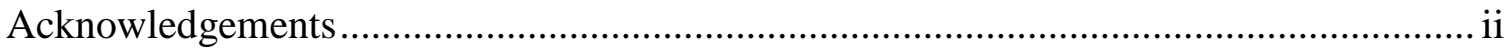

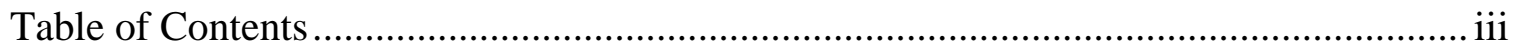

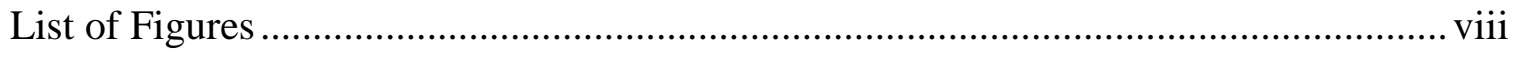

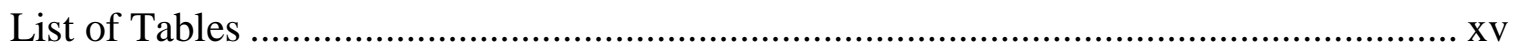

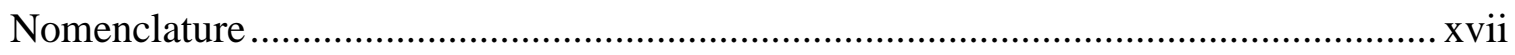

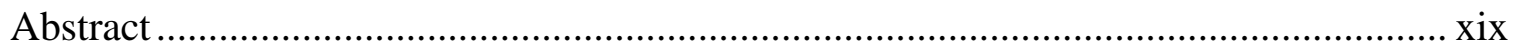

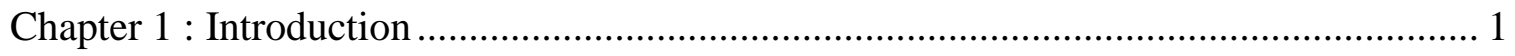

1.1 : Reactor Core Design............................................................................. 1

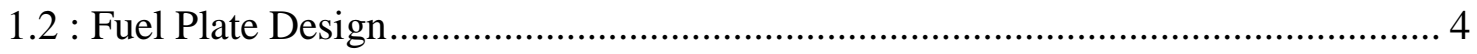

1.3 : Motivation and Reactor Conversion............................................................. 5

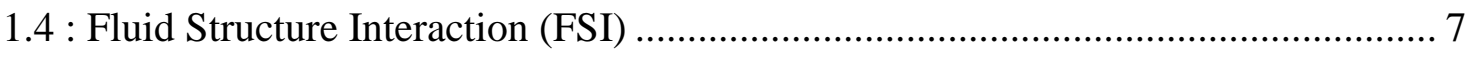

Chapter 2 : Background \& Literature Review ...................................................... 9

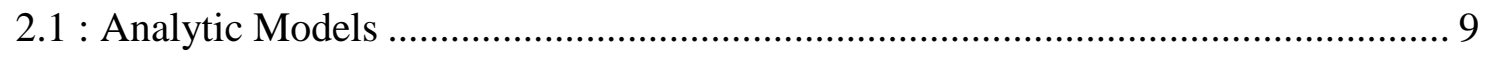

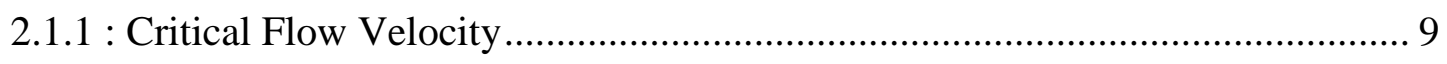

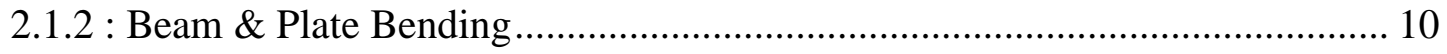

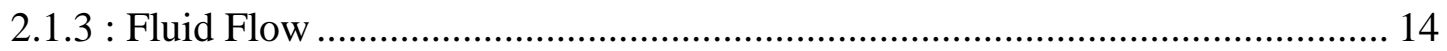

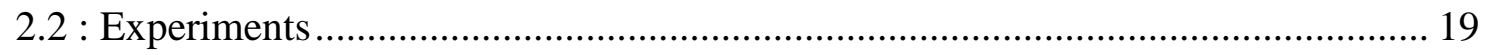

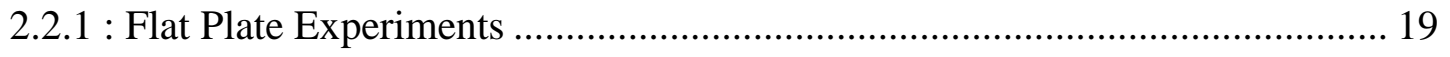

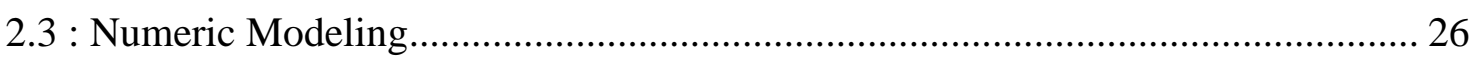

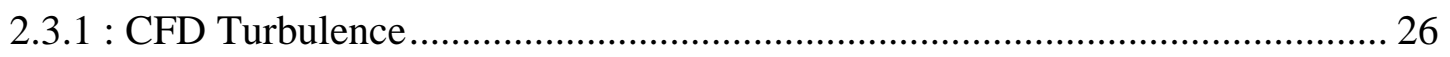

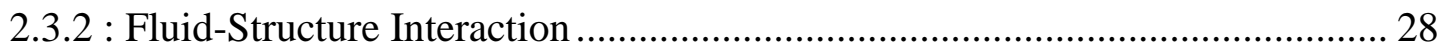

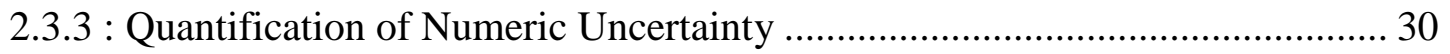


Chapter 3 : Hydro-Mechanical Flow Loop ……………................................................. 33

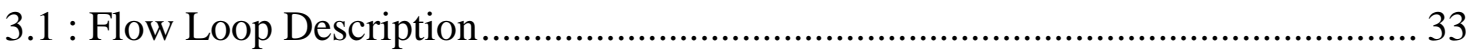

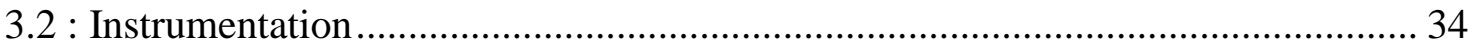

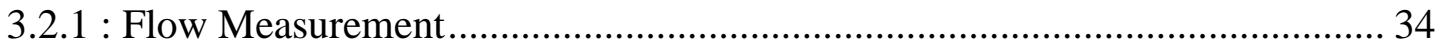

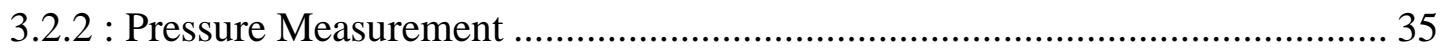

3.2.3 : Laser Based Channel Measurement ................................................................. 35

3.3 : Data Acquisition \& Control System.................................................................... 38

Chapter 4 : Experiment Setup …………………………….................................... 42

4.1 : Experiment Objective .................................................................................. 42

4.2 : Test Section Design ......................................................................................... 44

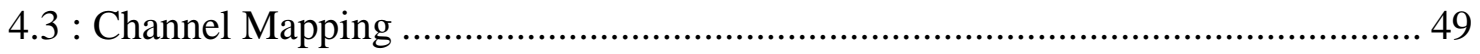

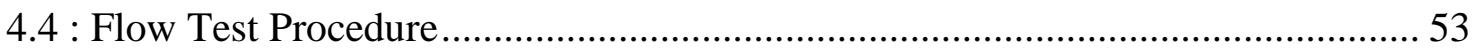

Chapter 5 : Experiment Results ............................................................................... 57

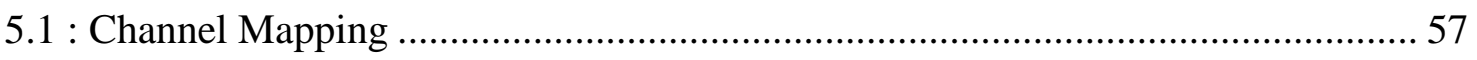

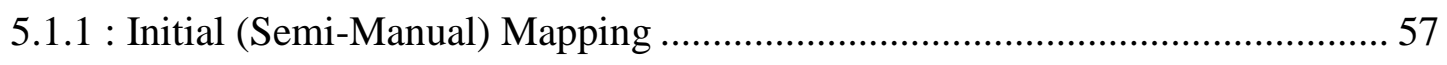

5.1.2 : Upgraded (Fully Automatic) Mapping ………….............................................. 62

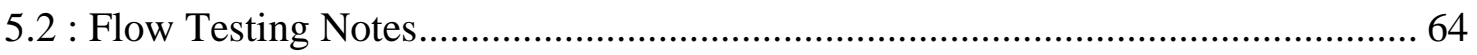

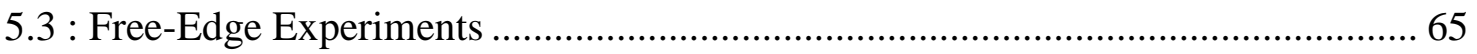

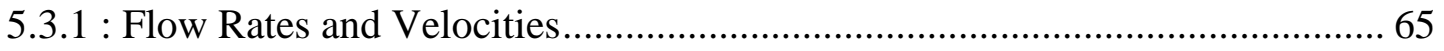

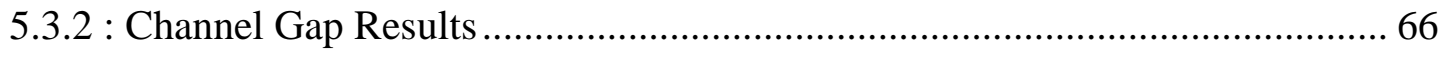

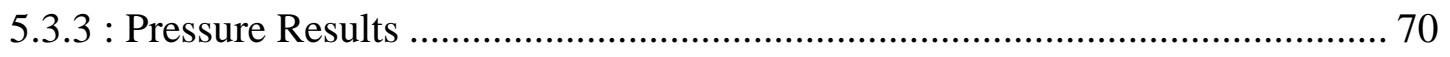

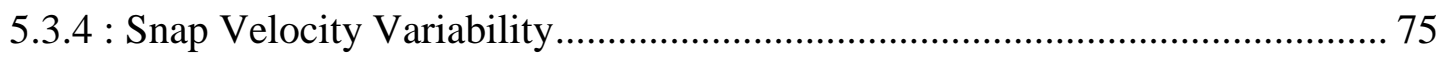

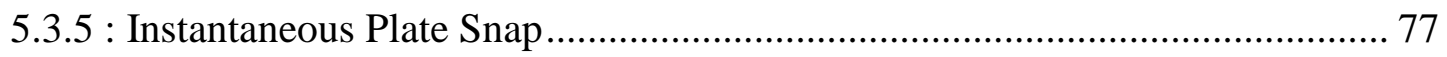

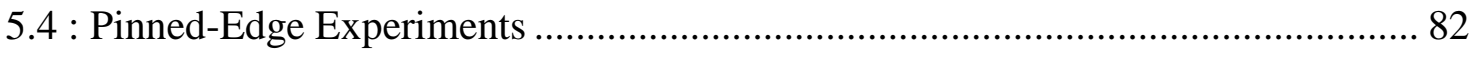


5.4.1 : Flow Rates and Velocities.................................................................. 82

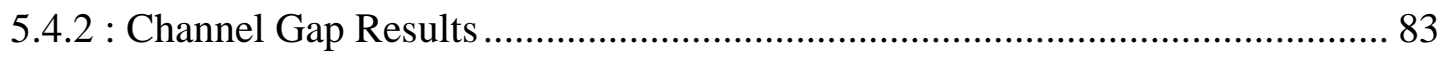

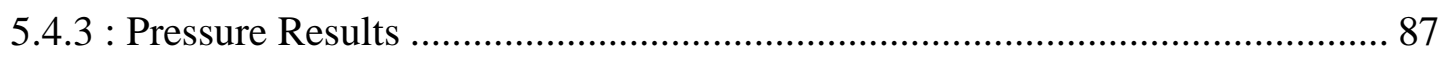

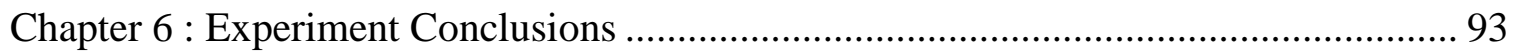

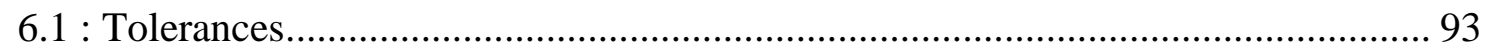

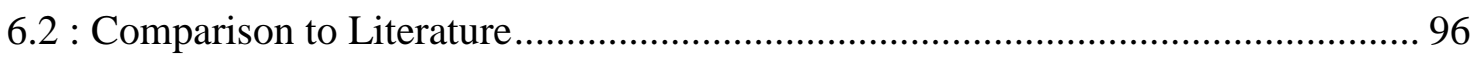

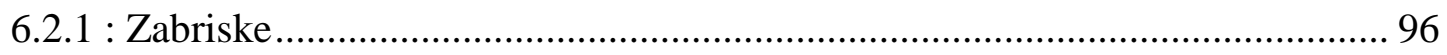

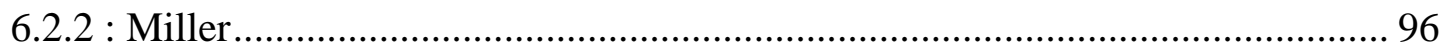

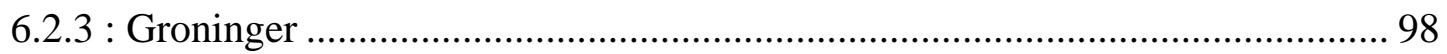

6.3 : Effect of Channel Variation on Expected Deflection....................................... 99

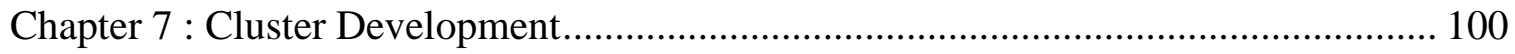

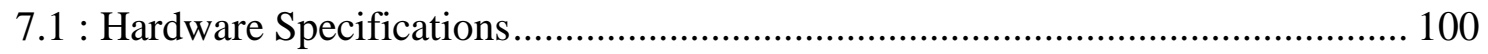

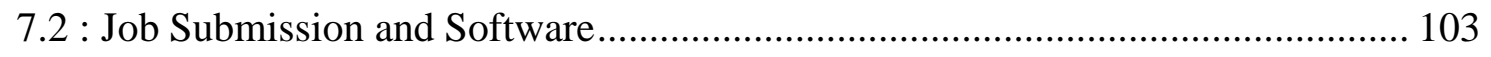

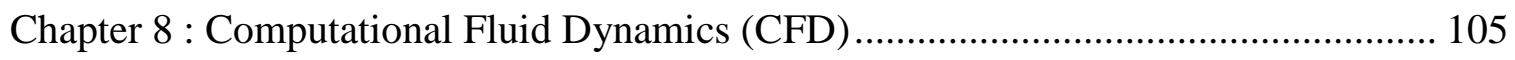

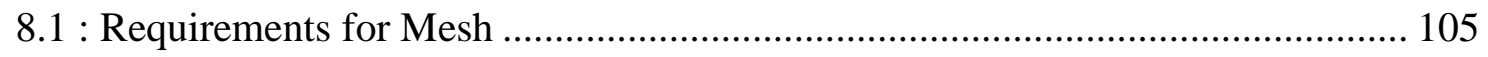

8.2 : Turbulence and Mesh Validation Study ...................................................... 106

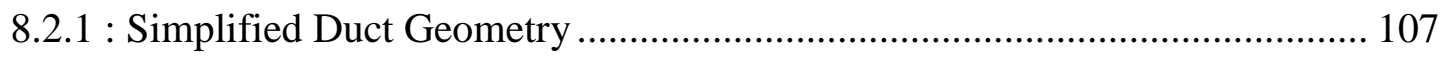

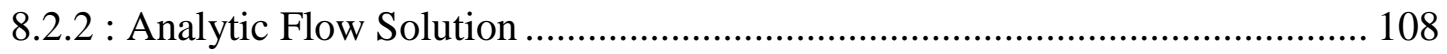

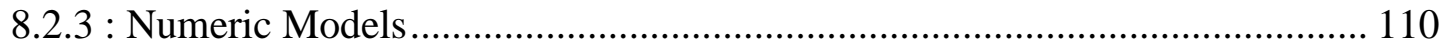

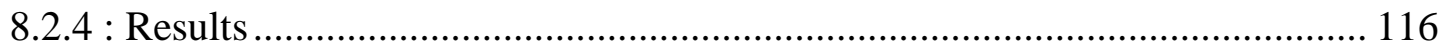

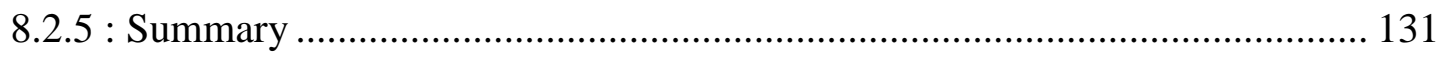

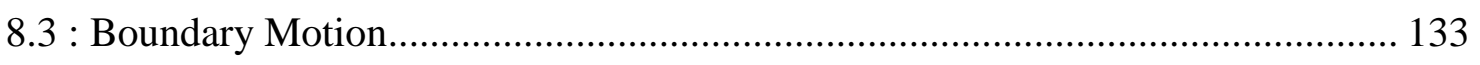

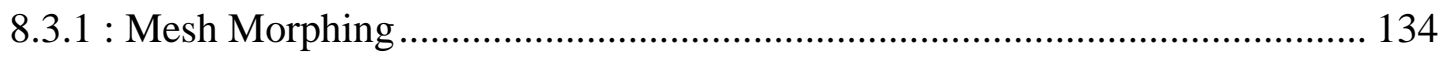

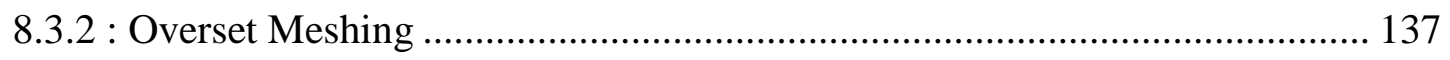


8.4 : Final Fluid Model

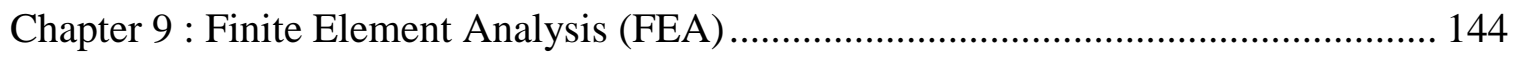

9.1 : Plate Model.............................................................................................. 144

9.2 : Element Type and Mesh ..................................................................... 146

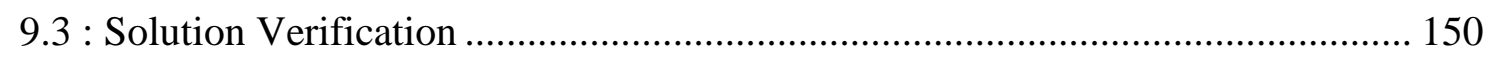

Chapter 10 : Numeric Fluid Structure Interaction (FSI) ........................................ 153

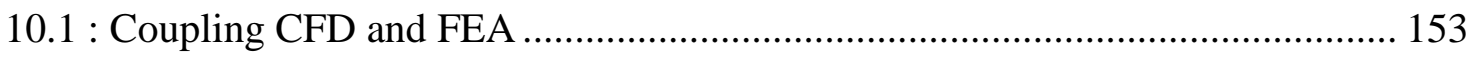

10.1 .1 : Data Coupling Methods ....................................................................... 153

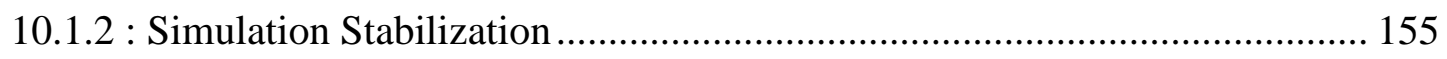

10.2 : Base (Ideal) Geometry Simulations......................................................... 157

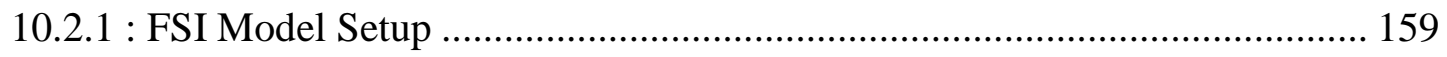

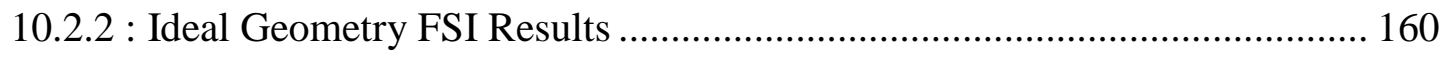

Chapter 11 : As-Built Numeric FSI Simulations .................................................... 165

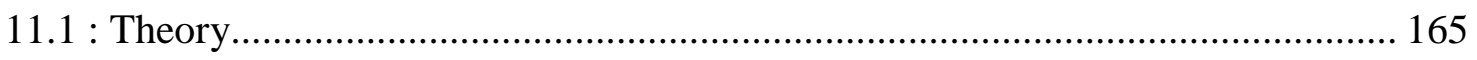

11.2 : CFD Background Mesh Modification ..................................................... 165

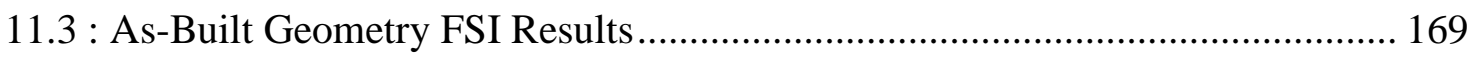

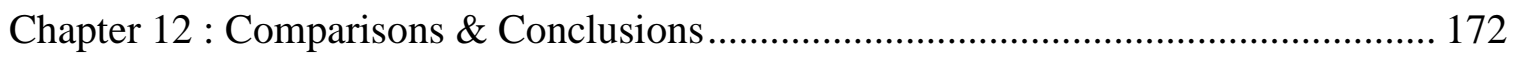

12.1 : Deflection Results Comparison ................................................................ 172

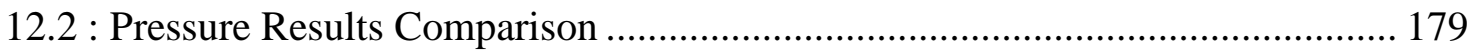

12.3 : Additional Boundary Condition Improvements ........................................ 186

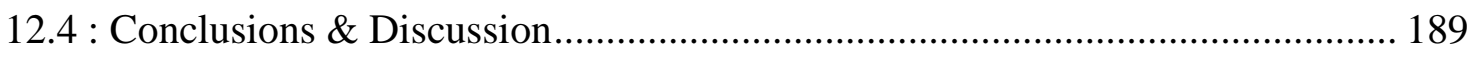

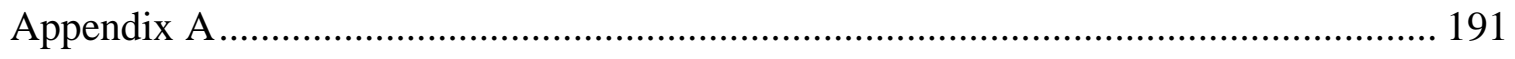

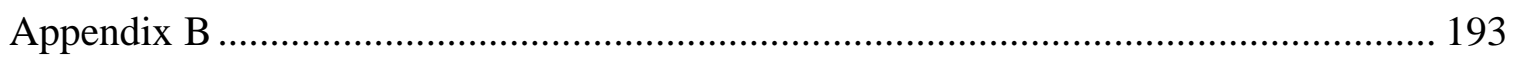

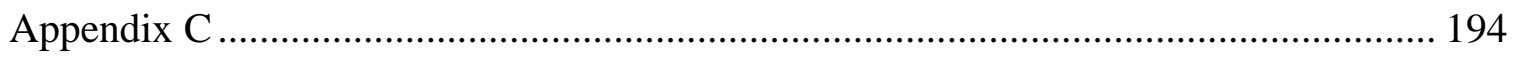




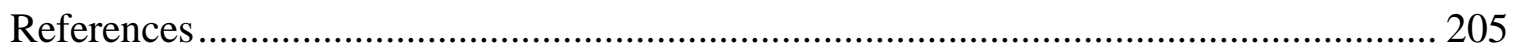

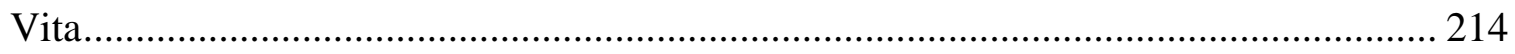




\section{LIST OF FIGURES}

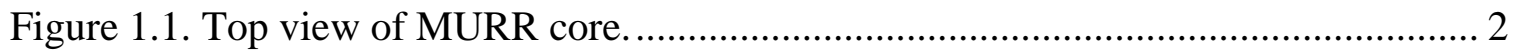

Figure 1.2. MURR fuel element with 24 fuel plates................................................... 3

Figure 1.3. MURR fuel element end cap and plates.............................................. 3

Figure 1.4. Cross section schematic of $\mathrm{U}-\mathrm{Al}$ dispersion fuel plate .............................. 4

Figure 1.5. Cross section schematic of LEU fuel plate............................................ 5

Figure 2.1. Deflection of plate (long beam) under uniform load with two opposite fixed

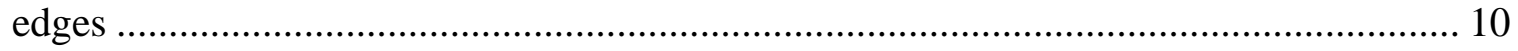

Figure 2.2. Simple beam deflection vs. Timoshenko plate deflection.......................... 13

Figure 2.3. Conversion of a composite plate if Young's Moduli $\mathrm{E}_{\mathrm{A}}$ and $\mathrm{E}_{\mathrm{B}}$ to a

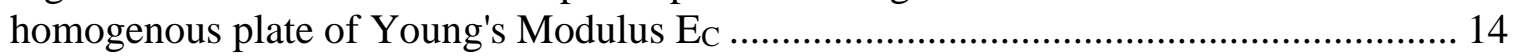

Figure 2.4. Axial fluid resistance diagram around a deflected plate .......................... 16

Figure 2.5. Star-CCM+ overset grid interpolation method ......................................... 29

Figure 3.1. Hydro-Mechanical Flow Loop as of July 2014 ...................................... 34

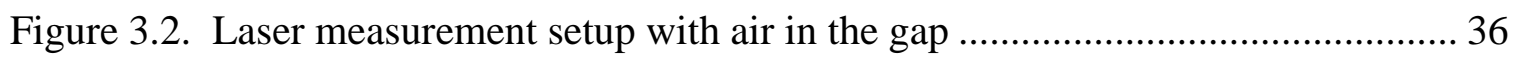

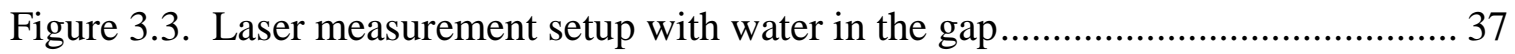

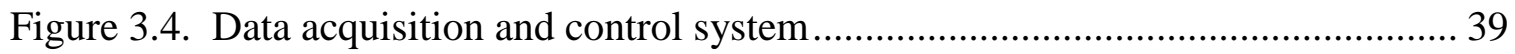

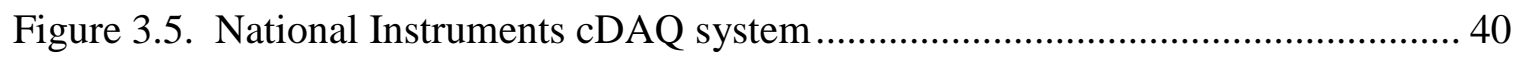

Figure 3.6. LabView graphical user interface ................................................... 41

Figure 4.1. Overview of laser positioning and flow loop structural changes ................ 44

Figure 4.2. Test section horizontal cross-section (to scale) .................................... 45

Figure 4.3. Test section vertical (axial) cross-section (not to scale)............................ 46

Figure 4.4. Test section ready for flow testing ................................................... 48

Figure 4.5. Ideal channel thickness vs. actual thickness ........................................... 49

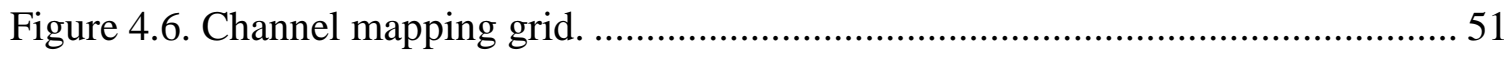




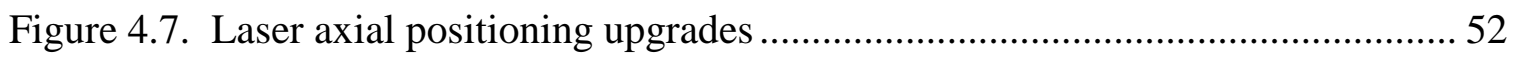

Figure 5.1. Channel gap mean, minimum, and maximum values................................ 58

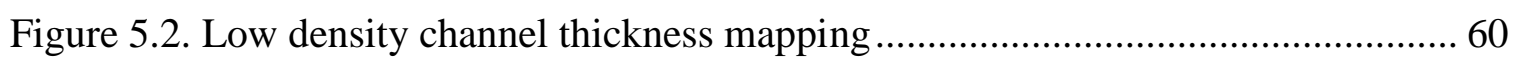

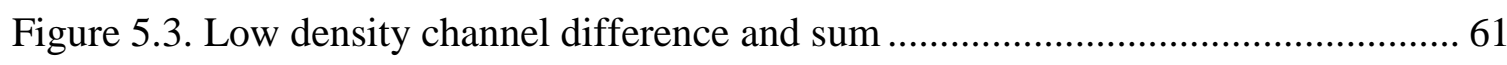

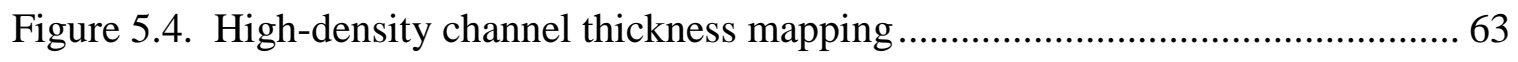

Figure 5.5. Channel gap and deflection at location $\mathrm{A}-12.7 \mathrm{~mm}$ from trailing edge with

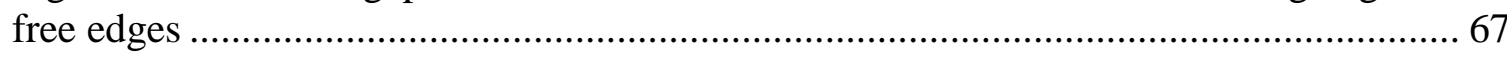

Figure 5.6. Channel gap and deflection at location $\mathrm{B}-152.4 \mathrm{~mm}$ from trailing edge with

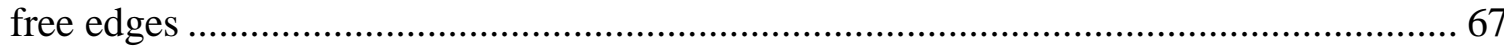

Figure 5.7. Channel gap and deflection at location $\mathrm{C}-226.7 \mathrm{~mm}$ from trailing edge with

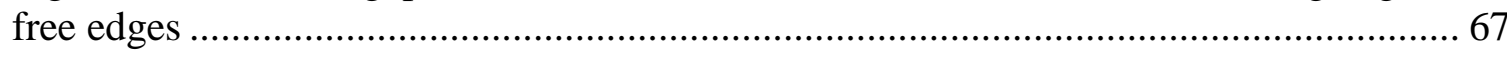

Figure 5.8. Channel gap and deflection at location D - $290.5 \mathrm{~mm}$ from trailing edge with

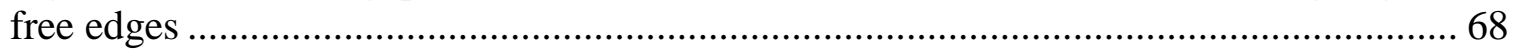

Figure 5.9. Channel gap and deflection at location $\mathrm{E}-474.3 \mathrm{~mm}$ from trailing edge with

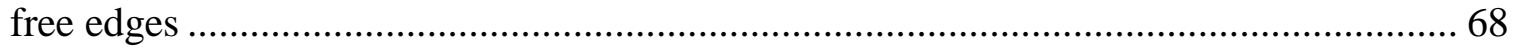

Figure 5.10. Channel gap and deflection at location $\mathrm{F}-550.5 \mathrm{~mm}$ from trailing edge

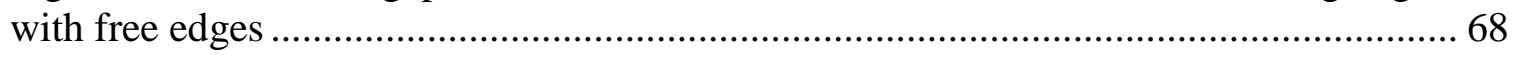

Figure 5.11. Channel gap and deflection at location $\mathrm{G}-635.0 \mathrm{~mm}$ from trailing edge

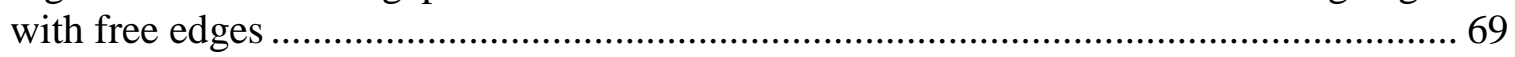

Figure 5.12. Plate profiles at various flow rates with free edges ................................ 70

Figure 5.13. Channel pressure difference in free edge experiments, $64.77 \mathrm{~mm} \mathrm{(2.55")}$

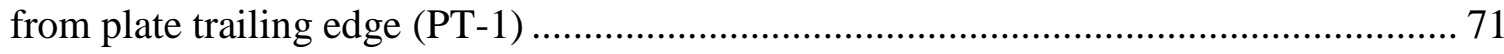

Figure 5.14. Channel pressure difference in free edge experiments, $129.54 \mathrm{~mm}$ (5.10")

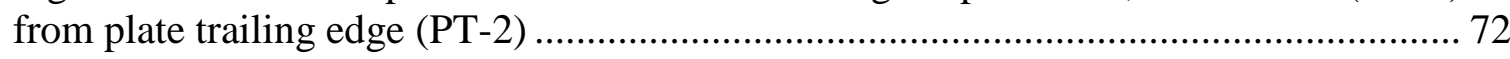

Figure 5.15. Channel pressure difference in free edge experiments, $194.31 \mathrm{~mm}$ (7.65")

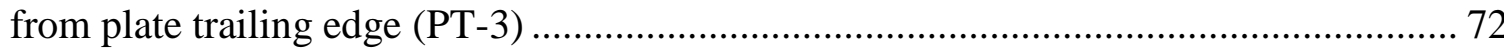

Figure 5.16. Channel pressure difference in free edge experiments, $259.08 \mathrm{~mm}(10.20 ")$ from plate trailing edge (PT-4) ....................................................................... 73

Figure 5.17. Channel pressure difference in free edge experiments, $323.85 \mathrm{~mm}(12.75 ")$ from plate trailing edge (PT-5) 73 
Figure 5.18. Channel pressure difference in free edge experiments, $388.62 \mathrm{~mm}(15.30 ")$

from plate trailing edge (PT-6)

Figure 5.19. Channel pressure difference in free edge experiments, $453.39 \mathrm{~mm}(17.85 ")$ from plate trailing edge (PT-7)

Figure 5.20. Channel pressure difference in free edge experiments, $582.93 \mathrm{~mm}(22.95 ")$

from plate trailing edge (PT-9)

Figure 5.21. Change in channel gap during plate snap at axial location E and flow rate of $2.927 \mathrm{~kg} / \mathrm{s}$. 78

Figure 5.22. Pressure difference change during plate snap with lasers at axial location E and flow rate of $2.927 \mathrm{~kg} / \mathrm{s}$. 78

Figure 5.23. Change in smaller channel gap during plate snap at axial location D and flow rate $2.936 \mathrm{~kg} / \mathrm{s}$

Figure 5.24. Pressure difference change during plate snap with lasers at axial location D and flow velocity of $2.936 \mathrm{~kg} / \mathrm{s}$ 80

Figure 5.25. Pressure profiles. Blue lines are pre-snap data sets, orange lines are postsnap data sets. Positive pressure indicates higher pressure in the smaller channel ......... 81

Figure 5.26. Channel gap and deflection at location A - $12.7 \mathrm{~mm}$ from trailing edge with pinned edges

Figure 5.27. Channel gap and deflection at location B $-152.4 \mathrm{~mm}$ from trailing edge with pinned edges 84

Figure 5.28. Channel gap and deflection at location $\mathrm{C}-226.7 \mathrm{~mm}$ from trailing edge with pinned edges

Figure 5.29. Channel gap and deflection at location D - $290.5 \mathrm{~mm}$ from trailing edge with pinned edges

Figure 5.30. Channel gap and deflection at location $\mathrm{E}-474.3 \mathrm{~mm}$ from trailing edge with pinned edges

Figure 5.31. Channel gap and deflection at location $\mathrm{F}-550.5 \mathrm{~mm}$ from trailing edge with pinned edges

Figure 5.32. Channel gap and deflection at location $\mathrm{G}-635.0 \mathrm{~mm}$ from trailing edge with pinned edges 86

Figure 5.33. Plate profiles at various flow rates with pinned edges. 87 
Figure 5.34. Channel pressure difference in pinned edge experiments, $64.77 \mathrm{~mm}(2.55 ")$

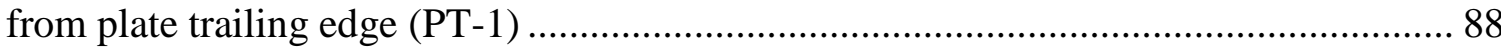

Figure 5.35. Channel pressure difference in pinned edge experiments, $129.54 \mathrm{~mm}\left(5.10^{\prime \prime}\right)$ from plate trailing edge (PT-2) 89

Figure 5.36. Channel pressure difference in pinned edge experiments, $194.31 \mathrm{~mm}(7.65 ")$ from plate trailing edge (PT-3)

Figure 5.37. Channel pressure difference in pinned edge experiments, $259.08 \mathrm{~mm}$ $(10.20 ")$ from plate trailing edge (PT-4). 90

Figure 5.38. Channel pressure difference in pinned edge experiments, $323.85 \mathrm{~mm}$ (12.75") from plate trailing edge (PT-5). 90

Figure 5.39. Channel pressure difference in pinned edge experiments, $388.62 \mathrm{~mm}$ (15.30") from plate trailing edge (PT-6).

Figure 5.40. Channel pressure difference in pinned edge experiments, $453.39 \mathrm{~mm}$ (17.85") from plate trailing edge (PT-7)

Figure 5.41. Channel pressure difference in pinned edge experiments, $582.93 \mathrm{~mm}$ (22.95") from plate trailing edge (PT-9)

Figure 6.1. Cross sectional view of proposed MURR LEU plate. Provided as Figure 1 in reference [46] 95

Figure 6.2. Channel gap vs. fraction of Miller Critical Velocity at $635 \mathrm{~mm}$ (25") from the trailing edge with free leading and trailing edges..... 98

Figure 7.1. Mini high performance compute cluster (HPCC). ................................ 100

Figure 7.2. Detailed lab network and cluster map ............................................... 102

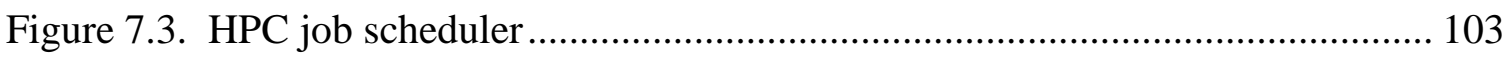

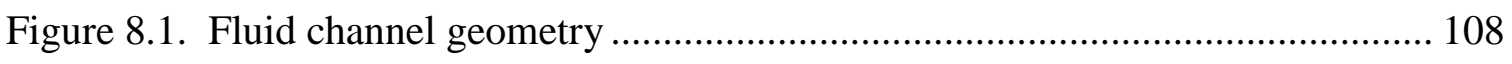

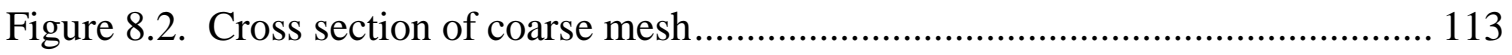

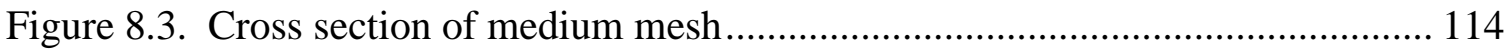

Figure 8.4. Cross section of fine mesh with close-up of near wall mesh .................... 115

Figure 8.5. RMS pressure drop error for all models ............................................ 120

Figure 8.6. RMS wall shear stress error for all models ........................................ 124 


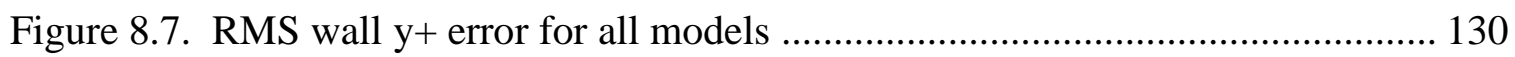

Figure 8.8. CFD mesh before and after morphing ..................................................... 135

Figure 8.9. Low quality mesh resulting from repeated morphing ............................... 136

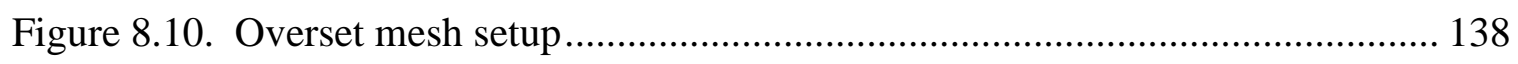

Figure 8.11. CFD model front view ..................................................................... 140

Figure 8.12. Overset mesh cross-section and interpolation .......................................... 141

Figure 8.13. Near wall meshes.............................................................................. 142

Figure 9.1. FEA model plate geometry .................................................................. 145

Figure 9.2. Close up of plate meshes. C3D8I on left, SC8R on right........................... 146

Figure 9.3. C3D8I, NLGeom off, axial deflection profiles under uniform pressure loads

Figure 9.4. SC8R, NLGeom off, axial deflection profiles under uniform pressure loads

Figure 9.5. C3D8I, NLGeom on, axial deflection profiles under uniform pressure loads 149

Figure 9.6. SC8R, NLGeom on, axial deflection profiles under uniform pressure loads

Figure 9.7. FEA comparison to analytic …………………....................................... 151

Figure 10.1. FSI Co-Simulation coupling process...................................................... 154

Figure 10.2. FSI geometry: Plate (green mesh); Background fluid (blue mesh); Overset

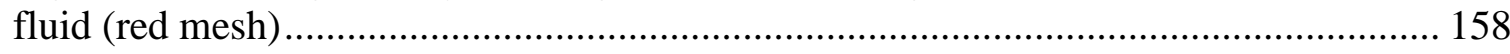

Figure 10.3. $3.43 \mathrm{~kg} / \mathrm{s}$ Abaqus deflection profile...................................................... 161

Figure 10.4. Axial deflection profiles for Ideal geometry FSI simulations ................... 162

Figure 10.5. Channel pressure data at $3.43 \mathrm{~kg} / \mathrm{s}$ flow .................................................. 163

Figure 10.6. Pressure force acting on plate for Ideal geometry simulations.................. 164

Figure 11.1. Resulting channel thickness after morphing outer fluid walls .................. 168

Figure 11.2. Axial deflection profiles for As-Built geometry FSI simulations ............. 170 
Figure 11.3. Axial pressure profiles for As-Built FSI simulations 171

Figure 12.1. $1.04 \mathrm{~kg} / \mathrm{s}$ experiment \& FSI axial deflection results comparison............. 173

Figure 12.2. $1.40 \mathrm{~kg} / \mathrm{s}$ experiment \& FSI axial deflection results comparison.............. 173

Figure 12.3. $1.74 \mathrm{~kg} / \mathrm{s}$ experiment \& FSI axial deflection results comparison.............. 174

Figure 12.4. $2.30 \mathrm{~kg} / \mathrm{s}$ experiment \& FSI axial deflection results comparison.............. 174

Figure 12.5. $2.60 \mathrm{~kg} / \mathrm{s}$ experiment \& FSI axial deflection results comparison.............. 175

Figure 12.6. $2.76 \mathrm{~kg} / \mathrm{s}$ experiment \& FSI axial deflection results comparison.............. 175

Figure 12.7. $2.86 \mathrm{~kg} / \mathrm{s}$ experiment \& FSI axial deflection results comparison.............. 176

Figure 12.8. $2.95 \mathrm{~kg} / \mathrm{s}$ experiment \& FSI axial deflection results comparison.............. 176

Figure 12.9. $3.43 \mathrm{~kg} / \mathrm{s}$ experiment \& FSI axial deflection results comparison.............. 177

Figure 12.10. $3.91 \mathrm{~kg} / \mathrm{s}$ experiment \& FSI axial deflection results comparison............ 177

Figure 12.11. $4.34 \mathrm{~kg} / \mathrm{s}$ experiment \& FSI axial deflection results comparison............ 178

Figure 12.12. $1.04 \mathrm{~kg} / \mathrm{s}$ experiment \& FSI axial pressure results comparison ............. 180

Figure 12.13. $1.40 \mathrm{~kg} / \mathrm{s}$ experiment \& FSI axial pressure results comparison ............. 180

Figure 12.14. $1.74 \mathrm{~kg} / \mathrm{s}$ experiment \& FSI axial pressure results comparison .............. 181

Figure $12.15 .2 .30 \mathrm{~kg} / \mathrm{s}$ experiment \& FSI axial pressure results comparison .............. 181

Figure 12.16. $2.60 \mathrm{~kg} / \mathrm{s}$ experiment \& FSI axial pressure results comparison ............. 182

Figure 12.17. $2.76 \mathrm{~kg} / \mathrm{s}$ experiment \& FSI axial pressure results comparison ............. 182

Figure 12.18. $2.86 \mathrm{~kg} / \mathrm{s}$ experiment \& FSI axial pressure results comparison ............. 183

Figure 12.19. $2.95 \mathrm{~kg} / \mathrm{s}$ experiment \& FSI axial pressure results comparison ............. 183

Figure 12.20. $3.43 \mathrm{~kg} / \mathrm{s}$ experiment \& FSI axial pressure results comparison ............. 184

Figure 12.21. $3.91 \mathrm{~kg} / \mathrm{s}$ experiment \& FSI axial pressure results comparison .............. 184

Figure 12.22. $4.34 \mathrm{~kg} / \mathrm{s}$ experiment \& FSI axial pressure results comparison .............. 185

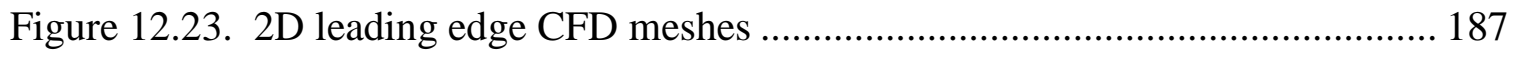




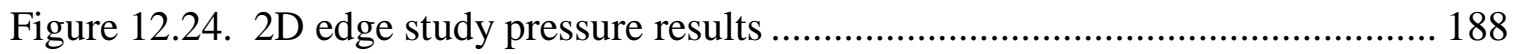




\section{LIST OF TABLES}

Table 1.1. Selected material properties for LEU fuel components ................................ 7

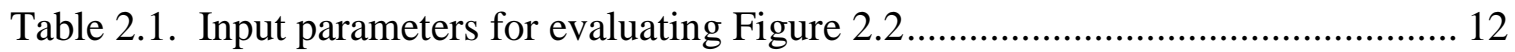

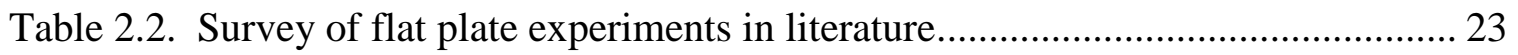

Table 3.1. Available cDAQ measurements............................................................ 39

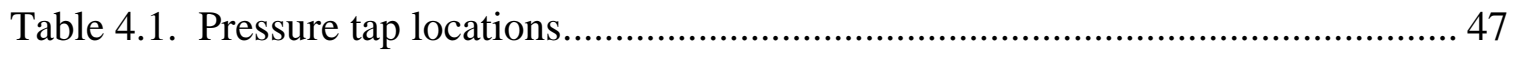

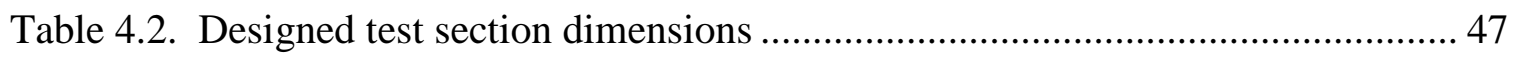

Table 4.3. Laser axial measurement distance from plate trailing edge ......................... 54

Table 4.4. Targeted velocities and flow rates for the initial flow test ......................... 55

Table 5.1. Ideal and measured channel thickness and area...................................... 58

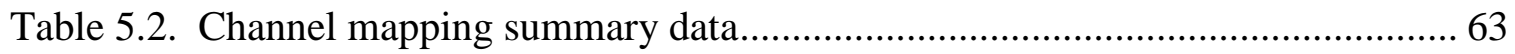

Table 5.3. Measured flow rates and calculated average velocities for free edge

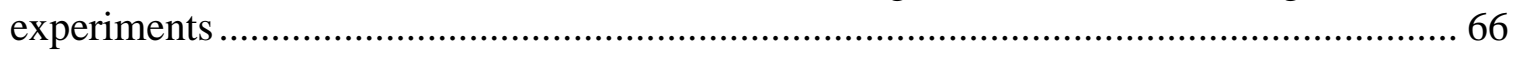

Table 5.4. Lower and upper bounds for snap flow rate during each flow test................ 77

Table 5.5. Measured flow rates and calculated average velocities for pinned edge

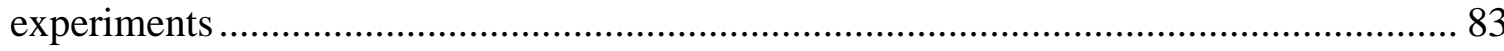

Table 6.1. Miller critical velocity calculation for various channel thicknesses ............... 97

Table 8.1. Analytic pressure drop calculations ....................................................... 109

Table 8.2. Analytic fully developed wall shear stress. ............................................ 109

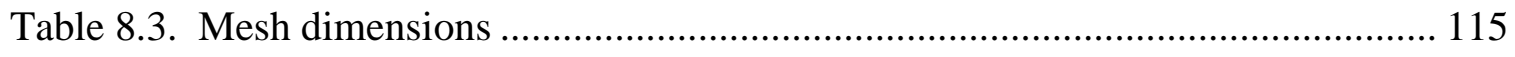

Table 8.4. Analytic near wall y+ values in the fully developed region ...................... 116

Table 8.5. Standard k- $\varepsilon$ pressure drop results .................................................... 117

Table 8.6. Realizable k- $\varepsilon$ pressure drop results .................................................... 118

Table 8.7. Standard (Wilcox) k- $\omega$ pressure drop results........................................ 118 
Table 8.8. SST (Menter) k- $\omega$ pressure drop results ................................................. 119

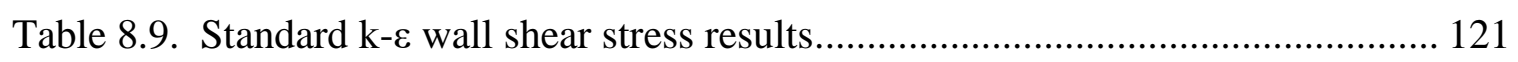

Table 8.10. Realizable k- $\varepsilon$ wall shear stress results ............................................ 122

Table 8.11. Standard (Wilcox) k-w wall shear stress results ................................... 122

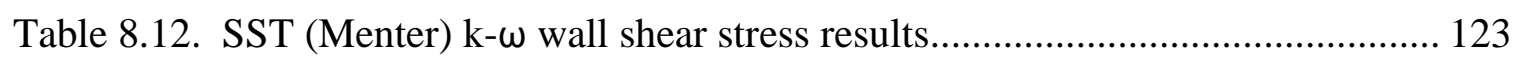

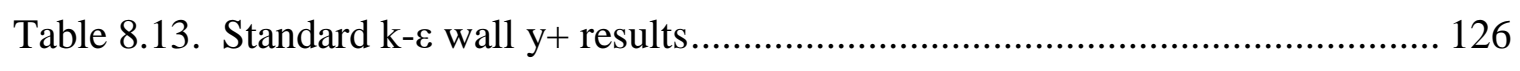

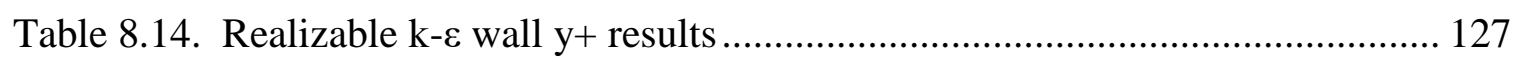

Table 8.15. Standard (Wilcox) k- $\omega$ wall y+ results .............................................. 128

Table 8.16. SST (Menter) k- $\omega$ wall y+ results......................................................... 129

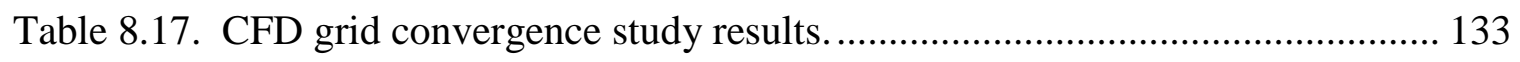

Table 9.1. 6061-T6 FEA plate material properties ................................................. 146

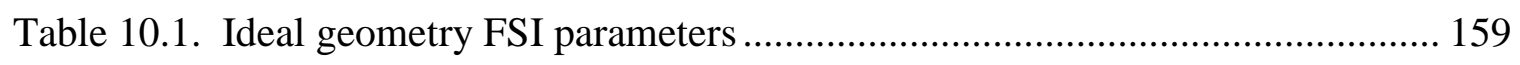

Table 11.1. As-Built geometry FSI parameters ................................................. 169 


\section{NOMENCLATURE}

A Channel cross-sectional area

$a \quad$ Plate thickness

$b \quad$ Wetted plate/channel width

D Flexural rigidity of the plate

$D_{H} \quad$ Hydraulic diameter

E Modulus of elasticity; GCI error

$F_{S} \quad$ Factor of safety

$f \quad$ Colebrook friction factor

$g \quad$ Gravitational acceleration

$h \quad$ Channel thickness

$K \quad$ Pressure loss coefficient

$M_{0} \quad$ Plate edge bending moment

$\dot{m} \quad$ Mass flow rate

$P, p \quad$ Pressure; GCI formal order of accuracy

Re Reynolds Number

$r \quad$ GCI refinement factor

$S \quad$ Tensile reaction force

$s_{j j} \quad$ Strain rate tensor

$t \quad$ Time

$u^{*} \quad$ Friction velocity

$u^{+} \quad$ Dimensionless velocity $\bar{u} \quad$ Average velocity

$u^{\prime}$ Random fluctuation in $\mathrm{X}$ direction

$V, u \quad$ Velocity

$V_{C} \quad$ Miller critical velocity

$v^{\prime} \quad$ Random fluctuation in $\mathrm{y}$ direction

$x \quad$ Spanwise (horizontal) direction across plate/channel width

$w^{\prime}$ Random fluctuation in $\mathrm{z}-$ direction

$x_{i} \quad$ Position

$y \quad$ Axial (vertical) direction along plate/channel length

$y^{+} \quad$ Dimensionless wall distance

$z \quad$ Perpendicular (out-of-plate) direction relative to plate surface

$\Delta z \quad$ Plate deflection

\section{$\underline{\text { Greek Symbols }}$}

$\varepsilon \quad$ Surface roughness; turbulent dissipation per unit mass; GCI grid solution difference

$\mu \quad$ Dynamic viscosity

$v \quad$ Poisson's ratio; kinematic viscosity 


$\begin{array}{ll}\rho & \text { Mass density } \\ \tau & \text { Wall shear stress } \\ \phi & \text { CFD parameter solution } \\ \omega & \begin{array}{l}\text { Turbulent dissipation per unit } \\ \text { time (dissipation rate) }\end{array}\end{array}$

$\underline{\text { Subscripts }}$

$\begin{array}{ll}\text { A } & \text { Channel A } \\ { }_{B} & \text { Channel B } \\ { }_{i, j} & \text { Tensor terms } \\ \text { CON } & \text { Contraction } \\ { }_{\text {EXP }} & \text { Expansion }\end{array}$

$\mathrm{x}$ viii 


\section{ABSTRACT}

As part of the Global Threat Reduction Initiative (GTRI) reactor conversion program, five U.S. High Performance Research Reactors (HPRRs) are currently studying a novel Low Enriched Uranium (LEU) foil based fuel to replace their current High Enriched Uranium (HEU) dispersion fuel. The proposed fuel uses a monolithic U-10Mo foil meat clad in aluminum, whereas the current HEU fuel meat is comprised of Uranium dispersed in an aluminum matrix, before being clad in aluminum. Along with a change in the physical structure of the fuel, the fuel plate thickness has been significantly decreased. Given that these fuel plates are subject to high velocity coolant flow, these changes in the plate design have led to a need to characterize the structural response of the plates in presence of high velocity flow.

The proposed method for completing this analysis is to use novel fluid-structure interaction (FSI) simulations. These simulations are carried out using commercial CFD and FEA solvers Star-CCM+ and Abaqus, and iteratively coupling their solutions together at the interface between the plate and the fluid. Given the unique nature of these simulations, it is necessary to first benchmark and qualify the codes for this analysis.

To generate benchmark quality data, a flow loop and test section have been constructed for studying plate deflection and channel pressure drop under a variety of fluid flow conditions. Experiments have been completed on a single, flat $1.016 \mathrm{~mm}$ (40 mil) thick plate. During flow testing, two leading and trailing edge boundary conditions were considered: fully free and pinned at the midpoint. Results are presented here for flow 
tests at 11 flow rates. Plate deflection was monitored with two laser displacement sensors. The laser sensors were repositioned through seven axial locations and the flow tests repeated, allowing for an understanding of plate deflection behavior along the length of the plate. Additionally, the pressure differential between the two channels is presented at eight axial locations. Similar experimental analysis which considered equally sized fluid channels has been studied by a number of individuals in the past. The work presented here differs however, by intentionally offsetting the plate and creating fluid channels of different thickness. This offset effectively simulates manufacturing tolerances of a real fuel assembly.

FSI simulations based on idealized geometry resulted in significant difference between the experiment and the numeric solution. Therefore, significant effort has been expended to re-evaluate the numeric modeling process. A CFD mesh and turbulence modeling study was completed on a simplified geometry which is characteristic of the unique flow conditions of the experiment. More importantly, a new method for better matching of the numeric model geometry to the experiment geometry is presented. This As-Built model is created through mapping of the experiment fluid channel thicknesses with the laser displacement sensors, and a subsequent morphing of the numeric CFD mesh to match the channel mapping.

These As-Built numeric models have been shown to dramatically improve matching between experiment and numeric solutions, particularly at low- to mid-range flow rates. At higher flow rates, the experiment exhibited a dynamic 'snap' behavior that could not be replicated numerically. Additional interrogation of the boundary conditions revealed a 
possible explanation for this snap, however numeric methods do not yet exist for recreating this behavior. In earlier works which considered equally sized channels, plate deflection was not examined in detail and was found to be largely unpredictable and reliant upon the manufacturing tolerances of the experiment. In the numeric and experimental work presented here, plate deflection behavior at low to mid-range flow rates is qualitatively consistent with theoretical expectations. 


\section{CHAPTER 1: INTRODUCTION}

\section{1: Reactor Core Design}

The University of Missouri Research Reactor (MURR) first went critical in 1966 as a 5 MW research facility. Since an upgrade in 1974, MURR continues to operate today as a $10 \mathrm{MW}$ reactor supporting a wide range of research in life and material science research, and radiopharmaceutical production. In addition to being the United States' most powerful university research reactor, MURR has a proven record as one of the most reliable and capable nuclear research facilities in the world [1]. Like most other research and test reactors constructed during the same time period, MURR utilizes highly enriched uranium (HEU) fuel. Over recent decades, significant progress has been made in converting these reactors to a proliferation resistant low enriched uranium (LEU) fuel. Due to its unique requirements, MURR is one of the few remaining reactors in the United States yet to be converted.

Figure 1.1 shows the MURR core, which consists of 8 concentric fuel elements placed between an inner and an outer pressure vessel. The pressure vessels direct and contain high velocity coolant flow, water, which maintains the fuel plates at safe temperatures while moderating fast neutrons. The core itself is quite compact, with the outer pressure vessel diameter measuring $29.97 \mathrm{~cm}$ (11.80 inches). The inner pressure vessel is 13.51 $\mathrm{cm}$ (5.32 inches) and the fuel plates measure $64.77 \mathrm{~cm}$ (25.5 inches) long. 


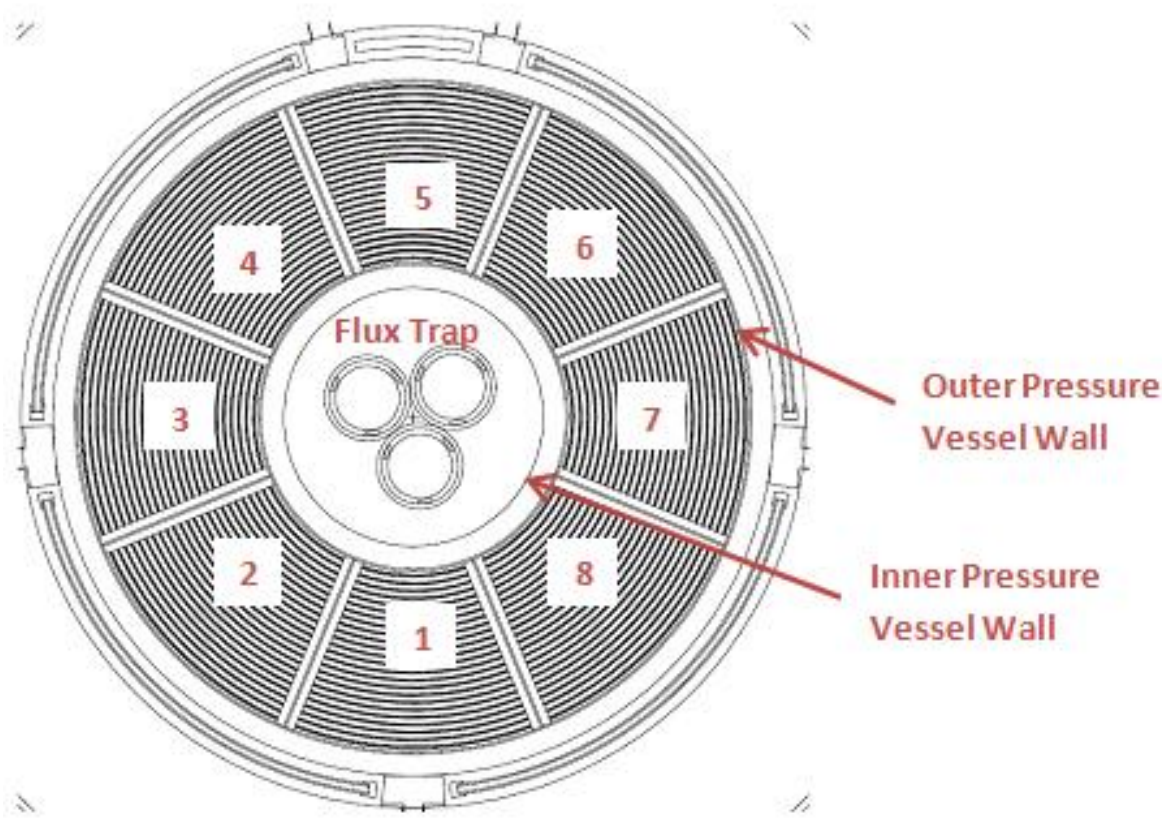

Figure 1.1. Top view of MURR core.

Each HEU fuel element consists of 24 fuel plates, as shown in Figure 1.2. The fuel plates are curved and concentric, providing constant thickness coolant channels between the plates. The plates are held in place through swage joints along the sidewalls and a support comb which is placed radially along both the top and bottom of the fuel plates. Figure 1.3 shows the element includes a handling fixture at both ends to allow for insertion into and removal from the reactor. The plates themselves are nominally 1.27 $\mathrm{mm}(50 \mathrm{mils})$ thick with coolant channels of $2.032 \mathrm{~mm}$ (80 mils) between them. 


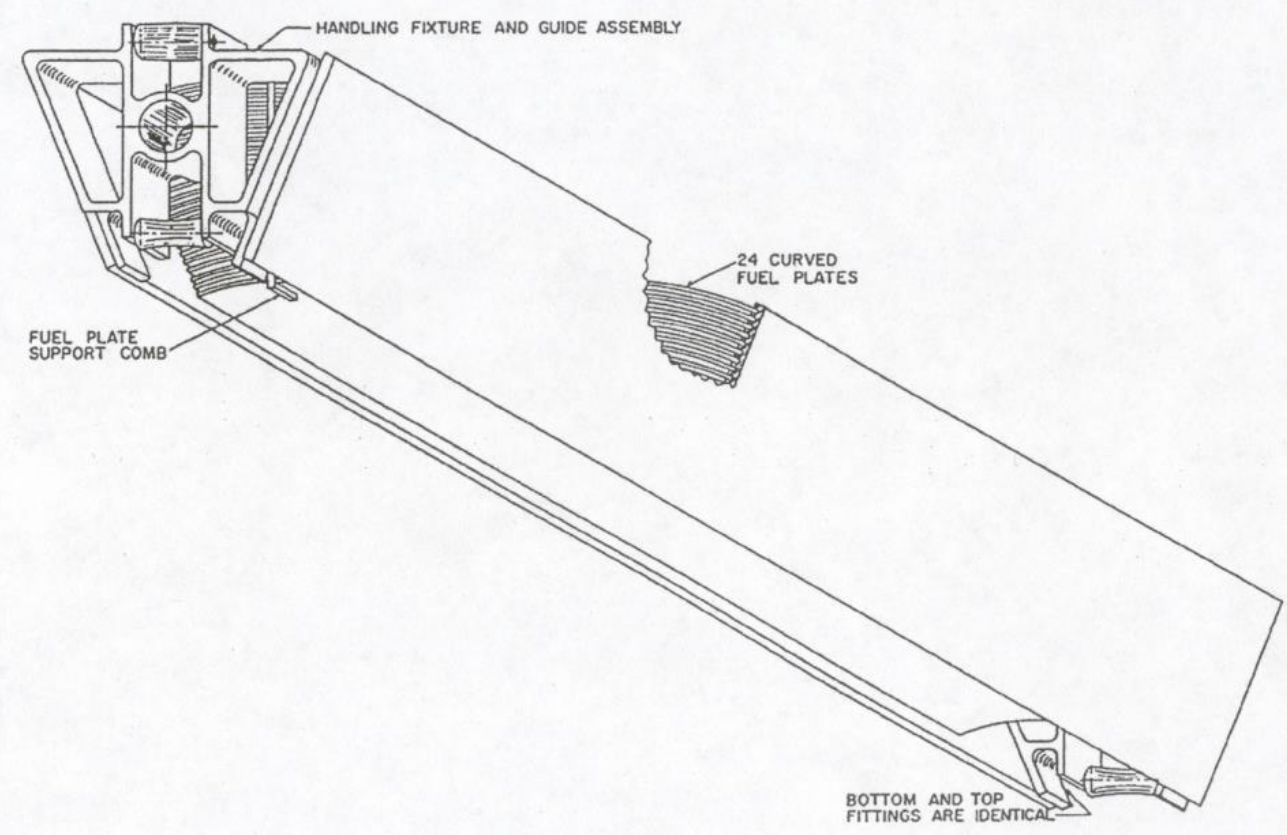

Figure 1.2. MURR fuel element with 24 fuel plates.

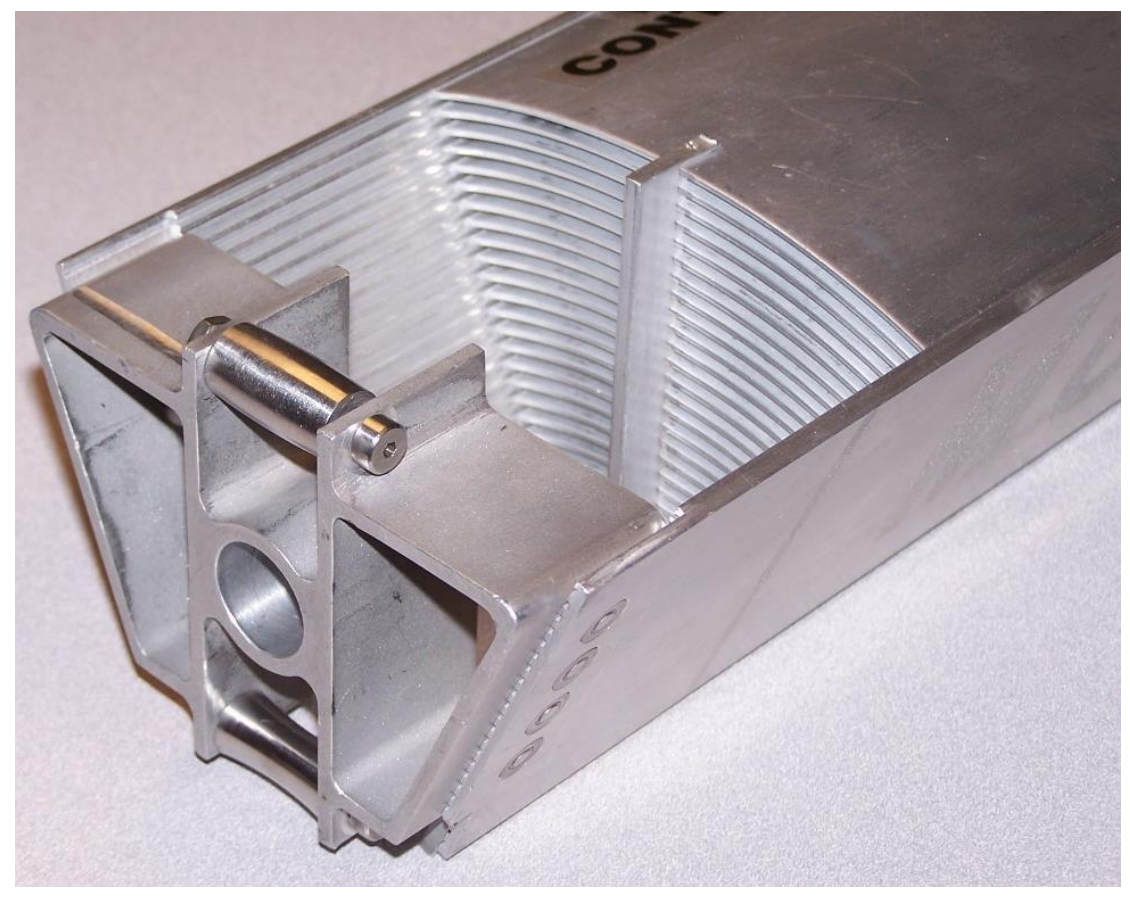

Figure 1.3. MURR fuel element end cap and plates. 


\section{2: Fuel Plate Design}

In 1971 MURR converted from its initial uranium-aluminum alloy fuel to the uraniumaluminide dispersion fuel still used today. This design allowed for an increase in U-235 loading from 650 grams to 775 grams per element at an enrichment of 93\%. The fuel meat portion of the plate is $0.508 \mathrm{~mm}$ ( 20 mils) thick, with $0.381 \mathrm{~mm}$ (15 mils) of aluminum cladding on either side. The current HEU plates are relatively thick at 1.27 $\mathrm{mm}$ (50 mils), curved, and have support combs at the midpoint of the leading and trailing edges. All of these features make the plates fairly stiff and resistant to flow induced deformation. Each HEU fuel element consists of 24 fuel plates [2].

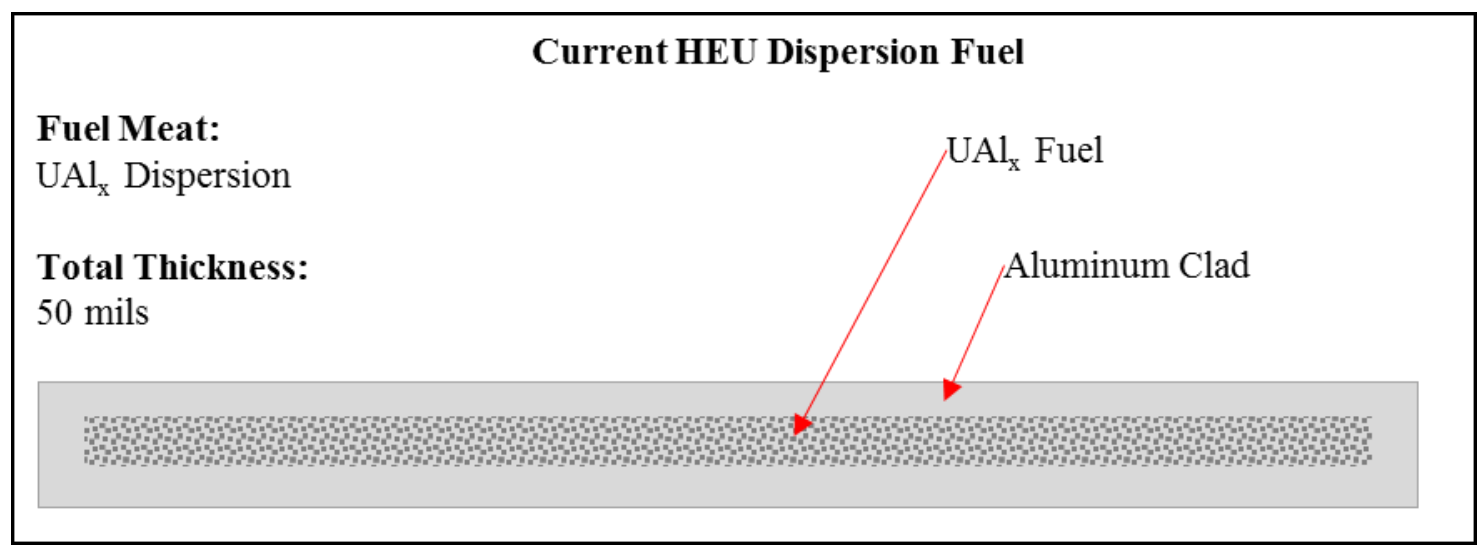

Figure 1.4. Cross section schematic of U-Al dispersion fuel plate.

As part of the effort to convert MURR to an LEU fueled core, two fuel element designs are under consideration. The FSD core maintains the same number of fuel plates, but has them significantly thinner at $0.9652 \mathrm{~mm}$ (38 mils). This allows for $2.3368 \mathrm{~mm}$ (92 mil) thick fluid channels between plates [2]. The alternate CD35 core design uses predominately $1.1176 \mathrm{~mm}$ (44 mil) plates with $2.3368 \mathrm{~mm}$ (92 mil) fluid channels. As a 
result of the extra plate thickness, the number of plates is decreased to 23 [3].

\section{Proposed LEU Monolithic Fuel}

Fuel Meat:

U-10Mo Monolithic

Total Thickness:

38 or 44 mils

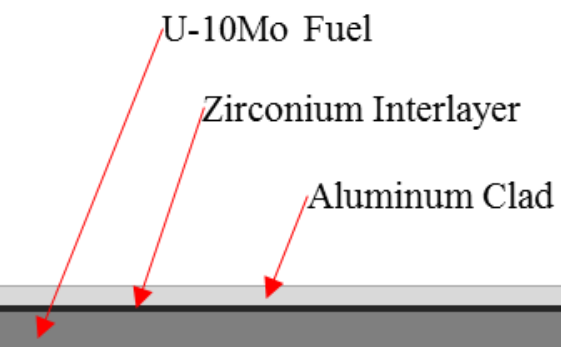

Figure 1.5. Cross section schematic of LEU fuel plate.

\section{3: Motivation and Reactor Conversion}

In order to minimize the risks associated with nuclear materials used in civilian applications, the National Nuclear Security Administration (NNSA) has been working through the Global Threat Reduction Initiative (GTRI) to reduce and secure nuclear materials at sites around the world [4]. As part of the GTRI, significant progress has been made in converting research and test reactors from HEU to LEU through the Reduced Enrichment for Research and Test Reactors (RERTR) program. The remaining reactors in the United States include MURR, the Advanced Test Reactor at Idaho National Laboratory, the High Flux Isotope Reactor at Oak Ridge National Laboratory, the Massachusetts Institute of Technology Reactor, and the National Bureau of Standards Reactor at the National Institute of Standards and Technology. These five High Performance Research Reactors (HPRRs) continue to use HEU fuel due to unique characteristics that have made conversion to LEU difficult. 
In order to reduce the U-235 enrichment from the current $93 \%$ to $19.75 \%$, a dramatic redesign of the fuel has been necessary. With the high U-235 content in the HEU fuel, the fuel meat of a plate is composed of a Uranium-Aluminide dispersion powder. However, in order to reduce the uranium enrichment while simultaneously maintaining an acceptable total loading of U-235, a new fuel design is needed which increases the overall uranium density in the fuel [5]. To that end, a new Uranium-Molybdenum foil is under investigation for use in the HPRRs [6], [7]. For MURR, the proposed fuel plate design uses a U-10Mo fuel meat and an aluminum clad. A thin zirconium layer is deposited between the fuel meat and the clad to prevent the development of fission gas bubbles at the fuel-clad interface. Additionally, the fuel clad thickness has been decreased to allow for additional coolant flow between the fuel plates. This additional coolant volume in the core provides greater neutron moderation, resulting in longer life and lower cost for the fuel elements. With the layered structure of the fuel, as well as the overall thinning of the fuel plates, the plates may be more susceptible to flow induced deflection. This largely depends on the material properties of the fuel components and water flow velocity in neighboring channels. A review of the literature reveals a wide range of material property values, largely depending on the heat treatment and manufacturing processes. Table 1.1 provides a glimpse of the most relevant structural property values for the three layers in an LEU fuel plate. 
Table 1.1. Selected material properties for LEU fuel components

\begin{tabular}{cccc} 
Material & $\begin{array}{c}\text { 0.2 \% Offset Yield } \\
\text { Strength (MPa) }\end{array}$ & $\begin{array}{c}\text { Elastic Modulus } \\
\text { (GPa) }\end{array}$ & Poisson's Ratio \\
\hline 6061-T6 Al & $290[8]$ & $68[8]$ & 0.33 \\
U-10Mo & $940[6]$ & $87[9]$ & $0.35[9]$ \\
Zr & $90-110[10]$ & $86-98[10]$ & 0.34
\end{tabular}

This work focuses on development of experiments and numeric models to aid in analysis of fluid-structure interaction of the proposed LEU fuel. The experiments are meant to provide data for validation of the numeric models. The numeric models involve using new coupling and stabilization methodologies for simultaneous CFD and FEA analyses.

\section{4: Fluid Structure Interaction (FSI)}

In nuclear applications, high velocity water flow is required to cool fuel plates. This design results in a complex mechanical interaction between the fluid and the fuel plate. In order for a fuel design to be qualified for use in a reactor, the potential for flowinduced deflection of the fuel plates must be assessed. Complex, expensive experiments have been used in the past as the primary method for assessing the fluid structure interaction. While extremely helpful in reactor design, these experiments were limited by test section design, flow rate, and instrumentation. Experiments are also difficult to modify, thereby limiting the ability to conduct 'what-if' types of analyses.

In order to overcome these limitations in validating the current fuel designs, a two-step approach using new experiments and numeric modeling is necessary. The new flow experiments use modern instrumentation and allow for direct visual observation of a plate 
during flow. Using the data gained from these experiments, validation of numeric models is possible. These models will allow for more detailed analysis than the experiments.

For analyzing fluid flow and heat transfer, computational fluid dynamics (CFD) codes such as Star-CCM+ are used. Structures are analyzed with finite element analysis (FEA) codes such as Abaqus. Historically, these codes were used independent of each other, providing a decent but incomplete understanding of reactor fluid structure interaction. With continued advances in computational capacity, it has recently become feasible to couple together CFD and FEA codes for integrated FSI analysis. Before this new capability can be used for reactor fuel analysis however, it must first be validated and benchmarked against experiment data.

The ultimate purpose of this study is to interrogate and validate methods for fluidstructure interaction simulation of reactor fuel assemblies. Additionally, experiments utilizing new metrology techniques will be used for generation of new benchmark data. The geometry used for this analysis included an intentionally offset plate allowing for evaluation of the effect of non-uniform fluid channels, and an understanding of the sensitivity to manufacturing tolerances. 


\section{CHAPTER 2: BACKGROUND \& LITERATURE REVIEW}

\section{1: Analytic Models}

\subsection{1: Critical Flow Velocity}

For more than half a century, scientists have investigated various aspects of plate stability under high velocity fluid flow. In 1958, Miller developed an analytic method for estimating the critical flow velocity that could cause the collapse of fluid sub-channels in a plate arrays. For stacks on the order of 10 plates, Eq. 2.1 is Miller's critical velocity [11].

$$
V_{C}=\left[\frac{15 E a^{3} h}{\rho b^{4}\left(1-v^{2}\right)}\right]^{\frac{1}{2}}
$$

For the case of a single plate placed in the middle of a rectangular duct, Miller

determined that the critical velocity of Eq. 2.1 is increased by a factor of $\sqrt{2}$. This results from the presence of rigid channel walls instead of other moving plates seen in a stack. Including this factor gives the Miller critical velocity for a single plate assembly as Eq. 2.2 .

$$
V_{C}=\left[\frac{30 E a^{3} h}{\rho b^{4}\left(1-v^{2}\right)}\right]^{\frac{1}{2}}
$$

Miller's critical velocity provides the fluid velocity at which the deflected plate collapses due to the pressure force exerted by the fluid. While this model provides a quick and simple means of estimating the velocity for plate instability, it does not allow for differing thicknesses of neighboring channels. As a result, Eqns. 2.1 and 2.2 also fail to 
account for the flow redistribution that would result from differing initial channel thicknesses or plate deflection above the critical velocity.

Concerning the plate itself, Miller assumes a homogenous, single material plate.

Obviously, for a real fuel plate this assumption is not entirely accurate. To address this deficiency, analytic modeling has been completed by Jensen and Marcum to integrate the rigidity of a multilayer structure into Miller's theory for both flat and curved plates. This method involves rewriting Miller's equations in terms of the plate's flexural rigidity, and then evaluating the flexural rigidity for a multi-layer structure [12] [13].

\subsection{2: Beam \& Plate Bending}

Figure 2.1 shows the generic case of a beam or plate with opposite fixed edges under a uniform pressure, $P$. The plate deflection, $\Delta z$, varies as a function of the horizontal location, $x$, across the full width, $b$. As the plate deflects, reaction forces, $S$, are generated, putting the plate in tension. From the clamping condition along the edges, moments $M_{0}$ are generated.

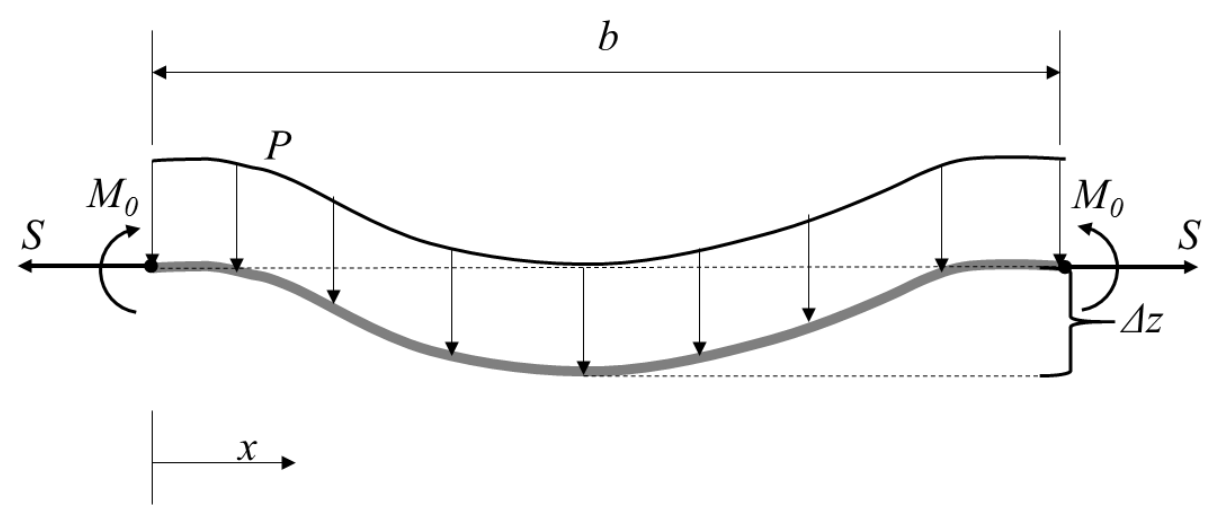

Figure 2.1. Deflection of plate (long beam) under uniform load with two opposite fixed edges 
The plate's resistance to bending is defined by the flexural rigidity, $D$, as defined in equation $2.3[14]$.

$$
D=\frac{E a^{3}}{12\left(1-v^{2}\right)}
$$

If the deflection is small when compared to the plate thickness, $a$, then the membrane stresses (and reaction forces, $S$ ), are insignificant and can typically be neglected. This results in the simple beam deflection calculation of equation 2.4 [15]. For the maximum deflection at $x=b / 2$, equation 2.5 can be utilized.

$$
\begin{gathered}
\Delta z=\frac{P x^{2}}{24 D}(b-x)^{2} \\
\Delta z_{\text {max }}=\frac{P b^{4}}{384 D}
\end{gathered}
$$

If, however, the plate deflection is of the same order of magnitude as the plate thickness, then the tensile reaction forces, $S$, at the ends will be significant. To account for these reactions, Timoshenko proposed equation 2.6. Note that the parameter $\mathrm{u}$ is solved iteratively with equation 2.7 .

$$
\begin{aligned}
& \Delta z=\frac{P b^{4}}{16 u^{3} D \tanh u}\left\{\frac{\cosh \left[u\left(1-\frac{2 x}{b}\right)\right]}{\cosh u}-1\right\}+\frac{P b^{2}(b-x) x}{8 u^{2} D} \\
& \frac{E^{2} a^{8}}{\left(1-v^{2}\right)^{2} P^{2} b^{8}}=-\frac{81}{16 u^{7} \tanh u}-\frac{27}{16 u^{6} \sinh ^{2} u}+\frac{27}{4 u^{8}}+\frac{9}{8 u^{6}}
\end{aligned}
$$

As with the simple beam equation, the maximum deflection at the midpoint can be solved by setting $x=b / 2$, as in equations 2.8 and 2.9 . 


$$
\begin{gathered}
\Delta z_{\max }=\frac{P b^{4}}{384 D} f_{1}(u) \\
f_{1}(u)=\frac{24}{u^{4}}\left(\frac{u^{2}}{2}+\frac{u}{\sinh u}-\frac{u}{\tanh u}\right)
\end{gathered}
$$

A simple python script for solving both the simple beam and Timoshenko plate equations is included as Appendix A. Using the values of Table 2.1, these equations were used to create the plot of Figure 2.2. From this plot, it is clear that both equations yield similar results at lower pressures. However, as the deflection approaches the order of magnitude of the plate thickness and the membrane stresses increase, the simple beam equation increasingly over-predicts deflection when compared to the plate equation.

Table 2.1. Input parameters for evaluating Figure 2.2

\begin{tabular}{cc}
\hline Parameter & Value \\
\hline$a$ & $1.016 \mathrm{~mm}$ \\
$b$ & $110.2868 \mathrm{~mm}$ \\
$x$ & $\mathrm{~b} / 2=55.1434 \mathrm{~mm}$ \\
$E$ & $68.9 \mathrm{GPa}$ \\
$v$ & 0.33 \\
\hline
\end{tabular}




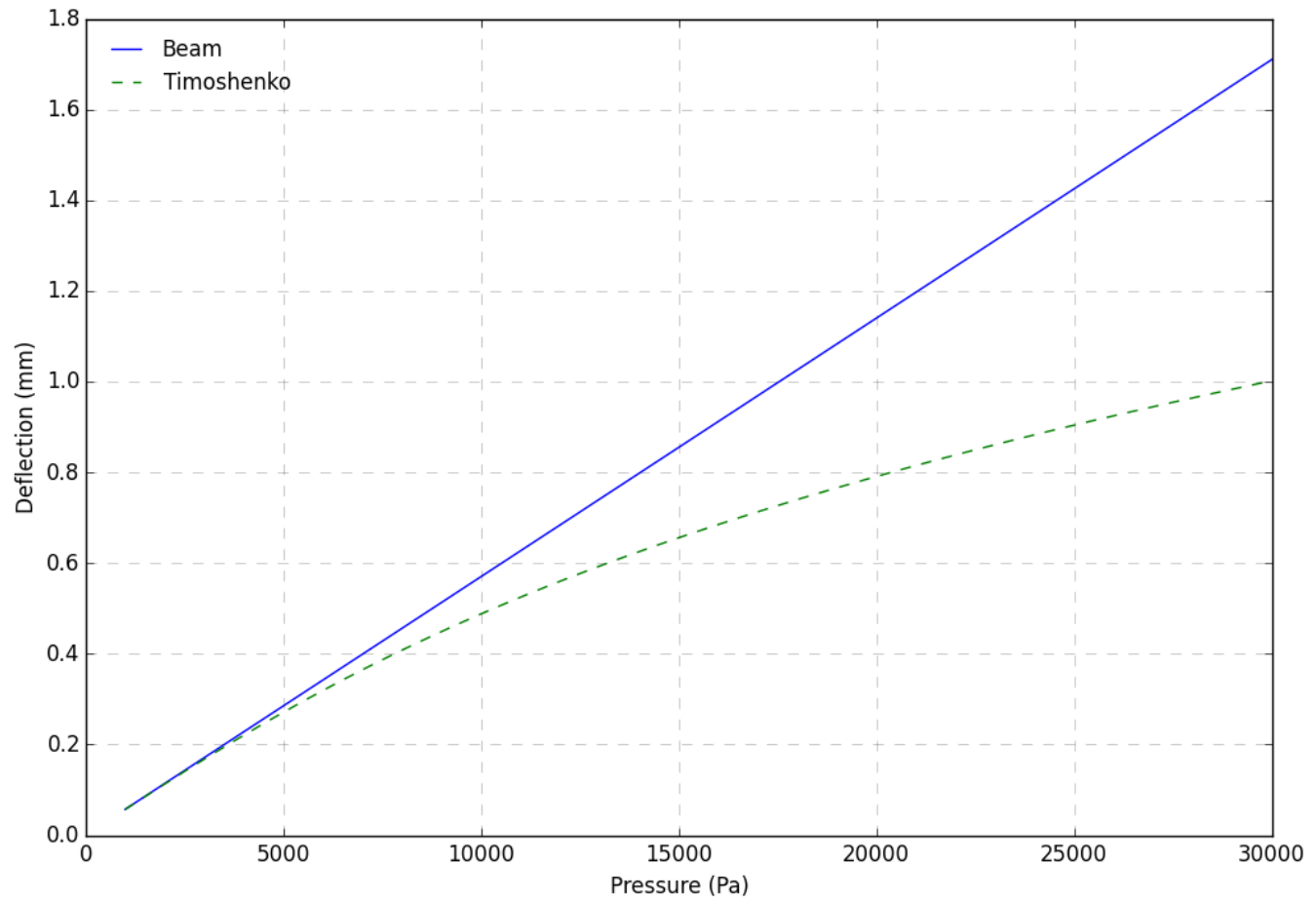

Figure 2.2. Simple beam deflection vs. Timoshenko plate deflection

Thus far, the beam bending models have focused solely on plates of a single material. However, from Figure 1.5 it is clear that the actual reactor fuel plates are composed of multiple layers. To determine the deflection of a multi-layered plate, the elastic modulus, $E$, can be modified while keeping all other parameters the same. For the case of a three layer beam, where the two outer layers are of the same material and thickness, an effective Young's modulus for a beam with the same dimensions, made of a homogenous material, can be determined. Using the cross-section of Figure 2.3, equation 2.10 can be used to calculate the equivalent modulus $\mathrm{E}_{\mathrm{C}}$. 


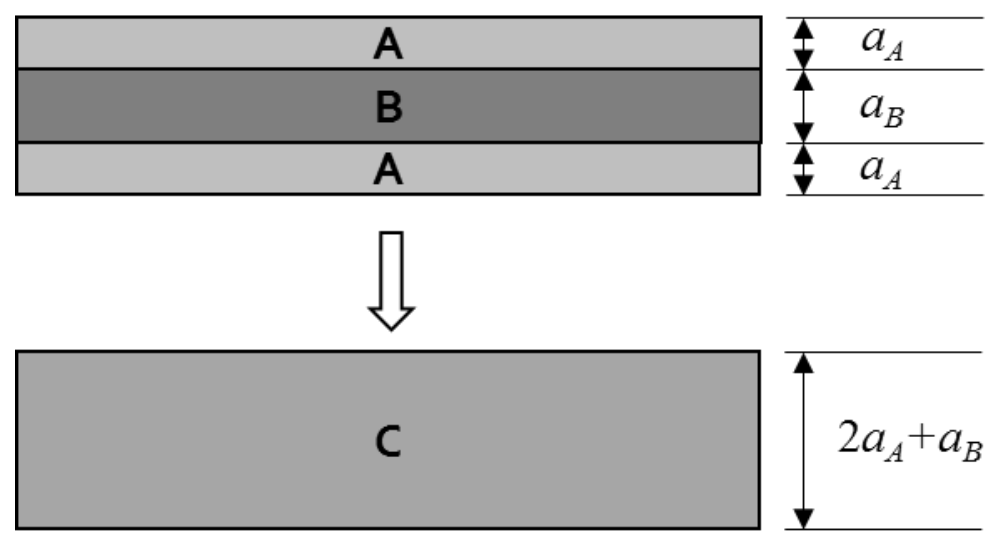

Figure 2.3. Conversion of a composite plate if Young's Moduli $E_{A}$ and $E_{B}$ to a homogenous plate of Young's Modulus $E_{\mathbf{C}}$

$$
E_{C}=E_{A} \frac{2 a_{A}^{3}+6 a_{A}\left(a_{A}+a_{B}\right)^{2}+\frac{E_{B}}{E_{A}} a_{B}^{3}}{\left(2 a_{A}+a_{B}\right)^{3}}
$$

As an example to approximate an actual MURR CD35 LEU fuel plate, material A can be 6061-T6 aluminum with a thickness $a_{A}=0.3048 \mathrm{~mm}$, and material B can be U-10Mo with a thickness $a_{B}=0.5080 \mathrm{~mm}$ [5]. The elastic moduli are $\mathrm{E}_{\mathrm{A}}=68 \mathrm{GPa}$ and $\mathrm{E}_{\mathrm{B}}=87$ GPa from Table 1.1. Using equation 2.10, $\mathrm{E}_{\mathrm{C}}=69.8 \mathrm{GPa}$. Despite the center layer being thicker, the modulus of the outer layers dominates. This is due to the outer layers being some distance away from the neutral axis of the beam and therefore having a larger resistance to bending.

\subsection{3: Fluid Flow}

For the generic case of axial fluid flow around a plate, there are several factors contributing to the pressure drop through the test section, and therefore to the pressure that causes plate deflection. Along the length of the flow, anytime the channel crosssectional area changes there is a recoverable Bernoulli pressure drop. Using Figure 2.4 
for reference, with subscripts $A$ and $B$ representing the upstream and downstream locations, respectively, equation 2.11 describes the Bernoulli component of pressure drop.

$$
\begin{aligned}
P_{B}-P_{A}=\frac{1}{2} \rho\left(V_{A}^{2}-V_{B}^{2}\right)+\rho g\left(y_{A}-y_{B}\right) \\
=\frac{1}{2 \rho b^{2}}\left(\frac{\dot{m}_{A}^{2}}{h_{A}^{2}}-\frac{\dot{m}_{B}^{2}}{h_{B}^{2}}\right)+\rho g\left(y_{A}-y_{B}\right)
\end{aligned}
$$

As the flow approaches the leading of the plate and splits abruptly into the channels, there is an irrecoverable pressure loss due to the sudden contraction. At present, there is an extremely limited number of geometries in available literature for determining this pressure drop component. The available empirical correlations tend to focus on one of two cases:

1) Flow from a single large channel into a single smaller channel

2) Flow from a single large channel into many equally size smaller channels 


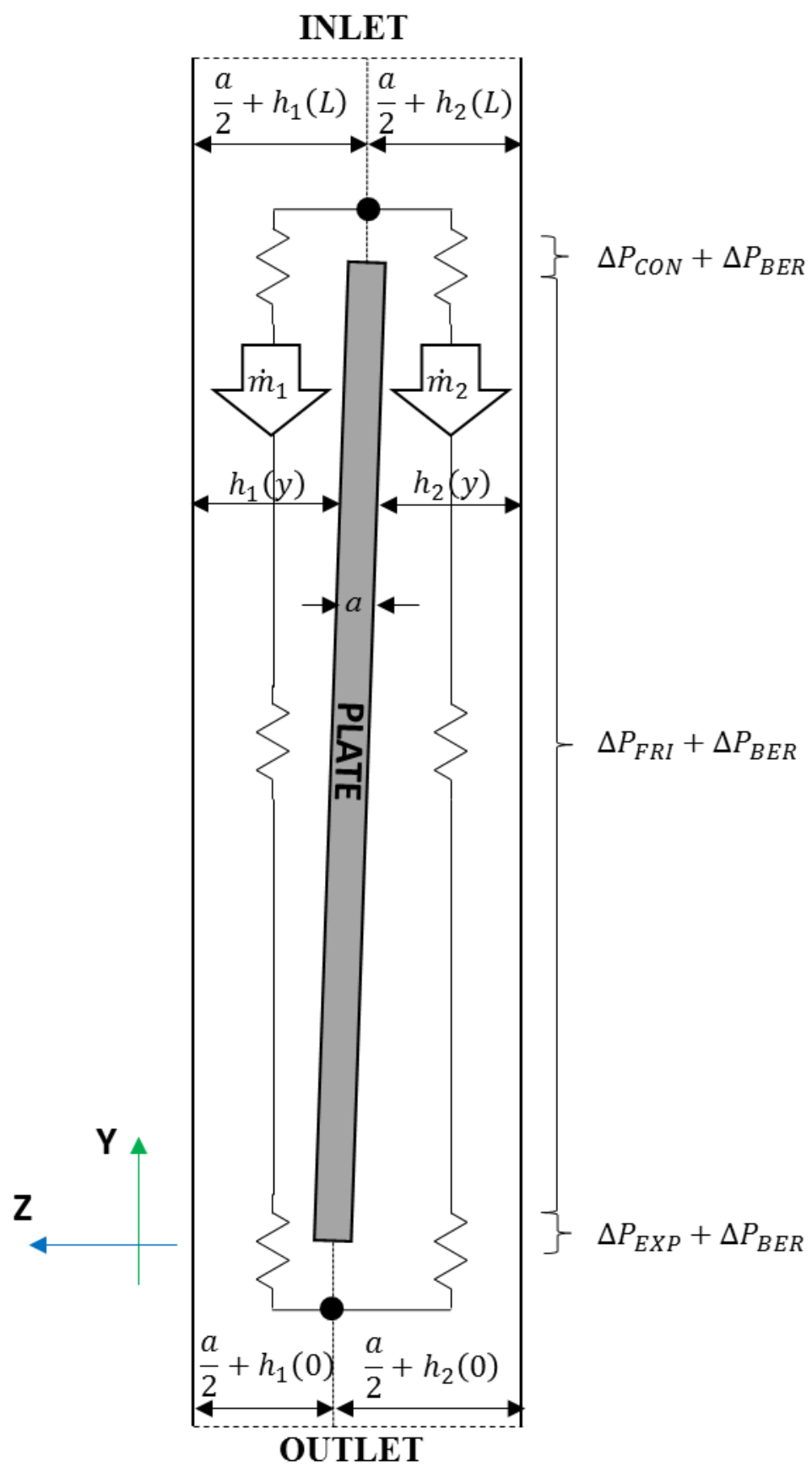

Figure 2.4. Axial fluid resistance diagram around a deflected plate

The geometry of interest in this case fails to fit either of these models well, with flow going from a single large channel into two smaller channels of differing thicknesses. As an approximation, the correlations from Blevins for the first case are provided [16]. 
Equation 2.12 is the theoretical contraction $K$ loss coefficient, and equation 2.13 is an alternative empirical coefficient. To solve for the contraction pressure loss, the velocity in the sub-channel is used.

$$
\begin{gathered}
K_{\text {CON }}=\frac{1}{2}\left(1-\frac{b h(L)}{b\left(\frac{a}{2}+h(L)\right)}\right) \\
K_{\text {CON }}=0.5781+0.3954\left(\frac{b h(L)}{b\left(\frac{a}{2}+h(L)\right)}\right)^{0.5}-4.5385\left(\frac{b h(L)}{b\left(\frac{a}{2}+h(L)\right)}\right) \\
+14.24\left(\frac{b h(L)}{b\left(\frac{a}{2}+h(L)\right)}\right)^{\frac{3}{2}}-19.22\left(\frac{b h(L)}{b\left(\frac{a}{2}+h(L)\right)}\right)^{2} \\
+8.540\left(\frac{b h(L)}{b\left(\frac{a}{2}+h(L)\right)}\right)^{\frac{5}{2}}
\end{gathered}
$$

Much like the contraction coefficients, there are limited models available for sudden flow expansion at the trailing edge of the plate. Equation 2.14 represents the expansion pressure loss from a single sub-channel into the outlet plenum. As before, to solve for the resulting expansion pressure loss the velocity in the smaller channel is used.

$$
K_{E X P}=\left(1-\frac{b h(0)}{b\left(\frac{a}{2}+h(0)\right)}\right)^{2}
$$


The final and most significant component of pressure drop is the irrecoverable frictional loss as turbulent flow moves through the channels. To solve for the friction pressure drop, the friction factor, $f$, is solved using the Colebrook equation (eq. 2.17) [17] [18].

$$
\begin{gathered}
D_{H}=\frac{2 b h(y)}{b+h(y)} \\
R e=\frac{\rho V D_{H}}{\mu}=\frac{\dot{m} D_{H}}{\mu b h(y)} \\
\frac{1}{\sqrt{f}}=-2 \log _{10}\left(\frac{\varepsilon}{3.7 D_{H}}+\frac{2.51}{\operatorname{Re} \sqrt{f}}\right) \\
K_{F R I}=f \frac{(\Delta y)}{D_{H}}
\end{gathered}
$$

Therefore, for flow through a channel, the irrecoverable pressure losses are described by equation 2.19 .

$$
\begin{aligned}
\Delta P_{K}=\frac{\rho V^{2}}{2}\left(K_{C O N}+K_{F R I}+K_{E X P}\right) & \\
& =\frac{\dot{m}^{2}}{2 \rho b^{2} h^{2}}\left(K_{C O N}+K_{F R I}+K_{E X P}\right)
\end{aligned}
$$

The recoverable pressure losses result from the Bernoulli pressure change at the plate leading and trailing edges, as well as the gradual changes in channel area along the length of the flow. To determine the flow rate through both channels, the total pressure drops (recoverable + irrecoverable) through each channel are set equal to each other and the flow rates are adjusted. This causes a net pressure force on the plate, resulting in plate deflection, and further flow redistribution. 
In addition to pressure drop, analytic solutions for the wall shear stress are useful for numeric model benchmarking. Equation 2.20 is the wall shear stress calculation. This equation will be used later in determining the validity of a CFD finite volume discretization.

$$
\tau_{0}=\frac{\Delta P}{L} \frac{D_{H}}{4}=\frac{1}{8} f \rho \bar{u}^{2}
$$

Finally, in CFD simulations, the accuracy of the solution is closely tied to the wall shear stress, and the rate at which the velocity profile develops from the wall outward. This is measured through the non-dimensional wall y+ calculation of equation 2.23 [19].

$$
\begin{gathered}
u^{*}=\sqrt{\frac{\tau_{0}}{\rho}} \\
u^{+}=\frac{u}{u^{*}} \\
y^{+}=\frac{y u^{*}}{v} \\
y=\frac{y^{+} \mu}{\rho \bar{u} \sqrt{\frac{f}{8}}}
\end{gathered}
$$

\section{2: Experiments}

\subsection{1: Flat Plate Experiments}

In an effort to validate Miller's equations for critical velocity, Zabriskie completed a series of single and multi-plate experiments in 1958 [20]. Zabriskie also tested the impact of a comb on the critical velocity. Those experiments attempted to maintain the assumptions of Miller's theory - notably that the channels were all of equal size and that 
the mass flow rate into each channel was the same. In some of the flat plate experiments, it was observed that a slight bowing of 6 mils in the plate caused a dramatic change in the plate's deflection behavior. Zabriskie observed that channel variations of only a few mils from the ideal were adequate to cause significant changes in the observed critical velocity. Zabriskie also noted that the deflections tended to start near the leading edge of the plate, where the pressure differential was the greatest, and progress down the plate as flow increased. Finally, an unusual result was noted in tests of a $0.8123 \mathrm{~mm}$ (32 mil) plate, where the plate was observed to begin deflecting in one direction, before suddenly switching to deflection in the opposite direction.

Additional experiments by Zabriskie in 1959 sought to analyze the impact of larger length to width ratios [21]. He noted that it was impossible to build an experiment with equal flow resistances through both channels, and that this would be enough to bias the plate's deflection in one direction. The plate was observed to only deflect in one direction at a time, and no large scale waves or oscillations were noticed. Water absorption of the plexi-glass plates used in those experiments affected many of the results. An experiment conducted after the flow tests showed that water absorption by the plexi-glass could, over time, lead to significant deformation.

In 1962, Groninger conducted experiments with multi-plate assemblies up to 1.9 times the Miller critical velocity [22]. These experiments used strain gage monofilament sensors embedded into the side edges of the plates to determine deflection. However, the results were limited due to issues surrounding thermal expansion around the gage connectors and water leakage into the gages. His investigation concluded that the onset 
of plate deflection is largely the result of existing perturbations in the plate geometry. Additionally, he found that while a support comb on the inlet did help to reduce overall deflection magnitude, there were still significant deflections 2 to 3 plate widths from the inlet. In some tests without a comb, the maximum deflection occurred 300 to $380 \mathrm{~mm}$ (12 to 15 inches) from the leading edge of the plate. Finally, Groninger noted that above 1.9 times the Miller critical velocity, a high amplitude, high frequency vibration might occur.

In an effort to address the lack of static deflection experimental data, Smissaert conducted flow experiments on arrays of five plates using strain gages to determine the plate deflection [23]. Although he used a different type of gage than Groninger, Smissaert also embedded the gages into the side edges of the plates. Additionally, by using 10 gages along the length of a plate, he sought to analyze the profile of plate deflection under flow. These experiments noted that the leading edge condition was the primary driver in deflection. Additionally, the plates were noted to deform in a wave pattern, with the wavelength tied to the flow velocity. Like Groninger, Smissaert noted significant plate flutter at velocities well above the theoretical Miller critical velocity.

In designing the Advanced Neutron Source reactor, Swinson conducted flow experiments on involute plates which were planned for that reactor [24] [25] [26]. Similar plates are used in other reactors, such as HFIR, and have a unique design that welds curved, involute plates between inner and outer cylinders. Since the plate design is inherently stiff, the experiments used epoxy plates with a lower elastic modulus so that less flow 
would be required to cause deflection. The results from the experiment are used in validation of an analytic model.

As part of an effort to evaluate the fuel design of the Replacement Research Reactor (RRR) at the Austrailian Science and Technology Organisation (ANSTO), Ho conducted experiments on assemblies with two plates [27]. These flow tests drove the plates to plastic deformation. The plate response was monitored through a series of strain gages and pressure taps, and the clear plexi-glass design of the test section allowed for direct visual observation.

More recently, Liu et al. have examined plate vibration with laser displacement sensors placed at the center-point of a test plate [28] [29]. These experiments differed from previous experiments by holding the plate fixed at its four corners. The side edges were allowed to move freely in and out of the plane of the plate. This boundary condition hugely magnified plate deflection. The laser measurements in these experiments took the absolute distance from the laser to the plate. This measurement technique, combined with the laser sensor being only semi-fixed to the test section, makes the resulting sensor data highly susceptible to motion and vibration of the apparatus and not necessarily the plate.

With the wide array of flat plate experiments in literature, Table 2.2 is helpful in comparing experiments from different sources. 
Table 2.2. Survey of flat plate experiments in literature

\begin{tabular}{|c|c|c|c|c|c|c|c|c|c|c|}
\hline Source & Instruments & $\begin{array}{c}\text { Plate } \\
\text { Material }\end{array}$ & $\begin{array}{l}\text { Number } \\
\text { of Plates }\end{array}$ & $\begin{array}{c}\text { Plate } \\
\text { Width }\end{array}$ & $\begin{array}{c}\text { Plate } \\
\text { Thickness }\end{array}$ & $\begin{array}{c}\text { Plate } \\
\text { Length }\end{array}$ & $\begin{array}{c}\text { Channel } \\
\text { Thickness }\end{array}$ & Comb & $\begin{array}{c}\text { Calculated } \\
\text { Miller } \\
\text { Velocity }\end{array}$ & $\begin{array}{c}\text { Observed } \\
\text { Critical } \\
\text { Velocity }\end{array}$ \\
\hline & & & & $\begin{array}{c}b \\
(\mathbf{m m})\end{array}$ & $\begin{array}{c}a \\
(\mathbf{m m})\end{array}$ & $\begin{array}{c}L \\
(\mathbf{m m})\end{array}$ & $\begin{array}{c}h \\
(\mathbf{m m})\end{array}$ & & $\begin{array}{c}V_{C} \\
(\mathbf{m} / \mathbf{s})\end{array}$ & $(\mathrm{m} / \mathrm{s})$ \\
\hline \multirow{7}{*}{$\begin{array}{c}\text { Zabriskie } \\
\text { (1958) } \\
{[20]}\end{array}$} & \multirow{7}{*}{$\begin{array}{c}\text { impact } \\
\text { probes; static } \\
\text { pressure; } \\
\text { visual }\end{array}$} & \multirow{3}{*}{ Aluminum } & \multirow{3}{*}{1} & \multirow{3}{*}{101.6} & 0.5080 & 508 & $\begin{array}{l}3.1750 \\
1.5875\end{array}$ & No & $\begin{array}{l}3.05 \\
4.42\end{array}$ & $\begin{array}{l}2.26-4.39 \\
4.57-7.01\end{array}$ \\
\hline & & & & & 0.8128 & 508 & $\begin{array}{l}3.1750 \\
1.5875\end{array}$ & & $\begin{array}{c}6.25 \\
11.92\end{array}$ & $\begin{array}{c}5.64-6.64 \\
9.91\end{array}$ \\
\hline & & & & & 1.5875 & 508 & $\begin{array}{l}3.1750 \\
1.5875\end{array}$ & & $\begin{array}{l}16.84 \\
2.59\end{array}$ & $\begin{array}{l}12.8 \\
1.68\end{array}$ \\
\hline & & Plexi & 1 & $\begin{array}{c}101.6 \\
76.2\end{array}$ & 1.5875 & 508 & 3.1750 & No & $\begin{array}{l}3.75 \\
6.64\end{array}$ & $\begin{array}{c}3.41-4.11 \\
5.49\end{array}$ \\
\hline & & Plexi & 8 & 101.6 & 1.5875 & 508 & 3.175 & $\begin{array}{l}\text { No } \\
\text { Yes }\end{array}$ & $\begin{array}{c}2.66 \\
---\end{array}$ & $\begin{array}{c}2.50-2.99 \\
>6.40\end{array}$ \\
\hline & & \multirow[b]{2}{*}{ Aluminum } & \multirow{2}{*}{9} & \multirow[b]{2}{*}{101.6} & \multirow[b]{2}{*}{0.8128} & \multirow[b]{2}{*}{508} & \multirow[b]{2}{*}{3.175} & No & 4.36 & 3.96 \\
\hline & & & & & & & & $\begin{array}{l}\text { Yes } \\
\text { No } \\
\text { Yes }\end{array}$ & $\begin{array}{c}--- \\
4.36 \\
---\end{array}$ & $\begin{array}{l}>5.64 \\
3.11 \\
>6.40\end{array}$ \\
\hline
\end{tabular}

continued on next page... 


\begin{tabular}{|c|c|c|c|c|c|c|c|c|c|c|}
\hline Source & Instruments & $\begin{array}{c}\text { Plate } \\
\text { Material }\end{array}$ & $\begin{array}{l}\text { Number } \\
\text { of Plates }\end{array}$ & $\begin{array}{c}\text { Plate } \\
\text { Width }\end{array}$ & $\begin{array}{c}\text { Plate } \\
\text { Thickness }\end{array}$ & $\begin{array}{c}\text { Plate } \\
\text { Length }\end{array}$ & $\begin{array}{l}\text { Channel } \\
\text { Thickness }\end{array}$ & Comb & $\begin{array}{c}\text { Calculated } \\
\text { Miller } \\
\text { Velocity }\end{array}$ & $\begin{array}{c}\text { Observed } \\
\text { Critical } \\
\text { Velocity }\end{array}$ \\
\hline & & & & $\begin{array}{c}b \\
(\mathbf{m m})\end{array}$ & $\begin{array}{c}a \\
(\mathbf{m m})\end{array}$ & $\begin{array}{c}L \\
(\mathbf{m m})\end{array}$ & $\begin{array}{c}\boldsymbol{h} \\
(\mathbf{m m})\end{array}$ & & $\begin{array}{c}V_{C} \\
(\mathbf{m} / \mathbf{s})\end{array}$ & $(\mathbf{m} / \mathbf{s})$ \\
\hline \multirow{8}{*}{$\begin{array}{c}\text { Zabriskie } \\
(1959) \\
{[21]}\end{array}$} & \multirow{8}{*}{$\begin{array}{l}\text { impact } \\
\text { probes; static } \\
\text { pressure; } \\
\text { visual }\end{array}$} & \multirow{6}{*}{ Plexi } & \multirow{6}{*}{1} & \multirow{6}{*}{101.6} & 1.2700 & $\begin{array}{c}508 \\
1016 \\
1448\end{array}$ & 2.5400 & Yes & 2.40 & \\
\hline & & & & & 1.0160 & $\begin{array}{c}508 \\
1016 \\
1448\end{array}$ & 2.5400 & Yes & 1.72 & \\
\hline & & & & & 1.0160 & $\begin{array}{c}508 \\
1016 \\
1448\end{array}$ & 4.0640 & Yes & 2.17 & \\
\hline & & & & & 1.5240 & $\begin{array}{c}508 \\
1016\end{array}$ & $\begin{array}{l}1.5875 \\
2.5400\end{array}$ & Yes & $\begin{array}{l}2.50 \\
8.74\end{array}$ & \\
\hline & & & & & 1.2700 & $\begin{array}{l}1016 \\
1448\end{array}$ & 2.5400 & Yes & 2.40 & \\
\hline & & & & & 1.5240 & $\begin{array}{c}508 \\
1016 \\
1448\end{array}$ & 2.5400 & Yes & 3.26 & \\
\hline & & \multirow{2}{*}{ Aluminum } & & \multirow{2}{*}{101.6} & 0.8128 & 3048 & 2.5400 & $\begin{array}{l}\text { Yes } \\
\text { No }\end{array}$ & 5.55 & \\
\hline & & & & & 1.016 & 3048 & 2.5400 & $\begin{array}{l}\text { Yes } \\
\text { No }\end{array}$ & 7.75 & \\
\hline
\end{tabular}

continued on next page... 


\begin{tabular}{|c|c|c|c|c|c|c|c|c|c|c|}
\hline Source & Instruments & $\begin{array}{c}\text { Plate } \\
\text { Material }\end{array}$ & $\begin{array}{l}\text { Number } \\
\text { of Plates }\end{array}$ & $\begin{array}{c}\text { Plate } \\
\text { Width }\end{array}$ & $\begin{array}{c}\text { Plate } \\
\text { Thickness }\end{array}$ & $\begin{array}{c}\text { Plate } \\
\text { Length }\end{array}$ & $\begin{array}{l}\text { Channel } \\
\text { Thickness }\end{array}$ & Comb & $\begin{array}{c}\text { Calculated } \\
\text { Miller } \\
\text { Velocity }\end{array}$ & $\begin{array}{c}\text { Observed } \\
\text { Critical } \\
\text { Velocity }\end{array}$ \\
\hline & & & & $\begin{array}{c}b \\
(\mathrm{~mm})\end{array}$ & $\begin{array}{c}a \\
(\mathrm{~mm})\end{array}$ & $\begin{array}{c}L \\
(\mathrm{~mm})\end{array}$ & $\begin{array}{c}h \\
(\mathrm{~mm})\end{array}$ & & $\begin{array}{c}V_{C} \\
(\mathbf{m} / \mathbf{s})\end{array}$ & $(\mathbf{m} / \mathbf{s})$ \\
\hline \multirow{3}{*}{$\begin{array}{c}\text { Groninger } \\
(1963) \\
{[22]}\end{array}$} & \multirow{3}{*}{ strain gages } & \multirow{2}{*}{$\begin{array}{l}\text { Zircaloy with } \\
\text { fuel } \\
\text { compartments }\end{array}$} & \multirow{2}{*}{5} & 83.31 & $\begin{array}{c}1.6256 \\
(* 1.3970)\end{array}$ & 2196 & 1.9304 & $\begin{array}{l}\text { Yes } \\
\text { No }\end{array}$ & 13.11 & \\
\hline & & & & 83.31 & $\begin{array}{c}2.0066 \\
(* 1.9050)\end{array}$ & 2196 & 2.0066 & $\begin{array}{l}\text { Yes } \\
\text { No }\end{array}$ & 21.03 & \\
\hline & & $\begin{array}{c}\text { Solid } \\
\text { Zircaloy }\end{array}$ & 5 & 112.78 & 1.3208 & 2191 & 1.7780 & $\begin{array}{l}\text { Yes } \\
\text { No }\end{array}$ & 6.40 & \\
\hline \multirow{3}{*}{$\begin{array}{c}\text { Smissaert } \\
(1968) \\
{[23]}\end{array}$} & \multirow{3}{*}{$\begin{array}{c}\text { strain gages; } \\
\text { static } \\
\text { pressure }^{\dagger}\end{array}$} & \multirow{3}{*}{$\begin{array}{l}\text { polyvinyl- } \\
\text { chloride }\end{array}$} & 5 & 114.3 & 1.5875 & 1143 & 6.35 & $\begin{array}{c}\text { No } \\
\text { Yes }\end{array}$ & 2.59 & \\
\hline & & & 9 & 114.3 & 1.5875 & 1143 & 3.175 & $\begin{array}{c}\text { No } \\
\text { Yes }\end{array}$ & 1.81 & \\
\hline & & & 15 & 114.3 & 1.5875 & 1143 & 1.5875 & $\begin{array}{c}\text { No } \\
\text { Yes }\end{array}$ & 1.22 & \\
\hline $\begin{array}{c}\text { Ho } \\
(2004) \\
{[27]}\end{array}$ & $\begin{array}{c}\text { strain gages; } \\
\text { static } \\
\text { pressure; } \\
\text { visual }\end{array}$ & Aluminum & 2 & 78.2 & 1.2 & 780 & 4.3 & No & 15.4 & $11.9-12.0$ \\
\hline $\begin{array}{c}\text { Kennedy } \\
\text { (2015) }\end{array}$ & $\begin{array}{l}\text { static } \\
\text { pressure; } \\
\text { lasers; } \\
\text { visual }\end{array}$ & Aluminum & 1 & 110.29 & 1.016 & 647.7 & $2.032 / 2.540$ & $\begin{array}{l}\text { Yes } \\
\text { No }\end{array}$ & N/A & N/A \\
\hline
\end{tabular}

*This is the effective plate thickness if the plate was solid Zircaloy without fuel compartments

†Only in tests with $6.35 \mathrm{~mm}$ channels; $\neq$ Leading edge only 


\section{3: Numeric Modeling}

\subsection{1: CFD Turbulence}

In the analysis of fluid flow through a fuel assembly, several options exist for solving for the flow turbulence. The level of difficulty in solving complex, turbulent flow patterns is directly related to the level of detail and accuracy required in the final solution. While the ideal scenario would involve a complete solution of the Navier-Stokes equations, this is neither necessary nor an efficient use of current computational capabilities. Therefore, turbulent flow solutions are reliant upon models that approximate the energy losses associated with turbulent eddies in high Reynolds number flow [30]. Starting from the Navier-Stokes equations for the conservation of momentum in equation 2.25 , we can begin to approximate the impact of turbulent energy losses.

$$
\rho \frac{\partial u_{i}}{\partial t}+\rho \frac{\partial}{\partial x_{j}}\left(u_{j} u_{i}\right)=-\frac{\partial p}{\partial x_{i}}+\frac{\partial}{\partial x_{j}}\left(2 \mu s_{j i}\right)
$$

Assuming that a given turbulent flow varies randomly about some mean, the velocity components $u_{i}$ can be re-written in terms of their average values plus some component that randomly varies in time, as shown in equation 2.26.

$$
u_{i}(\boldsymbol{x}, t)=U_{i}(\boldsymbol{x})+u_{i}^{\prime}(\boldsymbol{x}, t)
$$

Combining equation 2.25 with 2.26 and rearranging yields the Reynolds Averaged Navier-Stokes (RANS) equation, equation 2.27. Equation 2.28 is the Reynolds stress tensor. 


$$
\begin{gathered}
\rho \frac{\partial U_{i}}{\partial t}+\rho U_{j} \frac{\partial U_{i}}{\partial x_{j}}=-\frac{\partial P}{\partial x_{i}}+\frac{\partial}{\partial x_{j}}\left(2 \mu S_{j i}-\overline{\rho u_{j}^{\prime} u_{l}^{\prime}}\right) \\
\tau_{i j}=-\overline{\rho u_{j}^{\prime} u_{l}^{\prime}}
\end{gathered}
$$

From equations 2.27 and 2.28, there are 10 unknown variables to solve for (six Reynolds stress components, $\tau_{i j}$, three velocity components, $u_{i}$, and pressure, $p$ ). Using the conservation of mass and the conservation of momentum (equation 2.27), only four equations exist to solve for the 10 unknown variables [31].

The lack of equations to solve for the remaining six variables is known as the closure problem. Numerous methods have been developed as ways to approximate the remaining variables. Over the past 50 years, as CFD modeling has grown in popularity, some of the most commonly used turbulence models take a two equation approach. The most popular turbulence models for closure of the RANS equations are the $k-\varepsilon$ and $k-\omega$. The variable $k$ in both of these models refer to the kinetic energy per unit mass of the turbulent fluctuations, and is defined by equation 2.29 [31].

$$
k=\frac{1}{2} \overline{u_{\imath}^{\prime} u_{\imath}^{\prime}}=\frac{1}{2}\left(u^{\prime 2}+v^{\prime 2}+w^{\prime 2}\right)
$$

The second variables refer to the turbulent dissipation per unit mass, $\varepsilon$, and the turbulent dissipation rate, $\omega$. The solution methods for these variables are far more complex, and multiple approximations have been developed. For the $k-\omega$ model in particular, a popular solution by Wilcox has been slightly modified and updated several times [31] [32]. An alternative solution by Menter seeks to use the $k$ - $\omega$ model near a wall, and blend into a $k$ $\varepsilon$ model in the free stream [33]. Since $k$ - $\omega$ has been shown to be more accurate for near 
wall flows and $k-\varepsilon$ is more accurate for free stream flows, the Menter technique is widely used in cases with a variety of flow conditions.

In CFD codes, two-equation turbulence models are dependent upon the Boussinesq approximation. This approximation assumes that the Reynolds stress tensor of equation 2.28 is proportional to the strain-rate tensor $S_{i j}$. This eddy viscosity approximation allows for estimation of turbulent energy losses in a flow without needing to actually solve for the turbulent eddies. Essentially, the losses as a result of turbulent eddies can be approximated as an additional viscosity term.

\subsection{2: Fluid-Structure Interaction}

In an effort to address mesh degradation in FSI simulations with large structural movements, Saksono proposed an adaptive remeshing strategy for a finite element representation of a fluid model. This technique has the secondary benefit of being able to capture interesting flow phenomenon in areas where high gradients develop, allowing for on-demand modification of the local mesh density [34]. While remeshing is an effective technique for maintaining quality CFD grids in FSI simulations, it often requires significant computational cost.

More recently, overset (chimera) grids have become a potential method for adapting a fluid mesh to large structural deflections. These grids utilize a background mesh and an overset mesh. The background mesh ignores the existence of the structure and fills the entire domain as though there was no structure present. The overset mesh is attached to the outer surface of the structure. In the direction extending perpendicular out from the 
structure, the mesh is typically at least four to six cells thick. The overset mesh is then laid on top of the background mesh. The outer layers of the overset mesh interpolate with the background mesh cells they overlap. Finally, any background mesh cells that are behind the structure and enclosed within the boundary of the interpolating cells, are cut out and not used in the simulation. As the structure moves, different cells in the background mesh are activated and deactivated, and the interpolating cells are also adjusted [35]. Additionally, the cell aspect ratios must be nearly equal between the grids, to ensure that there will be an adequate number of cells for the interpolation. Figure 2.5 is from a CD-Adapco presentation detailing overset grid interpolation methods in Star$\mathrm{CCM}+$, and illustrates the necessity for multiple layers of grid overlap [36].

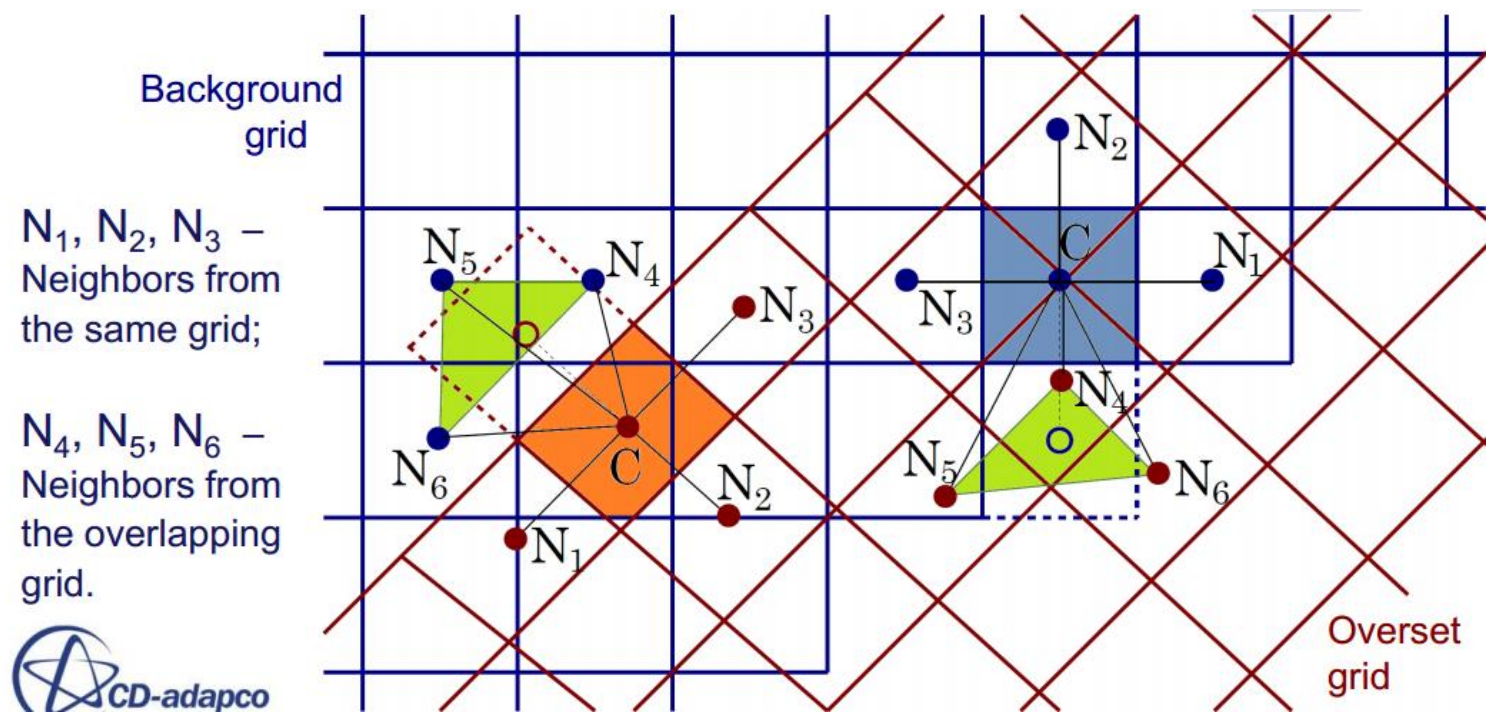

Figure 2.5. Star-CCM+ overset grid interpolation method

As numeric FSI capabilities evolved, the areas of application continue to expand. In medical research, flexible structures fluid channels have been coupled to pulsating fluid flow. This is allowing for investigation of blood flow in the case of a brain aneurysm [37]. In aerospace, simulations of a wing at various angles of attack continue to grow in 
complexity. More recent FSI methods range from analyzing the flapping an insect's wings to the deployment of a parachute [38].

One of the most significant difficulties with current FSI models is ensuring numeric stability while the simulation iterates. Simulation instability has been related to both the physical discretization (mesh) and the temporal discretization. In tightly coupled simulations, the range of stable time increments can be quite small. Particularly in cases where an incompressible fluid is coupled with a thin structure with relatively large deflections, there is an added mass effect of the fluid on the structure. This added mass effect tends to increase numeric instability [39]. In addition to the impact of discretization on stability, the velocity of the deforming interface can also be significant [40].

\subsection{3: Quantification of Numeric Uncertainty}

Like experiments, there is some uncertainty associated with numeric model results. The uncertainty seen in experiments arises primarily from the precision of the instrumentation and the variation in the physical processes being measured. In numeric models, similar uncertainties arise from the solvers and the discretization of the model. Therefore, it is important to study how the solution changes as the mesh is refined. This information can then be used to estimate the uncertainty associated with the finer mesh solution.

For a CFD grid, uncertainty is commonly established through a Richardson extrapolation. To get the final grid convergence index (GCI), the result of equation 2.30 is multiplied by 
a factor of safety. This factor is typically 3 for simple (2-grid) studies, and 1.25 if more than 2 grids are studied. The final GCI is shown in equation 2.34 [41].

$$
\begin{gathered}
E_{1}^{\text {fine }}=\frac{\varepsilon_{21}}{1-r^{p}} \\
p=\frac{\ln \left(\frac{\varepsilon_{32}}{\varepsilon_{21}}\right)}{\ln r} \\
h=\left[\frac{1}{N} \sum_{i=1}^{N} \Delta V_{i}\right]^{\frac{1}{3}} \\
\varepsilon_{32}=\phi_{3}-\phi_{2} \\
\varepsilon_{21}=\phi_{2}-\phi_{1} \\
G C I_{1}^{\text {fine }}=F_{S}\left|E_{1}\right|
\end{gathered}
$$

Where: $\varepsilon_{21}=\phi_{2}-\phi_{1}$

$\phi_{1}=$ a fine-grid numerical solution obtained with grid spacing $h_{1}$

$\phi_{2}=$ a coarse-grid numerical solution obtained with grid spacing $h_{2}$

$r=$ refinement factor between the coarse and fine grid $\left(\mathrm{r}=h_{2} / h_{1}>1\right)$, and

$p=$ formal order of accuracy of the algorithm.

$F_{\mathrm{S}}=$ factor of safety

The use of a factor of safety allows running a single, representative grid study to account for the error in a larger study. This minimizes the number of extra simulations that must be run to quantify the grid uncertainty. In the case of studies involving wall bounded turbulent flow, the changes in mesh refinement should account for the selected wall solution method. For instance, if wall functions are used, all grids must contain points within the boundary layer. If wall functions are not used, and the boundary layer is to be discretized, then all meshes should properly discretize the boundary layer with comparable wall $y+$ values of 1 or less. It is recommended that the wall mesh remain 
relatively unchanged for a grid convergence study, with focus being on the core mesh.

Celik illustrates this calculation of CFD GCI through a simple four step process [42]. 


\section{CHAPTER 3: HYDRO-MECHANICAL FLOW LOOP}

\section{1: Flow Loop Description}

The University of Missouri Hydro-Mechanical Flow Loop (HMFL) was first constructed in 2008 to assist in analysis of thermal-hydraulic models of LEU based Molybdenum-99 targets [43]. In its original incarnation, the HMFL consisted of a reservoir, pump, minimal piping, and a basic data acquisition system. In the years since, three extensive rebuilds and countless other modifications and upgrades have expanded the capabilities and versatility of the HMFL. Today, while the HMFL has retained the original pump and reservoir, little else remains the same.

The current version of the HMFL in Figure 3.1 includes nine differential pressure transducers, a flow meter, and two laser-based optical deflection sensors. Additionally, the physical structure of the HMFL has been extensively updated to dampen vibration. The vibration dampening structure is constructed of a modular aluminum extrusion (80/20) and includes a scaffold frame that surrounds and supports the test section. This frame is fixed to two heavy, cast iron posts supporting the building. Finally, the HMFL utilizes a National Instruments LabView data acquisition and control system. In addition to recording data during a flow test, the system provides control of eight stepper motors for laser positioning and an electro-pneumatic valve for flow rate control. 


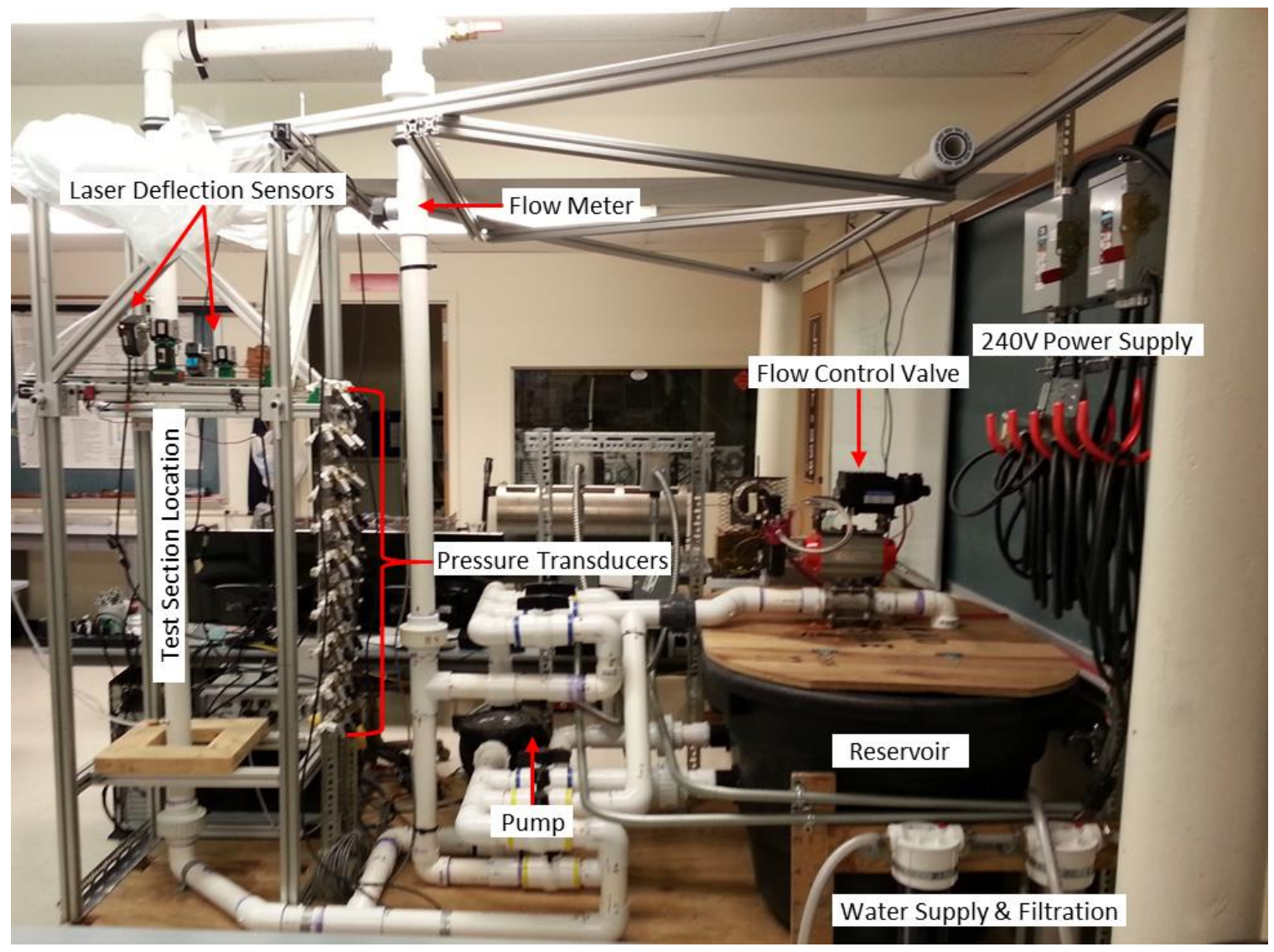

Figure 3.1. Hydro-Mechanical Flow Loop as of July 2014.

\section{2: Instrumentation}

\subsection{1: Flow Measurement}

During a flow test, the mass flow rate is determined by a frequency signal from an

Omega FP-7020 paddlewheel flow sensor. To convert the measured mass flow rate to a velocity, Eq. 3.1 is utilized.

$$
V=\frac{\dot{m}}{\rho A}
$$

Monitoring the flow rate is especially important since the open loop control for setting the flow rate prevents direct specification of a flow rate. While an effort is made to set 
the flow control valve so the system is near a desired flow rate, ultimately the flow meter data is the only reliable measure of the actual flow rate.

During post-processing, the flow meter is expected to provide frequency data no higher than $\sim 15 \mathrm{~Hz}$ at the highest flow rate. Occasionally, the data acquisition system records a value on the order of $100000 \mathrm{~Hz}$ or greater. These values are removed during postprocessing as they clearly represent a false signal.

\subsection{2: Pressure Measurement}

The flow loop is equipped with nine Omega PX-26 differential pressure transducers. When conducting flow tests on dual channel geometries, these pressure transducers are used to provide the pressure difference between the two channels at nine axial locations. This data provides the net pressure force on the plate. The pressure transducers are calibrated annually with a deadweight tester.

\subsection{3: Laser Based Channel Measurement}

The flow loop is equipped with two Keyence LK-G152 Laser Displacement sensors. The sensors work by emitting a laser beam and recording the location of the beam reflections. For the experiments described here, the outer walls of the test section are constructed of transparent Plexi-glass panels. This allows the laser to take measurements inside the test section in a non-obtrusive manner. During a flow test, the laser will receive multiple reflected signals corresponding to different reflection surfaces. This allows for relatively easy measurement of channel gaps during flow testing. 
One issue with the use of laser based sensors is the changing index of refraction of the mediums the laser is passing through. During a flow test, the laser signal passes through air, plexi-glass, and water. Because each of these has a different index of refraction, a calibration of the laser signal is completed each time the lasers are repositioned. To complete this calibration, the laser is first positioned in the desired location and two measurements are taken without any water in the test section. The first is the thickness of the Plexi-glass, $h_{1-2}$, and is represented by points 1 and 2 in Figure 3.2. The second measurement is of the channel gap, $h_{2-3}$, and is represented by points 2 and 3 in Figure 3.2. Assuming a uniform thickness of the plexi-glass, any refraction of the laser on its way into the Plexi-glass is negated by the reflected signal coming out of the plexi-glass. Therefore this measurement can be taken as the actual channel thickness.

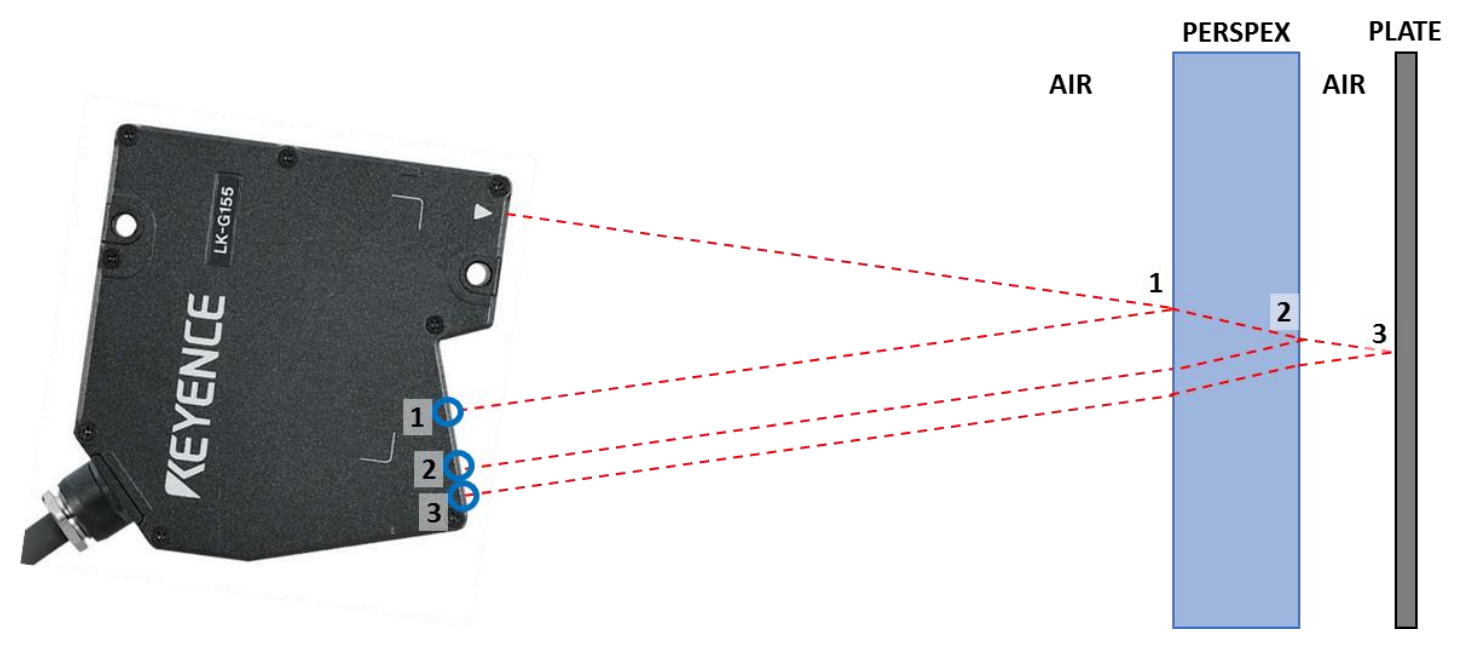

Figure 3.2. Laser measurement setup with air in the gap

After completing the measurements with air in the channels, the test section is filled with water and the final calibration measurement is taken. Once the channel is filled with water, the signal received from the inner surface of the plexi-glass disappears. This 
results from the water and plexi-glass having similar indices of refraction. Therefore for the final calibration measurement, as well as the experiments, the lasers are configured to measure the gap from the outside surface of the plexi-glass to the surface of the plate. This measurement, $h_{4-5}$, is represented by points 4 and 5 in Figure 3.3.

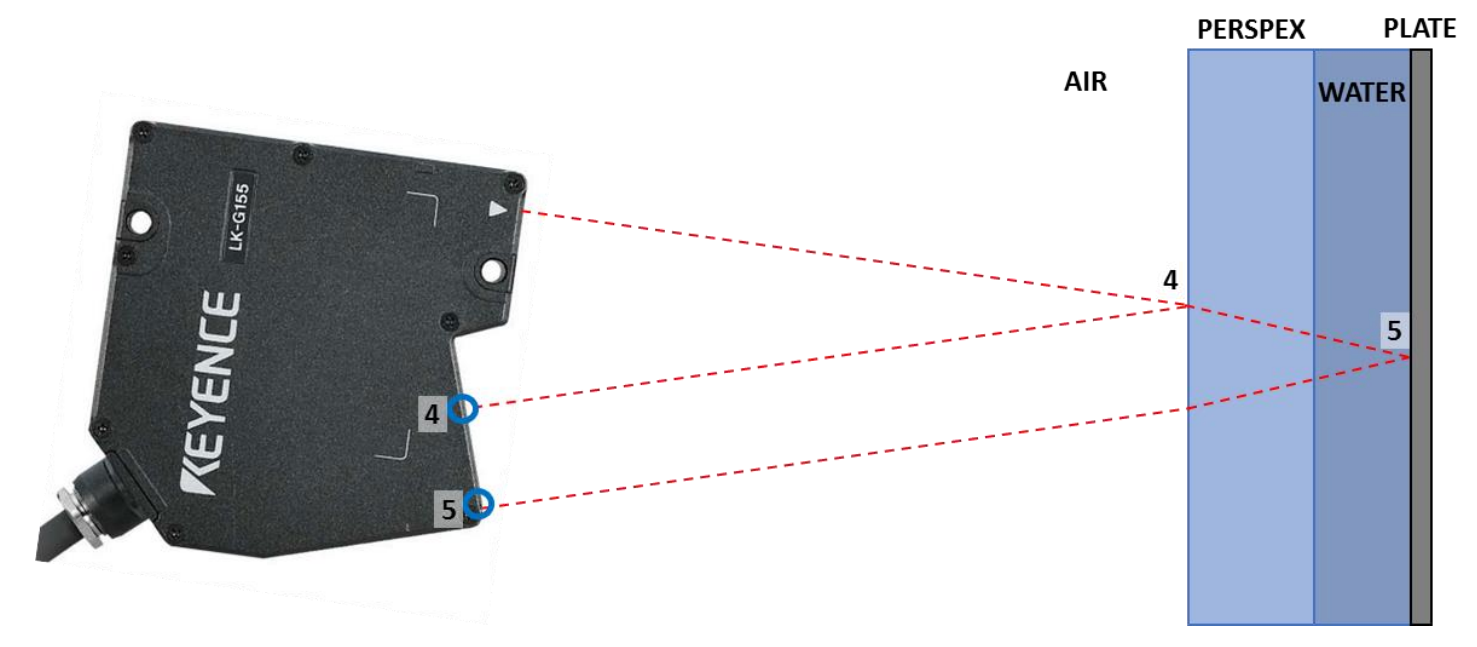

Figure 3.3. Laser measurement setup with water in the gap

Once the three calibration values $\left(h_{1-2}, h_{2-3}\right.$, and $\left.h_{4-5}\right)$ are obtained, a correction factor and calibration equation can be determined:

$$
\begin{gathered}
C_{L}=\frac{h_{2-3}}{h_{4-5}-h_{1-2}} \\
h_{R}=\left(h_{4-5}-h_{1-2}\right) * C_{L}
\end{gathered}
$$

Where:

$C_{L}=$ Laser correction factor

$h_{2-3}=$ Real gap thickness with air

$h_{4-5}=$ Measured plexi-glass + gap thickness with water

$h_{1-2}=$ Measured thickness of plexi-glass

$h_{R}=$ Real gap thickness after correction

A critical assumption of the calibration process is that the plexi-glass and channel

thickness does not vary over the angle of the laser reflection. During post-processing, the 
calibration equation is applied to all measured laser data to determine the actual channel

thickness. Additionally, any values registering a final gap measurement of less than 0 are discarded as they represent a loss of signal from the laser during data acquisition.

The lasers are positioned using stepper motors and are controlled through LabView using an Arduino. For the experiments presented here, the laser angle relative to the test section surface (rotation about $x$ ) and position across the width of the test section (translation in $x$ ) were controlled with the stepper motor system. Axial position (translation in y) was done by hand. Recently however, additional upgrades have been completed to allow for axial positioning with stepper motors as well. This upgraded system allows full, precise computer control of the laser positions for any future experiments.

\section{3: Data Acquisition \& Control System}

In order to collect data from the instruments and control certain flow loop functions, a robust data acquisition and control system has been constructed. This system, shown in Figure 3.4, integrates all of the data collection and control components of the flow loop. These components include power supplies, stepper motor drivers, laser sensor controller, an Arduino, and a National Instruments cDAQ. The cDAQ is the primary component of the system, and is shown in more detail in Figure 3.5. Some of the cDAQ capabilities are outlined in Table 3.1. 
Table 3.1. Available cDAQ measurements

\begin{tabular}{ccc} 
NI Number & Measurement & Channels \\
\hline 9237 & Half/Full Bridge Strain Gage & 4 \\
9236 & Quarter Bridge Strain Gage & 8 \\
9205 & 10V Analog Input & 16 \\
9213 & Thermocouple & 16 \\
9263 & 10V Analog Output & 4 \\
9225 & 300V Analog Input & 3 \\
9401 & Digital Input/Output & 8
\end{tabular}

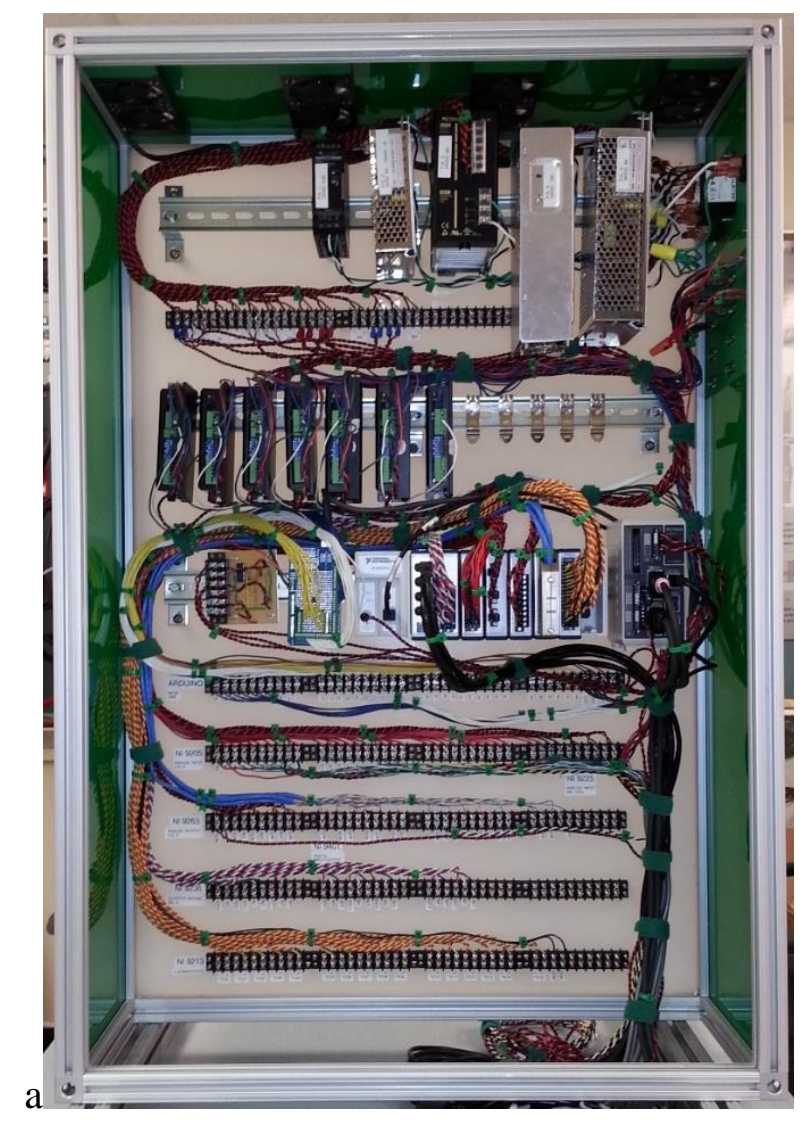

Figure 3.4. Data acquisition and control system 


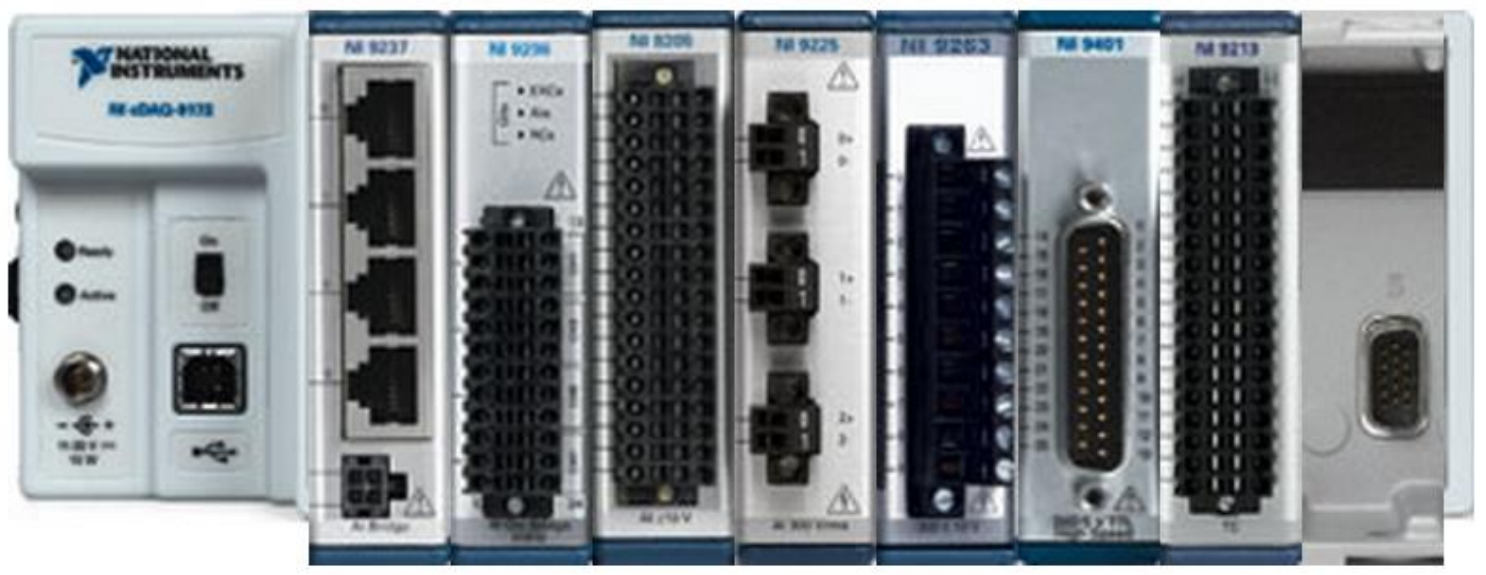

Figure 3.5. National Instruments cDAQ system

To control the components of this system, a LabView program is required. Several programs have been created for different types of experiments. The GUI for the most commonly used program is shown in Figure 3.6. This program allows for positioning of the lasers through use of stepper motors, positioning of a valve for flow rate control, and automated data acquisition from nine pressure transducers, one flow meter, and two laser displacement sensors. Data is typically collected at $1650 \mathrm{~Hz}$, providing a detailed, complete data set for post-processing and analysis. 


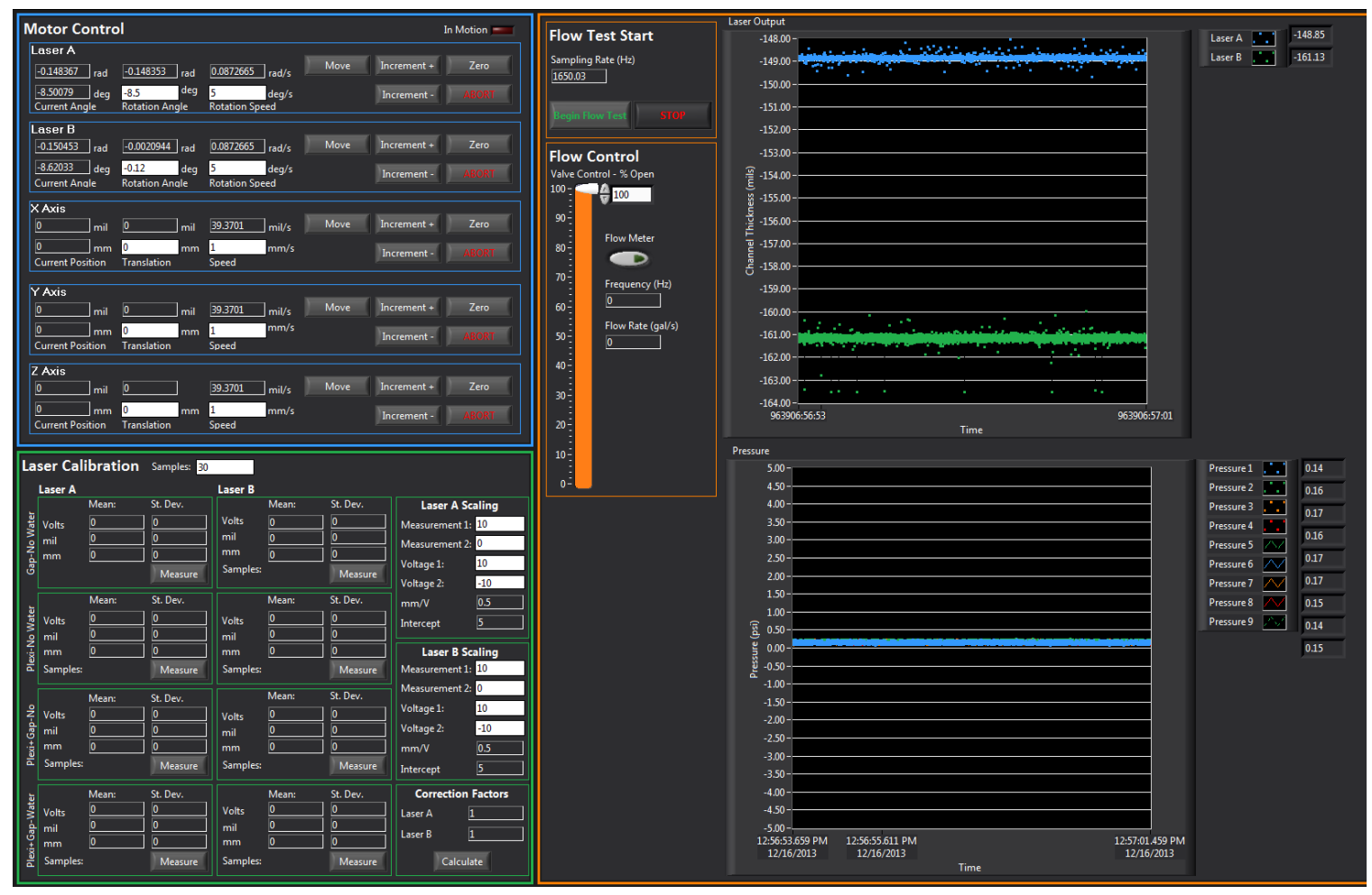

Figure 3.6. LabView graphical user interface 


\section{CHAPTER 4: EXPERIMENT SETUP}

\section{1: Experiment Objective}

In order to provide quality benchmark data for numeric model calibration, experiments on a single, flat plate test section have been completed. Previous experimental efforts have provided the basis for the experiments presented here. In a 2012 thesis, a set of experiments was completed and the results compared to a number of numeric FSI models [44]. Upon review of results from that study, there was noticeable difficulty in assessing the level of agreement between the experiments and the numeric models. These difficulties arose, in part, from an inadequate understanding of the actual experiment geometry.

In the experiment, it has been observed that there is some variation, or waviness, in the surface of the plexi-glass panels which make up the outer flow boundaries of the experiment. It is theorized that this variation has developed over time as a result of water absorption by the plexi-glass. Regardless of the source of the variation, it has a potentially significant effect on the uniformity of the fluid channel thicknesses. Additionally, while the plates would ideally be flat, they also exhibit some mild waviness once clamped into the test section. This also has an impact on the actual fluid channel uniformity and thicknesses. Therefore, in order to assess the impact of channel thickness variation, the present experiments include a mapping of the fluid channels.

In addition to mapping the channels prior to the experiments, this set of experiments also includes data collection at 11 flow rates with particular emphasis on average channel 
velocities between $4.5 \mathrm{~m} / \mathrm{s}$ and $6 \mathrm{~m} / \mathrm{s}$. This flow velocity range has historically been where the onset of significant plate bending was observed. Each flow rate sweep was repeated seven times to allow for placement of the channel gap-measuring lasers at seven different axial locations. This helps to develop a longitudinal profile of the plate at various flow rates. For collecting the laser data, previous experiments used a movingaverage approach. The present experiments are modified to take singular measurements with no averaging. This allows for future vibration analysis using a discrete Fourier transform.

For the present experiments, the test section was modified to include nine differential pressure measurements which are taken simultaneously. Rather than providing the pressure relative to ambient at one of 18 total locations among the two channels, this new system provides simultaneous measurements of the pressure differential between the two channels at nine axial locations. This allows for determining the net pressure difference experienced by the plate at a given axial location. Additionally, this process permits shorter testing run times and simpler post-processing.

Additional changes were made to increase the positioning accuracy and signal stability of the laser system. These changes, as shown in Figure 4.1, include the added benefit of generally stiffening the entire test section and greatly increasing the ability to dampen vibrations from the pump and flow loop. 


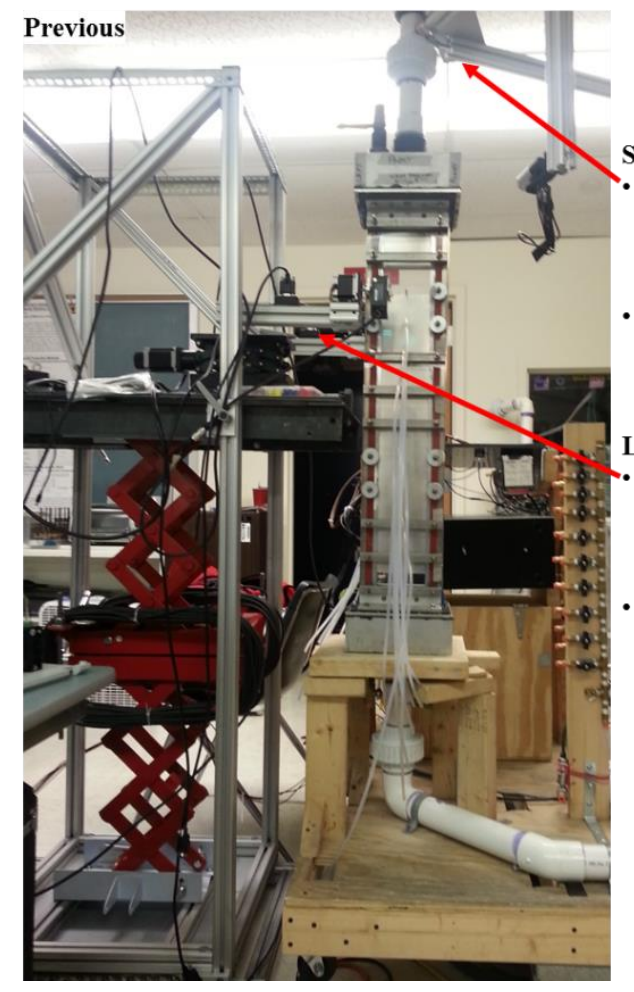

\section{Support Structure:}

Previous: Vibration dampening truss at top. Load from weight of truss carried by test section and piping.

- Current: Framing around test section. Load from upper vibration dampening truss now carried by framing. Structure is now stiffer. Laser Mounting:

- Previous: Laser stand to the side of the test section, with the lasers hanging cantilevered in front of the test section.

Current: Lasers mounted to frame surrounding test section. Provides more stable positioning than cantilevered approach.

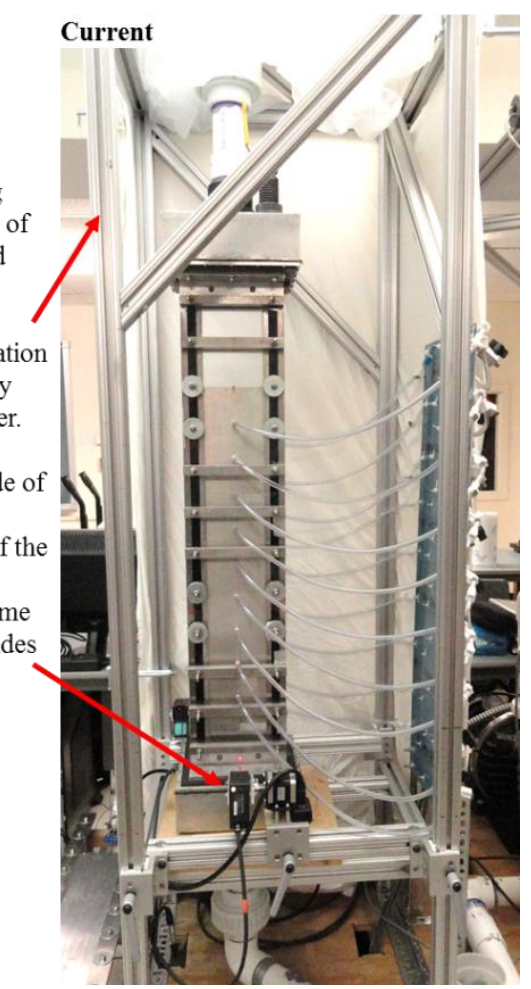

Figure 4.1. Overview of laser positioning and flow loop structural changes

With these changes, these experiments should provide a far more detailed description of the actual flow channel geometry. Additionally, by encompassing a greater number of flow rates and deflection measurement locations, these results represent the most comprehensive set of experiment benchmarking data to date.

\section{2: Test Section Design}

The test section consists of a single, flat plate with fluid channels on either side. Plate 23 of the current MURR HEU core provided a rough basis for the dimensions [45]. The long, vertical edges of the plate are clamped. Additionally, the fluid channels are intentionally made to be different sizes to simulate the type of plate offset that may be encountered during manufacturing of a fuel assembly. Figure 4.2 provides a horizontal 
cross-section of the test section and shows the strategy used for clamping the plate on its edges.

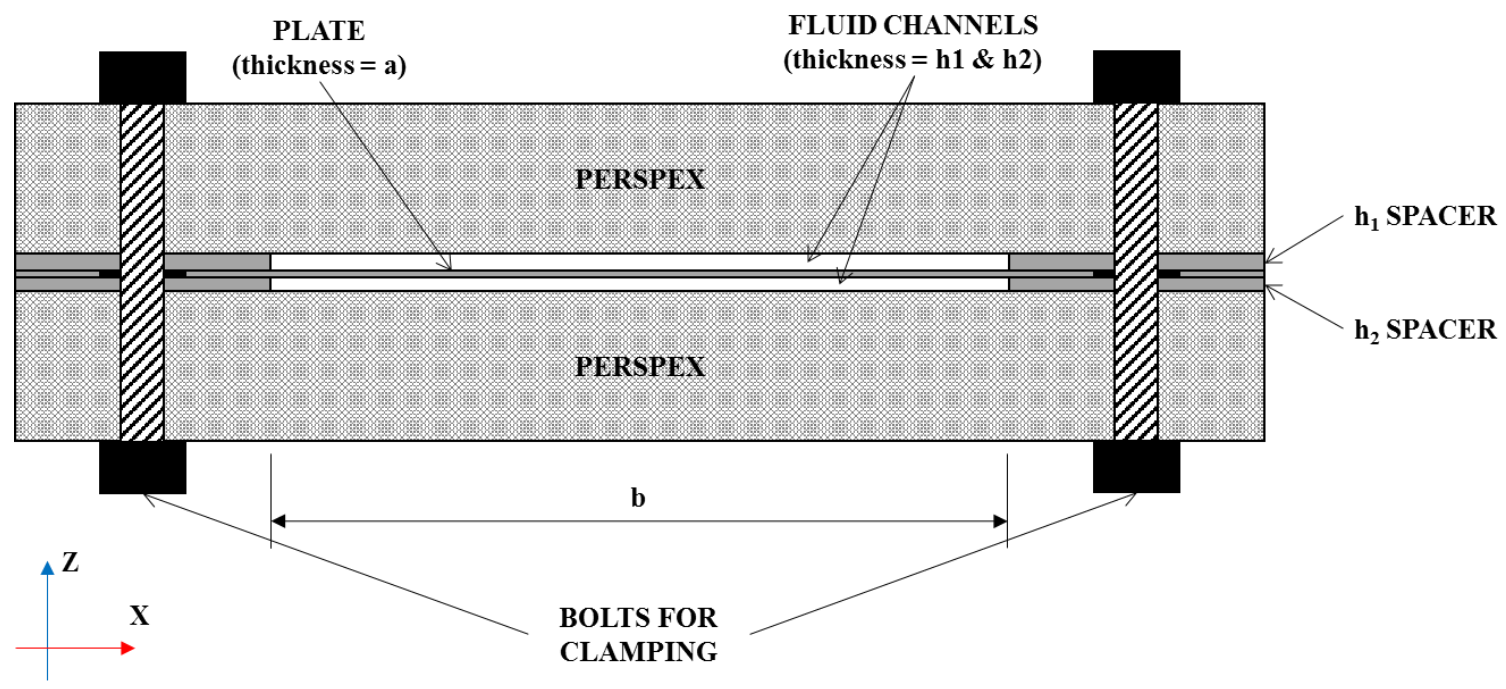

Figure 4.2. Test section horizontal cross-section (to scale)

The test section is also equipped with nine pressure taps in each plexi-glass panel. The taps are aligned along the axial centerline of the test section and spaced every $64.77 \mathrm{~mm}$ (2.55 inches) from the trailing edge of the plate to the leading edge. The exact pressure tap locations are provided in Table 4.1. Note that there are no pressure taps at the trailing and leading edges, as those locations are used for removable pins. The pins allow for fixing the midpoint of the leading and training edges of a plate, similar to a comb in a real fuel assembly. Figure 4.3 provides a schematic of the axial cross-section, with the locations of the pressure taps and pins noted. The pressure transducers are numbered from near the trailing edge of the plate $\left(\mathrm{PT}_{1}\right)$ to near the leading edge $\left(\mathrm{PT}_{9}\right)$. 


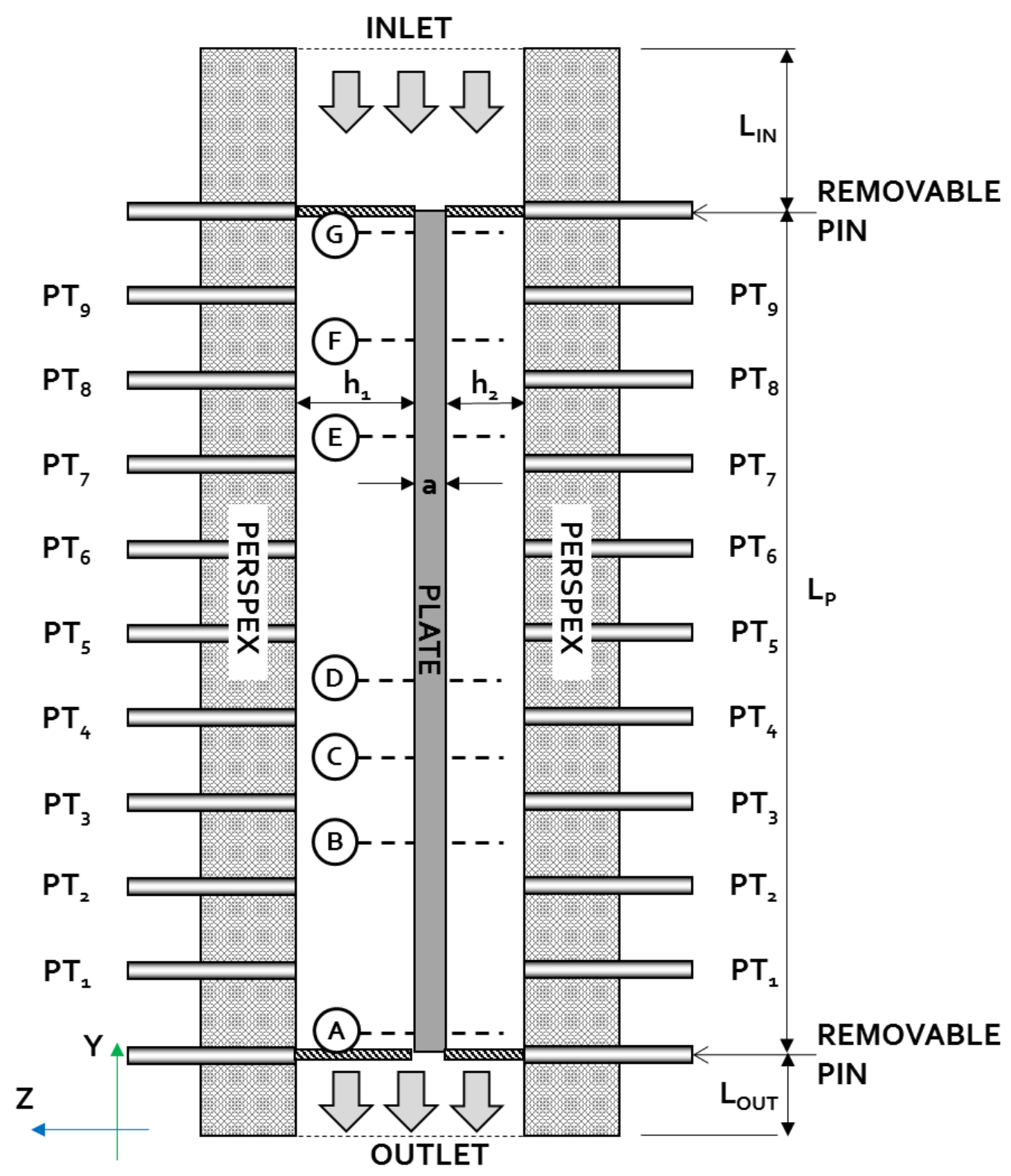

Figure 4.3. Test section vertical (axial) cross-section (not to scale) 
Table 4.1. Pressure tap locations

\begin{tabular}{ccc}
\hline Pressure Tap & \multicolumn{2}{c}{ Distance From Trailing Edge of Plate } \\
\hline $\mathbf{P T}_{\mathbf{1}}$ & $64.8 \mathrm{~mm}$ & $(2.55$ inches $)$ \\
$\mathbf{P T}_{\mathbf{2}}$ & $129.5 \mathrm{~mm}$ & $(5.10$ inches $)$ \\
$\mathbf{P T}_{3}$ & $194.3 \mathrm{~mm}$ & $(7.65$ inches $)$ \\
$\mathbf{P T}_{4}$ & $259.1 \mathrm{~mm}$ & $(10.20$ inches $)$ \\
$\mathbf{P T}_{5}$ & $323.9 \mathrm{~mm}$ & $(12.75$ inches $)$ \\
$\mathbf{P T}_{6}$ & $388.6 \mathrm{~mm}$ & $(15.30$ inches $)$ \\
$\mathbf{P T}_{7}$ & $453.39 \mathrm{~mm}$ & $(17.85$ inches $)$ \\
$\mathbf{P T}_{\mathbf{8}}$ & $518.2 \mathrm{~mm}$ & $(20.4$ inches $)$ \\
$\mathbf{P T}_{9}$ & $582.9 \mathrm{~mm}$ & $(22.95$ inches $)$ \\
\hline
\end{tabular}

The test section was designed to approximate a $1.016 \mathrm{~mm}$ (40 mil) plate offset by 0.254 $\mathrm{mm}(10 \mathrm{mils})$ in a fluid channel of $5.588 \mathrm{~mm}(220 \mathrm{mils})$. The width of the test plate is slightly larger than the arc length of any of the proposed MURR plates. Table 4.2 provides the designed dimensions for the parameters shown in Figure 4.2 and Figure 4.3.

Table 4.2. Designed test section dimensions

\begin{tabular}{ccc}
\hline Parameter & \multicolumn{2}{c}{ Design Value } \\
\hline $\mathbf{a}$ & $1.016 \mathrm{~mm}$ & $(0.040$ inches $)$ \\
$\mathbf{b}$ & $110.287 \mathrm{~mm}$ & $(4.342$ inches $)$ \\
$\mathbf{h}_{\mathbf{1}}$ & $2.540 \mathrm{~mm}$ & $(0.100$ inches $)$ \\
$\mathbf{h}_{\mathbf{2}}$ & $2.032 \mathrm{~mm}$ & $(0.080$ inches $)$ \\
$\mathbf{L}_{\mathbf{I N}}$ & $190.5 \mathrm{~mm}$ & $(7.5$ inches $)$ \\
$\mathbf{L}_{\mathbf{P}}$ & $647.7 \mathrm{~mm}$ & $(25.5$ inches $)$ \\
$\mathbf{L}_{\text {OUT }}$ & $76.2 \mathrm{~mm}$ & $(3$ inches $)$ \\
\hline
\end{tabular}


Once the test section was assembled, it was inserted into the flow loop by connecting the inlet and outlet pipes. The pressure lines were also connected and the lasers were positioned on either side of the test section. The completed test section is shown in Figure 4.4 with key features identified.

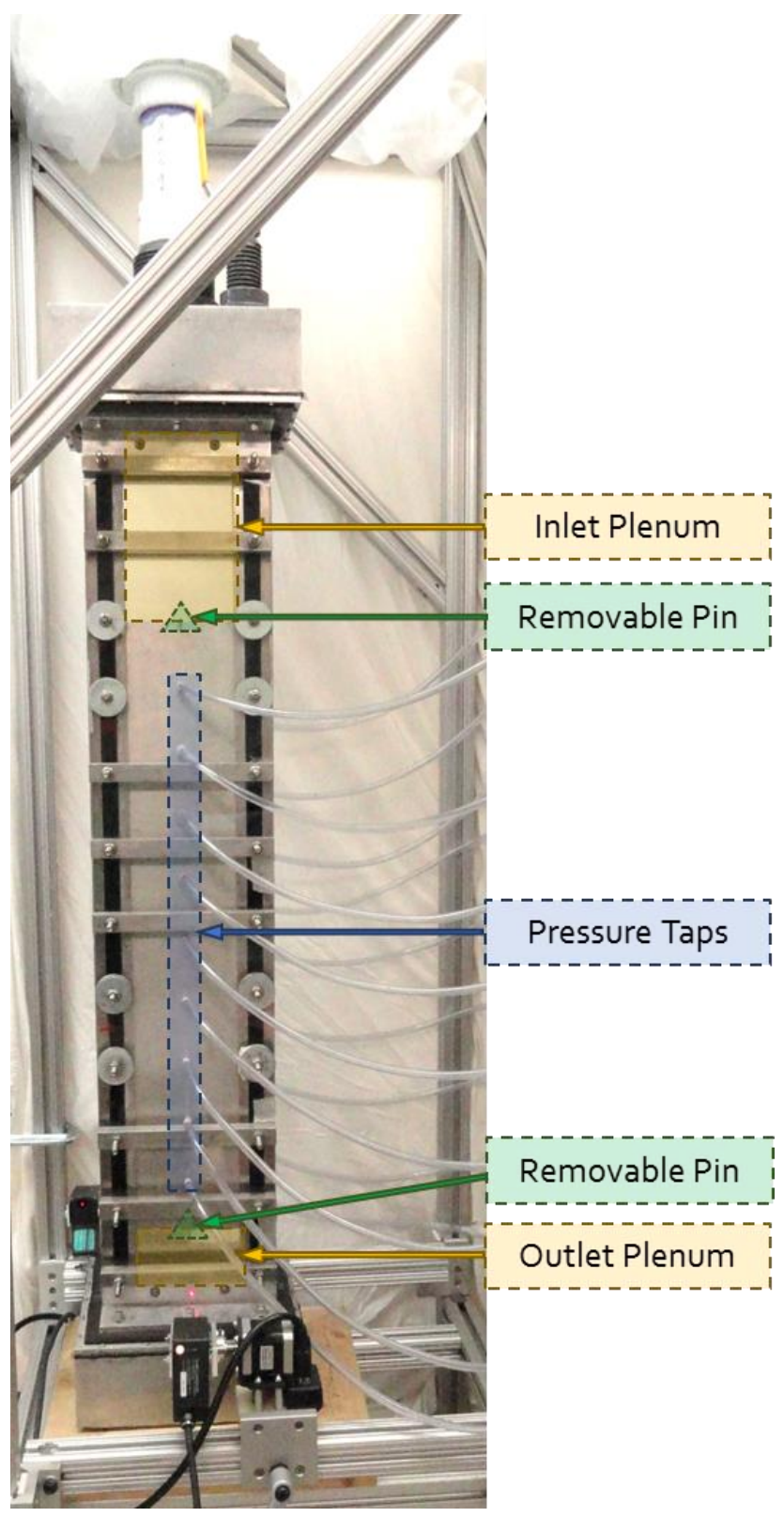

Figure 4.4. Test section ready for flow testing 


\section{3: Channel Mapping}

The fluid channel gaps in the test section, while targeted to be $2.032 \mathrm{~mm}$ (80 mils) and $2.540 \mathrm{~mm}$ (100 mils), will vary from those values as illustrated in Figure 4.5. The two primary factors contributing to variation in the fluid channel thickness are inconsistencies in the smoothness of the Plexi-glass and variations in the flatness of the plate. Therefore, in order to better characterize the geometry of the channels, the laser displacement sensors were utilized to map the thickness of the fluid channels.

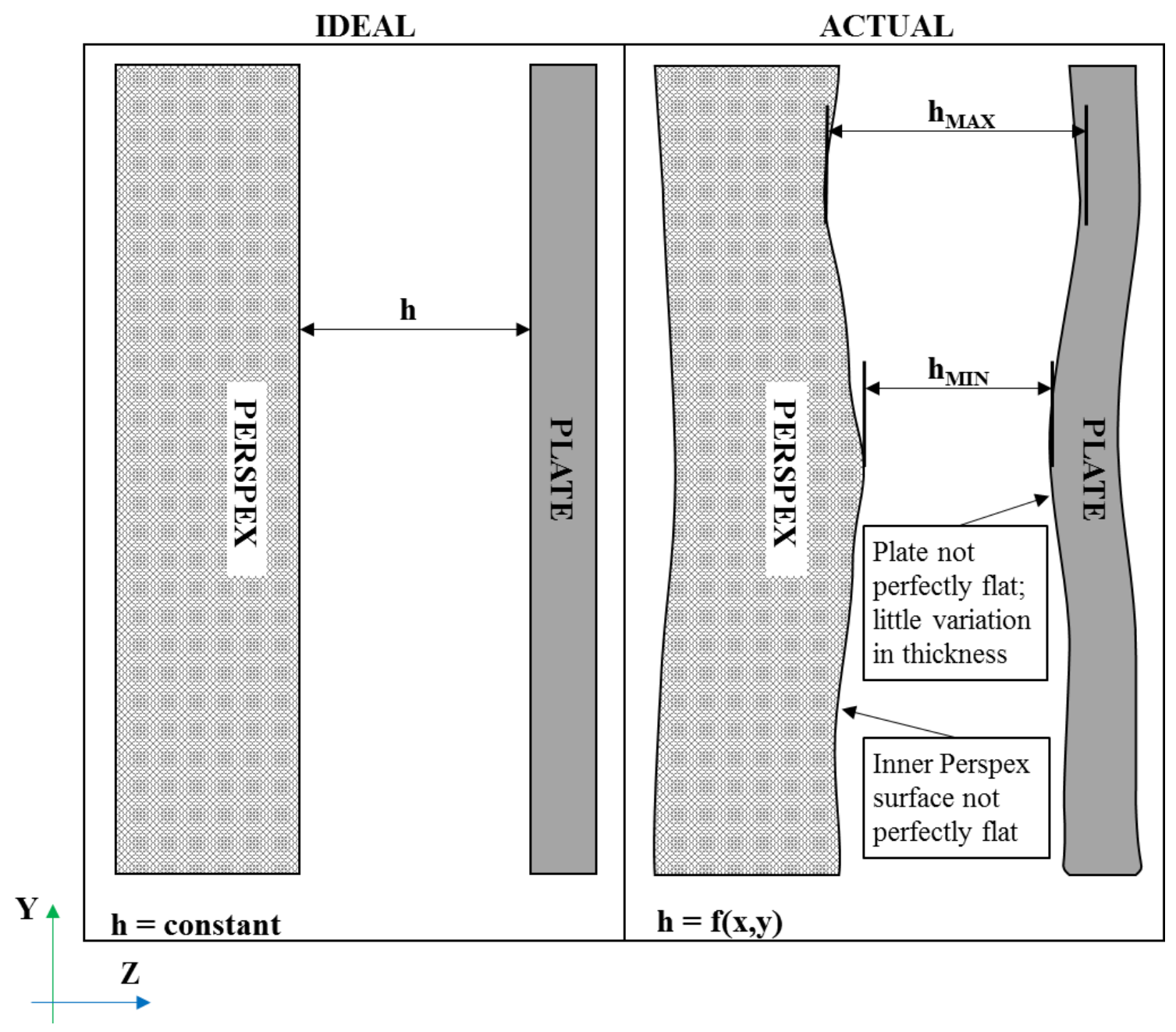

Figure 4.5. Ideal channel thickness vs. actual thickness 
The laser displacement sensors are capable of high measurement accuracy and are designed to be moved to nearly any location on test section surface. Therefore, the lasers were used to perform repeated mappings of the channels. The initial channel mapping consisted of taking measurements at 120 locations in each channel. The measurement grid consisted of 12 locations across the width (x-direction) of the plate, and 10 locations along the length (y-direction) of the plate. Each measurement involved taking 30 samples from each laser, and the process was repeated for a total of nine trials. This information is useful not only for analyzing the actual shape of the channels, but the repeated trials of the mapping are useful for determining the error associated with repositioning of the lasers. The basic grid used for the initial channel mapping is shown in Figure 4.6. 


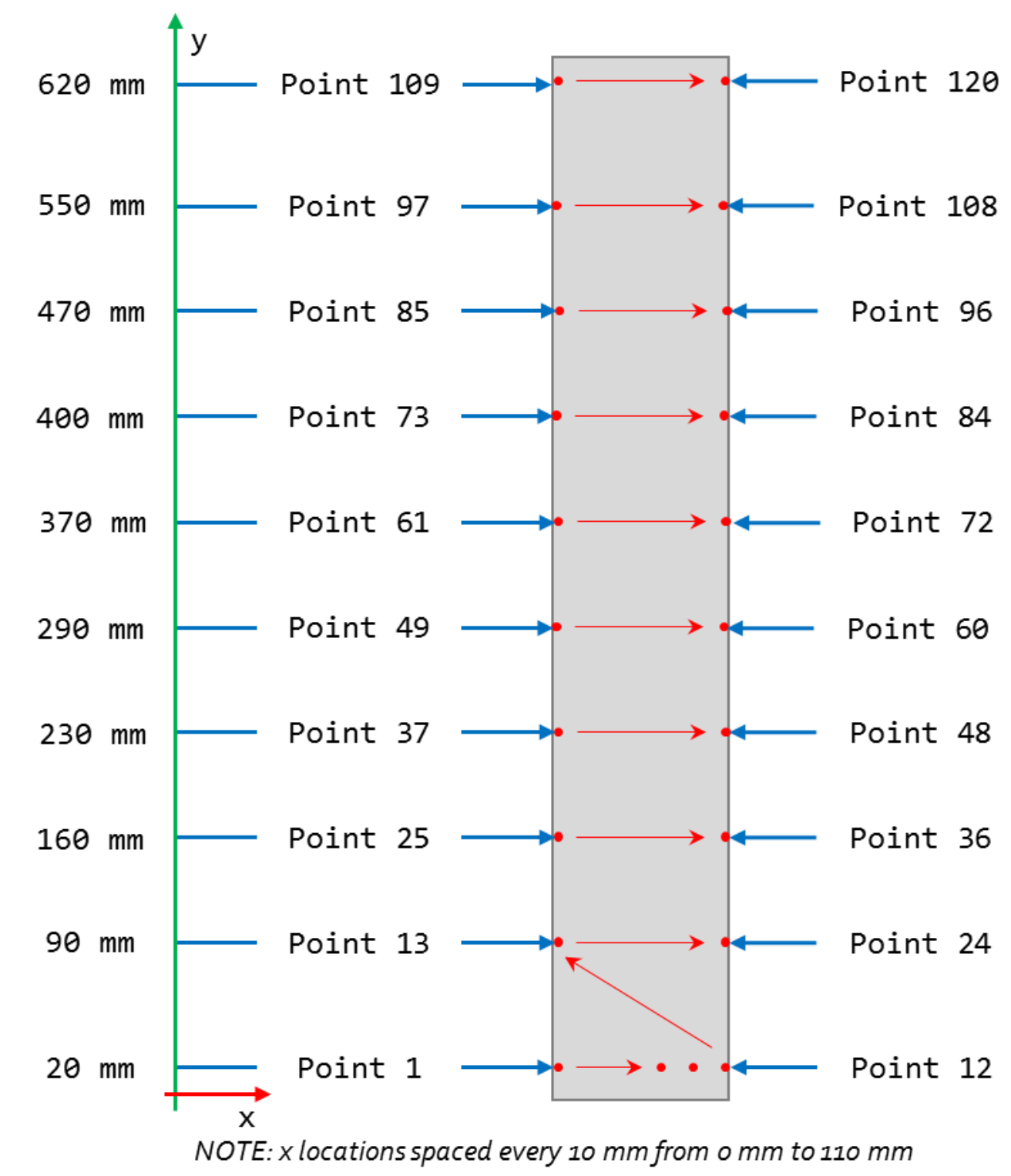

Figure 4.6. Channel mapping grid.

As will be shown in the next chapter, there was significant variability in the repeated channel measurements. Examination of the mapping process revealed that this variability is likely a result of inaccuracies in repositioning the lasers for each trial. When positioning the lasers in the axial direction, it was especially difficult to target the same location each time. In order to increase accuracy in positioning the lasers, additional 
controls were needed to minimize the uncertainty in manual positioning. To this end, a number of upgrades were completed to expand the capabilities of the laser positioning system. Four stepper motors were added to allow for digital control of the laser axial location. Also, a new LabView program allowed full automation of the mapping process. This upgraded system allowed for a measurement grid with thousands of measurement locations, with little to no user interaction. Figure 4.7 shows two of the new positioning motors.

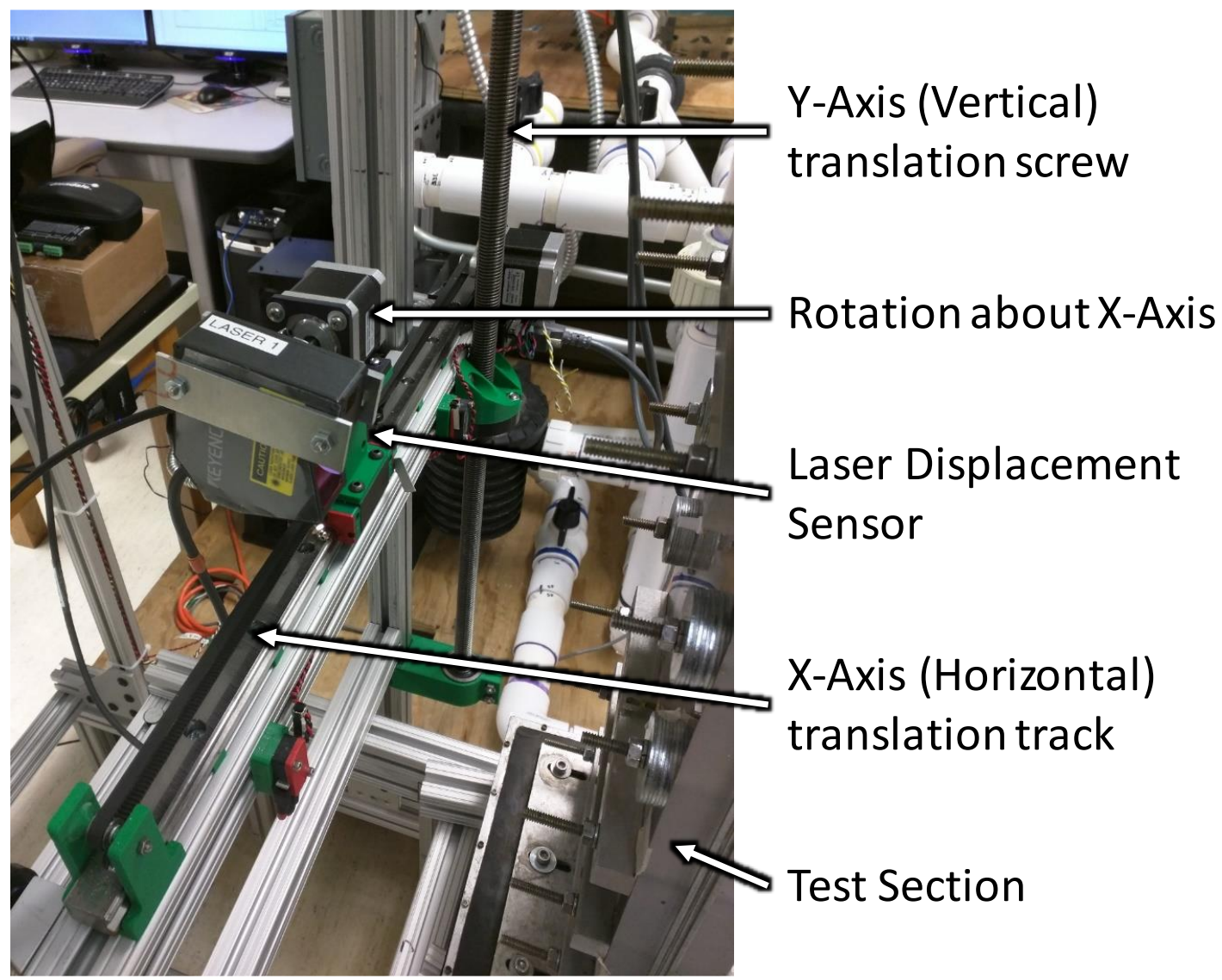

Figure 4.7. Laser axial positioning upgrades 


\section{4: Flow Test Procedure}

After completing the channel mapping study, flow testing could begin. The basic procedure for completing the experiments was:

1. Position the Lasers: Position the lasers at the desired axial and horizontal location. Rotate the lasers down $8.5^{\circ}$ from perpendicular to the test section.

2. Collect Calibration Data: Collect the laser calibration data as outlined in Eqns. 3.2 and 3.3.

3. Bleed the Pressure Lines: With the water flowing at the lowest flow rate, bleed air out of all the pressure lines.

4. Begin Data Collection: Begin with the lowest flow rate, collect data for approximately 30 seconds at a sampling frequency of $1650 \mathrm{~Hz}$, and then move on to the next flow rate.

Repeat at Next Laser Position: Repeat this process until data at all laser positions (A-G in Figure 4.3 and Table 4.3) and plate edge conditions (free/combed) has been recorded.

The experiments were completed with the leading and trailing edges both free and

pinned. The lasers were located at seven axial locations, as illustrated in Figure 4.3. The laser locations from the trailing edge of the plate are provided in Table 4.3. 
Table 4.3. Laser axial measurement distance from plate trailing edge

\begin{tabular}{ccc}
\hline Location ID & \multicolumn{2}{c}{ Axial Location (From Plate Trailing Edge) } \\
\hline A & $12.7 \mathrm{~mm}$ & $(0.500$ inches $)$ \\
B & $152.4 \mathrm{~mm}$ & $(6.000$ inches $)$ \\
C & $226.7 \mathrm{~mm}$ & $(8.925$ inches $)$ \\
D & $290.5 \mathrm{~mm}$ & $(11.438$ inches $)$ \\
E & $474.3 \mathrm{~mm}$ & $(18.675$ inches $)$ \\
F & $550.5 \mathrm{~mm}$ & $(21.675$ inches $)$ \\
G & $635.0 \mathrm{~mm}$ & $(25.000$ inches $)$ \\
\hline
\end{tabular}

The flow rate is controlled with an open loop system. The flow is set by controlling the position of a flow bypass valve. With the valve fully open, the test section sees its lowest flow rate. With the valve fully closed, all flow from the pump is directed through the test section. While the user can not directly specify a flow rate when setting the valve position, they can view the output from the flow meter in real time. This gives the user a reasonable approximation of the flow rate.

Prior to conducting the initial flow test, a set of target velocities was determined. These velocities were based on the capabilities of the flow loop as well as an estimation of the velocities seen at MURR. Using those velocities in conjunction with the mapped average channel area in Table 5.1 and Eq. 3.1, the approximate mass flow rates were determined. Table 4.4 provides those targeted values. Note that much like the ideal values for channel thickness, the actual experiment will deviate from these specified values. The data recorded with the flow meter will provide the actual flow rates in the experiments, and those actual flow rates will be used for all post-processing. During the initial flow 
test, the bypass valve positions which appeared to best provide the desired flow rates were recorded. Those valve positions were used in all later experiments in an effort to provide the closest flow rate matching possible between experiments. The actual, measured flow rates can be found in sections 5.3.1 and 5.4.1. These sections also provide the fluid velocities based on the measured average total area of the fluid channels, as provided in Table 5.1. If a given measured flow rate were applied to an ideal geometry rather than the measured experiment geometry, Eq. 4.1 can be used to find a conversion ratio based on the relative ratio of the areas.

Table 4.4. Targeted velocities and flow rates for the initial flow test

\begin{tabular}{lll}
\hline $\mathbf{V}(\mathbf{m} / \mathbf{s})$ & $\dot{\mathbf{m}}(\mathbf{k g} / \mathbf{s})$ \\
\hline $\mathbf{2 . 0}$ & 1.009 \\
$\mathbf{3 . 0}$ & 1.513 \\
$\mathbf{4 . 0}$ & 2.018 \\
$\mathbf{5 . 0}$ & 2.522 \\
$\mathbf{5 . 3}$ & 2.673 \\
$\mathbf{5 . 6}$ & 2.825 \\
$\mathbf{5 . 9}$ & 2.976 \\
$\mathbf{6 . 0}$ & 3.026 \\
$\mathbf{7 . 0}$ & 3.531 \\
$\mathbf{8 . 0}$ & 4.035 \\
$\mathbf{9 . 0}$ & 4.540 \\
\hline
\end{tabular}




$$
\frac{V_{I D E A L}}{V_{M E A S}}=\frac{A_{M E A S}}{A_{I D E A L}}=\frac{505.88 \mathrm{~mm}^{2}}{504.231 \mathrm{~mm}^{2}}=1.00327
$$

From Eq. 4.1 it is clear that the average experiment velocity is very close to what would be expected if there were no variations in the channel thicknesses. When applied to the channels independently, the ideal to measured area ratios are 0.962 and 1.055 for the larger and smaller channels, respectively. Since the flow distribution between the channels is dependent upon additional factors, the independent channel area ratios are likely not indicative of velocity deviations from ideal in the specific channels. 


\section{CHAPTER 5: EXPERIMENT RESULTS}

\section{1: Channel Mapping}

\subsection{1: Initial (Semi-Manual) Mapping}

When first assessing the thickness of the fluid channels in the experiment, the apparatus required manual movement of the lasers in the $\mathrm{y}$ (vertical) direction, while the $\mathrm{x}$ (horizontal) direction was controlled via an Arduino/LabView system. This setup required the researcher to carefully position the lasers vertically every time a new location was to be measured. In order to assess the accuracy of this positioning process, the process was repeated several times, allowing for assessment of the ability to reproduce measurement results.

By repeating the mapping process nine times, it is possible to begin to understand the errors associated with the placement of the laser sensors. To understand the variation within each mapping, Figure 5.1 shows the mean values at each measurement location, with error bars representing the minimum and maximum measured values. The $\mathrm{x}$-axis corresponds to the measurement locations shown in Figure 4.6, and therefore each set of 12 points corresponds to one horizontal row on the test section. The average channel thicknesses and areas are provided in Table 5.1. 


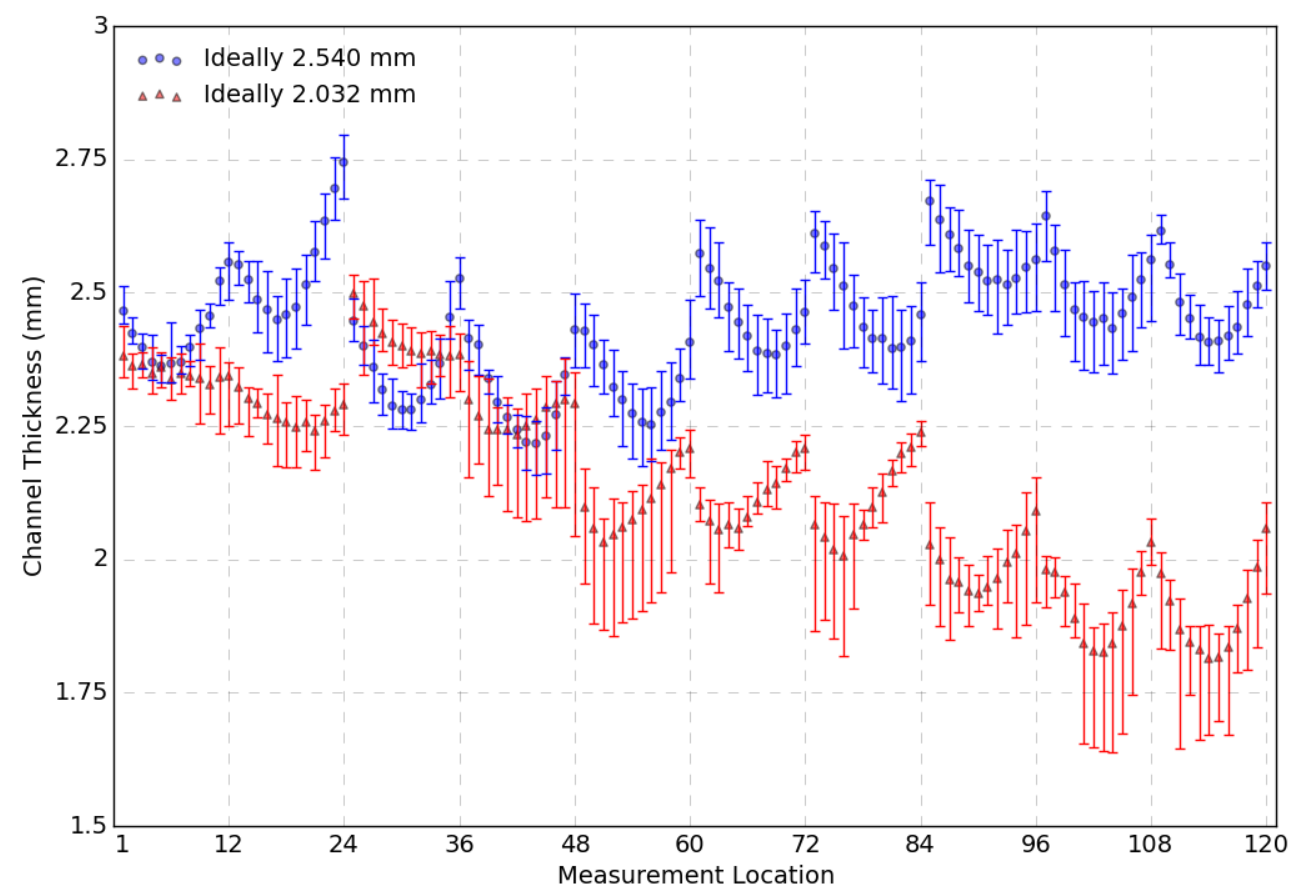

Figure 5.1. Channel gap mean, minimum, and maximum values.

Table 5.1. Ideal and measured channel thickness and area

\begin{tabular}{c|cccc|cccc}
\hline \multirow{2}{*}{ Channel } & \multicolumn{3}{|c}{ Channel Thickness } & \multicolumn{4}{c}{ Channel Area } \\
& \multicolumn{2}{|c}{ Ideal } & \multicolumn{2}{c}{ Measured } & \multicolumn{2}{c}{ Ideal } & \multicolumn{2}{c}{ Measured } \\
& $\mathrm{mm}$ & inch & $\mathrm{mm}$ & inch & $\mathrm{mm}^{2}$ & $\mathrm{in}^{2}$ & $\mathrm{~mm}^{2}$ & $\mathrm{in}^{2}$ \\
\hline $\mathbf{1}$ & 2.540 & 0.100 & 2.444 & 0.0962 & 280.128 & 0.4342 & 269.52 & 0.4178 \\
$\mathbf{2}$ & 2.032 & 0.080 & 2.143 & 0.0844 & 224.103 & 0.3474 & 236.35 & 0.3664 \\
\multirow{2}{*}{ Total } & $\mathbf{4 . 5 7 2}$ & $\mathbf{0 . 1 8 0}$ & $\mathbf{4 . 5 8 7}$ & $\mathbf{0 . 1 8 0 6}$ & $\mathbf{5 0 4 . 2 3 1}$ & $\mathbf{0 . 7 8 2}$ & $\mathbf{5 0 5 . 8 8}$ & $\mathbf{0 . 7 8 4 1}$ \\
\hline
\end{tabular}

The average of the nine measurements taken at a given location was calculated and used to generate surface plots of the channel thicknesses. For the nine trials completed before the flow experiments, Figure 5.2 shows the channel thicknesses. In addition to the 
channel thicknesses, it is helpful to know the differential in the channel thicknesses and the sum of the thicknesses. Figure 5.3 shows these values. Note that in the channel difference plot, the ideally larger channel is actually smaller in an area approximately 225 $\mathrm{mm}$ from the trailing edge of the plate. Additionally, from the channel summation plot it is clear that the channels tend to become thinner near the axial centerline of the channels. This observation has been confirmed visually during assembly of the test section, with the bowing of the panels being easily visible. 


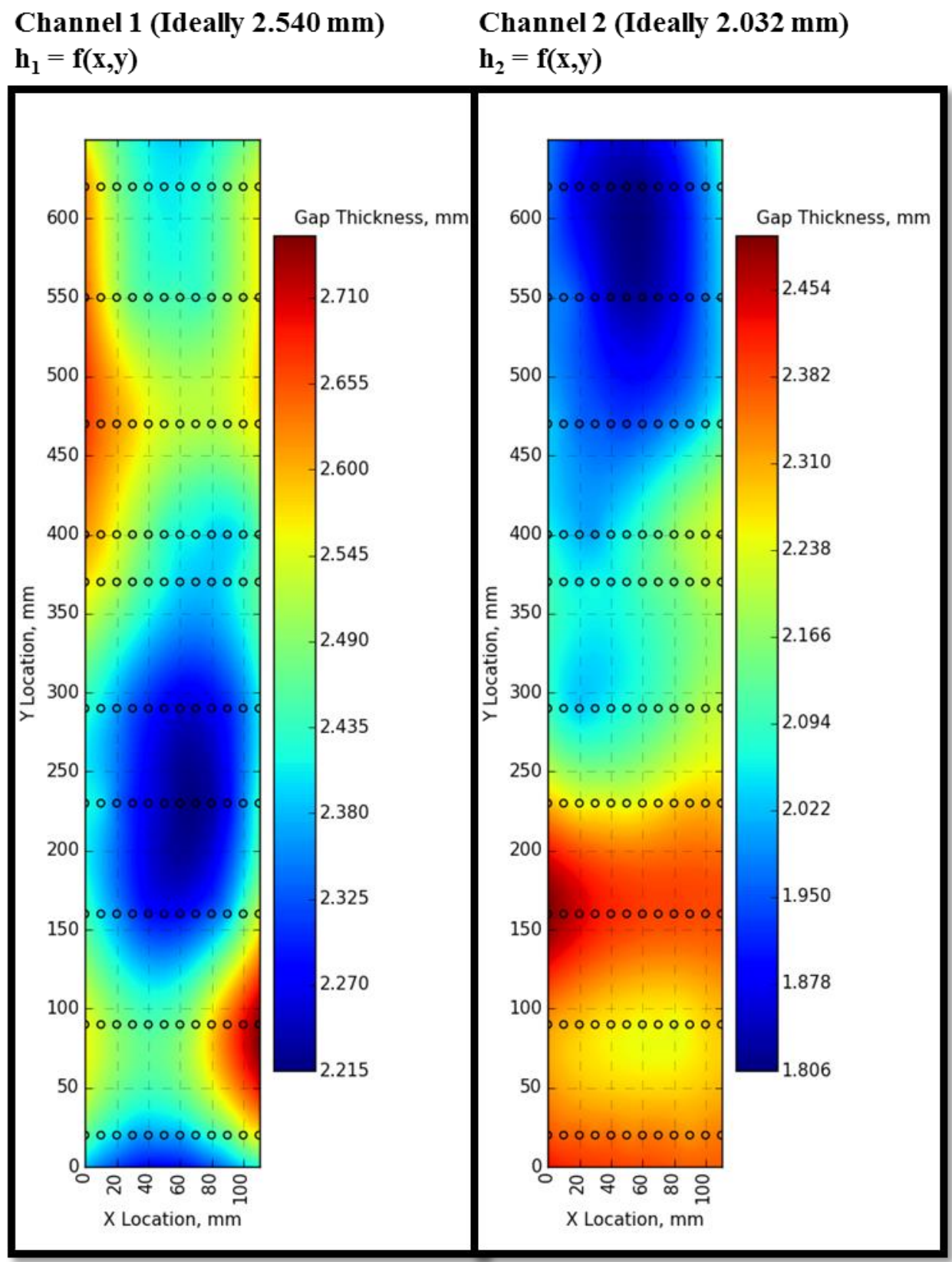

Figure 5.2. Low density channel thickness mapping 


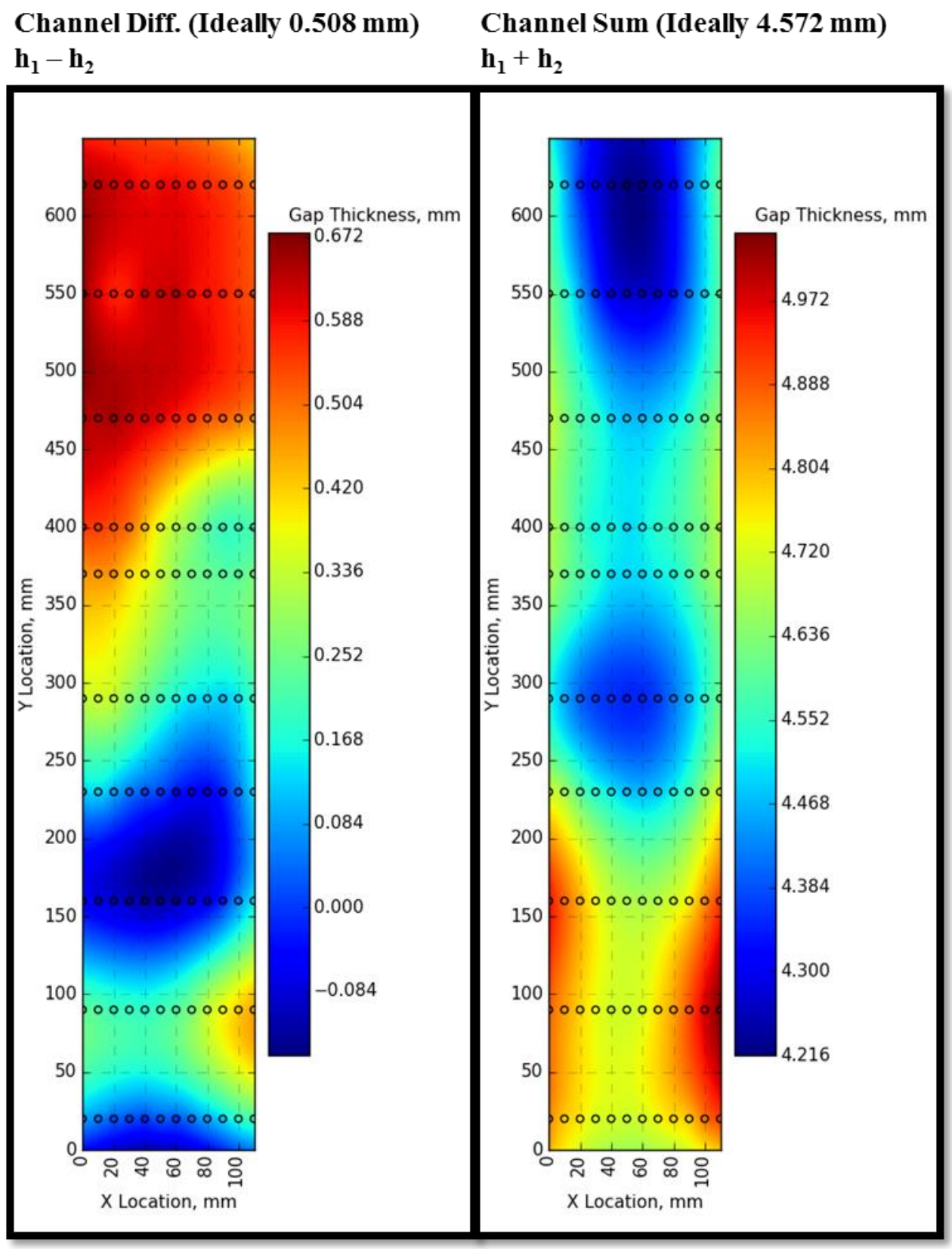

Figure 5.3. Low density channel difference and sum

The wide variation in measured channel thickness seen in Figure 5.1 is likely the result of inaccuracies in positioning the lasers. As was mentioned earlier, the lasers are positioned with stepper motors in the horizontal (X) direction and manually in the vertical (Y) direction. 


\subsection{2: Upgraded (Fully Automatic) Mapping}

Using the upgraded, fully automatic mapping system, it was possible to complete a more detailed mapping of the fluid channels. A LabView program and an Arduino were utilized to automate the mapping process, with the lasers starting each pass at the top of the plate, working across the plate width, and then moving down. Upon reaching the final location at the bottom of the plate, the lasers were reset at the top edge and the process was repeated a total of 30 times. Some locations were obstructed from view of the lasers and no data is available in those areas. A grid of roughly $5 \mathrm{~mm}$ was used for the mapping, with a total of 2000 locations in Channel 1 and 2026 locations in Channel 2 being measured.

The average of all the channel mapping passes was used to generate the color map of Figure 5.4. A linear interpolation was used to approximate the channel thickness between measurement locations. Through all the mapping trials, the measured thickness of channel 1 was repeatable with a $95 \%$ uncertainty of $\pm 0.0060 \mathrm{~mm}$, and channel 2 was repeatable with a $95 \%$ uncertainty of $\pm 0.0350 \mathrm{~mm}$. The reason for the discrepancy in the uncertainty values may be the result of tolerances in the positioning system. Summary data for the channel gaps is shown in Table 5.2. 

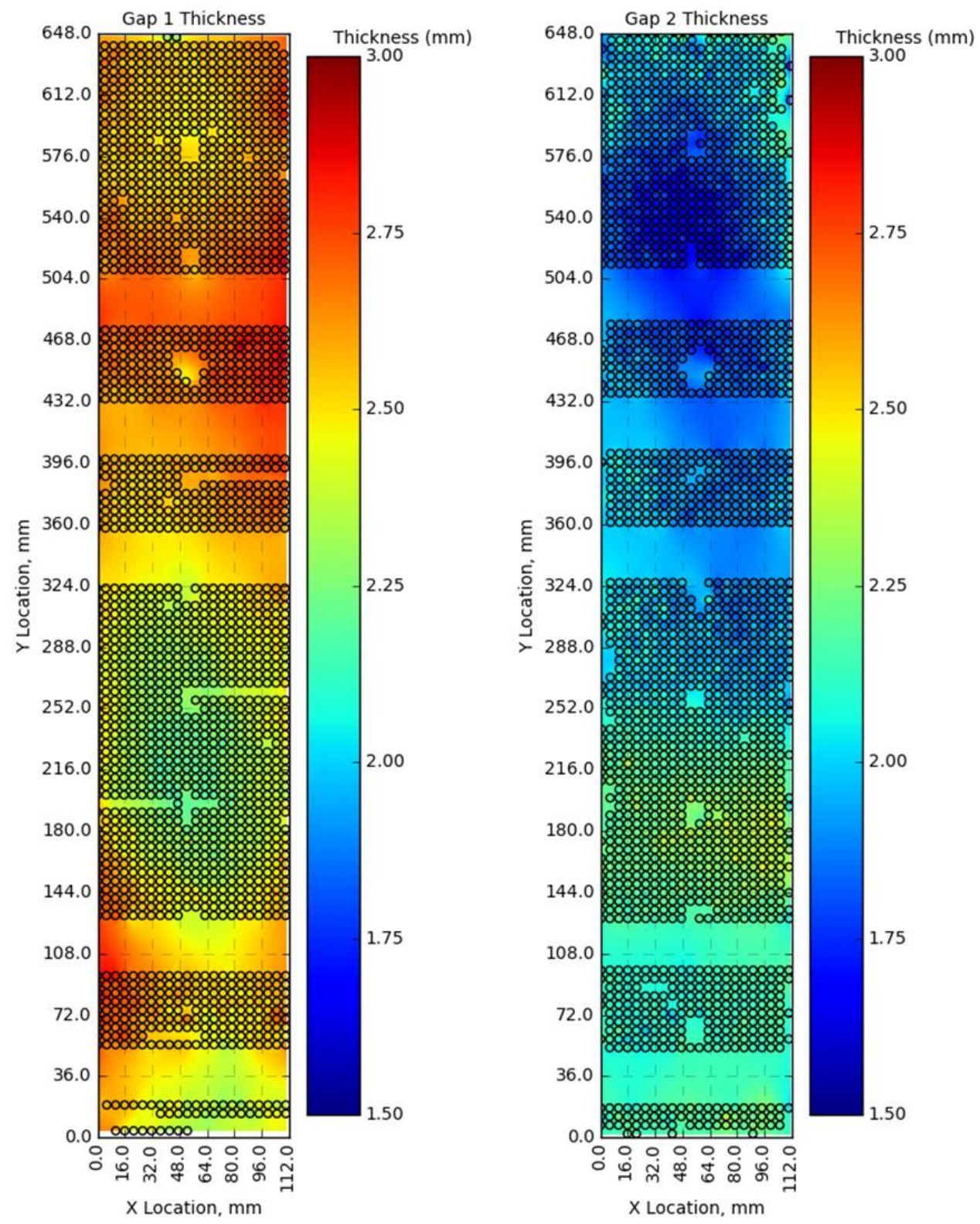

Figure 5.4. High-density channel thickness mapping

Table 5.2. Channel mapping summary data

\begin{tabular}{ccccc}
\hline & Ideal $(\mathrm{mm})$ & $\begin{array}{c}\text { Minimum } \\
(\mathrm{mm})\end{array}$ & Mean $(\mathrm{mm})$ & $\begin{array}{c}\text { Maximum } \\
(\mathrm{mm})\end{array}$ \\
\hline Channel 1 & 2.540 & 2.113 & 2.512 & 2.954 \\
\hline Channel 2 & 2.032 & 1.570 & 2.015 & 2.509 \\
\hline
\end{tabular}


The high-resolution channel mapping will be used in numeric models in Chapter 11. This will allow accurate representation of the fluid channel geometry in numeric simulations.

\section{2: Flow Testing Notes}

During testing, it was discovered that Pressure Transducer 8 (located $518.2 \mathrm{~mm}(20.4$ inches) from the plate trailing edge) was not providing an output signal. Efforts to troubleshoot and repair the transducer failed. Therefore, all data from that transducer has been discarded for this analysis. The eight remaining pressure transducers operated as expected.

When analyzing the pressure data, it was noted that the pressure transducers frequently recorded a negative pressure signal (i.e., the low side of the transducer was seeing higher pressure). Since the existing calibration did not consider negative pressure values, the calibrations were verified in this negative pressure range once testing was completed.

During the free edge (un-pinned) experiments with the lasers $290.5 \mathrm{~mm}$ (11.4 inches) from the plate trailing edge, there was a significant loss of data from one of the lasers. During the six lowest flow rates, the laser monitoring the larger (ideally $2.540 \mathrm{~mm}$ ) channel recorded a channel gap of $0 \mathrm{~mm}$. The specific cause of this error is unknown, however it is likely that the laser was detecting the Plexi-glass/water interface rather than the surface of the plate (see Figure 3.3). While this is unusual, it would account for the abnormal data. The affected data was discarded for this analysis. The laser performed as 
expected during the five higher flow rates and Laser B (monitors the ideally $2.032 \mathrm{~mm}$ channel) experienced no significant loss of data.

As discussed previously, the average velocity in the test section is determined using Equation 3.1. When using this equation, the mass flow rate (m) is determined by the flow meter during the experiment. The total flow cross-sectional area (A) is found in Table 5.1, where it is listed as $505.88 \mathrm{~mm}^{2}\left(0.7841 \mathrm{in}^{2}\right)$. The final parameter, the density of water $(\rho)$ is set as $998 \mathrm{~kg} / \mathrm{m}^{3}$. Using this method, mass flow rate data presented here can easily be converted to an average channel velocity. Using the channel mapping, the measured mass flow rates are converted to average fluid channel velocities in Table 5.3 for the free (un-pinned) experiments, and Table 5.5 for the combed (pinned) experiments.

Overall the experiments were successful, with only minor complications. Aside from the factors outlined in this section, there are no known issues which may have an adverse impact on the results.

\section{3: Free-Edge Experiments}

\subsection{1: Flow Rates and Velocities}

Using the methodology described in section 4.4, the measured flow rates and corresponding calculated average channel velocities for the Free (Un-Pinned) edge experiments are provided in Table 5.3. Note that the average channel velocities assume the plexiglass is rigid and does not bow outward as the flow rate and pressure increases. 
Table 5.3. Measured flow rates and calculated average velocities for free edge experiments

\begin{tabular}{|c|c|c|c|c|c|c|c|c|c|c|c|c|c|}
\hline \multicolumn{14}{|c|}{ Laser Position } \\
\hline \multicolumn{2}{|c|}{$\begin{array}{l}\text { A - 0.500" } \\
\mathrm{kg} / \mathrm{s} \quad \mathrm{m} / \mathrm{s}\end{array}$} & \multicolumn{2}{|c|}{$\begin{array}{l}\text { B - 6.000" } \\
\mathrm{kg} / \mathrm{s} \quad \mathrm{m} / \mathrm{s}\end{array}$} & \multicolumn{2}{|c|}{$\begin{array}{l}\mathrm{C}-8.925^{\prime \prime} \\
\mathrm{kg} / \mathrm{s} \quad \mathrm{m} / \mathrm{s}\end{array}$} & \multicolumn{2}{|c|}{$\begin{array}{l}\text { D - 11.438" } \\
\mathrm{kg} / \mathrm{s} \quad \mathrm{m} / \mathrm{s}\end{array}$} & \multicolumn{2}{|c|}{$\begin{array}{l}\text { E - 18.675" } \\
\mathrm{kg} / \mathrm{s} \quad \mathrm{m} / \mathrm{s}\end{array}$} & \multicolumn{2}{|c|}{$\begin{array}{l}\text { F - 21.675" } \\
\mathrm{kg} / \mathrm{s} \quad \mathrm{m} / \mathrm{s}\end{array}$} & \multicolumn{2}{|c|}{$\begin{array}{l}\text { G - 25.000' } \\
\mathrm{kg} / \mathrm{s} \quad \mathrm{m} / \mathrm{s}\end{array}$} \\
\hline 1.048 & 2.076 & 1.044 & 2.068 & 1.034 & 2.049 & 1.042 & 2.064 & 1.039 & 2.059 & 1.027 & 2.034 & 1.060 & 2.100 \\
\hline 1.414 & 2.802 & 1.392 & 2.756 & 1.376 & 2.726 & 1.419 & 2.811 & 1.357 & 2.688 & 1.378 & 2.729 & 1.458 & 2.887 \\
\hline 1.807 & 3.580 & 1.770 & 3.505 & 1.671 & 3.310 & 1.699 & 3.366 & 1.730 & 3.426 & 1.721 & 3.409 & 1.815 & 3.595 \\
\hline 2.350 & 4.655 & 2.283 & 4.521 & 2.244 & 4.445 & 2.375 & 4.705 & 2.228 & 4.413 & 2.321 & 4.596 & 2.289 & 4.534 \\
\hline 2.635 & 5.219 & 2.574 & 5.098 & 2.509 & 4.970 & 2.626 & 5.200 & 2.558 & 5.066 & 2.634 & 5.218 & 2.638 & 5.224 \\
\hline 2.846 & 5.637 & 2.721 & 5.389 & 2.739 & 5.425 & 2.796 & 5.537 & 2.745 & 5.438 & 2.755 & 5.458 & 2.728 & 5.403 \\
\hline 2.926 & 5.796 & 2.853 & 5.651 & 2.827 & 5.599 & 2.781 & 5.509 & 2.872 & 5.689 & 2.912 & 5.768 & 2.817 & 5.580 \\
\hline 2.917 & 5.778 & 2.938 & 5.819 & 2.960 & 5.862 & 2.953 & 5.848 & 3.036 & 6.013 & 3.015 & 5.971 & 2.864 & 5.674 \\
\hline 3.454 & 6.842 & 3.473 & 6.880 & 3.403 & 6.740 & 3.404 & 6.742 & 3.419 & 6.772 & 3.431 & 6.796 & 3.411 & 6.757 \\
\hline 3.843 & 7.612 & 3.961 & 7.846 & 3.973 & 7.870 & 3.849 & 7.624 & 3.929 & 7.782 & 3.871 & 7.668 & 3.945 & 7.813 \\
\hline 4.325 & 8.567 & 4.435 & 8.784 & 4.300 & 8.518 & 4.306 & 8.528 & 4.347 & 8.610 & 4.285 & 8.488 & 4.363 & 8.642 \\
\hline
\end{tabular}

\subsection{2: Channel Gap Results}

Figure 5.5 through Figure 5.11 provide the measured channel gap and corresponding change in channel gap thickness at the seven axial laser positions (see Figure 4.3 and Table 4.3 for the measurement locations corresponding to each pair of plots). The change in channel gap is relative to an initial channel thickness, which was measured before each flow test. The change in channel gap can reasonably be interpreted as plate deflection. See Appendix B for the initial gap values. 

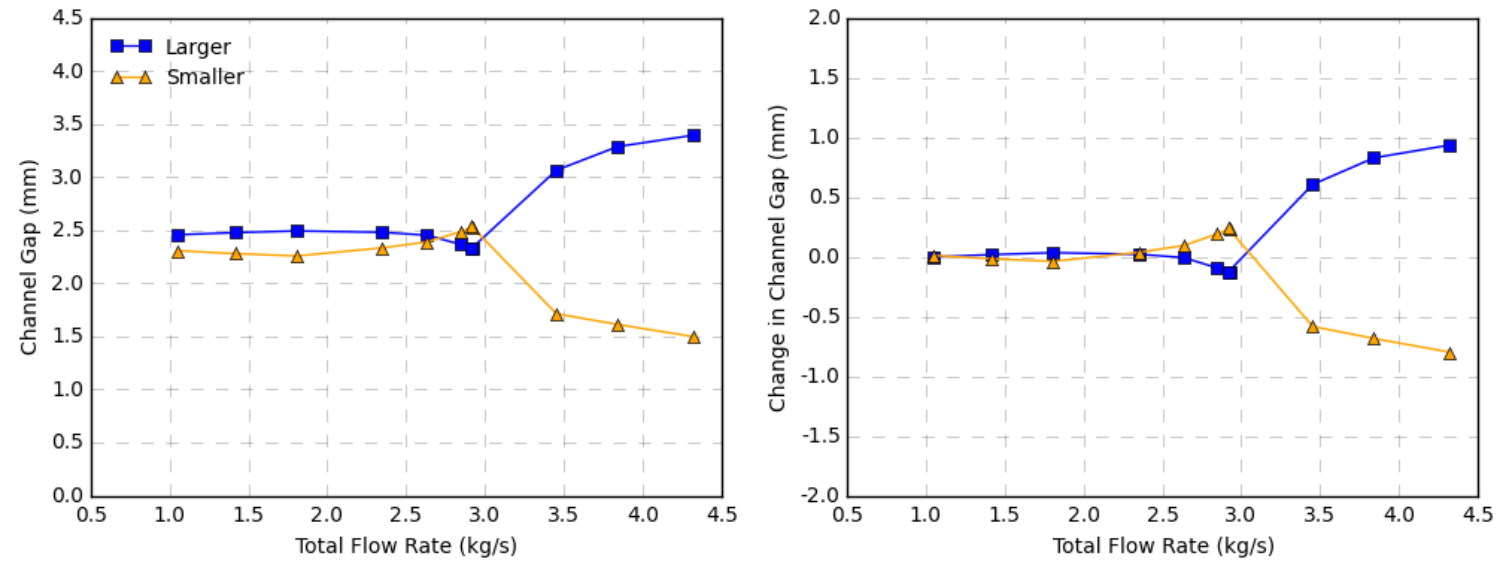

Figure 5.5. Channel gap and deflection at location $A-12.7 \mathrm{~mm}$ from trailing edge with free edges
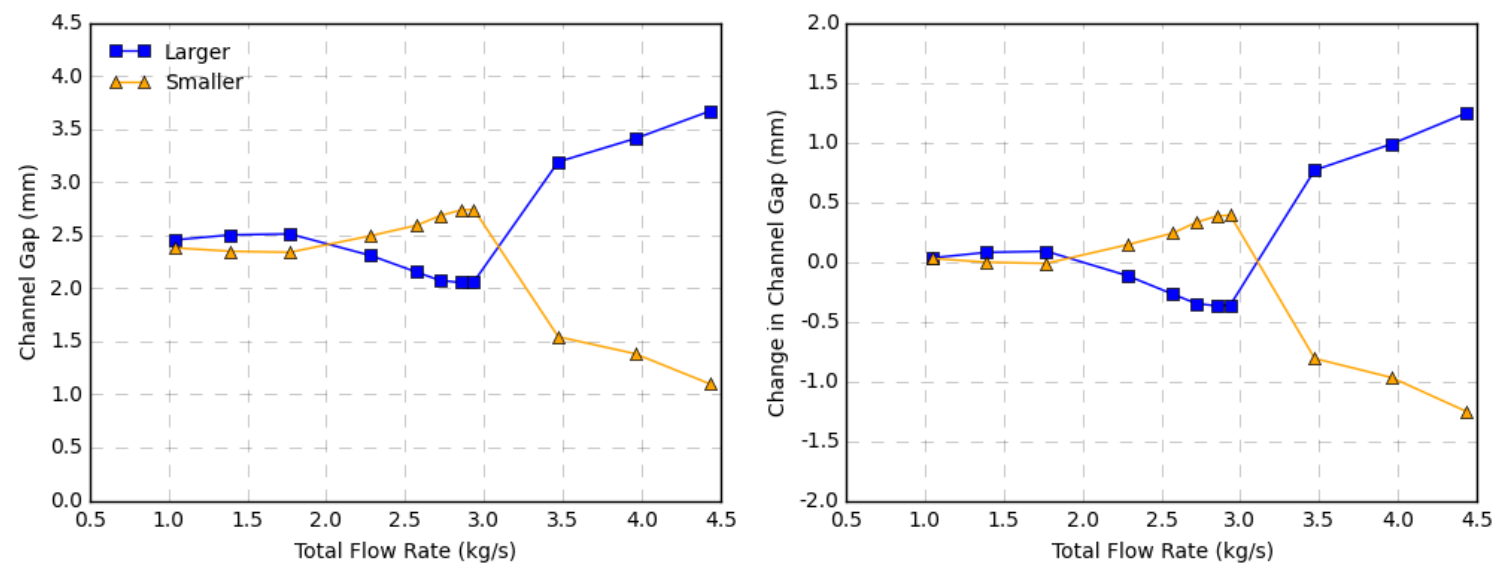

Figure 5.6. Channel gap and deflection at location $B-152.4 \mathrm{~mm}$ from trailing edge with free edges
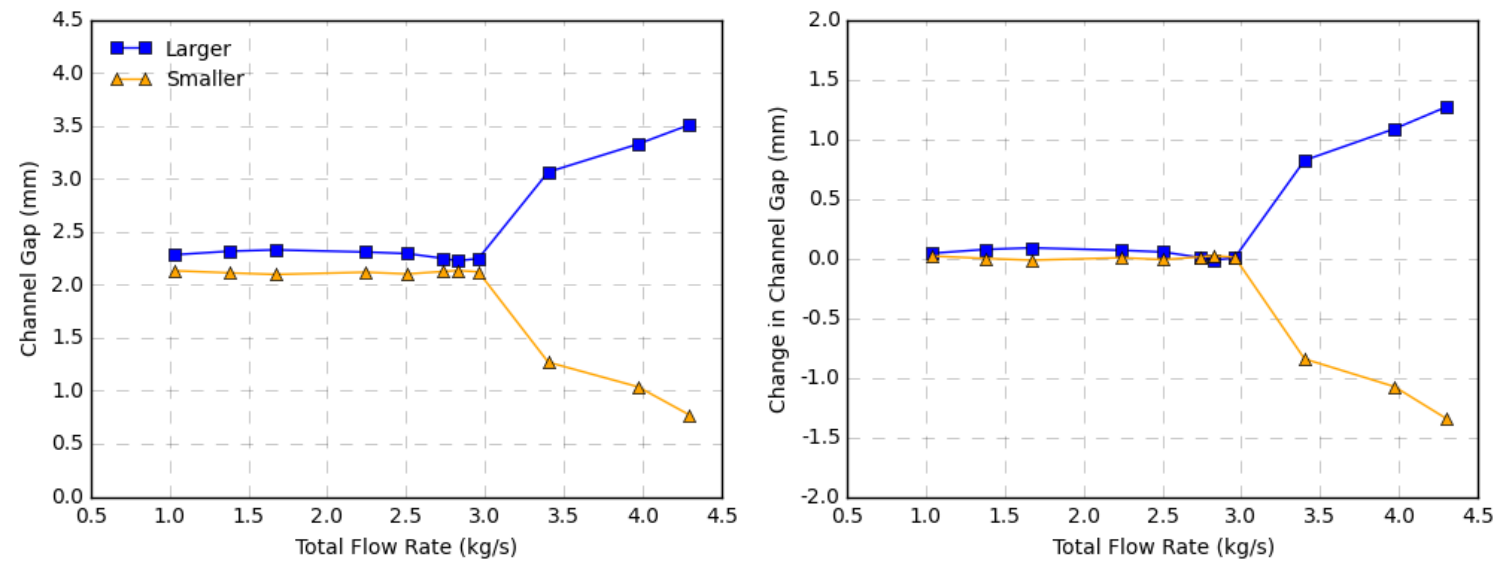

Figure 5.7. Channel gap and deflection at location $\mathrm{C}-226.7 \mathrm{~mm}$ from trailing edge with free edges 

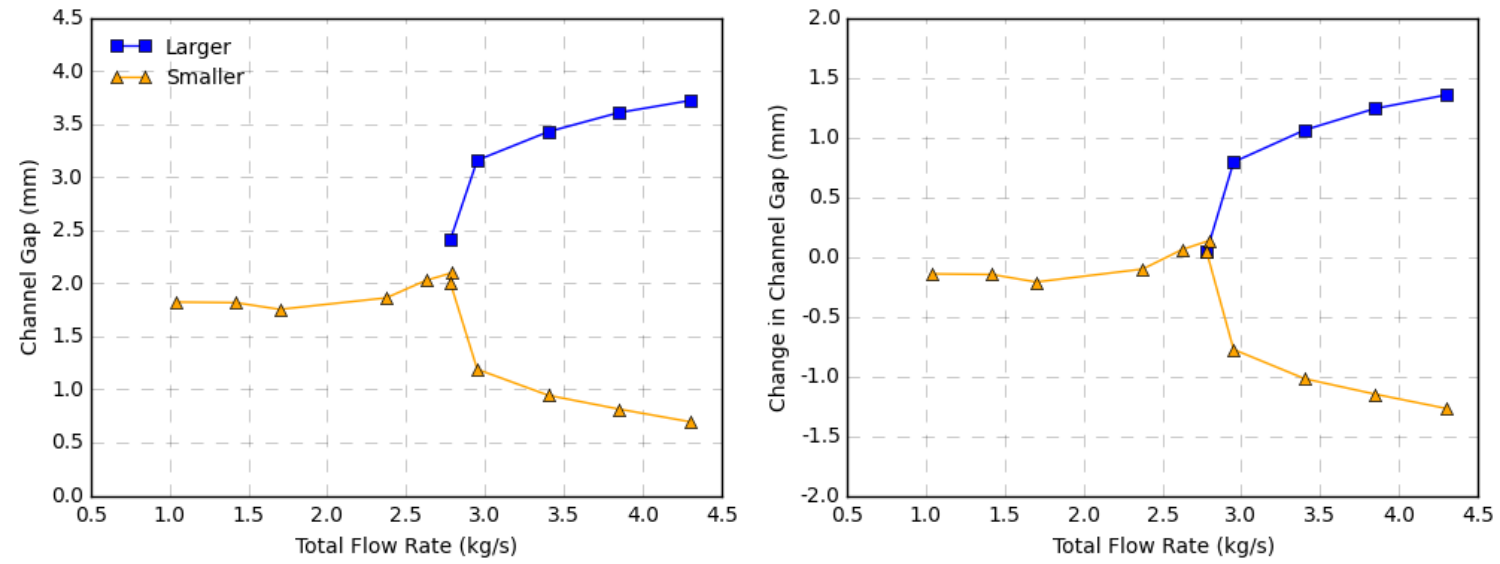

Figure 5.8. Channel gap and deflection at location $D-290.5 \mathrm{~mm}$ from trailing edge with free edges
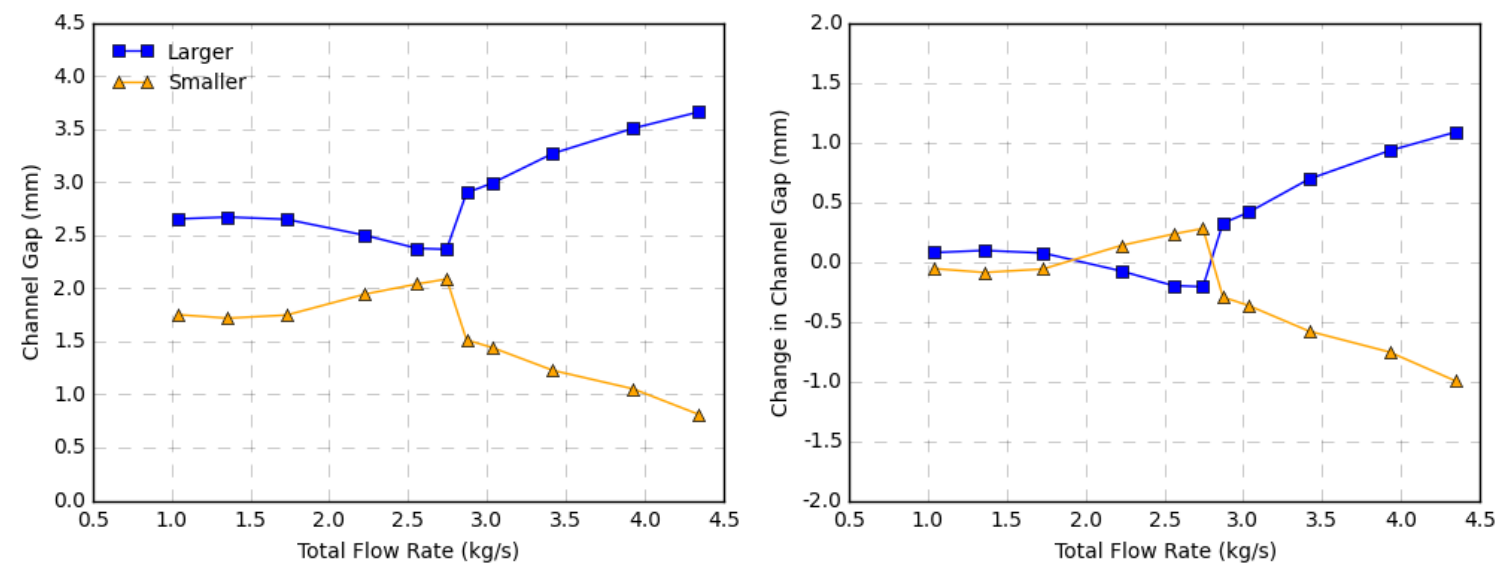

Figure 5.9. Channel gap and deflection at location $E-474.3 \mathrm{~mm}$ from trailing edge with free edges
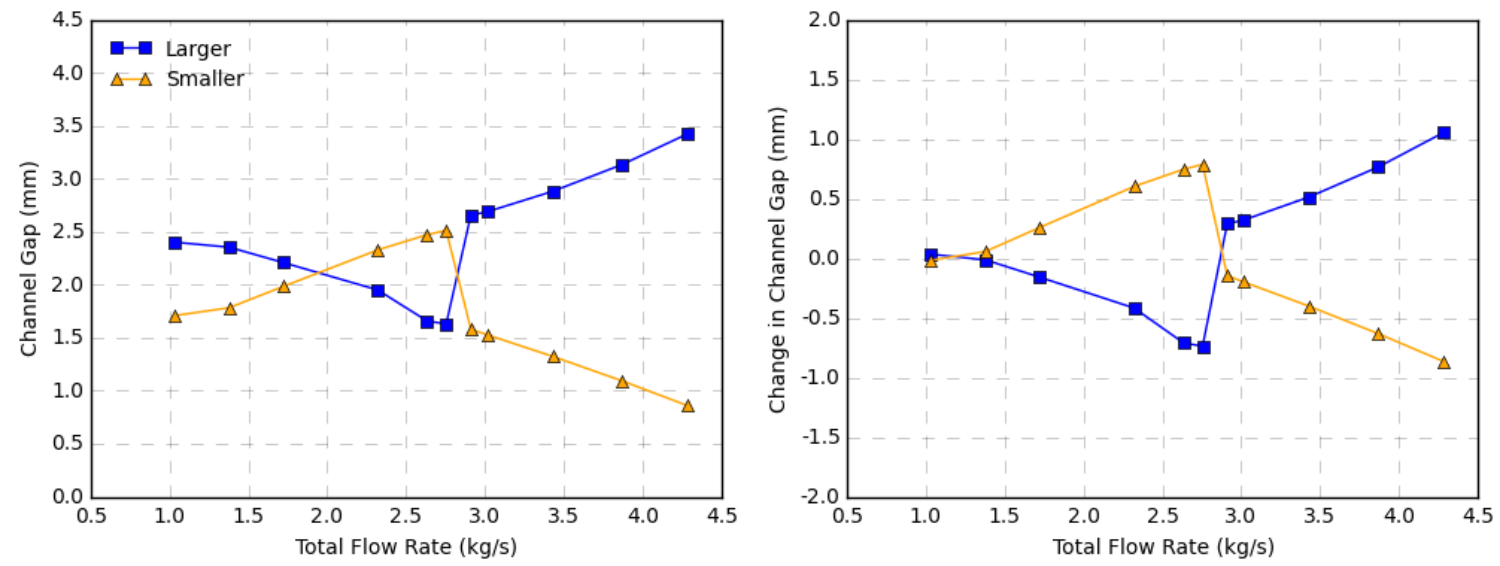

Figure 5.10. Channel gap and deflection at location $F-550.5 \mathrm{~mm}$ from trailing edge with free edges 

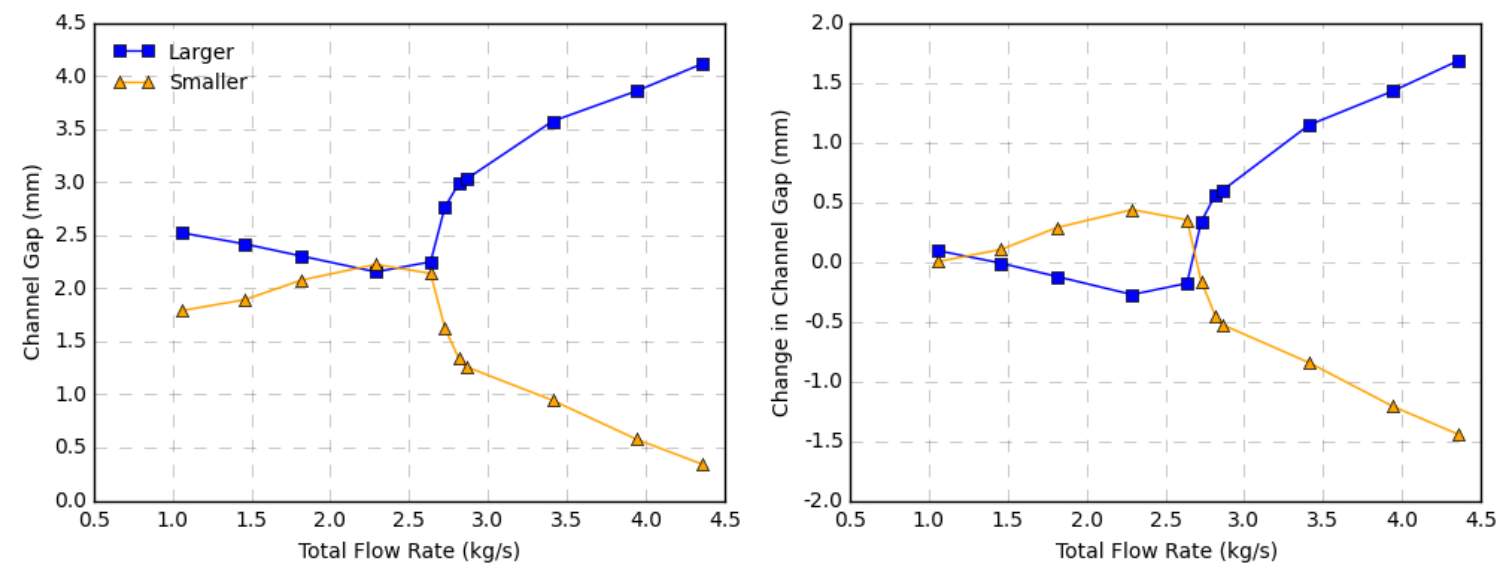

Figure 5.11. Channel gap and deflection at location $G-635.0 \mathrm{~mm}$ from trailing edge with free edges Using data for the change in the smaller channel thickness from the preceding figures, an estimation of the plate profile at various velocities is shown in Figure 5.12. Note that since the actual flow rates (see Table 5.3) varied somewhat among the different laser positions, an average of the measured flow rates was used for the legend in Figure 5.12. Additionally, a review of the preceding figures shows that the plate snaps from one channel to the other in every flow test. This snap occurred at a slightly different flow rate each time. This resulted in three plate position configurations:

1. A stable, 'pre-snap' deflection at flow rates below $2.64 \mathrm{~kg} / \mathrm{s}$.

2. A transition region from 2.64 to $3.47 \mathrm{~kg} / \mathrm{s}$.

3. A stable, 'post-snap' deflection at velocities above $3.47 \mathrm{~kg} / \mathrm{s}$.

In Figure 5.12, three data sets in the transition range (representing $2.761 \mathrm{~kg} / \mathrm{s}, 2.855 \mathrm{~kg} / \mathrm{s}$ and $2.955 \mathrm{~kg} / \mathrm{s}$ ) have been omitted since they include measurements from both before and after the plate snap. Since the data at each axial location is collected independently (a new velocity sweep is completed after repositioning the lasers), the data sets in the velocity transition range may include both pre-snap and post-snap measurements. 


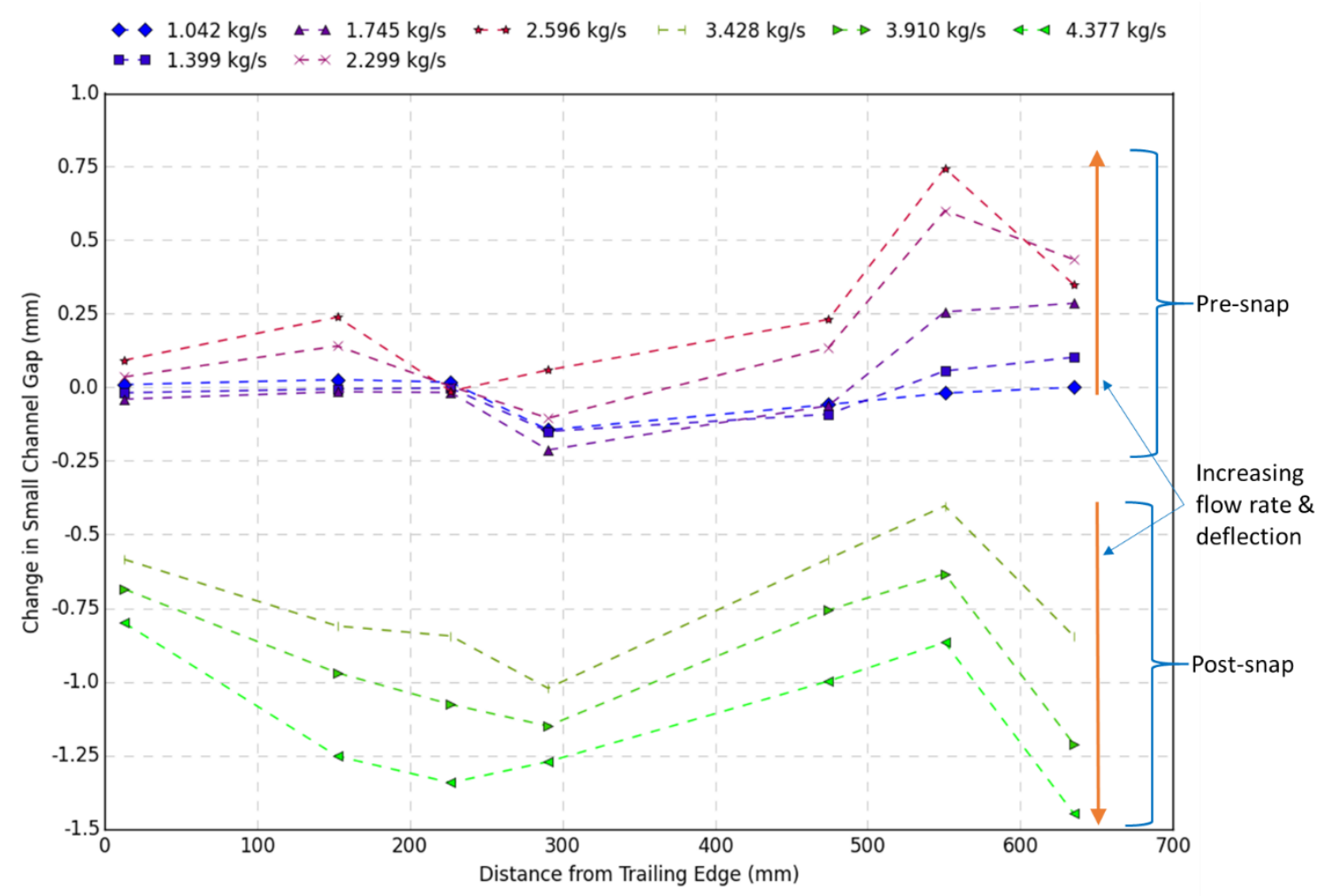

Figure 5.12. Plate profiles at various flow rates with free edges

From examining Figure 5.12, two distinct plate profiles (pre-snap and post-snap) can be identified. The magnitude of the deflection in both profiles increases with flow rate. It is also notable that the rate of increase in the deflection as the plate approaches the 'snap velocity' drops.

\subsection{3: Pressure Results}

In order to acquire laser data at all seven axial locations, the experiment was essentially repeated seven times with the lasers in a different location each time. Since the pressure taps remain in the same location for each experiment, there are seven repeated trials of the same pressure data. 
Figure 5.13 through Figure 5.20 each represents one of the eight working pressure transducers (recall that Pressure Transducer 8 was inoperative). Each figure contains data from all seven trials $(A-G)$ for the specified pressure transducer. The trials are identified in the legend based on the location the lasers happened to be at for that test, although the laser position should have no bearing on pressure results. The pressure difference is defined as $\mathrm{P}_{\text {Smaller Channel }}-\mathrm{P}_{\text {Larger Channel. }}$

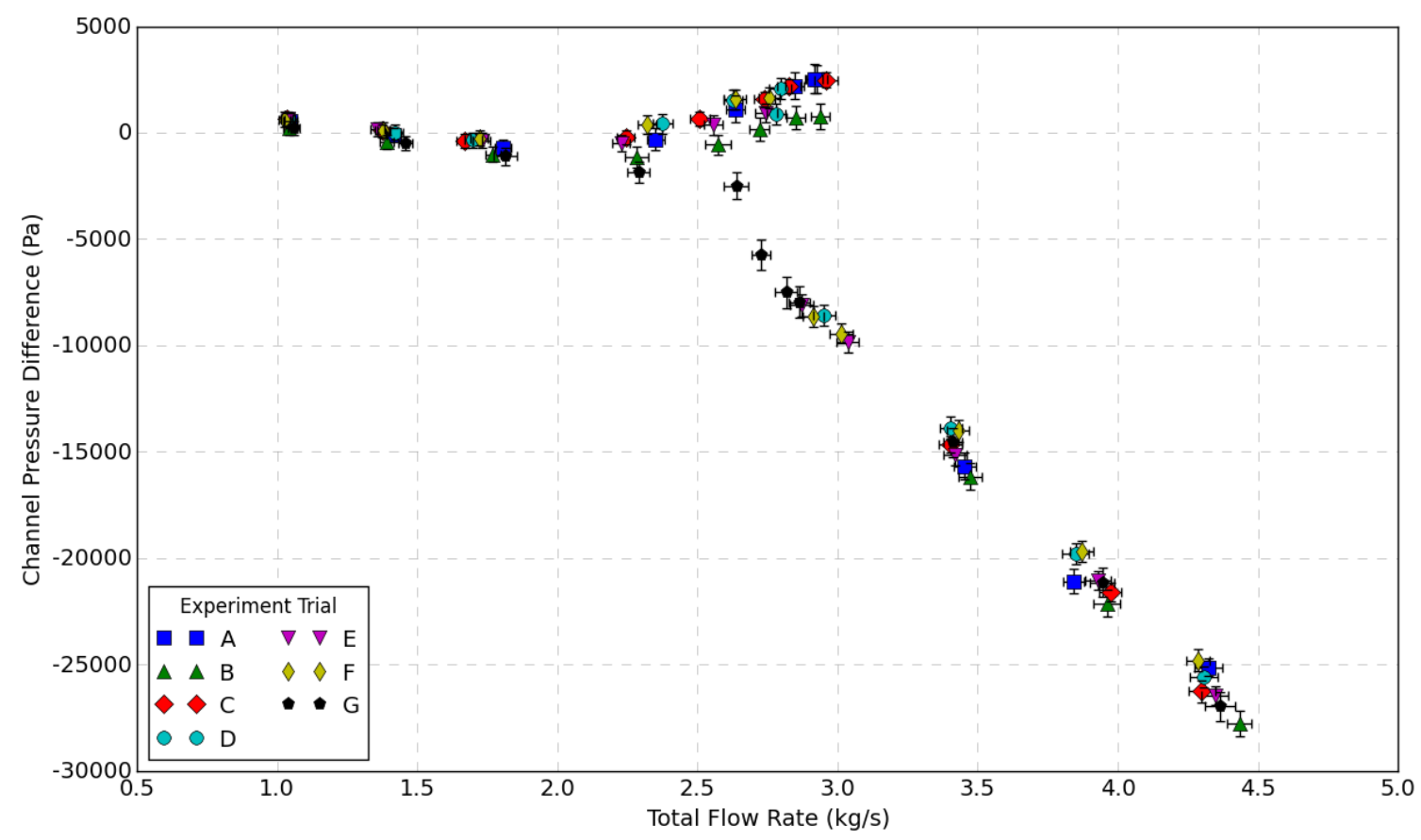

Figure 5.13. Channel pressure difference in free edge experiments, $64.77 \mathrm{~mm}(2.55 ")$ from plate trailing edge (PT-1) 


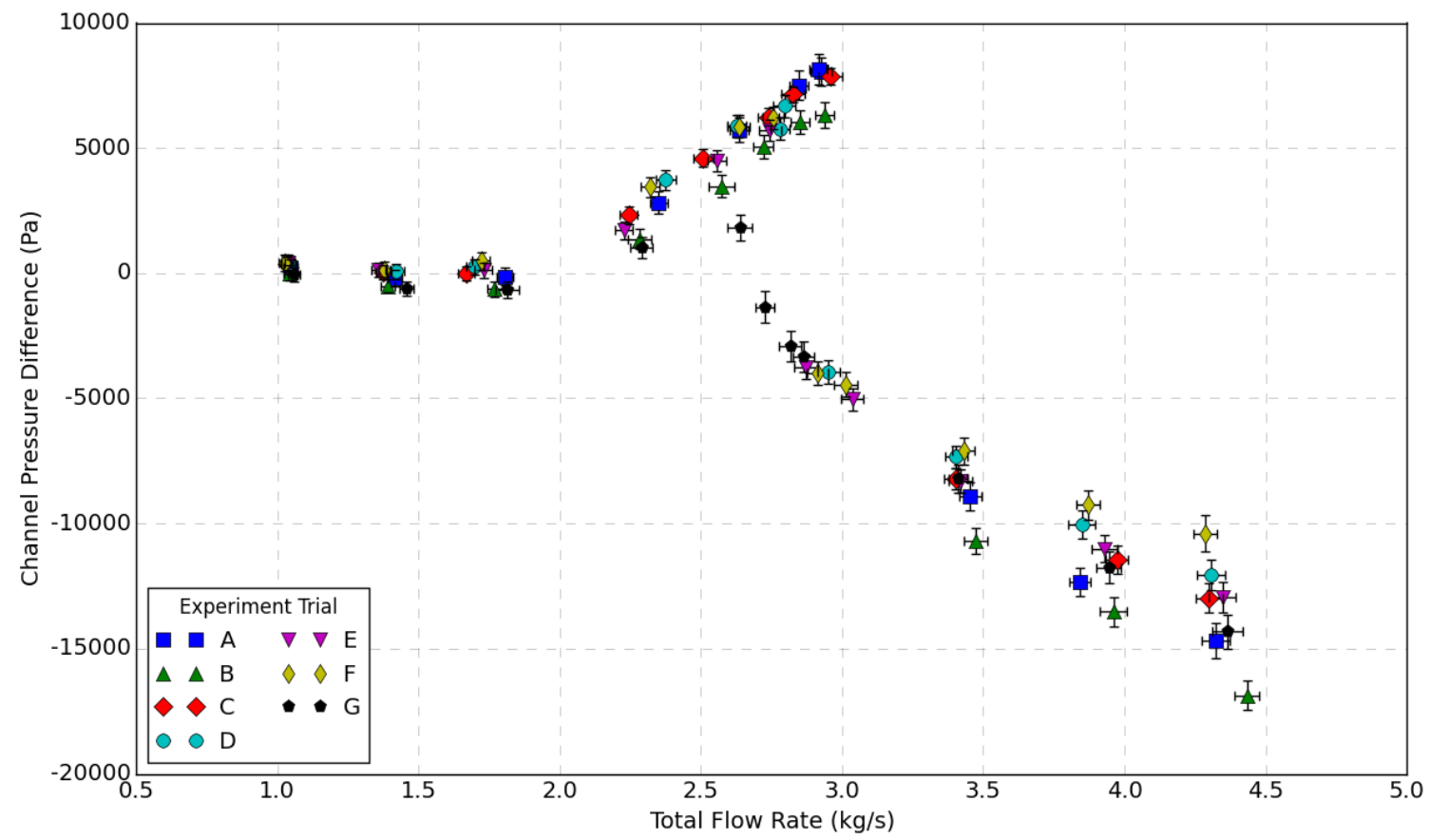

Figure 5.14. Channel pressure difference in free edge experiments, $129.54 \mathrm{~mm}\left(5.10^{\prime \prime}\right)$ from plate trailing edge (PT-2)

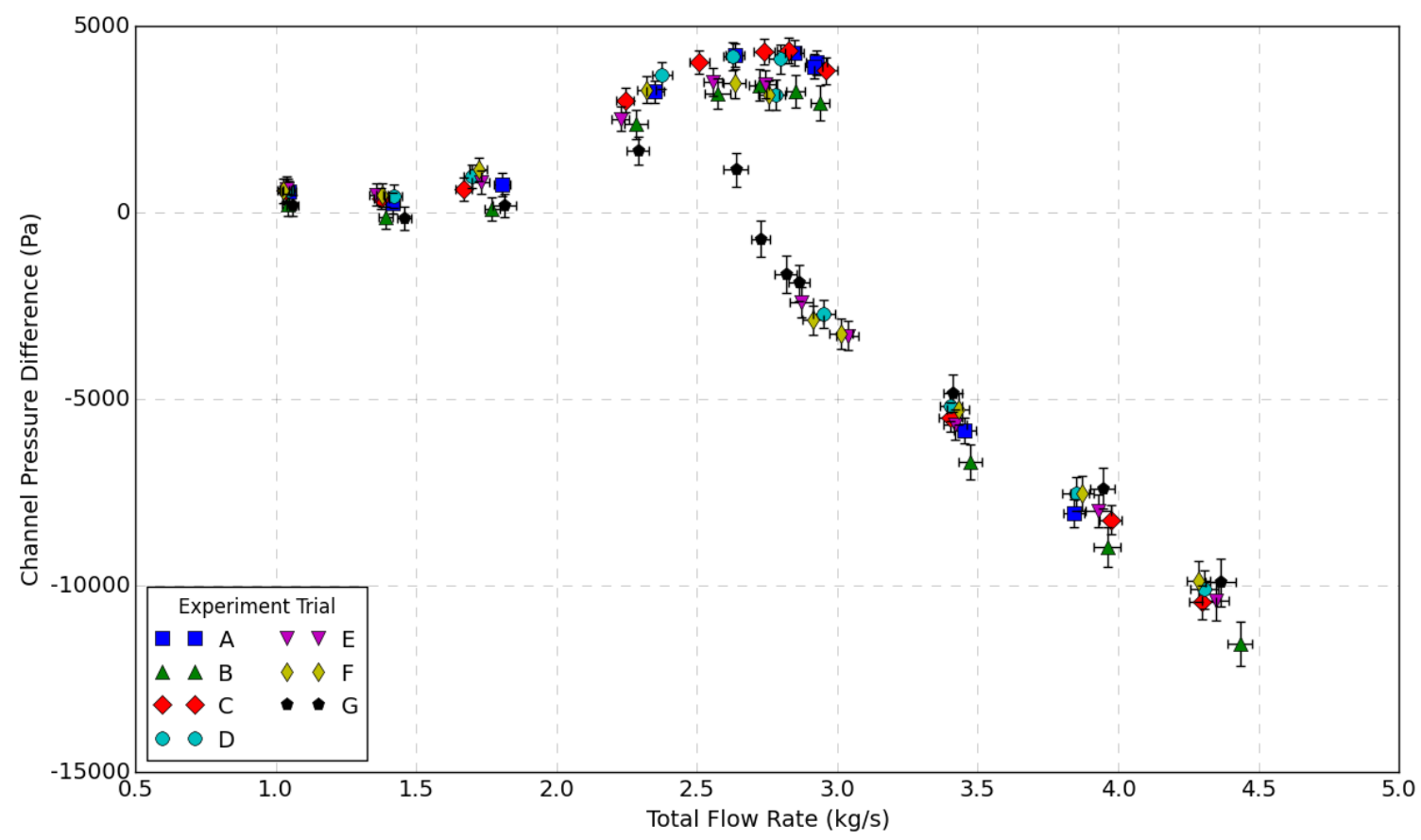

Figure 5.15. Channel pressure difference in free edge experiments, $194.31 \mathrm{~mm}(7.65 ")$ from plate trailing edge (PT-3) 


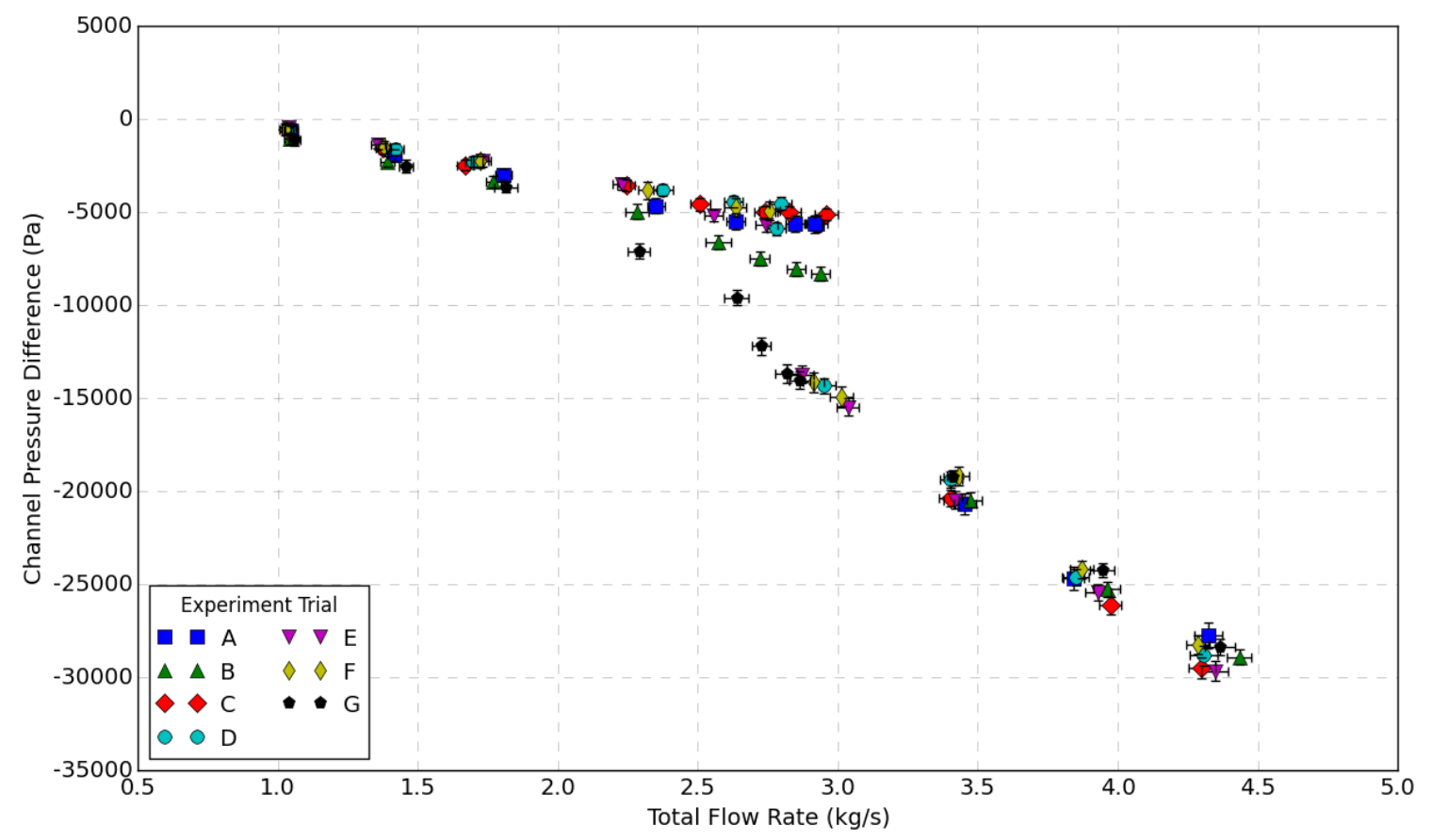

Figure 5.16. Channel pressure difference in free edge experiments, $259.08 \mathrm{~mm}(10.20 ")$ from plate trailing edge (PT-4)

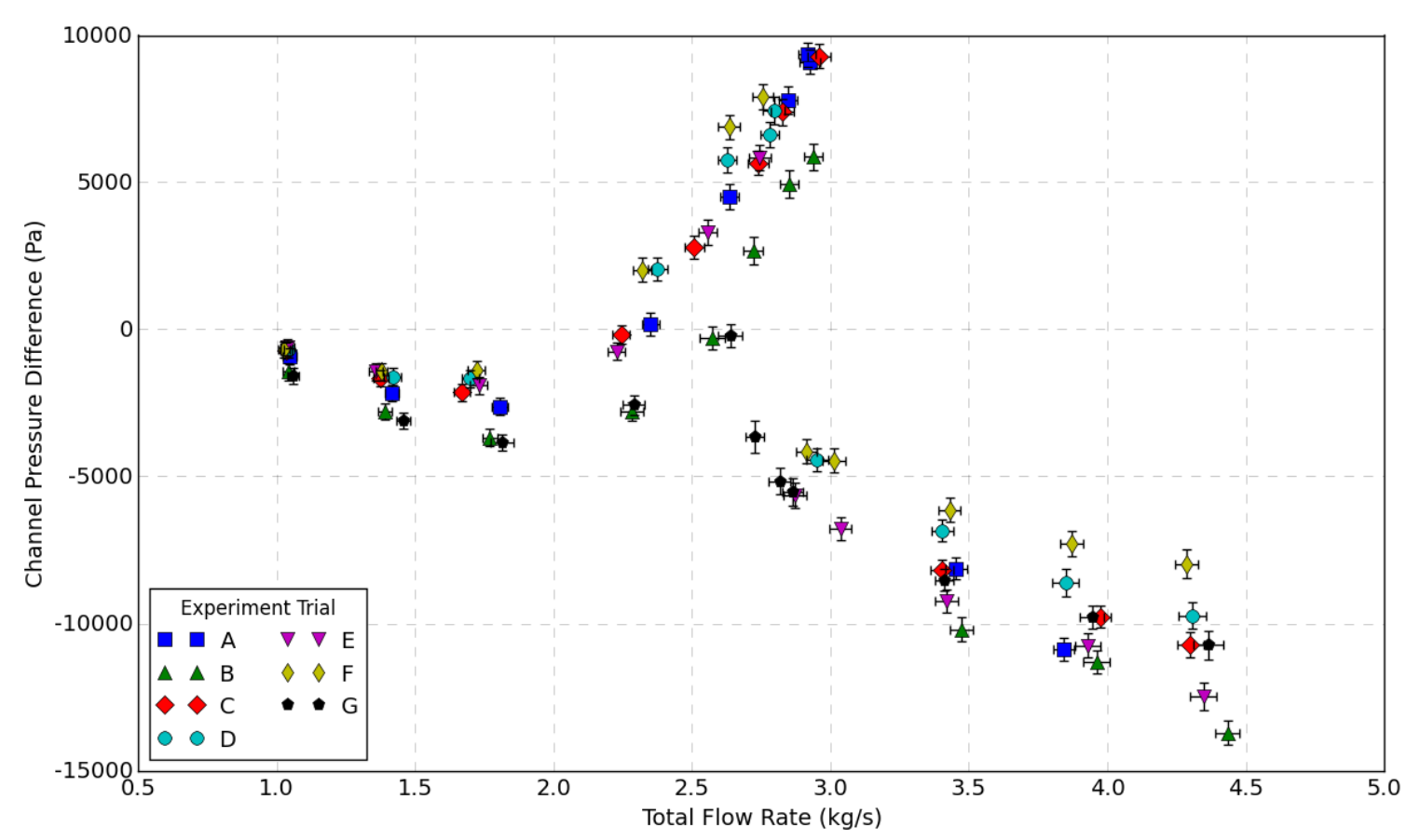

Figure 5.17. Channel pressure difference in free edge experiments, $323.85 \mathrm{~mm}(12.75 ")$ from plate trailing edge (PT-5) 


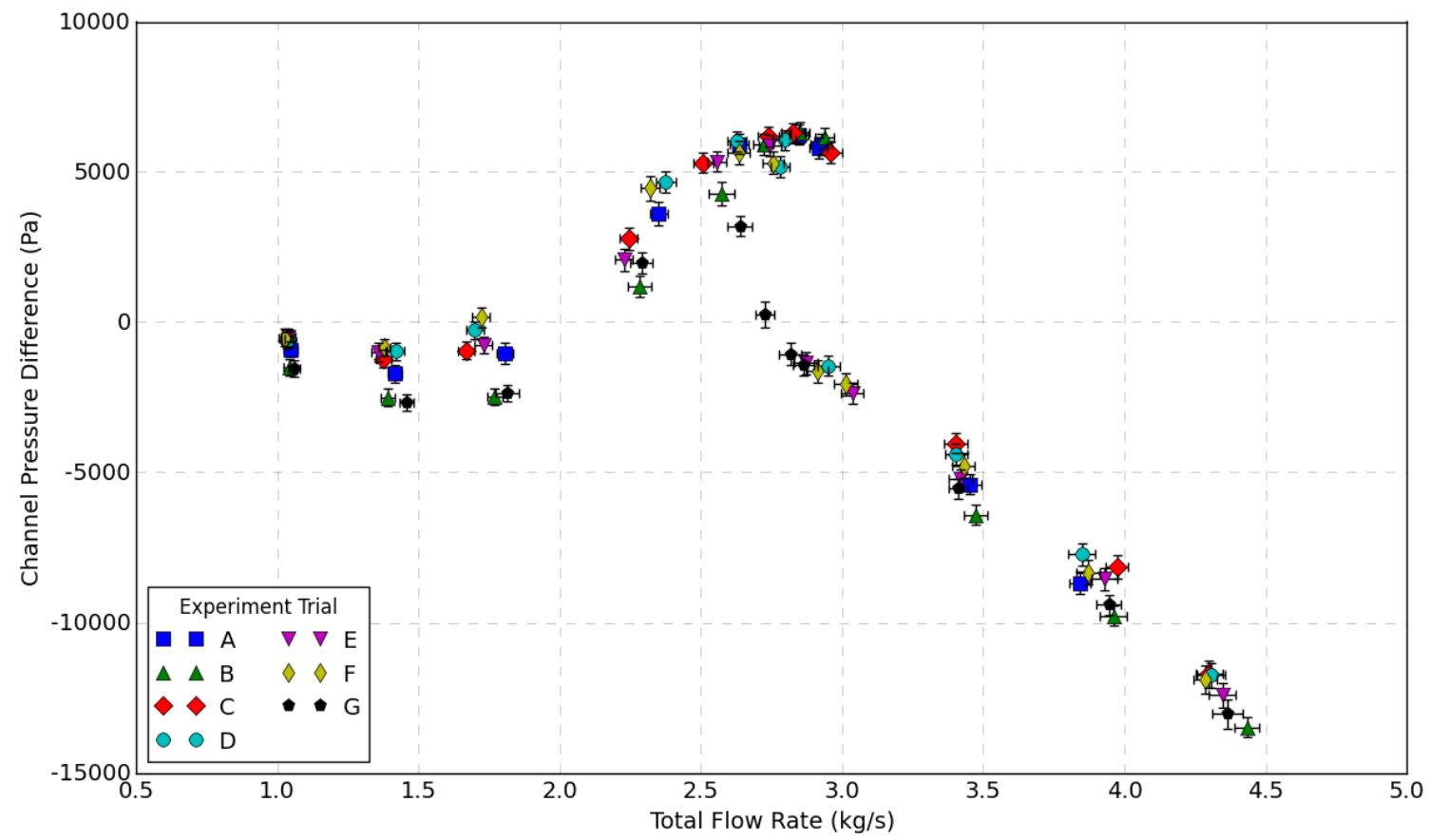

Figure 5.18. Channel pressure difference in free edge experiments, $388.62 \mathrm{~mm}(15.30 ")$ from plate trailing edge (PT-6)

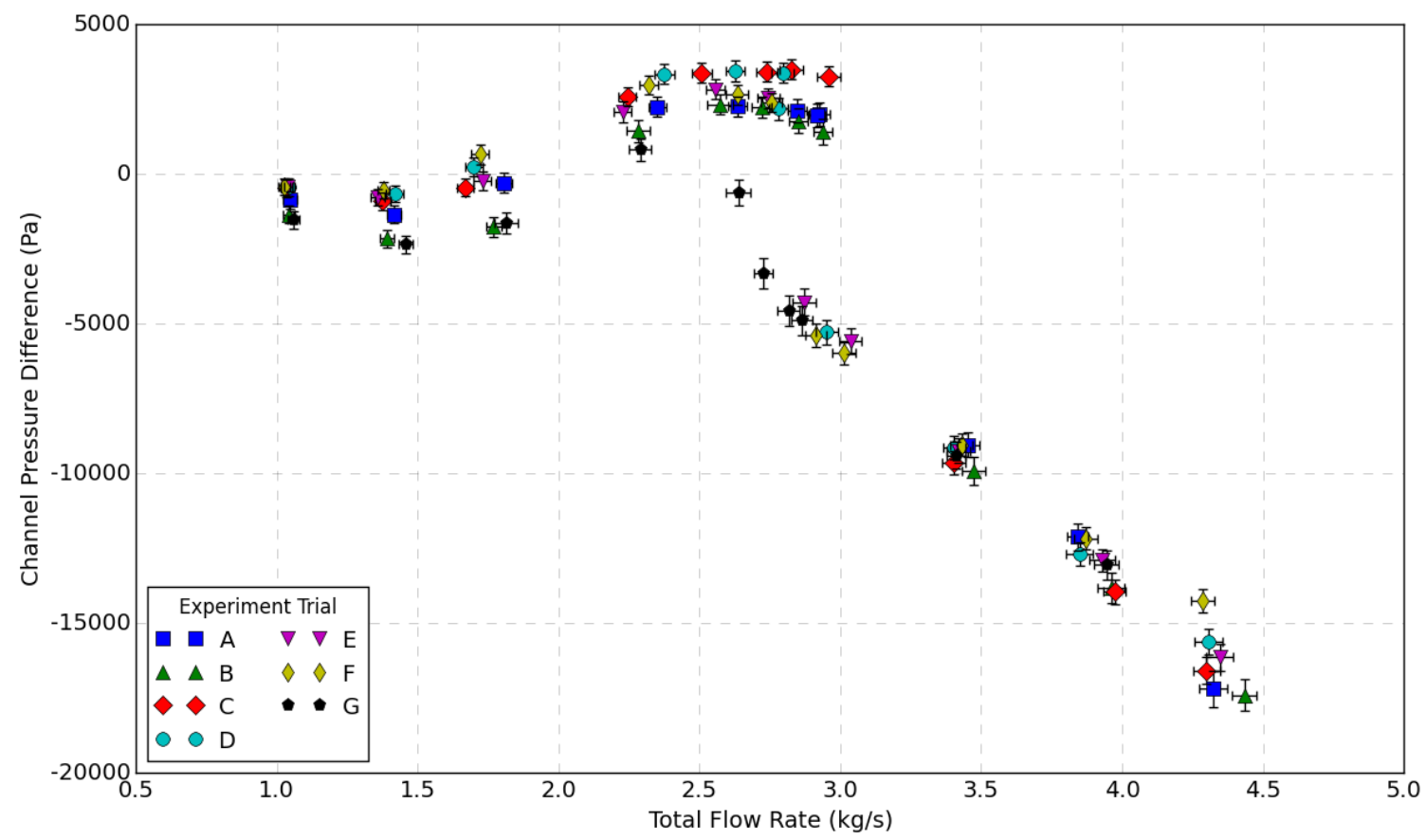

Figure 5.19. Channel pressure difference in free edge experiments, $453.39 \mathrm{~mm}(17.85 ")$ from plate trailing edge (PT-7) 


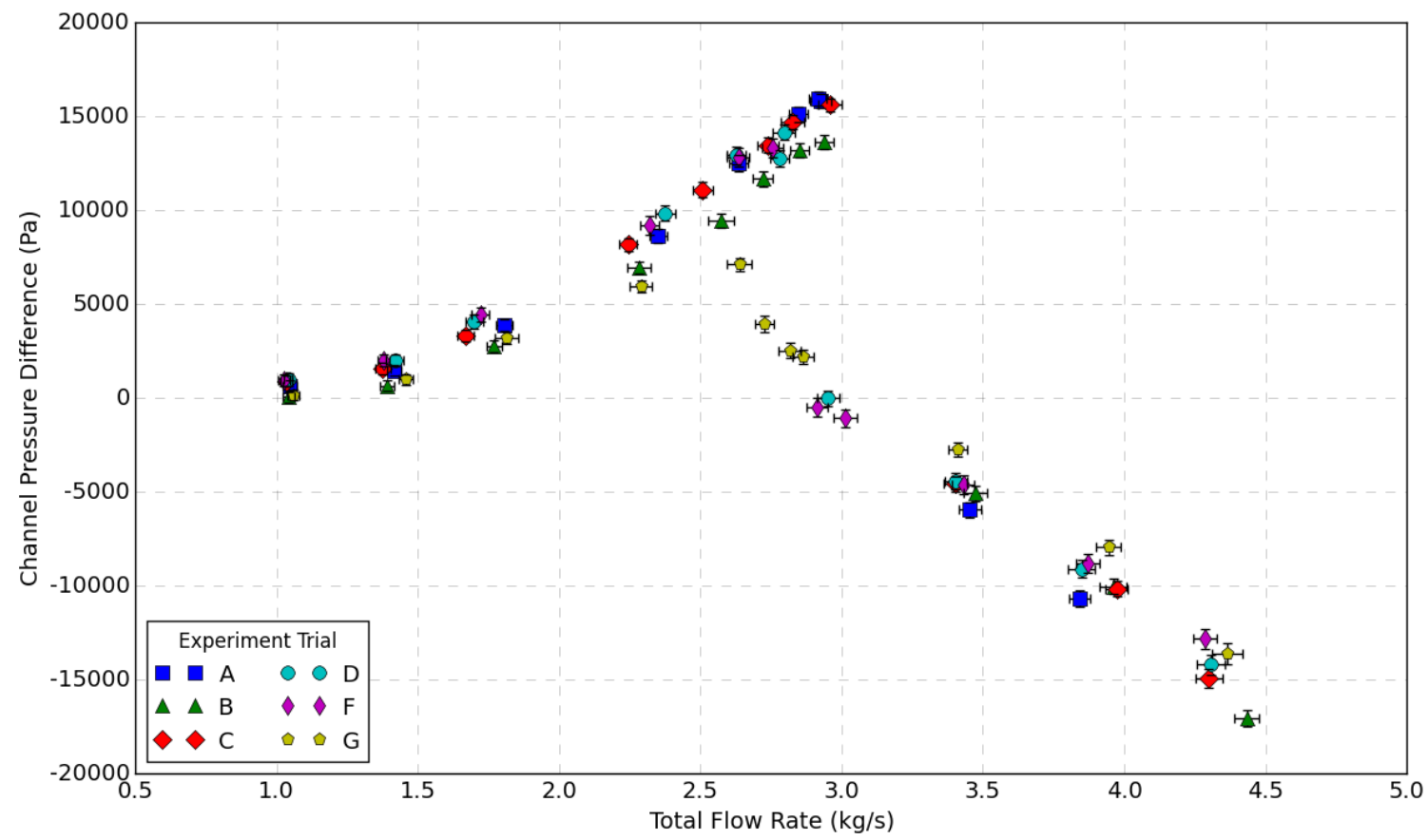

Figure 5.20. Channel pressure difference in free edge experiments, $582.93 \mathrm{~mm}(22.95 ")$ from plate trailing edge (PT-9)

Much like the channel gap and deflection plots, there is an apparent shift in the pressure when the plate snaps from one channel to the other. As was noted earlier, the flow rate at which the plate snaps varies somewhat from trial to trial. This variation is apparent in the pressure data between approximately 2.5 and $3 \mathrm{~kg} / \mathrm{s}$, with trials $\mathrm{A}, \mathrm{B}$, and $\mathrm{C}$ tending to snap at higher flow rates, and trial $\mathrm{G}$ tending to snap at a lower flow rate. The other trials appear to be somewhere in between.

\subsection{4: Snap Velocity Variability}

For each flow test, channel gap data was collected from the lasers at a single axial location and pressure data was collected from the transducers at all eight axial locations. Recall that one flow test is comprised of a sweep over the entire flow rate range. Each flow test was repeated seven times, where the axial position of the laser heads was 
changed between each flow test in order to allow recording of channel gap data at seven axial locations. During each flow test, the plate was observed to deflect into the larger channel at lower flow rates before snapping into the smaller channel at higher flow rates. The exact flow rate at which the snap occurred varied between flow tests.

Comparison of the deflection data at each axial location (A-G) in Figure 5.5 through Figure 5.11 to the corresponding data from all eight pressure transducers in Figure 5.13 through Figure 5.20 yields an interesting conclusion. For a given flow test A-G, both lasers and all eight pressure transducers registered the plate snap at the same point during flow testing. This would seem to indicate that when the plate snapped from one channel to the other, the entire plate snapped at once, and not gradually as flow increased.

The lower and upper bounds for each of plate snap flow rates from the deflection plots are noted in Table 5.4, with the overall bounds for the snap flow rate highlighted. Using these bounds, we can infer the transition flow rate range over which the plate snap occurs. From Table 5.4, this range corresponds to $2.64 \mathrm{~kg} / \mathrm{s}$ through $3.47 \mathrm{~kg} / \mathrm{s}$. 
Table 5.4. Lower and upper bounds for snap flow rate during each flow test

\begin{tabular}{ccc}
\hline Flow Test (based on Laser Location) & Lower & Upper \\
\hline A & $2.92 \mathrm{~kg} / \mathrm{s}$ & $3.45 \mathrm{~kg} / \mathrm{s}$ \\
B & $2.94 \mathrm{~kg} / \mathrm{s}$ & $3.47 \mathrm{~kg} / \mathrm{s}$ \\
C & $2.96 \mathrm{~kg} / \mathrm{s}$ & $3.40 \mathrm{~kg} / \mathrm{s}$ \\
D & $2.78 \mathrm{~kg} / \mathrm{s}$ & $2.95 \mathrm{~kg} / \mathrm{s}$ \\
E & $2.75 \mathrm{~kg} / \mathrm{s}$ & $2.87 \mathrm{~kg} / \mathrm{s}$ \\
F & $2.76 \mathrm{~kg} / \mathrm{s}$ & $2.91 \mathrm{~kg} / \mathrm{s}$ \\
G & $2.64 \mathrm{~kg} / \mathrm{s}$ & $2.73 \mathrm{~kg} / \mathrm{s}$ \\
\hline
\end{tabular}

\subsection{5: Instantaneous Plate Snap}

On two occasions during flow testing, the plate snap occurred during data collection.

Since the data analysis relied on the plate assuming a steady state position during data collection, these two data sets were not used in the preceding analysis. However, the data was saved to allow for closer inspection of the snap behavior.

The first data set showing the plate snap occurred with the lasers located at axial location $\mathrm{E}(474.345 \mathrm{~mm}$ from the trailing edge) at a flow rate of $2.927 \mathrm{~kg} / \mathrm{s}$. In the following figures, a five second excerpt from the data with the snap included is presented. 


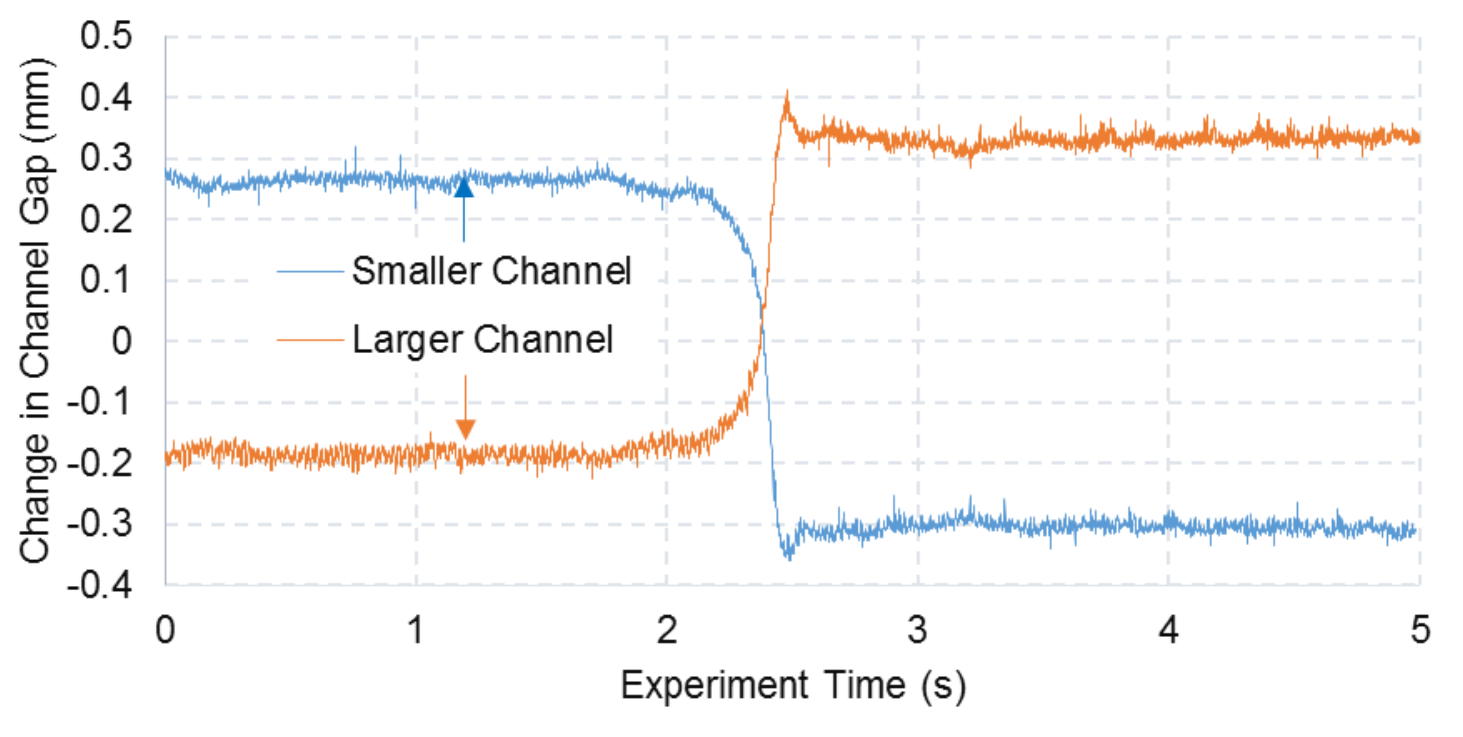

Figure 5.21. Change in channel gap during plate snap at axial location $E$ and flow rate of $2.927 \mathrm{~kg} / \mathrm{s}$.

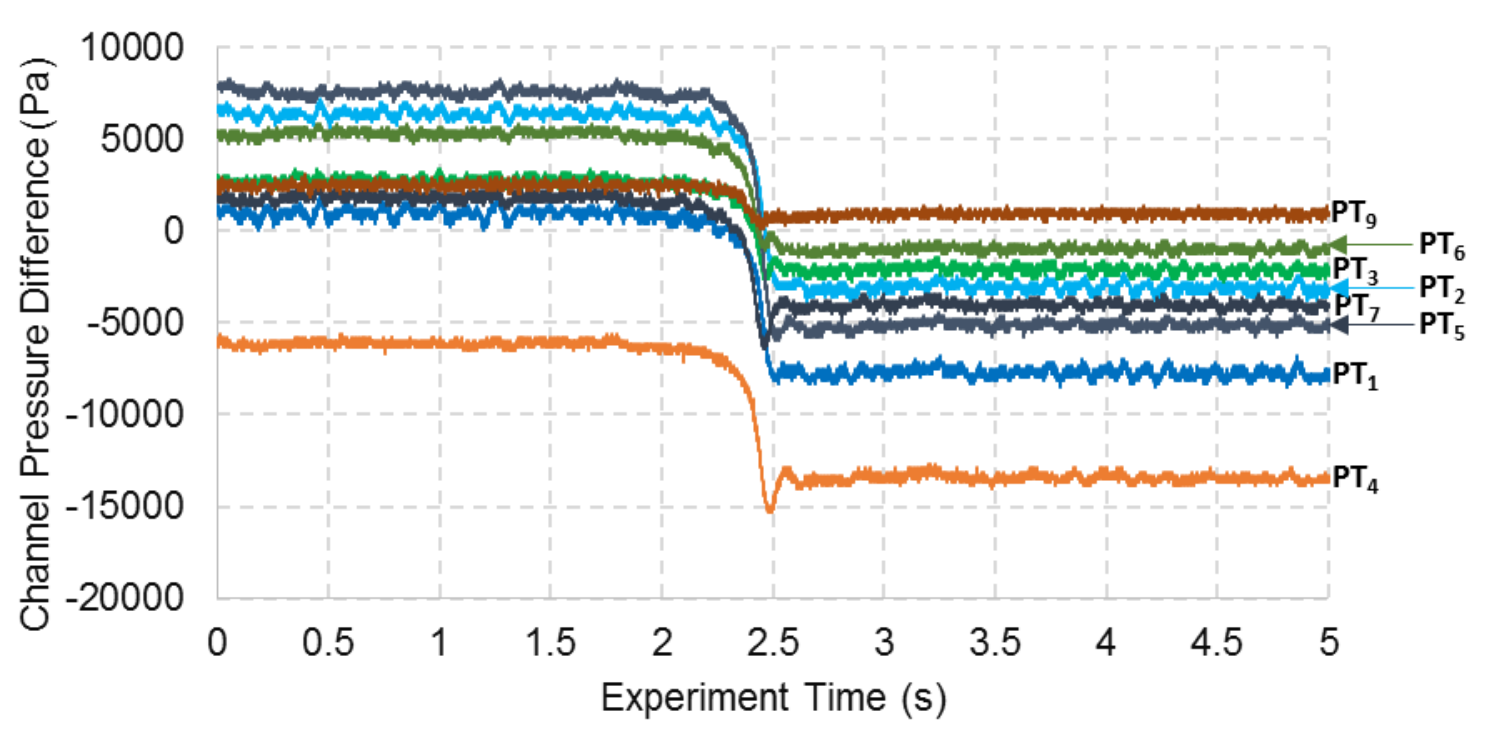

Figure 5.22. Pressure difference change during plate snap with lasers at axial location $\mathbf{E}$ and flow rate of $2.927 \mathrm{~kg} / \mathrm{s}$.

From Figure 5.21 and Figure 5.22, it is apparent that both lasers and all eight axial pressure transducers registered evidence of the plate snap at the same time. Figure 5.21 shows just before the 2.5 second mark that the plate, which had been deflecting into the larger channel, abruptly moved into the smaller channel. In Figure 5.22, this abrupt 
change in the plate deflection is accompanied by a large shift in the pressure distribution on the plate. Note that all pressure transducers recorded a sharp drop in channel pressure difference, with most indicating a change in sign.

The second data set showing the plate snap occurred with the lasers located at axial location D (290.525 mm from the trailing edge) at a flow rate of $2.936 \mathrm{~kg} / \mathrm{s}$. This flow rate is nearly identical to the velocity seen in the snap at location E. Additionally, axial location $\mathrm{D}$ is closer to the half-way point on the plate than any other measurement location. In the following figures, a five second excerpt from the data with the snap included is presented.

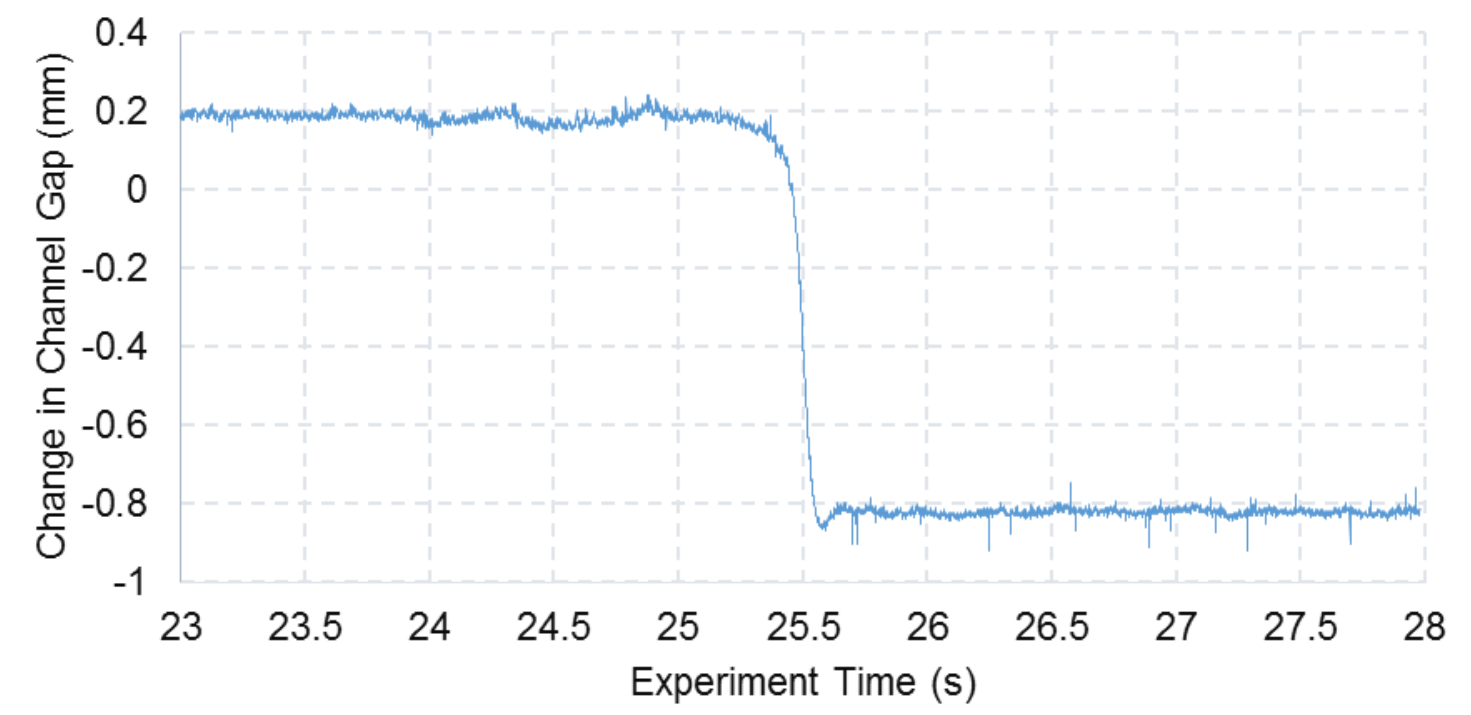

Figure 5.23. Change in smaller channel gap during plate snap at axial location $D$ and flow rate 2.936 $\mathrm{kg} / \mathrm{s}$ 


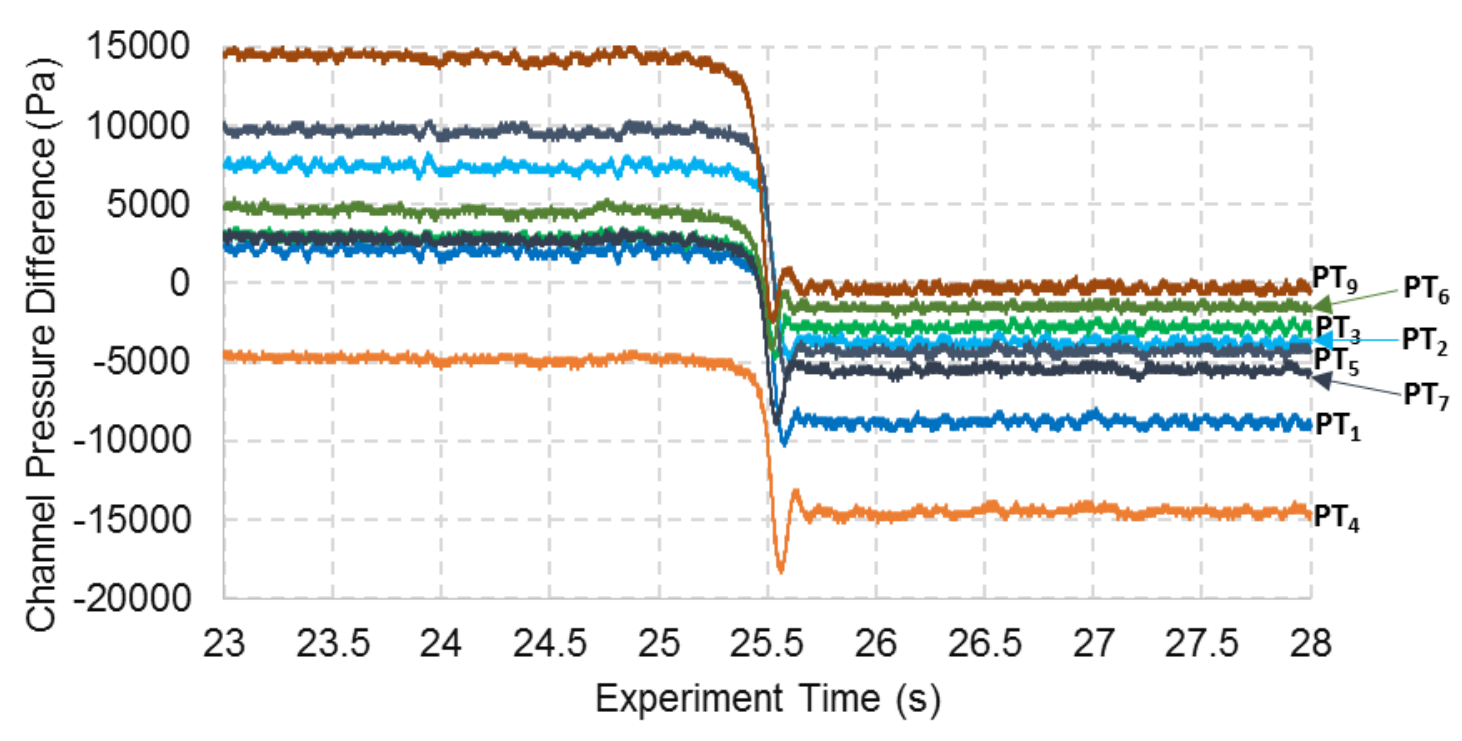

Figure 5.24. Pressure difference change during plate snap with lasers at axial location $D$ and flow velocity of $2.936 \mathrm{~kg} / \mathrm{s}$

As before, Figure 5.23 and Figure 5.24 show the response of the pressure transducers and the laser channel measurement when the plate snaps. Note that in Figure 5.23, data is available only for the laser monitoring the smaller channel as there was a loss of signal from the laser monitoring the larger channel. In both figures, the pressure transducers and the laser capture the snap of the plate around the 25.5 second mark. When considering the pressure difference data, it is clear that a large shift in the pressure difference at all axial locations occurred when the plate snapped.

Finally, if all the pressure data of Figure 5.13 through Figure 5.20 is re-plotted vs. axial location, with each series representing a 'pressure profile', it is possible to discern a clear group of pre-snap profiles and post-snap profiles, as shown in Figure 5.25. 


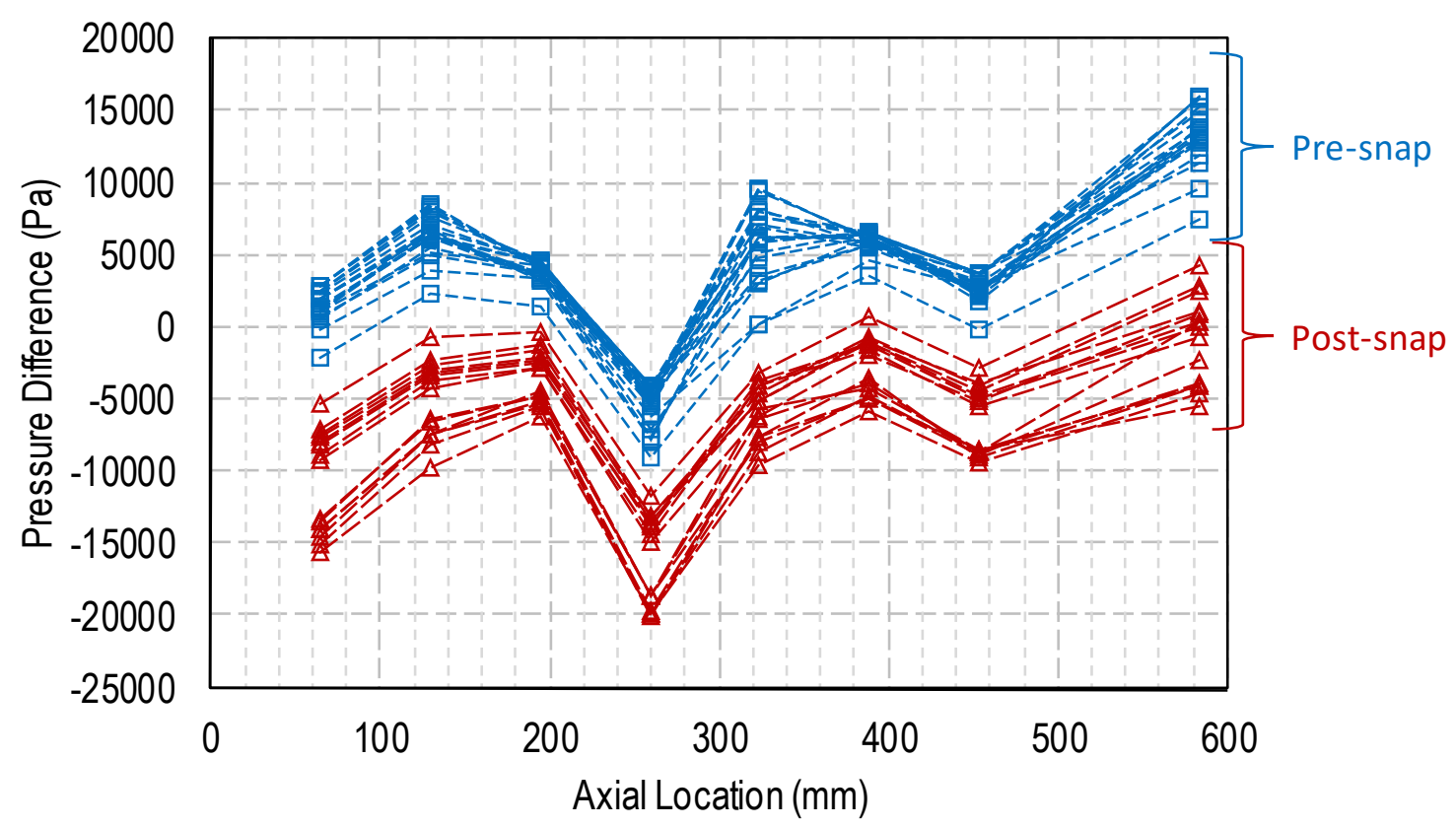

Figure 5.25. Pressure profiles. Blue lines are pre-snap data sets, orange lines are post-snap data sets. Positive pressure indicates higher pressure in the smaller channel

When examining all data sets, it appears that any time a plate snap is observed with the laser heads at any axial location all pressure sensors also register a large change in the measured pressure difference, at all axial locations. At the same time, there do not appear to be any changes in the pressure difference measurements when a plate snap is not directly observed with the laser heads. A qualitative explanation for why the pressure difference and channel gap can be inferred from the basic beam bending equation. From Eq. 2.5, it is clear that if the distributed load, caused by the pressure difference across the plate, (represented by P) were to change drastically, there would be a corresponding change in the beam deflection. In fact, if the sign of the distributed load were to change, the direction of the plate flexure would be expected to flip. 
Given the complex three-dimensional and tightly coupled nature of the flow and deflection in these experiments, Eq. 2.5 likely needs some modification to properly represent the flat plate geometry under consideration. However this expression provides a first order estimate of the expected behavior.

\section{4: Pinned-Edge Experiments}

\subsection{1: Flow Rates and Velocities}

Using the methodology described in section 4.4, the measured flow rates and corresponding calculated average channel velocities for the Pinned edge experiments are

provided in Table 5.5. Note that the average channel velocity is based on the assumption that the plexiglass walls are rigid and do not bow outward as the flow rate and pressure increases. 
Table 5.5. Measured flow rates and calculated average velocities for pinned edge experiments

\begin{tabular}{|c|c|c|c|c|c|c|c|c|c|c|c|c|c|}
\hline \multicolumn{14}{|c|}{ Laser Position } \\
\hline \multicolumn{2}{|c|}{$\begin{array}{l}\text { A - 0.500" } \\
\mathrm{kg} / \mathrm{s} \quad \mathrm{m} / \mathrm{s}\end{array}$} & \multicolumn{2}{|c|}{$\begin{array}{l}\text { B - 6.000" } \\
\mathrm{kg} / \mathrm{s} \quad \mathrm{m} / \mathrm{s}\end{array}$} & \multicolumn{2}{|c|}{$\begin{array}{c}\mathrm{C}-8.925^{\prime \prime} \\
\mathrm{kg} / \mathrm{s} \quad \mathrm{m} / \mathrm{s}\end{array}$} & \multicolumn{2}{|c|}{$\begin{array}{l}\text { D - 11.438" } \\
\mathrm{kg} / \mathrm{s} \quad \mathrm{m} / \mathrm{s}\end{array}$} & \multicolumn{2}{|c|}{$\begin{array}{l}\text { E - 18.675" } \\
\mathrm{kg} / \mathrm{s} \quad \mathrm{m} / \mathrm{s}\end{array}$} & \multicolumn{2}{|c|}{$\begin{array}{l}\text { F - 21.675" } \\
\mathrm{kg} / \mathrm{s} \quad \mathrm{m} / \mathrm{s}\end{array}$} & \multicolumn{2}{|c|}{$\begin{array}{l}\text { G - 25.000" } \\
\mathrm{kg} / \mathrm{s} \quad \mathrm{m} / \mathrm{s}\end{array}$} \\
\hline 1.030 & 2.040 & 1.046 & 2.071 & 1.034 & 2.048 & 1.041 & 2.063 & 0.997 & 1.975 & 0.980 & 1.941 & 1.043 & 2.067 \\
\hline 1.339 & 2.652 & 1.395 & 2.764 & 1.314 & 2.602 & 1.329 & 2.633 & 1.281 & 2.538 & 1.300 & 2.575 & 1.281 & 2.538 \\
\hline 1.662 & 3.293 & 1.745 & 3.455 & 1.648 & 3.264 & 1.699 & 3.364 & 1.648 & 3.264 & 1.657 & 3.283 & 1.648 & 3.264 \\
\hline 2.244 & 4.446 & 2.268 & 4.493 & 2.196 & 4.350 & 2.254 & 4.464 & 2.247 & 4.452 & 2.208 & 4.374 & 2.247 & 4.452 \\
\hline 2.570 & 5.090 & 2.502 & 4.957 & 2.462 & 4.877 & 2.537 & 5.026 & 2.578 & 5.106 & 2.454 & 4.861 & 2.578 & 5.106 \\
\hline 2.684 & 5.316 & 2.757 & 5.461 & 2.600 & 5.149 & 2.668 & 5.284 & 2.660 & 5.269 & 2.684 & 5.316 & 2.660 & 5.269 \\
\hline 2.759 & 5.464 & 2.884 & 5.713 & 2.779 & 5.505 & 2.728 & 5.404 & 2.859 & 5.663 & 2.768 & 5.483 & 2.859 & 5.663 \\
\hline 2.842 & 5.630 & 2.963 & 5.869 & 2.844 & 5.634 & 2.864 & 5.673 & 2.965 & 5.872 & 2.983 & 5.909 & 2.965 & 5.872 \\
\hline 3.312 & 6.561 & 3.419 & 6.772 & 3.221 & 6.380 & 3.241 & 6.419 & 3.312 & 6.560 & 3.305 & 6.546 & 3.312 & 6.560 \\
\hline 3.642 & 7.214 & 3.916 & 7.756 & 3.684 & 7.298 & 3.711 & 7.350 & 3.734 & 7.396 & 3.759 & 7.446 & 3.734 & 7.396 \\
\hline 4.154 & 8.228 & 4.254 & 8.426 & 4.129 & 8.178 & 4.096 & 8.114 & 4.086 & 8.093 & 4.161 & 8.242 & 4.086 & 8.093 \\
\hline
\end{tabular}

\subsection{2: Channel Gap Results}

Figure 5.26 through Figure 5.32 provide the measured channel gap and corresponding change in channel gap thickness at the seven axial laser positions (see Figure 4.3 and Table 4.3 for the measurement locations corresponding to each pair of plots). The change in channel gap is relative to an initial channel thickness, which was measured before each flow test. See Appendix B for the initial gap values. 

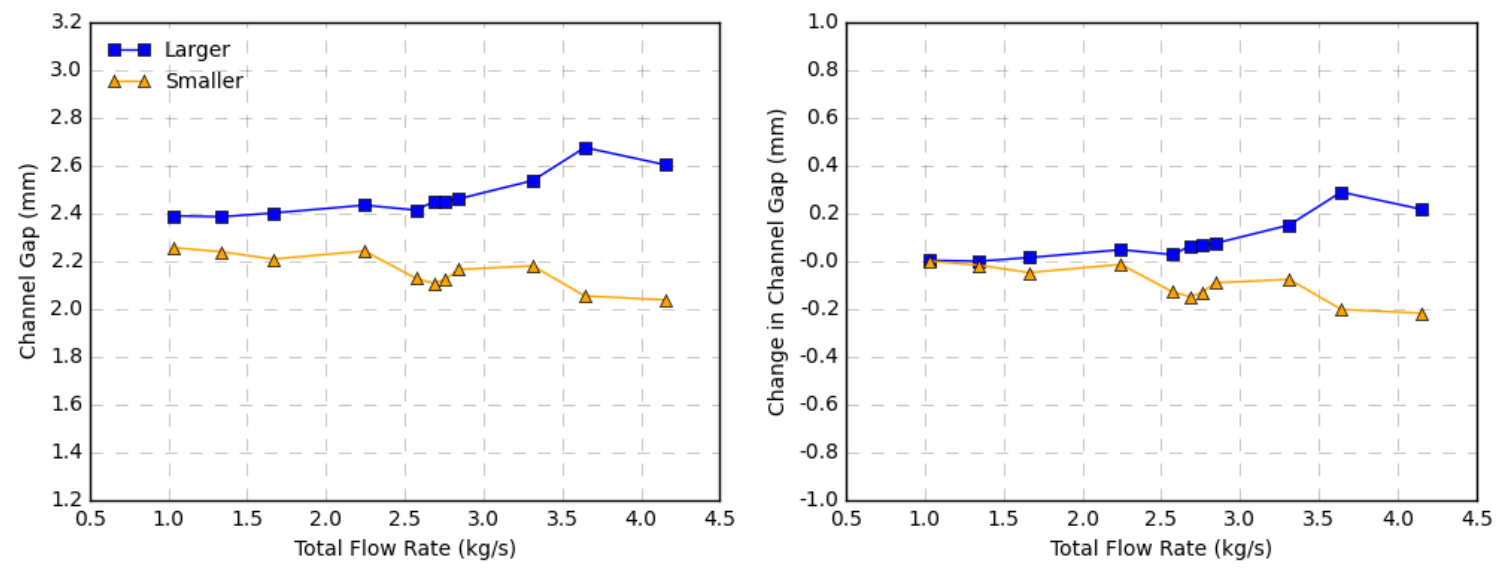

Figure 5.26. Channel gap and deflection at location $A-12.7 \mathrm{~mm}$ from trailing edge with pinned edges
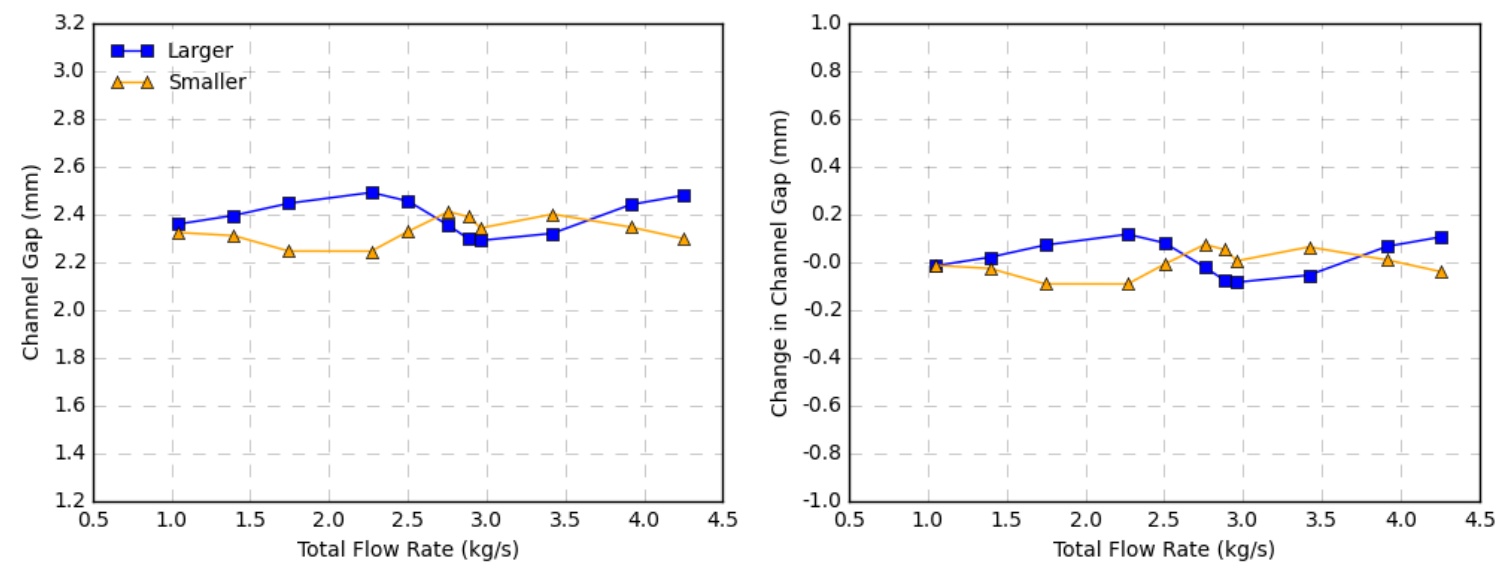

Figure 5.27. Channel gap and deflection at location $B-152.4 \mathrm{~mm}$ from trailing edge with pinned edges
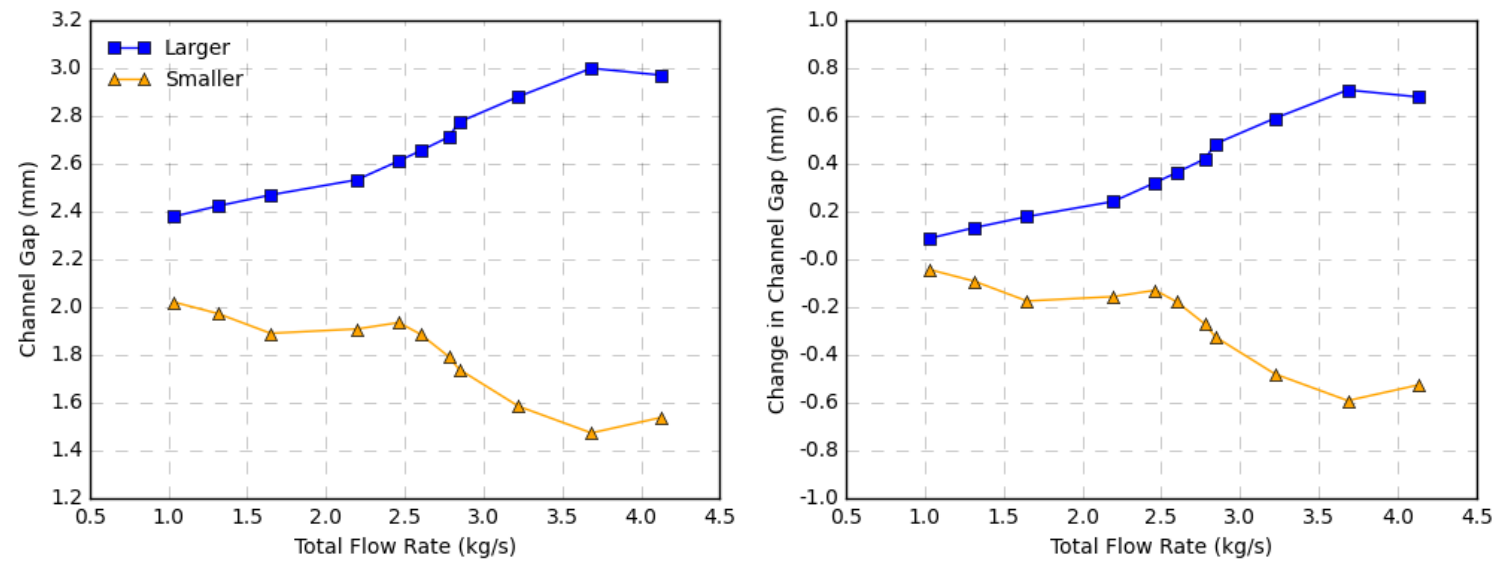

Figure 5.28. Channel gap and deflection at location $\mathrm{C}-226.7 \mathrm{~mm}$ from trailing edge with pinned edges 

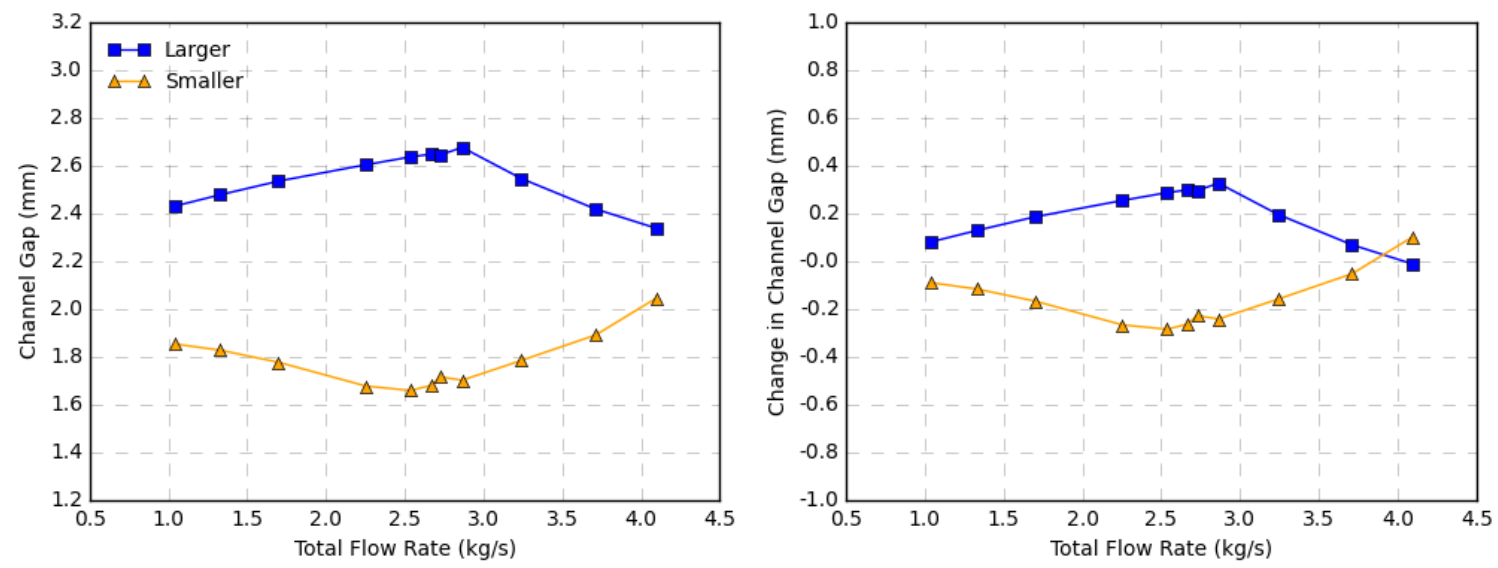

Figure 5.29. Channel gap and deflection at location $D-290.5 \mathrm{~mm}$ from trailing edge with pinned edges
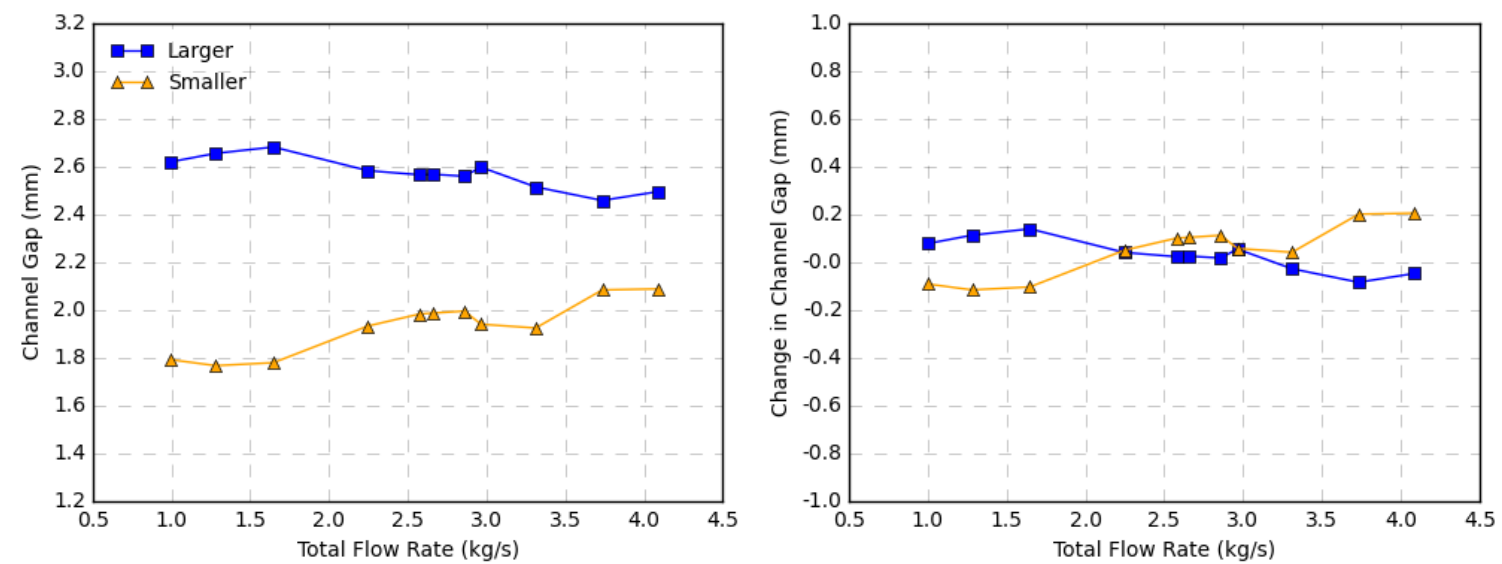

Figure 5.30. Channel gap and deflection at location $E-474.3 \mathrm{~mm}$ from trailing edge with pinned edges
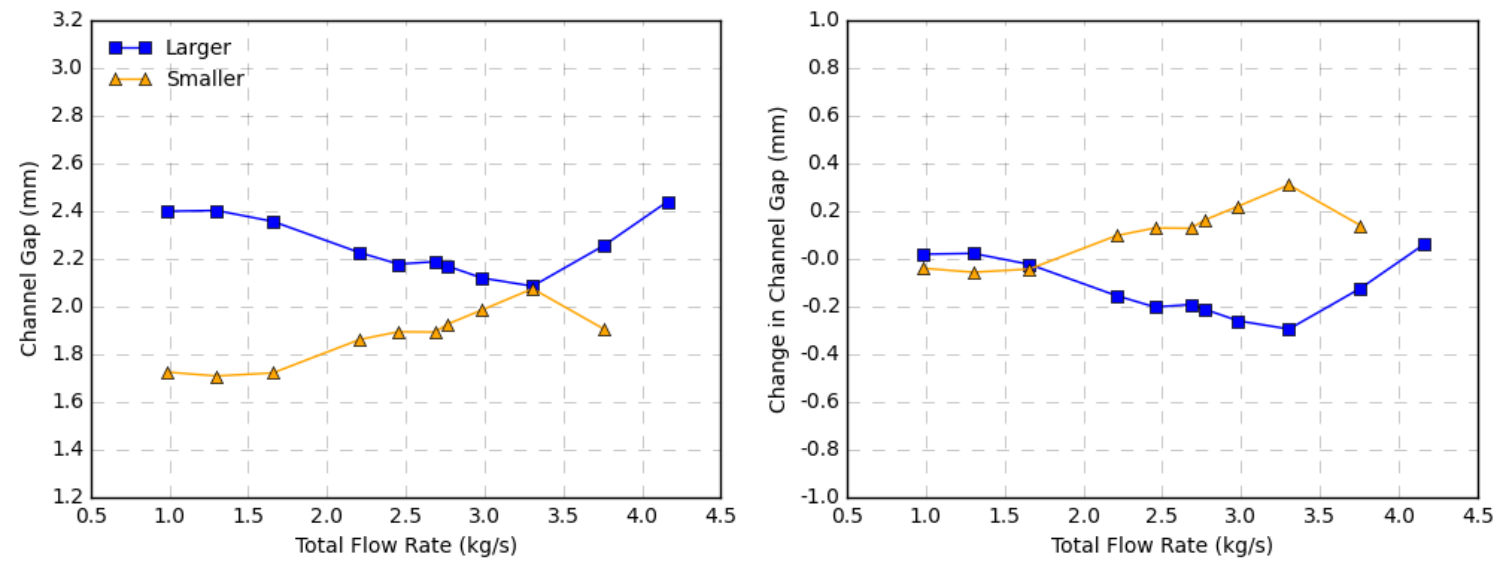

Figure 5.31. Channel gap and deflection at location $F-550.5 \mathrm{~mm}$ from trailing edge with pinned edges 

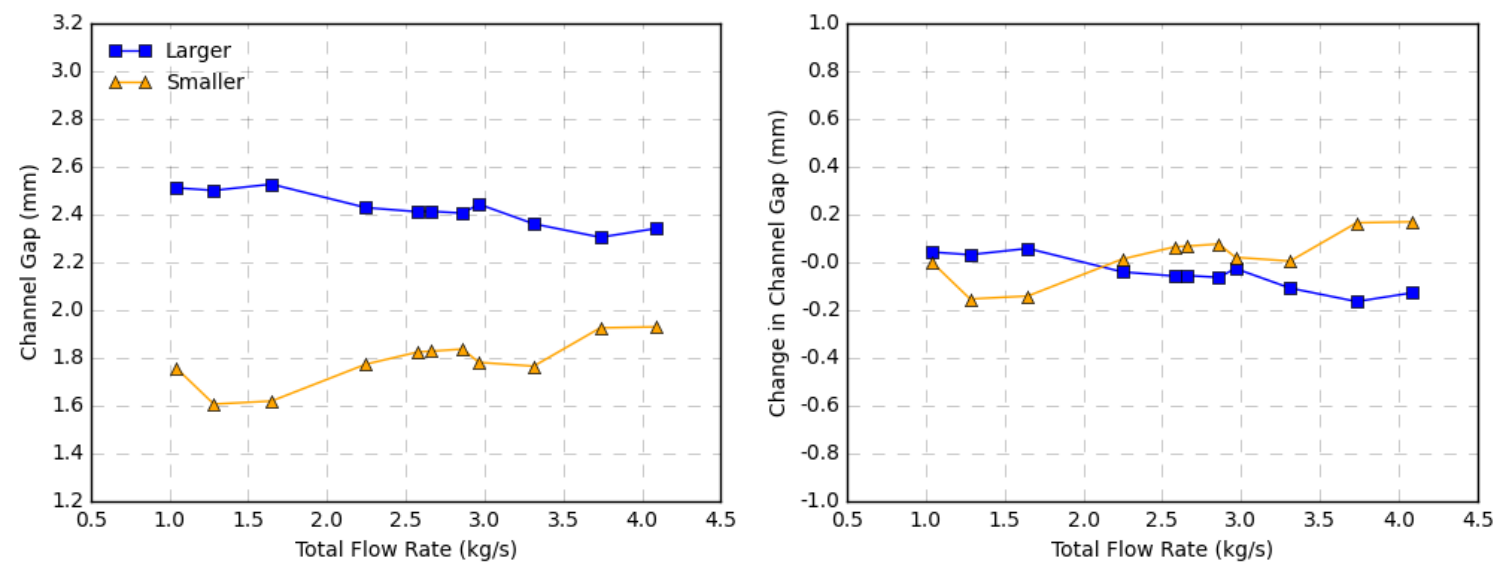

Figure 5.32. Channel gap and deflection at location $G-635.0 \mathrm{~mm}$ from trailing edge with pinned edges

Note that in Figure 5.31, no data is available from the laser monitoring the smaller channel at the highest flow rate. The laser sensor had difficulty maintaining an adequate signal during that single flow test. Using data for the change in the smaller channel thickness from the preceding figures, an estimation of the plate profile at various velocities is shown in Figure 5.33. Note that since the actual flow rates (see Table 5.5) varied somewhat among the different laser positions, an average of the calculated flow rates was used for the legend in Figure 5.33. Unlike the Free (Un-Pinned) edge experiments, these experiments showed no clear indication of a plate snap. Additionally, the magnitude of deflection was significantly lower in the presence of a comb. 


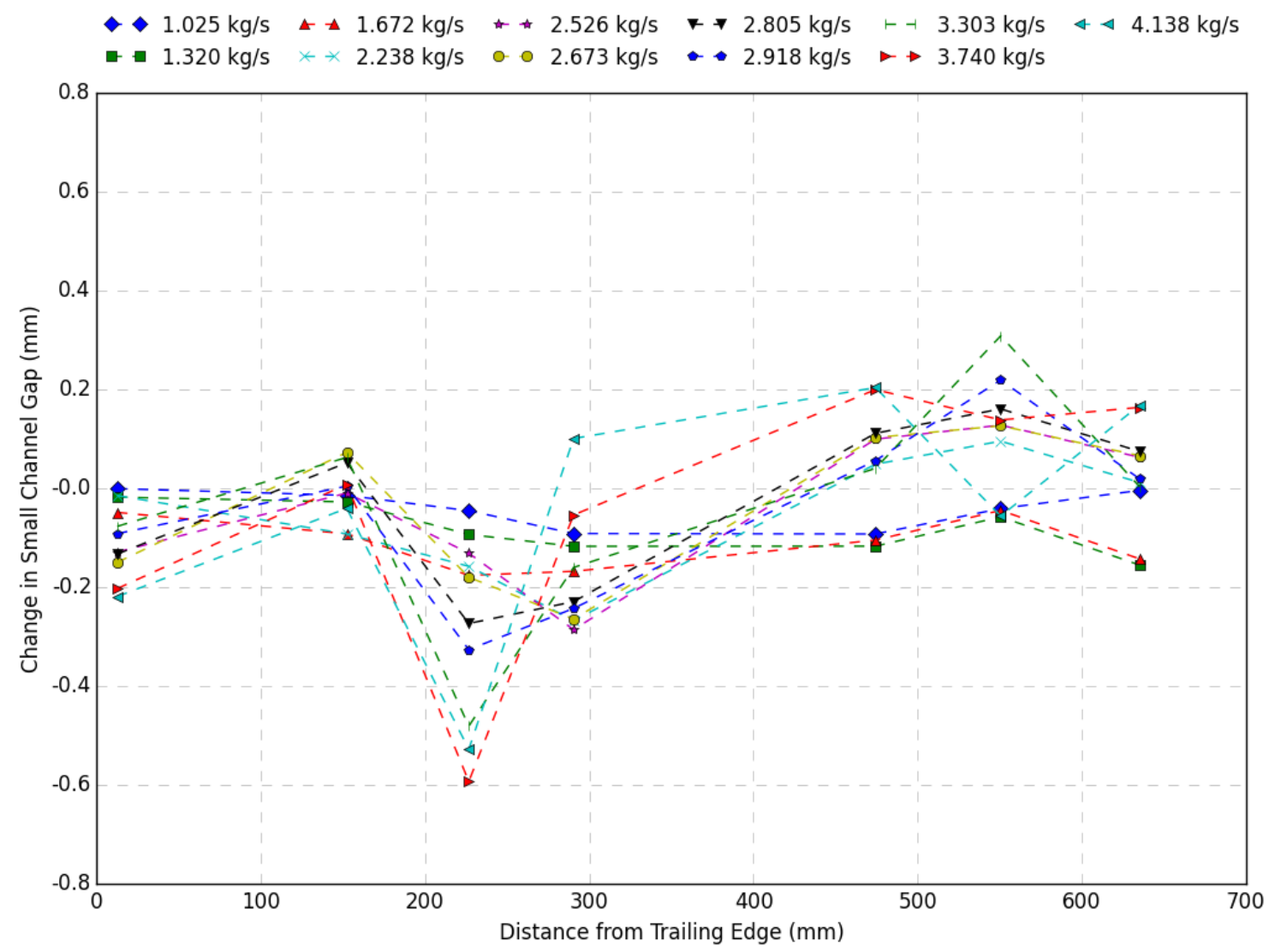

Figure 5.33. Plate profiles at various flow rates with pinned edges.

There appears to be a general increase in the magnitude of the deflection as the velocity increases. Additionally, the upper portion of the plate (locations E, F, and G) tend to indicate a plate deflection into the larger channel, whereas the lower portion of the plate (locations A, B, C, and D) indicate deflection into the smaller channel.

\subsection{3: Pressure Results}

In order to acquire laser data at all seven axial locations, the experiment was essentially repeated seven times with the lasers in a different location each time. Since the pressure taps remain in the same location for each experiment, there are essentially seven repeated trials of the same pressure data. 
Figure 5.34 through Figure 5.41 each represent one of the eight working pressure transducers (recall that Pressure Transducer 8 was inoperative). Each figure contains data from all seven trials $(A-G)$ for the specified pressure transducer. The trials are identified in the legend based on the location the lasers happened to be at for that trial, although the laser position should have no bearing on pressure results.

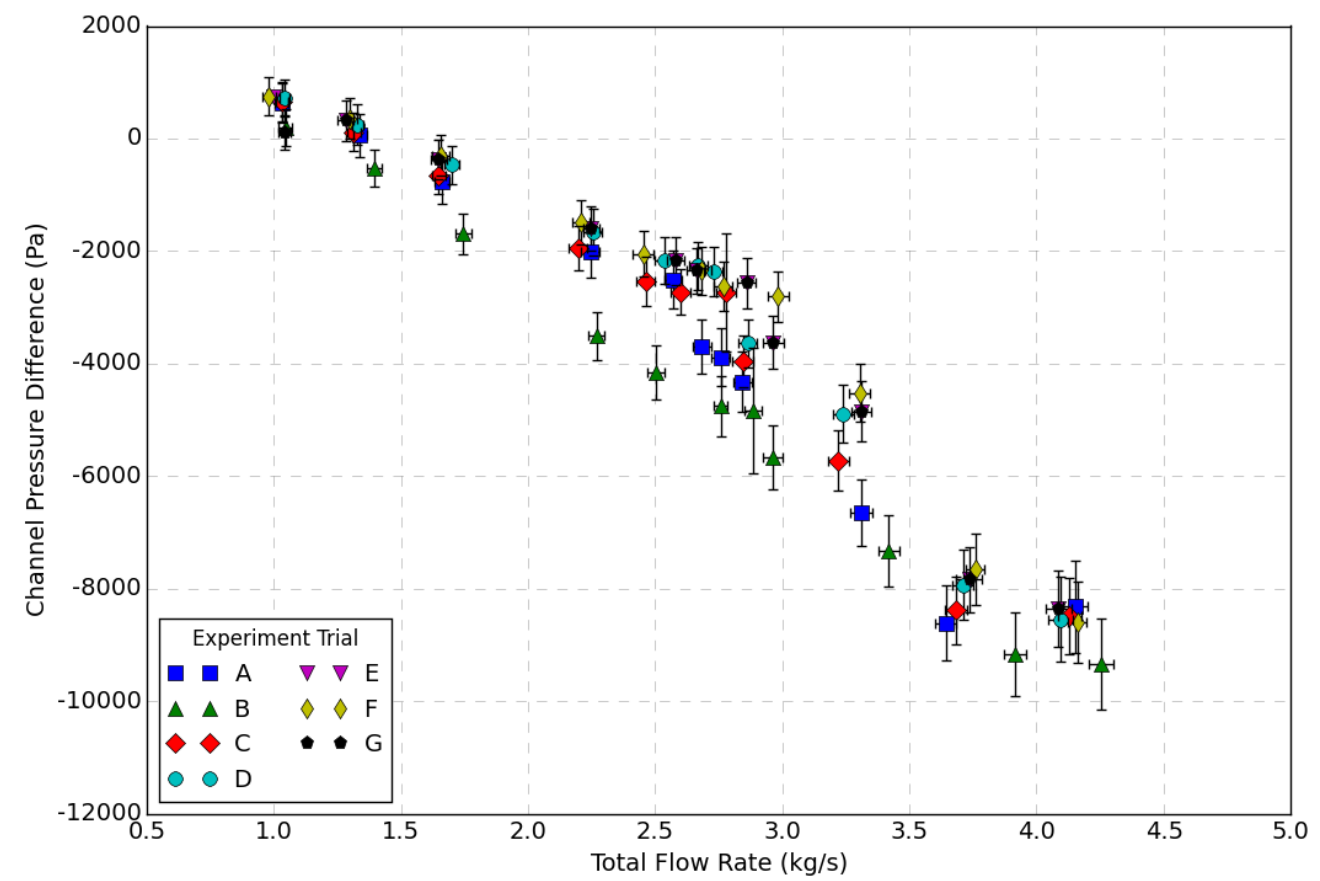

Figure 5.34. Channel pressure difference in pinned edge experiments, $64.77 \mathrm{~mm}(2.55$ ") from plate trailing edge (PT-1) 


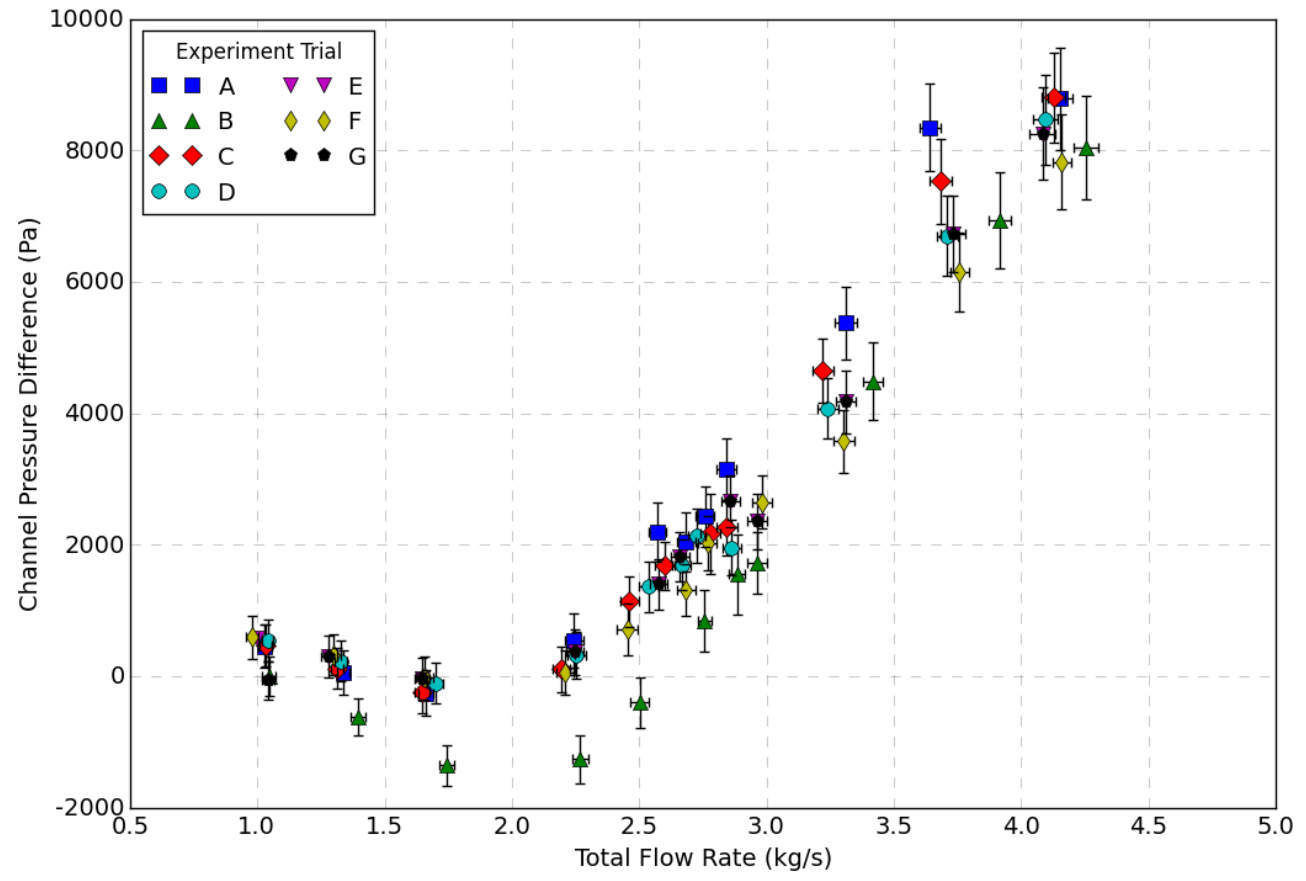

Figure 5.35. Channel pressure difference in pinned edge experiments, $129.54 \mathrm{~mm}\left(5.10^{\prime \prime}\right)$ from plate trailing edge (PT-2)

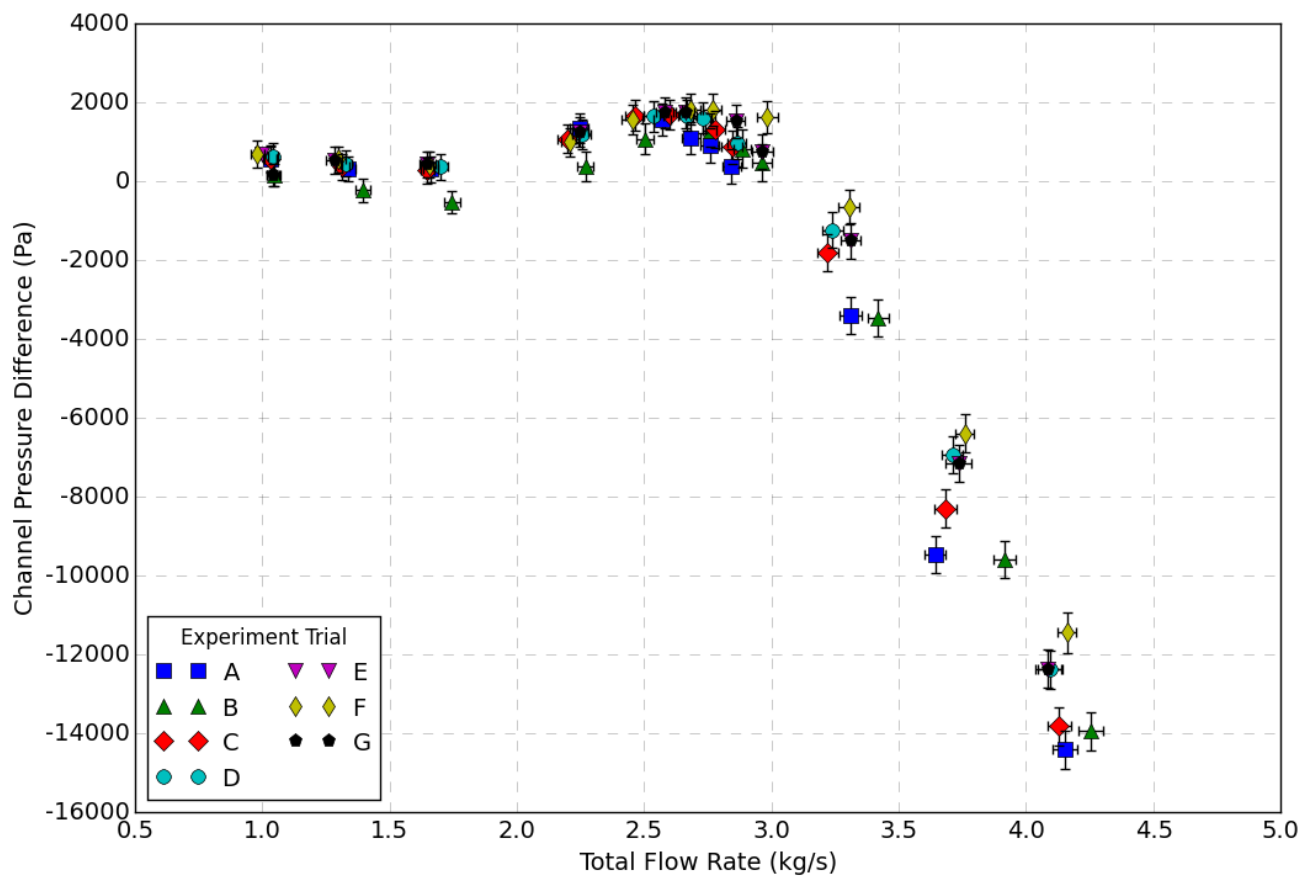

Figure 5.36. Channel pressure difference in pinned edge experiments, $194.31 \mathrm{~mm}\left(7.65^{\prime \prime}\right)$ from plate trailing edge (PT-3) 


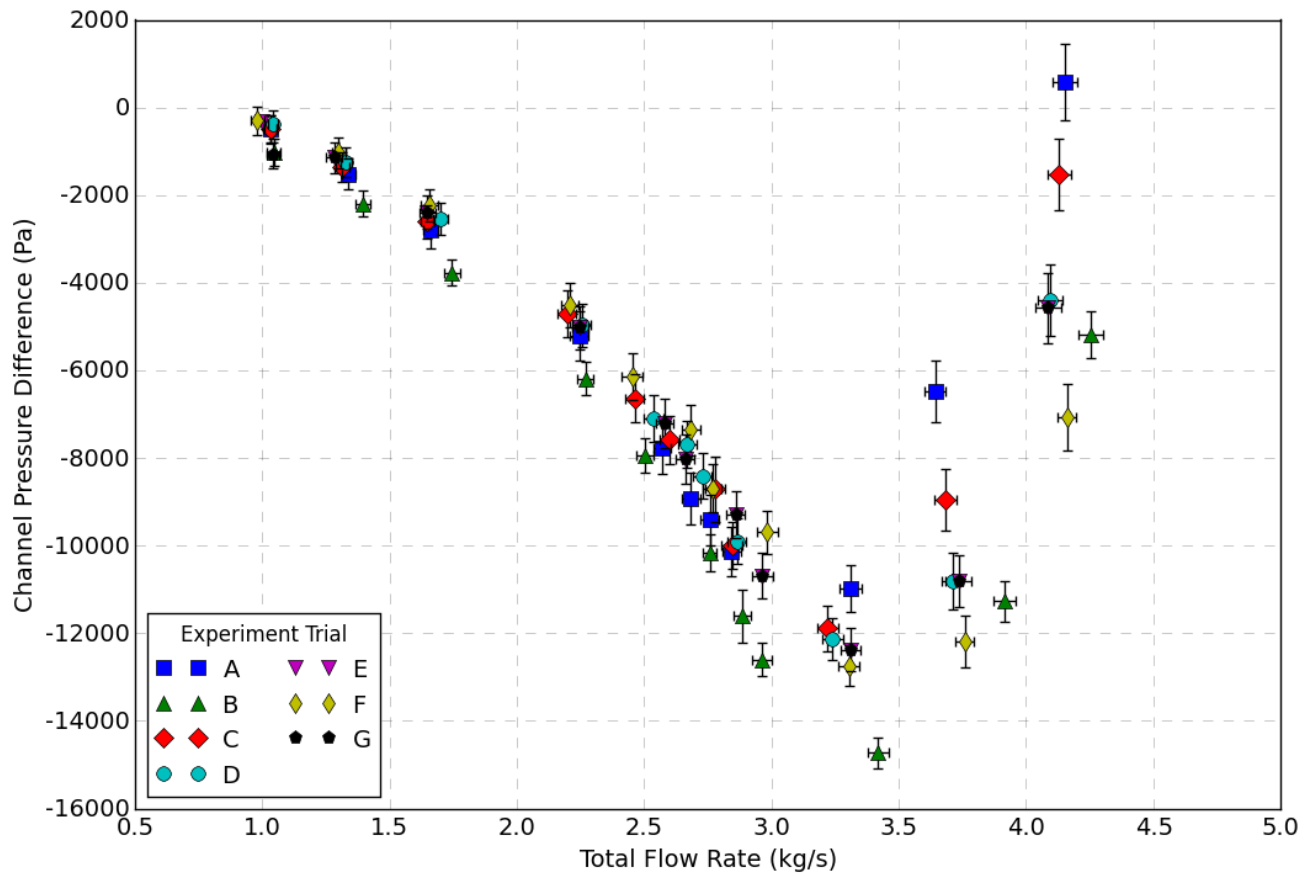

Figure 5.37. Channel pressure difference in pinned edge experiments, $259.08 \mathrm{~mm}(10.20 ")$ from plate trailing edge (PT-4)

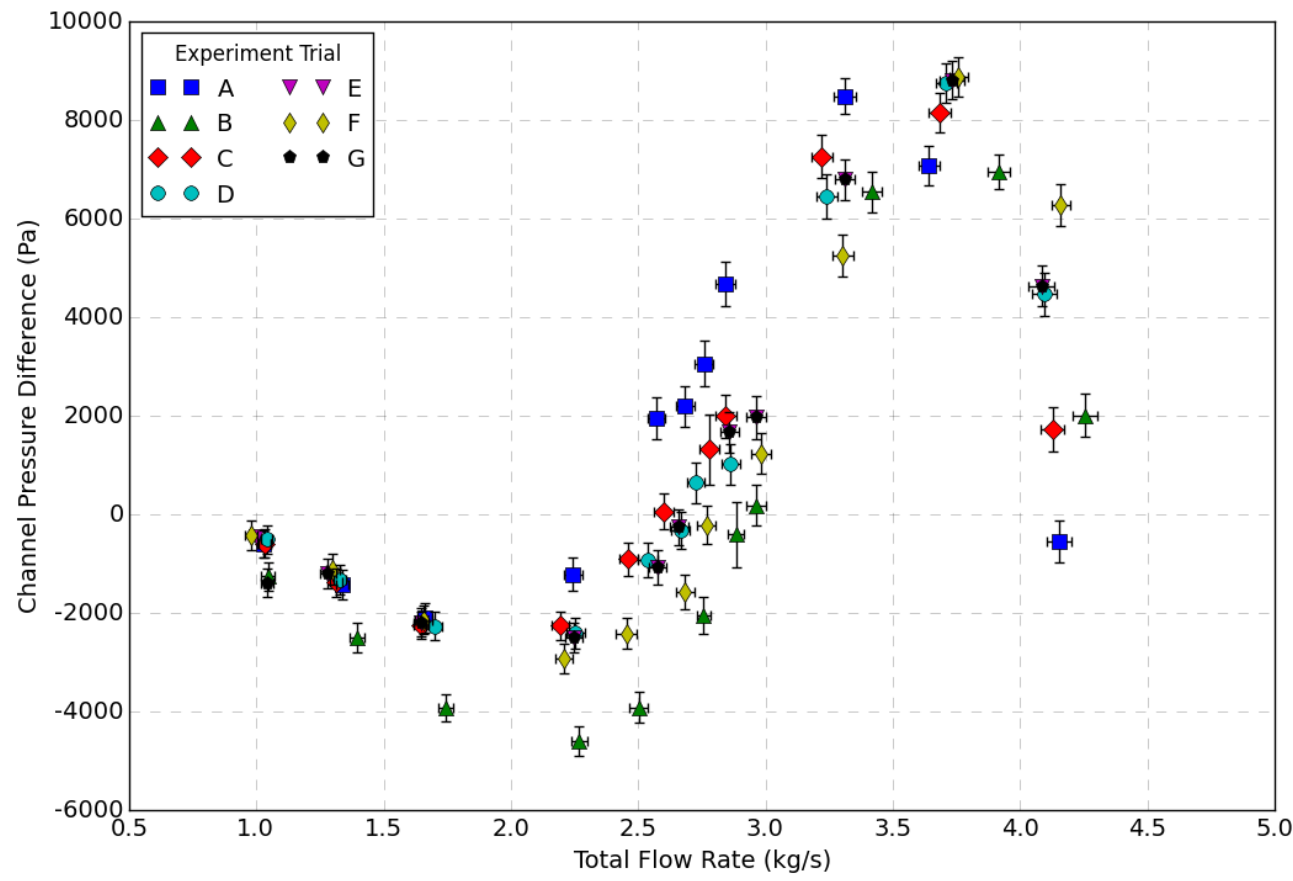

Figure 5.38. Channel pressure difference in pinned edge experiments, $323.85 \mathrm{~mm}(12.75 ")$ from plate trailing edge (PT-5) 


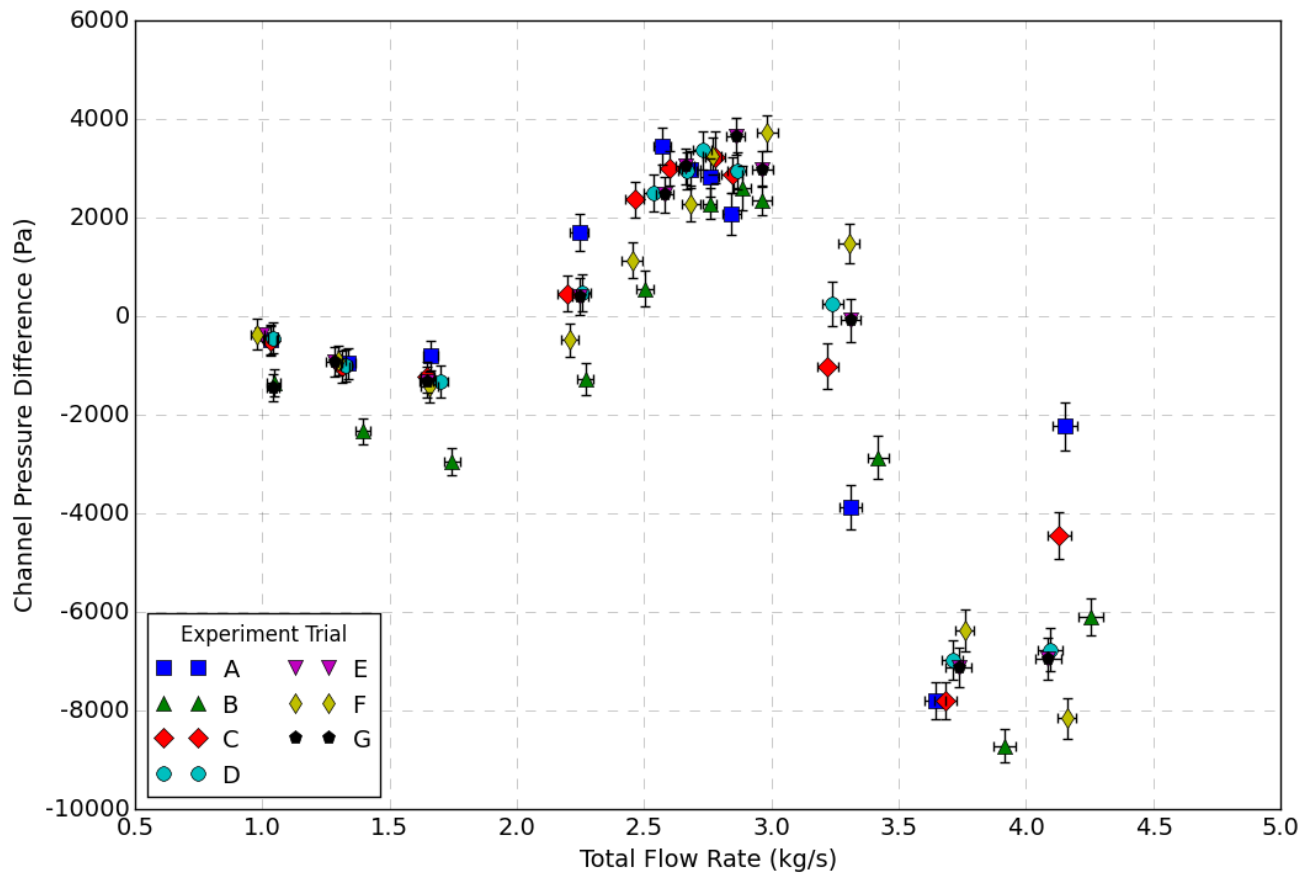

Figure 5.39. Channel pressure difference in pinned edge experiments, $388.62 \mathrm{~mm}(15.30 ")$ from plate trailing edge (PT-6)

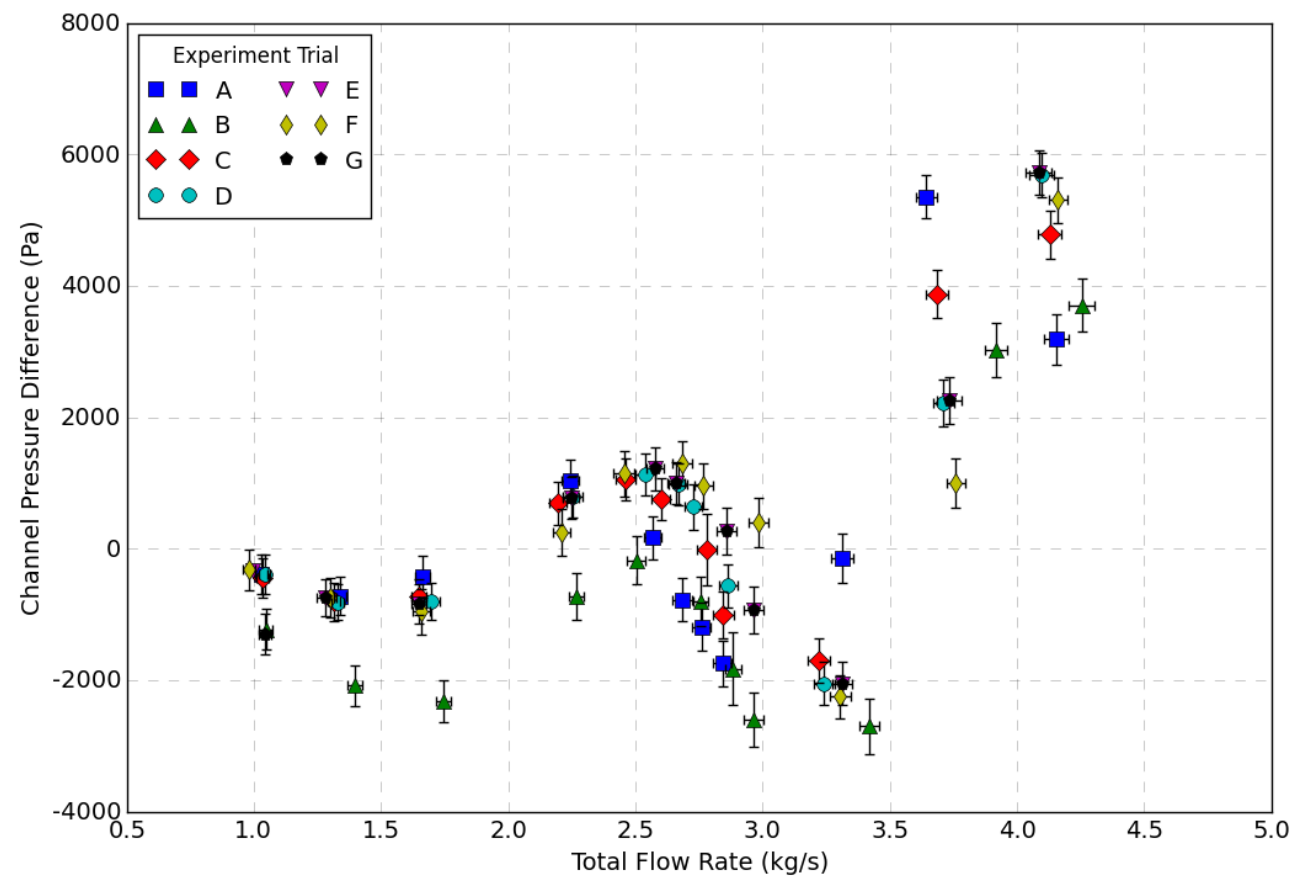

Figure 5.40. Channel pressure difference in pinned edge experiments, $453.39 \mathrm{~mm}(17.85 ")$ from plate trailing edge (PT-7) 


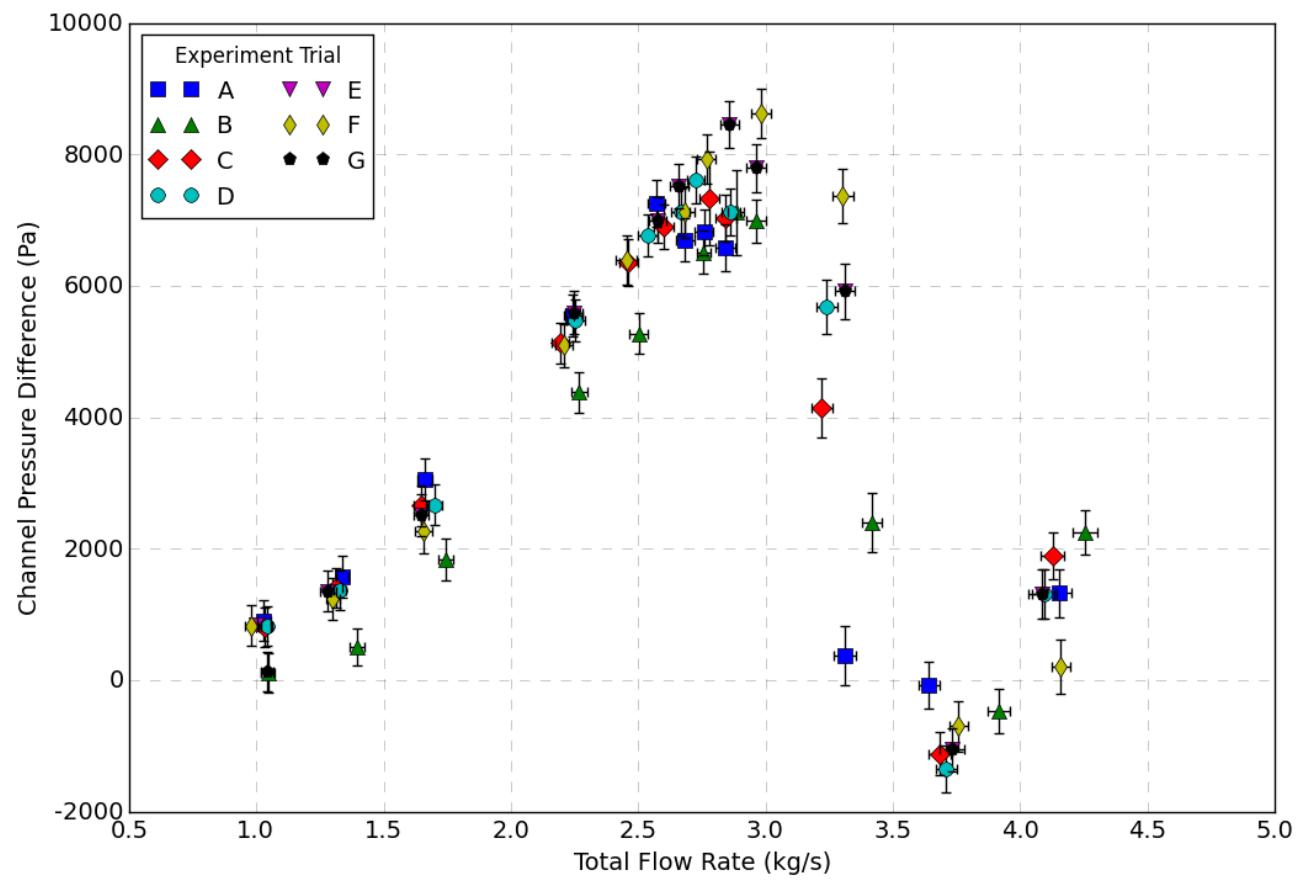

Figure 5.41. Channel pressure difference in pinned edge experiments, $582.93 \mathrm{~mm}(22.95 ")$ from plate trailing edge (PT-9)

With the pins in place, the pressure data between trials is highly repeatable. All of the preceding figures show close matching among trials. Also, with the pins in place, there is no indication in the pressure data of a plate snap, although the magnitude of the pressure does change significantly with flow velocity. Finally, each of the pressure transducers shows a unique trend in the pressure data, indicating that as the plate deforms - even a few mils - there is potential for a significant yet repeatable change in the resulting pressure field. 


\section{CHAPTER 6: EXPERIMENT CONCLUSIONS}

\section{1: Tolerances}

When designing the experiment, a plate offset of $0.254 \mathrm{~mm}$ (10 mils) was targeted, giving ideal channel thicknesses of $2.54 \mathrm{~mm}$ (100 mils) and $2.032 \mathrm{~mm}$ (80 mils). From Table 5.1, the average thickness of the channels was measured to be approximately 2.44 mm (96 mils) and $2.14 \mathrm{~mm}$ (84 mils). Therefore, when considering the average channel thickness, the measured values are less than $0.127 \mathrm{~mm}(5 \mathrm{mils})$ from the targeted values. However, when considering the mapping of the channels shown in Figure 5.2, the channels vary about this average by approximately $0.381 \mathrm{~mm}( \pm 15 \mathrm{mils})$. These variations within the channels are so large in one area that the ideally smaller channel is actually larger.

When considering the sources of channel thickness variation, there are two main components: variation in the surface of the plexi-glass panels and relative flatness of the plate. With the flat test section, there is little that can be done about the flatness of the plate. Given that a flat plate is inherently less rigid than a curved plate, it will be exceptionally difficult to correct channel variation resulting from the flat plate. With regard to the plexi-glass, variations in the surface may be corrected through refinishing the surface or replacing the plexi-glass altogether.

From Figure 5.3, the sum of the channel thicknesses gives at least a rough approximation of the bulk behavior of the two panels together. From this figure, if we take that both panels together provide a variation of the total channel thickness of $\sim 0.5 \mathrm{~mm}( \pm 20 \mathrm{mils})$, 
we can reasonably assume that each panel contributes approximately $\sim 0.25 \mathrm{~mm}( \pm 10$ mils) of variation to the channel thickness. Also from the figure, there is a noticeable decrease of the channel thickness when moving from the side edges to the axial centerline. This inward curvature has been noted visually during assembly and disassembly of the test section, and is believed to have developed gradually over time as a result of water absorption by the plexi-glass. The influence of this plexi-glass variation could be significantly reduced by replacing the plexi-glass panels. The new panels should be milled down under high tolerances to provide a smooth, flat surface. Additionally, alternative materials should be considered which are not as susceptible to water absorption.

For the MURR LEU FSD core design, the thickness tolerance for channels 2-24 is $\pm 0.2032 \mathrm{~mm}( \pm 8 \mathrm{mils})$ about a targeted value of $2.3368 \mathrm{~mm}$ (92 mils) [46]. When considering the average channel thicknesses of $2.44 \mathrm{~mm}$ (96 mils) and $2.14 \mathrm{~mm}$ (84 mils), the experiment initially appears to be within the stated tolerance of the MURR LEU assembly. However, when considering that variation within a channel is approximately $0.381 \mathrm{~mm}( \pm 15 \mathrm{mils})$, the tolerances within the experiment are seen to be well outside those of the MURR LEU specifications. If the plexi-glass were replaced with new, machined flat panels, the variation would likely fall back within the MURR tolerances.

Additionally, for the MURR plates themselves, several factors contribute to the plate thickness tolerances. From Figure 6.1, the tolerances related to the fuel meat, Zr, and clad are shown. Given that the plates used in these experiments are homogenous 
aluminum rather than a multilayer composite, the total thickness variation is quite small well within the tolerances for an actual fuel plate. While the variation of the plate used for these experiments has not yet been quantified, previous work with plates from the same batch showed variations in the thickness of under $0.0254 \mathrm{~mm}$ (1 mil).

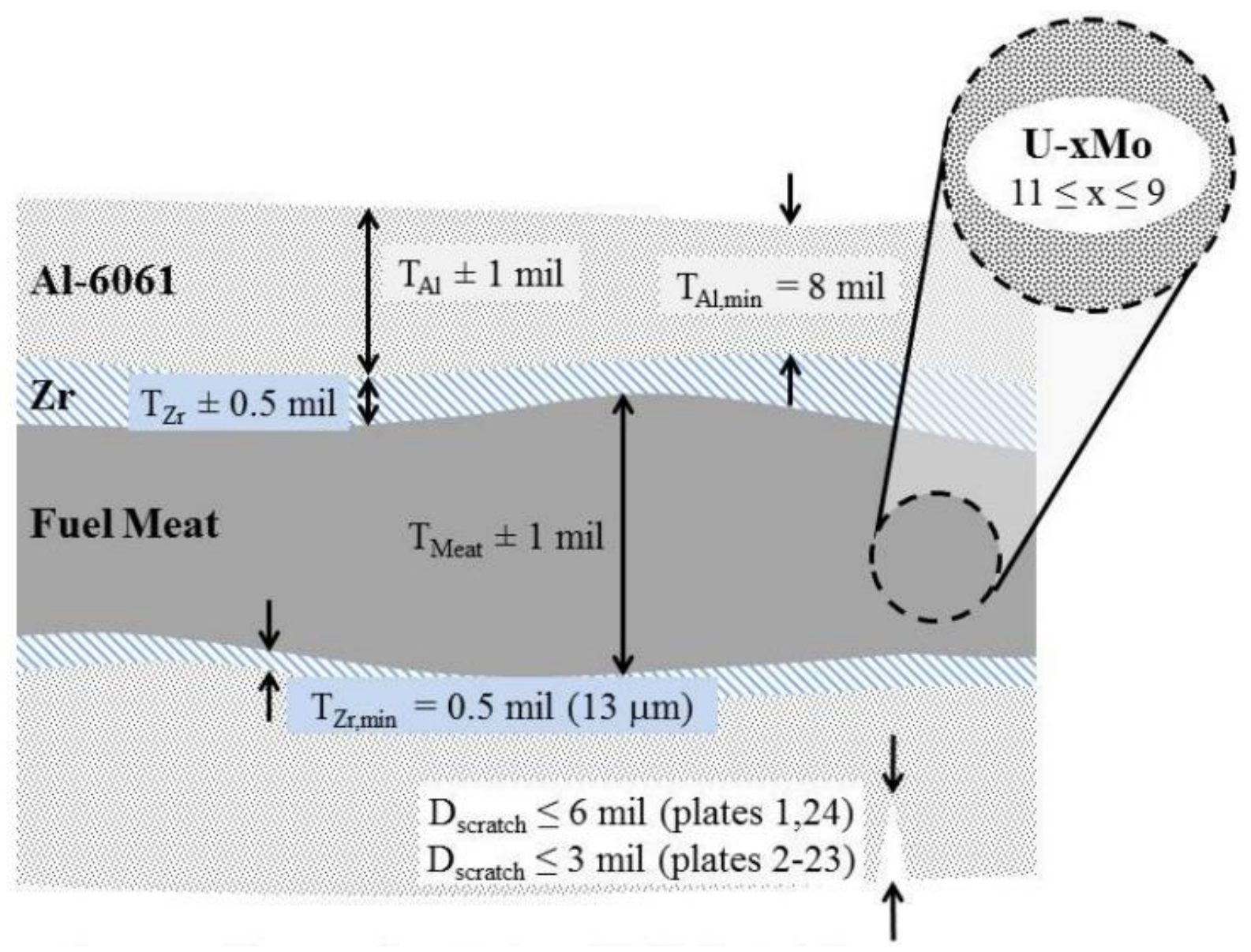

Figure 6.1. Cross sectional view of proposed MURR LEU plate. Provided as Figure 1 in reference [46] 


\section{2: Comparison to Literature}

\subsection{1: Zabriske}

While the results obtained by Zabriskie were largely qualitative, his experiments did have several similarities to the experiments presented here. In studying a $1.016 \mathrm{~mm}$ plate, he noted that the plate assumed a bulk deflection into one channel or the other, and did not simultaneously deflect into both directions. Also in those tests, he observed a sudden snap of the plate from deflection in one direction to the other direction, much like was observed here. What is more interesting is that he observed this while trying to use equally sized channels, whereas the channels in these experiments were intentionally biased. Finally, in looking at the variations in the channel thicknesses of these experiments, it has been hypothesized that the outer plexi glass panels could have absorbed some water, causing an inward bowing of the plexi-glass. This is largely consistent with Zabriskies observations after submerging a plexi-glass plate in water and tracking its deformation and increase in thickness over time [21].

\subsection{2: Miller}

In section 2.1.1 it was noted that the historical precedent for estimating a critical flow velocity comes from Miller [11]. One of the disadvantages of Miller's model is that it depends on both fluid channels on either side of a plate being the same thickness. While the results presented here focus on a plate offset by approximately $0.254 \mathrm{~mm}(10 \mathrm{mils})$, it is useful to evaluate these results in the context of the Miller Critical Velocity. 
A range of channel thickness values (parameter $h$ in Equation 2.2) relating to these experiments have been selected and Miller's Critical Velocity has been calculated. The critical velocity has been calculated using both the ideal and measured channel thickness values of Table 5.1. This provides six channel thickness values about which an estimate of the critical velocity can be calculated. Table 6.1 shows the estimates of $\mathrm{V}_{\mathrm{C}}$ for a plate with free (un-pinned) leading and trailing edges using these various channel gap values.

Table 6.1. Miller critical velocity calculation for various channel thicknesses

\begin{tabular}{ccccc}
\hline Case & $\mathbf{h}(\mathbf{m m})$ & $\mathbf{h}(\mathbf{m i l s})$ & $\mathbf{V}_{\mathbf{C}}(\mathbf{m} / \mathbf{s})$ & $\dot{\mathbf{m}}_{\mathbf{C}}(\mathbf{k g} / \mathbf{s})$ \\
\hline Ideal Geometry - Small Channel & 2.032 & 80 & 5.788 & 2.588 \\
Ideal Geometry - Average Channel & 2.286 & 90 & 6.139 & 3.088 \\
Ideal Geometry - Large Channel & 2.540 & 100 & 6.471 & 3.616 \\
Mapped Geometry - Small Channel & 2.143 & 84.4 & 5.944 & 2.803 \\
Mapped Geometry - Average Channel & 2.294 & 90.3 & 6.149 & 3.103 \\
Mapped Geometry - Large Channel & 2.444 & 96.2 & 6.347 & 3.413 \\
\hline
\end{tabular}

From this table, the critical total flow rate varies from approximately 2.6 to $3.6 \mathrm{~kg} / \mathrm{s}(5.7$ to $6.5 \mathrm{~m} / \mathrm{s}$ ), depending on the specified values for the gap thickness, h. From the free (un-pinned) edge experiments, Figure 5.11 shows the channel gap results for the point nearest the leading edge vs. the inlet flow rate. Using the average experiment channel thickness of $2.294 \mathrm{~mm}$ (90.3 mils), the resulting Miller Critical Velocity is $6.149 \mathrm{~m} / \mathrm{s}$. This equates to a total flow rate through both channels of $3.103 \mathrm{~kg} / \mathrm{s}$, with flow through a single channel of $1.551 \mathrm{~kg} / \mathrm{s}$. By dividing the measured flow rate by this 'Critical' flow rate through a single channel, Figure 5.11 can be re-plotted to give the channel gap measurements as a fraction of the Miller Critical Velocity. 

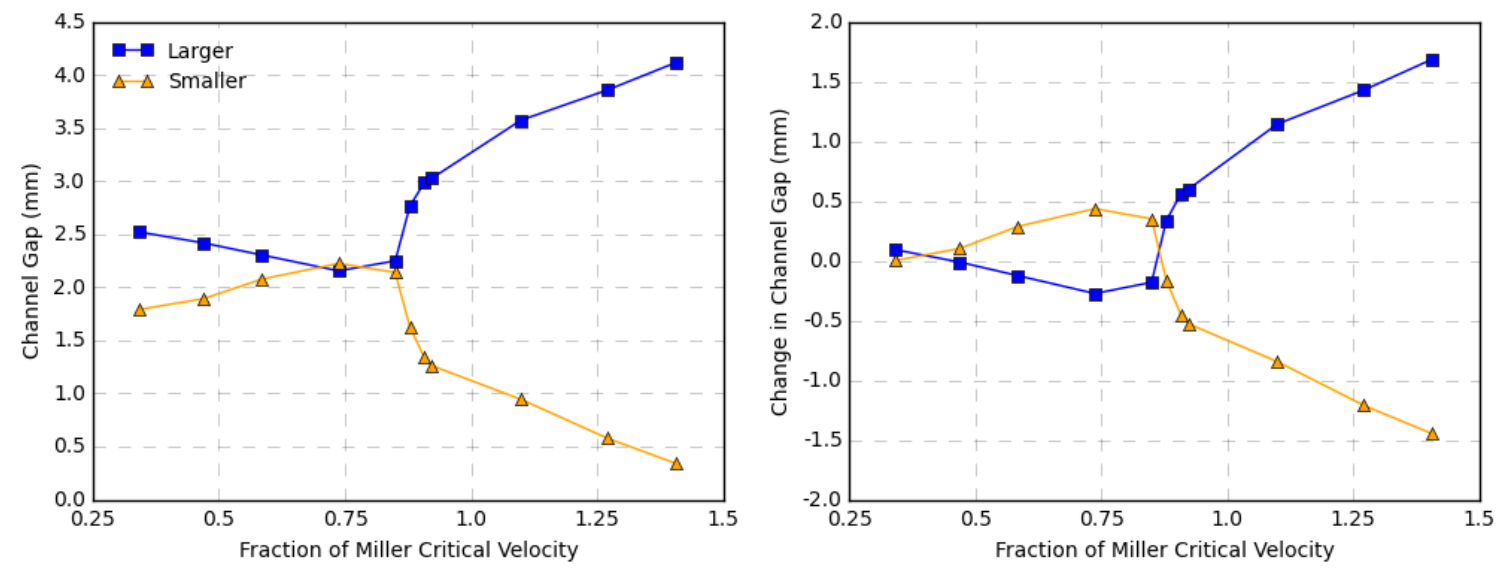

Figure 6.2. Channel gap vs. fraction of Miller Critical Velocity at $635 \mathrm{~mm}\left(25^{\prime \prime}\right)$ from the trailing edge with free leading and trailing edges

Figure 6.2 shows some deflection at points below Miller's critical velocity, further reinforcing that it does not apply to this scenario. This deflection is expected, as the plate offset should produce a stronger difference in pressure between the channels.

Additionally, the plate snap occurs below the Miller velocity, which is expected with an offset plate.

\subsection{3: Groninger}

Groninger observed significant plate deflection at velocities below the Miller critical velocity, and noted that the direction of deflection was not predictable. This was largely the result of equal sub-channels in his experiments [22]. The experiments discussed here differed from Groninger's in that the intentional plate offset resulted in a repeatable deflection pattern.

While Groninger speculated that small variations in the channel profiles significantly impacted the deflected shape of the plate, he had difficulty in quantifying these effects. Through the use of the laser measurement system, maps of the channels show significant 
variations. Therefore, the resulting plate profile and snapping behavior, while unusual, is not entirely surprising.

\section{3: Effect of Channel Variation on Expected Deflection}

Basic analytic models and previous numeric models have consistently shown the plate moving into the larger channel. This results from a larger pressure drop in the smaller channel due to its smaller hydraulic diameter. These earlier models however, did not consider the effect of variable channel thicknesses; rather, they assumed uniform thicknesses for the channels [44].

As noted in the channel mapping results, the channels are not of uniform thickness. In fact, there is a location approximately $225 \mathrm{~mm}$ (9 inches) from the trailing edge of the plate where the ideally 'smaller' channel is, in reality, the bigger channel. This area would cause a gradual flow contraction in one channel and a corresponding gradual flow expansion in the other channel. The effect that this would have on the flow distribution may be significant, and could have a large impact on the pressure distribution. 


\section{CHAPTER 7: CLUSTER DEVELOPMENT}

\section{1: Hardware Specifications}

Given the complexities of modeling tightly coupled systems using both computational fluid dynamics (CFD) and finite element analysis (FEA) models, it was first necessary to build the mini high performance compute cluster (HPCC) in Figure 7.1. The cluster consists of a server with over $5 \mathrm{~TB}$ of storage and 5 compute nodes. Combined, the compute nodes have 10 processors with a total of 52 processor cores and $384 \mathrm{~GB}$ of RAM. This provides a theoretical max of 659 gigaFlops. A map of the lab and cluster network is provided in Figure 7.2.

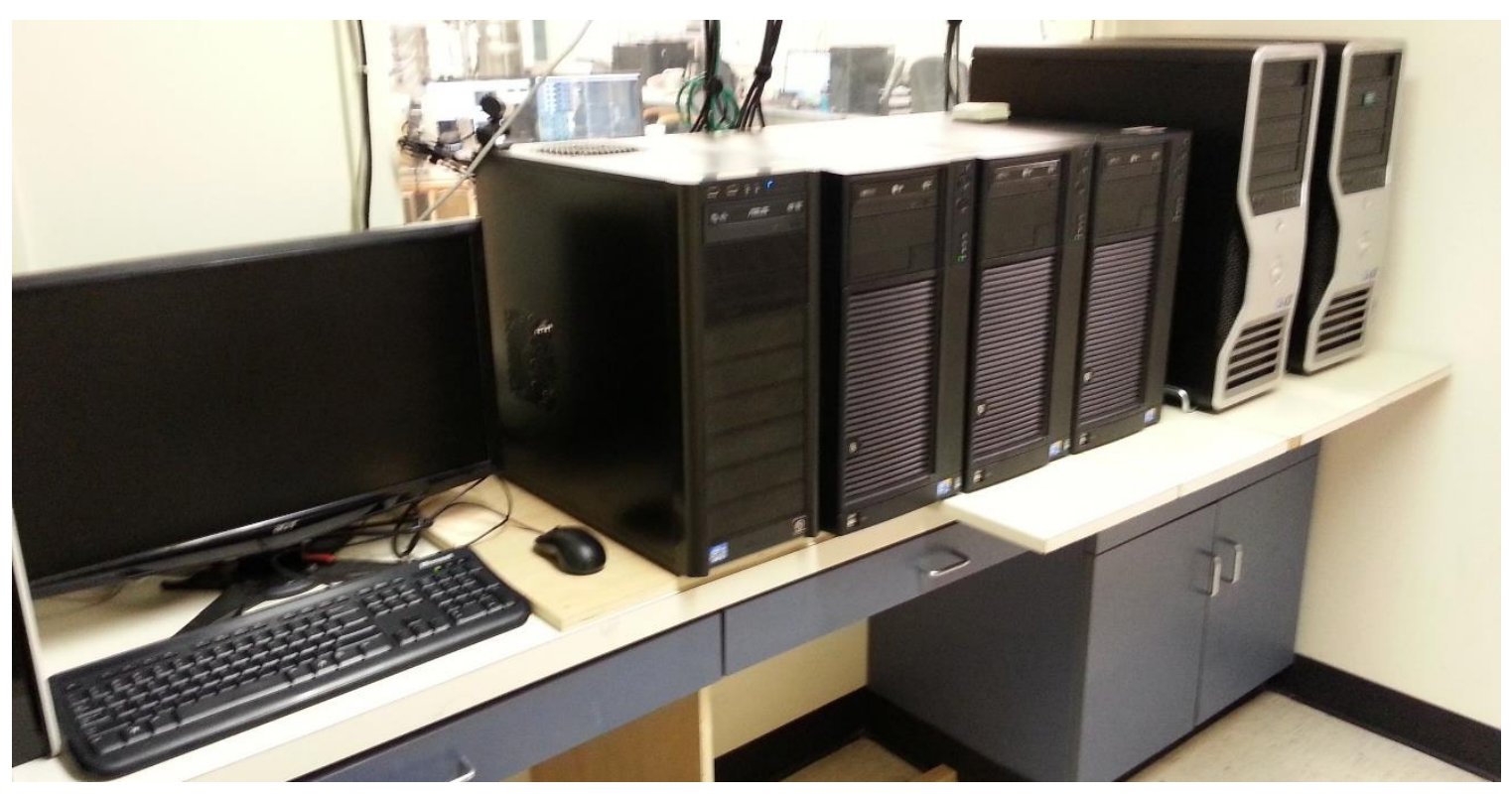

Figure 7.1. Mini high performance compute cluster (HPCC).

The cluster nodes run on Windows Server 2012 R2 with the HPC Pack. The cluster is designed for easy expansion with additional compute nodes, and is even capable of utilizing Windows 7/8 PCs for computations when they are not in use. Two of the nodes 
(the DELL workstations) are used primarily for pre- and post-processing, scratch storage, hosting the job scheduler, and hosting virtual machines for other projects. The primary codes available on the cluster are Star-CCM+ for CFD simulations and Abaqus for FEA simulations. 


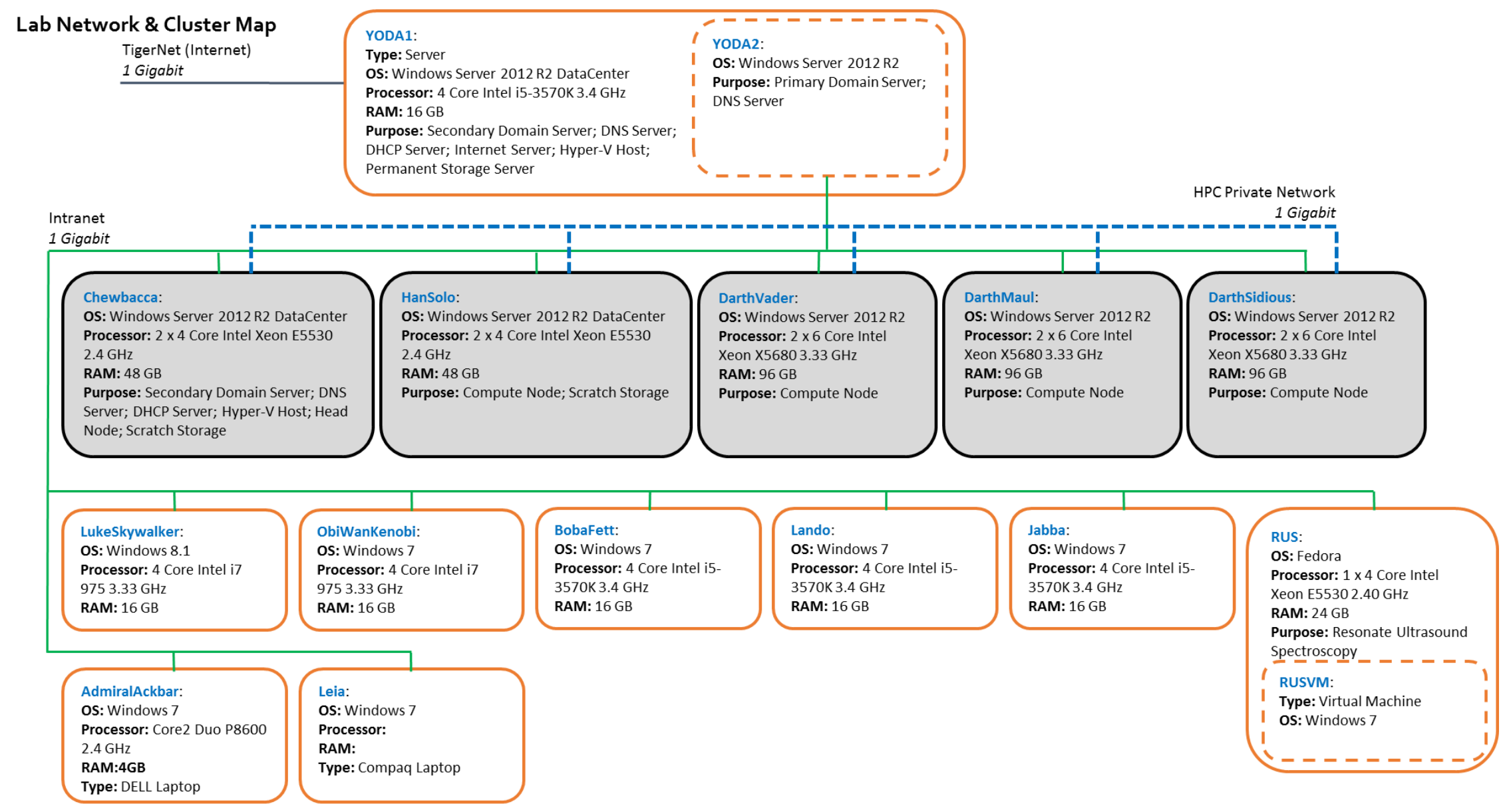

Updated January 2015

Figure 7.2. Detailed lab network and cluster map 


\section{2: Job Submission and Software}

Jobs are submitted to the HPC cluster through the HPC Job Scheduler shown in Figure

7.3. The scheduler allocates computational resources to allow running multiple jobs simultaneously. It also allows for jobs to be queued, thereby increasing utilization of cluster resources.

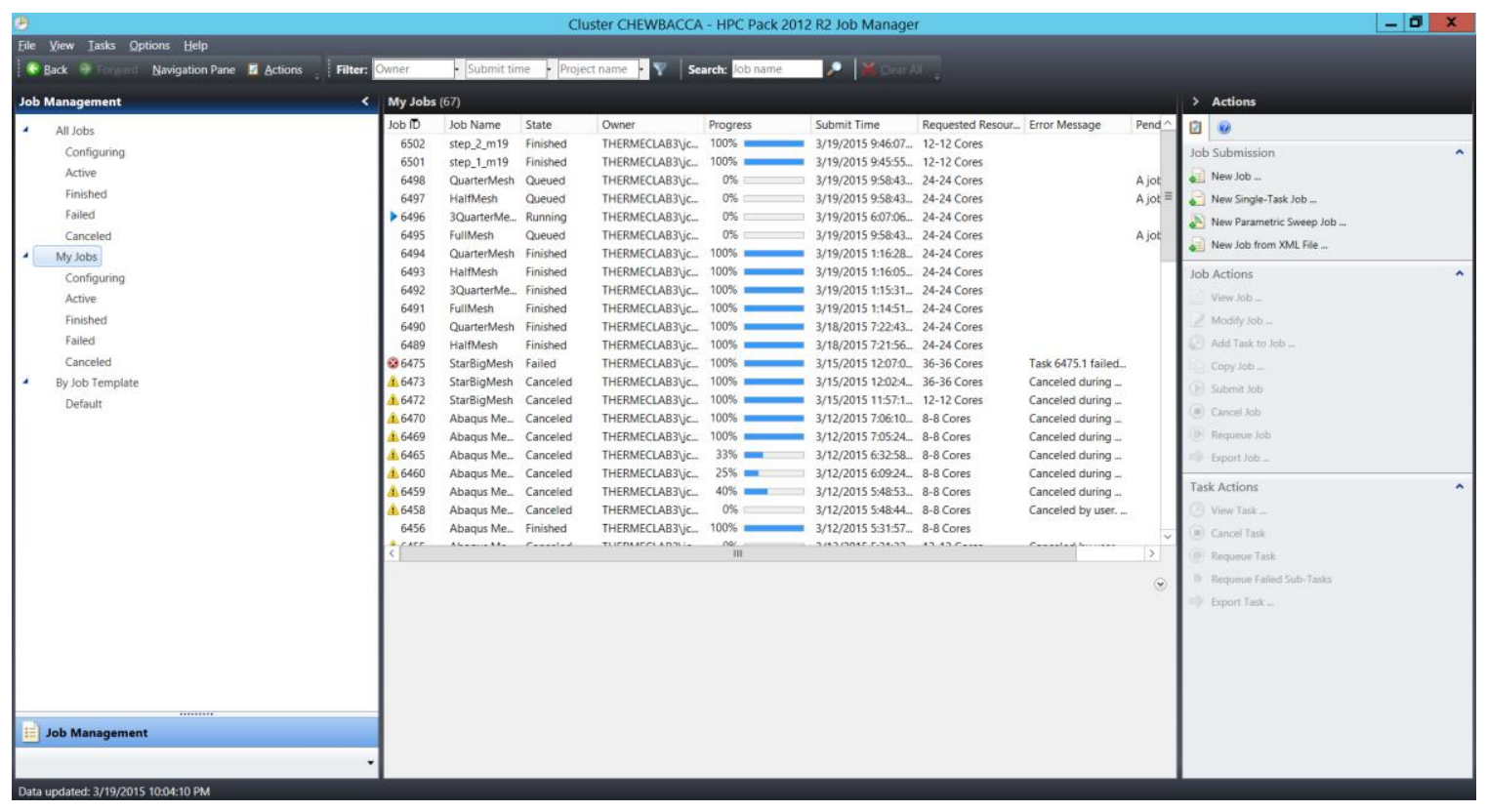

Figure 7.3. HPC job scheduler

When a new job is created, the user specifies a working directory, the command to execute, and the number of processors to run on. Typically, the execution command will load a particular file and program for analysis. For example, to submit a Star-CCM+ job, the execution command would be:

IIchewbacca\Apps $\backslash$ StarCCM+\starccm+ -mpi msmpi-batch simulation.sim 
This command runs Star-CCM+, using the Microsoft MPI implementation, in batch mode, and starts running the simulation.sim file. The simulation is should be set up ahead of time with some stopping criteria. When it finishes, the file is saved, Star-CCM+ closes, and the processors are released back to the scheduler.

With the lab computers all sharing a network, it is fairly simple to submit a job through the command line from any connected computer. To submit the same simulation referenced earlier through the command line rather than the GUI, the user would type the following into a command prompt:

job submit /scheduler:chewbacca /jobname:SimulationName /nodegroup:6CoreXeon /numcores:24 /stderr:ErrorFile.err/stdout:outFile.out /workdir: \Vhansolo\scratch WorkDir \lchewbaccalapps $\backslash$ StarCCM+\starccm+ mpi msmpi-batch simulation.sim

Similar commands exist for running other types of simulations (such as Abaqus).

Presently, Abaqus is limited to running on no more than 12 processor cores (one node).

However, this has yet to be a significant obstacle as the Star-CCM+ computations

typically require significantly greater resources. 


\section{CHAPTER 8: COMPUTATIONAL FLUID DYNAMICS (CFD)}

\section{1: Requirements for Mesh}

The geometry to be modeled in the CFD portion of the FSI simulations is based directly on the experiment geometry of Figure 4.2 and Figure 4.3. The presence of turbulent flow through a high-aspect ratio geometry presents unique challenges in meshing the fluid model. On one hand, the need to accurately capture the flow profile through a thin channel as well as the near wall shear stress solution requires a higher density mesh. On the other hand, in order for the simulations to run in a reasonable time on the available resources, a less dense mesh is required.

In order to develop an adequate mesh that is fine enough through the channel thickness without being too dense overall, it is necessary to anisotropically scale the cells. For the CFD models presented here, the fluid cells are stretched along the length and width of the channels, and shrunk through the channel thickness. Despite the geometry being a simple rectangular duct, the goal of a fine wall mesh and anisotropically scaled cells was somewhat difficult to achieve with the Star-CCM+ automated mesher. However, after numerous adjustments and custom settings on the individual surfaces, and adequate grid was created.

Recently, CD-Adapco has introduced the ability to generate fully structured meshes in Star-CCM+, but this capability was not scripted or utilized for these simulations. As this structured grid generator matures, it will allow grid generation similar to what is currently done in Abaqus, where scripts are generated to seed edges prior to meshing. This 
capability has proven invaluable in FEA modeling, and on occasion, a fluid grid has been created in Abaqus and exported to Star-CCM+. For the purposes of this study, the geometry was simple enough that grid generation could be completed in Star-CCM+ without significant difficulty.

When sizing the cells perpendicular to the wall, the $y+$ value at the centroid of the near wall cells must be considered in the context of the selected turbulent wall treatment. Low $y+$ wall treatments will resolve the viscous sublayer and require a y+ value less than five. High y+ wall treatments use wall functions to calculate the wall shear stress and require a $\mathrm{y}+$ value in the range of 35 100. An All $\mathrm{y}+$ treatment uses blended functions to provide a solution at any $\mathrm{y}+$ value, including the transition region.

\section{2: Turbulence and Mesh Validation Study}

The capability of a CFD code to accurately model flow development and pressure drop in wide, narrow ducts is the basis for this study. A variety of turbulence models and wall treatments have been applied to a thin-channel, high aspect ratio geometry with several fluid velocities. The following are the primary factors in determining the most appropriate turbulence model for this type of problem:

1) Accuracy of the pressure drop solution when compared to an analytic model

2) Accuracy of the wall shear stress solution in the fully developed region when compared to an analytic model

3) Accuracy of the near wall cell y+ calculation in the fully developed region when compared to an analytic model 
The chosen geometry is simple, thereby allowing an analytic solution with a high degree of accuracy. Within Star-CCM+, numerous different turbulence models and wall treatments are available. This study focuses primarily on two Reynolds Averaged Navier-Stokes (RANS) turbulence models: $k-\varepsilon$ and k- $\omega$. Within those models, multiple formulations are available, and within those formulations, multiple treatments for solving the near wall velocity gradient are available. Many of these wall treatments are dependent upon a specific range of $y+$ values for the near wall cell centroid. Therefore, the mesh for the geometry changes for a given wall treatment selection.

\subsection{1: Simplified Duct Geometry}

Figure 8.1 show the geometry of interest, with dimensions listed in the neighboring table. The boundary conditions include a constant velocity inlet and a constant zero-pressure outlet. 


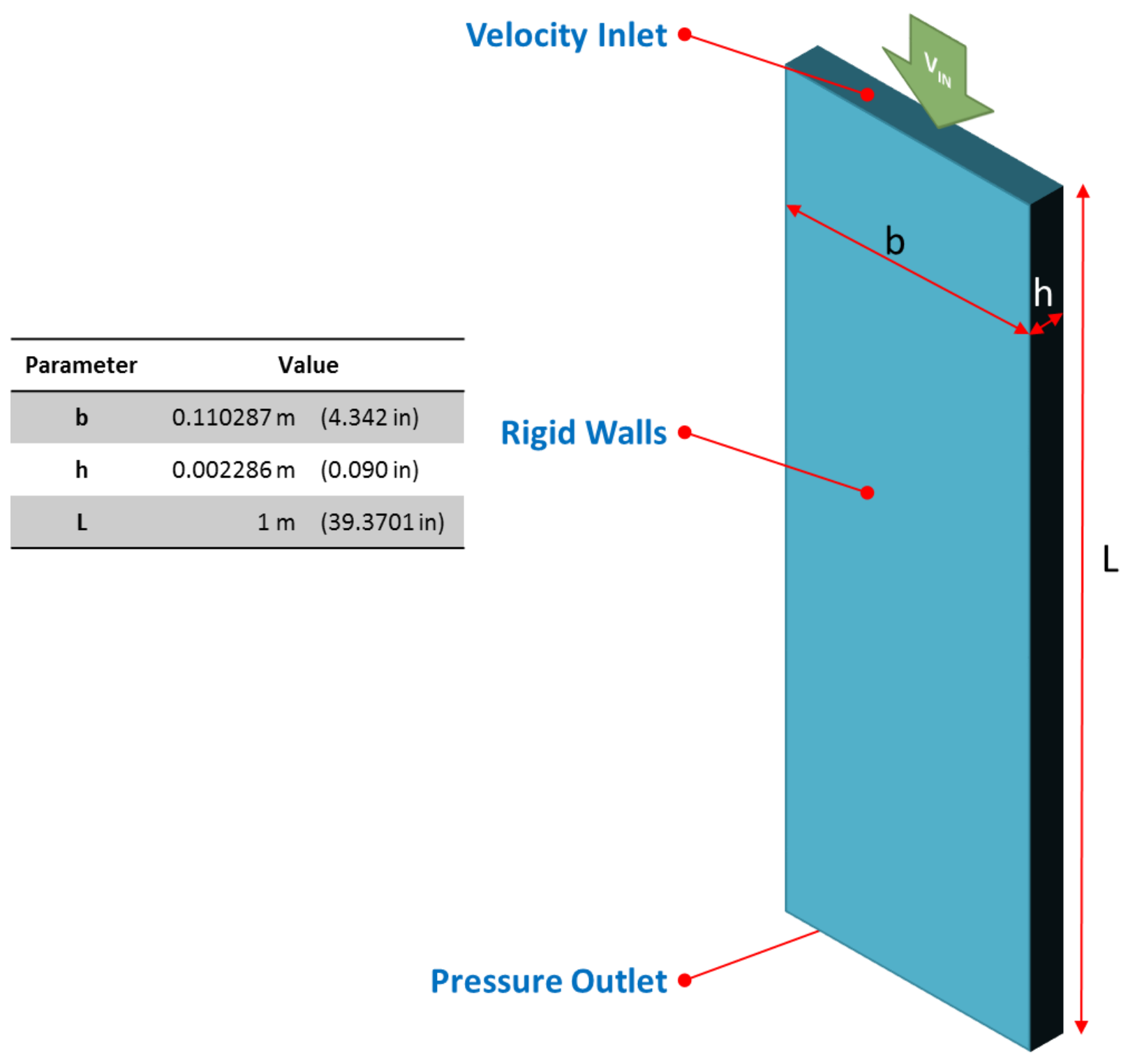

Figure 8.1. Fluid channel geometry

\subsection{2: Analytic Flow Solution}

The first metric for numeric model validation is the pressure drop through the channel.

Since the channel has a constant cross-section through the entire flow length, the only pressure losses will result from turbulent friction. 
Equations 2.15 through 2.18 provide the frictional pressure drop through the duct using the Colebrook equation [18]. Assuming smooth walls, Table 8.1 shows the calculation of pressure drop for the three velocities of interest.

Table 8.1. Analytic pressure drop calculations

\begin{tabular}{|c|c|c|c|c|}
\hline Parameter & & Value & & Units \\
\hline b & & 0.110287 & & $\mathrm{~m}$ \\
\hline h & & 0.002286 & & $\mathrm{~m}$ \\
\hline $\mathbf{L}$ & & 1 & & $\mathrm{~m}$ \\
\hline$\rho$ & & 997.561 & & $\mathrm{~kg} / \mathrm{m}^{3}$ \\
\hline$\mu$ & & 0.0008871 & & $\mathrm{~Pa}-\mathrm{s}$ \\
\hline $\mathbf{A}$ & & 0.000252116 & & $\mathrm{~m}^{2}$ \\
\hline $\mathbf{P}$ & & 0.225146 & & $\mathrm{~m}$ \\
\hline $\mathbf{D}_{\mathbf{H}}$ & & 0.004479157 & & $\mathrm{~m}$ \\
\hline$\overline{\mathbf{u}}$ & 2 & 5 & 9 & $\mathrm{~m} / \mathrm{s}$ \\
\hline $\operatorname{Re}$ & 10056 & 25139 & 45250 & \\
\hline $\mathbf{f}$ & 0.03084 & 0.02449 & 0.02136 & \\
\hline$\Delta \mathbf{P}$ & 13738.1 & 68167.4 & 192701.9 & $\mathrm{~Pa}$ \\
\hline
\end{tabular}

A second metric for numeric model validation is the wall shear stress in the fully developed region. Using Eq. 2.20, Table 8.2 shows these values at the velocities of interest.

Table 8.2. Analytic fully developed wall shear stress.

\begin{tabular}{ccccc}
\hline Parameter & \multicolumn{3}{c}{ Value } & Units \\
\hline$\overline{\mathbf{u}}$ & 2 & 5 & 9 & $\mathrm{~m} / \mathrm{s}$ \\
$\tau_{\mathbf{0}}$ & 15.38 & 76.33 & 215.79 & $\mathrm{~Pa}$ \\
\hline
\end{tabular}


With the shear stress calculation, it also possible to determine non-dimensional $\mathrm{y}^{+}$and $\mathrm{u}^{+}$ values in the fully developed region. The $\mathrm{y}^{+}$values in particular are useful in determining the appropriate near-wall grid size in the numeric models. Equations 2.26 through 2.29 provide the non-dimensional $\mathrm{u}^{+}$and $\mathrm{y}^{+}$values, as well as the wall distance, $\mathrm{y}$.

\subsection{3: Numeric Models}

Reynolds Averaged Navier-Stokes (RANS) turbulence models are the most commonly used due to their accuracy and computational efficiency. In this study, two different twoequation models are of interest: $k-\varepsilon$ and $k-\omega$. Within these models, multiple formulations are available in Star-CCM+.

The $\mathrm{k}-\varepsilon$ model solves for the turbulent kinetic energy, $\mathrm{k}$, and the turbulent dissipation per unit mass, $\varepsilon$. The two most common formulations are the standard and realizable. The realizable k- $\varepsilon$ model includes a number of improvements over the standard k- $\varepsilon$ model and more closely matches experimental data for a number of cases [47].

The $\mathrm{k}-\omega$ model solves for $\mathrm{k}$ and the turbulent dissipation per unit time, $\omega$. Generally, the standard Wilcox k- $\omega$ model provides better boundary layer solutions than the standard k$\varepsilon$ model [31] [32]. The shear stress transport (SST) Menter k- $\omega$ model provides two improvements to the Wilcox model. The SST model transitions to the standard k- $\varepsilon$ model in the outer boundary layer region and uses an alternate definition for the eddy-viscosity [33].

In near wall fluid flow, the flow gradient normal to the wall tends to develop on small scales, typically many orders of magnitude smaller than the geometry. When solving for 
the near wall flow in CFD, there are two options: either directly solve the flow in the viscous sub-layer and transition regions, or use an empirical correlation to estimate the wall shear stress.

While directly solving for the near wall flow may provide a more accurate solution, it also requires considerable grid refinement, and therefore longer runtimes. Resolving the viscous sub-layer requires $y+$ values for the near wall cells of less than 5, and ideally closer to 1 . To solve for the viscous sub-layer on a well-refined grid, a low y+ wall treatment is necessary.

If an empirical estimation is adequate, a wall treatment function can estimate the wall shear stress based on correlations developed from experiments. The y+ values of the near wall cells typically need to be between approximately 35 and 100 . Since there is no need to resolve the viscous sub-layer or transition flow, these functions utilize much coarser grids, and therefore have substantially shorter runtimes. This advantage comes with the potential for less detailed results. These empirical solutions are accessed through the high $y+$ wall treatment solvers.

Hybrid approaches are also available which attempt to switch between the low and high $y+$ treatments. Some of these solvers use blended functions and are accessed as All y+ wall treatments. Others use a Two-Layer approach and split the flow between Low y+ and High $y+$ options at some predetermined $y+$ value. These functions may struggle with wall flows in the transition region between the viscous sub-layer flow and fully turbulent regions. 
Each turbulence model has different capabilities in terms of the available wall functions. An attempt has been made to study the applicable wall functions for each turbulence model of interest. While a large number of combinations were studied, this is far from an exhaustive list of permutations.

Since the validity of a given wall function is dependent on the grid (particularly, the thickness of the near wall cells), three grids were selected for this study. A 'Coarse' mesh was designed for a near wall $y+$ value of approximately 35 with a fluid velocity of $2 \mathrm{~m} / \mathrm{s}$. This should yield higher $\mathrm{y}+$ values at $5 \mathrm{~m} / \mathrm{s}$ and $9 \mathrm{~m} / \mathrm{s}$. As shown in Figure 8.2, the mesh is quite coarse, with only 5 elements through the thickness of the channel. While this may seem excessively coarse, should the High y+ and All y+ wall functions provide adequate results with this mesh, the resulting low runtimes will be highly advantageous. 


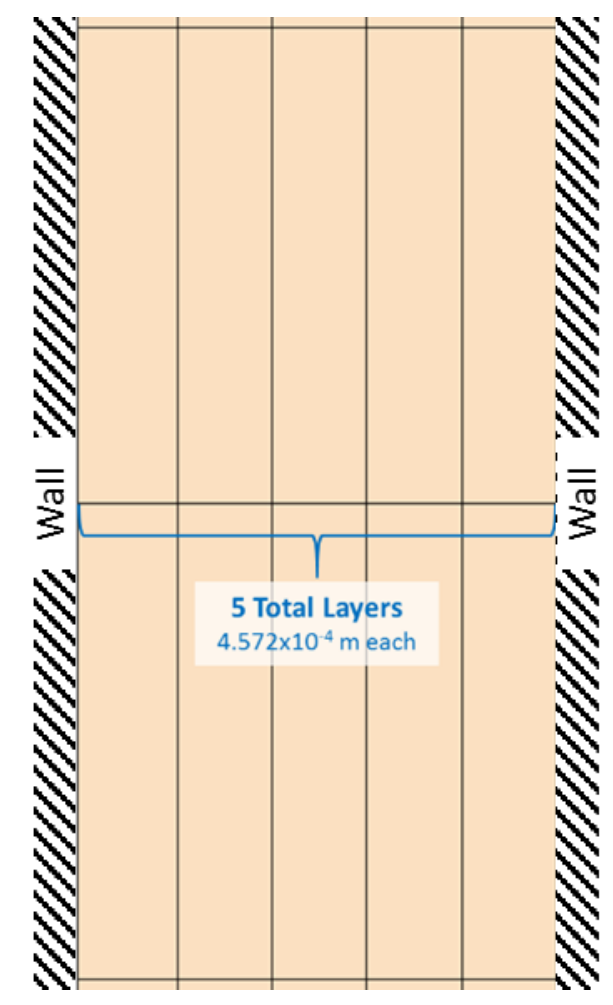

Figure 8.2. Cross section of coarse mesh

A 'Medium' mesh is designed to provide higher density through the channel thickness, without including a highly resolved near wall mesh. Since the near wall y+ values for this mesh will tend to be in the transition region, the mesh shown in Figure 8.3 will help assess the accuracy of wall functions in the transition region. 


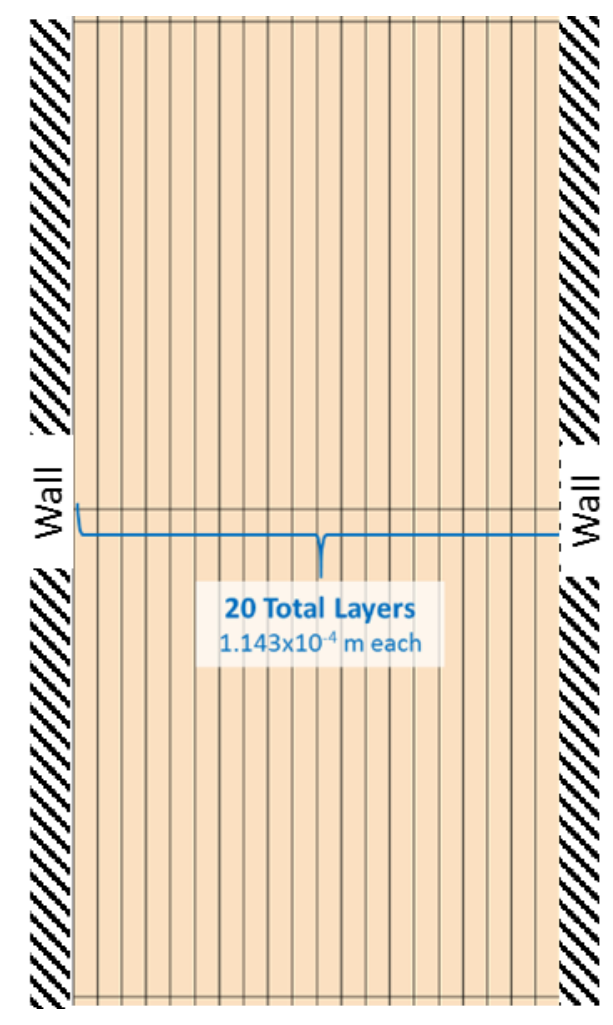

Figure 8.3. Cross section of medium mesh

Finally, a third mesh has been resolved near the wall to allow for directly solving the viscous sub-layer. This mesh can utilize Low y+ wall functions, and is targeted at near wall $y+$ values of 1 at $9 \mathrm{~m} / \mathrm{s}$. The $y+$ values will be less than 1 at $5 \mathrm{~m} / \mathrm{s}$ and $2 \mathrm{~m} / \mathrm{s}$. While substantially longer runtimes are expected, the solution should be more accurate.

Additionally, given how thin the fluid channel is, the ability to resolve the viscous sublayer and directly solve for the wall shear stress may override concerns about excessive runtimes. 


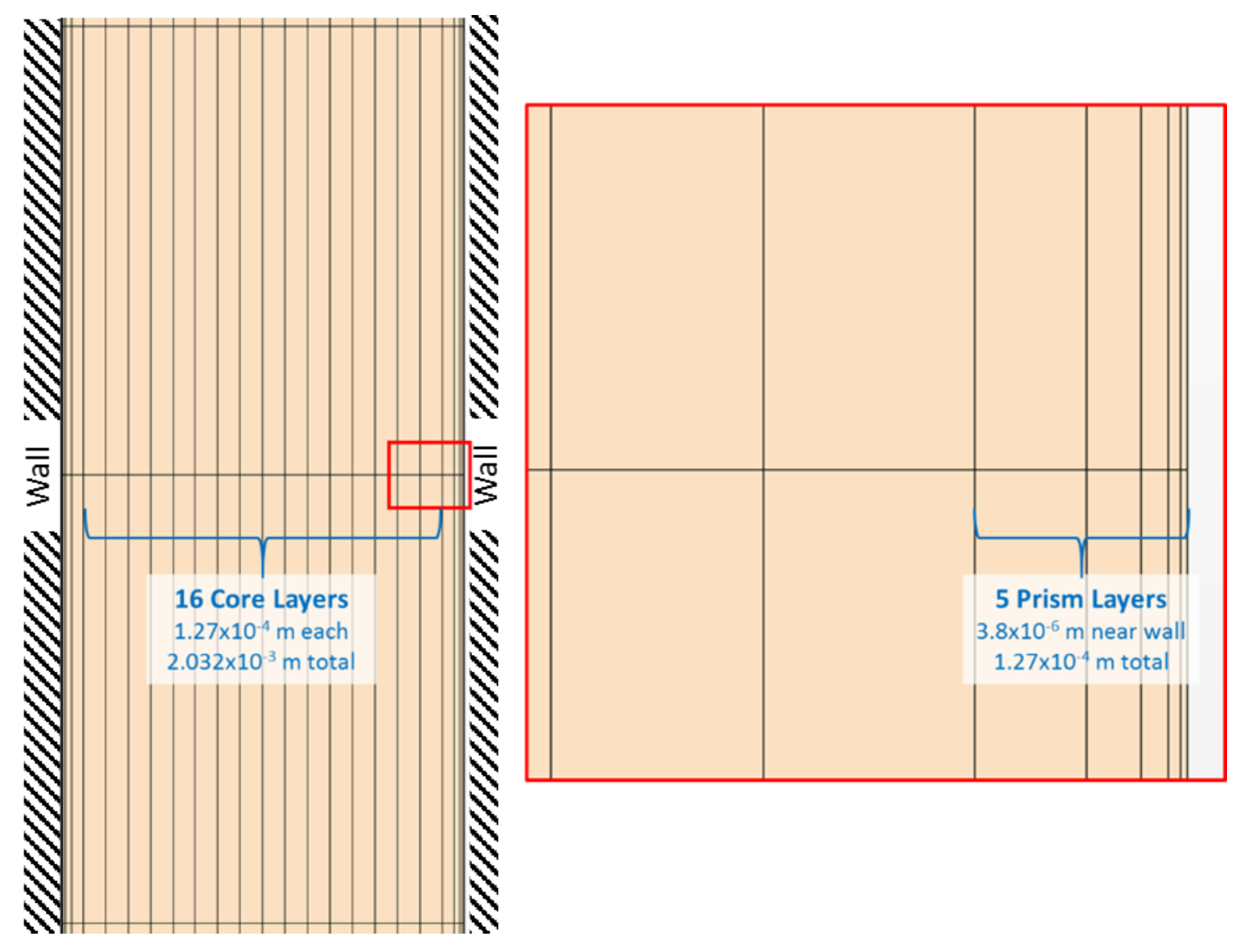

Figure 8.4. Cross section of fine mesh with close-up of near wall mesh

All three meshes utilize anisotropic cells - the width and length dimensions are nearly square and much larger than the thickness dimension. Table 8.3 provides the length, width, and thickness dimensions for the core and near wall cells of all three meshes.

Table 8.3. Mesh dimensions

\begin{tabular}{cccccc}
\hline & Width $(\mathbf{m})$ & Length $(\mathbf{m})$ & Core Thickness $(\mathbf{m})$ & $\begin{array}{c}\text { Near Wall } \\
\text { Thickness }(\mathbf{m})\end{array}$ & Total Cells \\
\hline Coarse & $2.2635 \times 10^{-3}$ & $2.2635 \times 10^{-3}$ & $4.572 \times 10^{-4}$ & $4.572 \times 10^{-4}$ & 108290 \\
Medium & $2.286 \times 10^{-3}$ & $2.286 \times 10^{-3}$ & $1.143 \times 10^{-4}$ & $1.143 \times 10^{-4}$ & 420480 \\
Fine & $2.54 \times 10^{-3}$ & $2.54 \times 10^{-3}$ & $1.27 \times 10^{-4}$ & $3.8 \times 10^{-6}$ & 450736 \\
\hline
\end{tabular}


Using eqns. 2.26 through 2.29, along with the near wall cell thicknesses in Table 8.3, it is possible to calculate the wall $y+$ values. Note that these values, shown in Table 8.4 , use $1 / 2$ the wall cell thickness in order to determine the $y+$ values at the cell centroid.

Table 8.4. Analytic near wall y+ values in the fully developed region

\begin{tabular}{cccc}
\hline & $\mathbf{2} \mathbf{~} / \mathbf{s}$ & $\mathbf{5 ~} \mathbf{~} / \mathbf{s}$ & $\mathbf{9 ~} \mathbf{~} / \mathbf{s}$ \\
\hline Coarse & 31.87 & 70.98 & 119.34 \\
Medium & 7.97 & 17.75 & 29.84 \\
Fine & 0.26 & 0.59 & 0.99 \\
\hline
\end{tabular}

\subsection{4: Results}

Table 8.5 through Table 8.8 show the pressure drop results for each turbulence model. Within each table, the pressure drop for each inlet velocity, wall treatment, and mesh combination is provided. Additionally, the percent difference from the analytic solution (duplicated at the top of each table) is provided below the calculated Star-CCM+ pressure drop value. 
Table 8.5. Standard k- $\varepsilon$ pressure drop results

\begin{tabular}{|c|c|c|c|c|c|}
\hline $\begin{array}{c}\text { Wall } \\
\text { Treatment }\end{array}$ & Mesh & & $2 \mathrm{~m} / \mathrm{s}$ & $5 \mathrm{~m} / \mathrm{s}$ & $9 \mathrm{~m} / \mathrm{s}$ \\
\hline \multicolumn{2}{|c|}{ Analytic } & & $13738.1 \mathrm{~Pa}$ & $68167.4 \mathrm{~Pa}$ & $192701.9 \mathrm{~Pa}$ \\
\hline \multirow{2}{*}{ High y+ } & \multirow{2}{*}{ Coarse } & Pressure & $14755.2 \mathrm{~Pa}$ & $72662.1 \mathrm{~Pa}$ & $204696.6 \mathrm{~Pa}$ \\
\hline & & $\%$ Difference & $7.40 \%$ & $6.59 \%$ & $6.22 \%$ \\
\hline \multirow{6}{*}{$\begin{array}{c}\text { Two-Layer } \\
\text { All y+ }\end{array}$} & \multirow{2}{*}{ Coarse } & Pressure & $14905.9 \mathrm{~Pa}$ & $72026.1 \mathrm{~Pa}$ & $203639.2 \mathrm{~Pa}$ \\
\hline & & $\%$ Difference & $8.50 \%$ & $5.66 \%$ & $5.68 \%$ \\
\hline & \multirow{2}{*}{ Medium } & Pressure & $18396.2 \mathrm{~Pa}$ & $76283.8 \mathrm{~Pa}$ & $204367.7 \mathrm{~Pa}$ \\
\hline & & $\%$ Difference & $33.91 \%$ & $11.91 \%$ & $6.05 \%$ \\
\hline & \multirow{2}{*}{ Fine } & Pressure & $17485.3 \mathrm{~Pa}$ & $78444.3 \mathrm{~Pa}$ & $221673.2 \mathrm{~Pa}$ \\
\hline & & $\%$ Difference & $27.28 \%$ & $15.08 \%$ & $15.03 \%$ \\
\hline \multirow{6}{*}{$\begin{array}{c}\text { Low-Re } \\
\text { All y+ }\end{array}$} & \multirow{2}{*}{ Coarse } & Pressure & $14890.4 \mathrm{~Pa}$ & $72006.9 \mathrm{~Pa}$ & $203572.9 \mathrm{~Pa}$ \\
\hline & & $\%$ Difference & $8.39 \%$ & $5.63 \%$ & $5.64 \%$ \\
\hline & \multirow{2}{*}{ Medium } & Pressure & $19205.2 \mathrm{~Pa}$ & $78419.0 \mathrm{~Pa}$ & $206568.5 \mathrm{~Pa}$ \\
\hline & & $\%$ Difference & $39.79 \%$ & $15.04 \%$ & $7.20 \%$ \\
\hline & \multirow{2}{*}{ Fine } & Pressure & $18476.4 \mathrm{~Pa}$ & $97812.8 \mathrm{~Pa}$ & $273647.6 \mathrm{~Pa}$ \\
\hline & & $\%$ Difference & $34.49 \%$ & $43.49 \%$ & $42.01 \%$ \\
\hline \multirow{2}{*}{$\begin{array}{l}\text { Low-Re } \\
\text { Low y+ }\end{array}$} & \multirow{2}{*}{ Fine } & Pressure & $26107.8 \mathrm{~Pa}$ & $139863.1 \mathrm{~Pa}$ & $383257.7 \mathrm{~Pa}$ \\
\hline & & $\%$ Difference & $90.04 \%$ & $105.18 \%$ & $98.89 \%$ \\
\hline
\end{tabular}


Table 8.6. Realizable k- $\varepsilon$ pressure drop results

\begin{tabular}{|c|c|c|c|c|c|}
\hline $\begin{array}{c}\text { Wall } \\
\text { Treatment }\end{array}$ & Mesh & & $2 \mathrm{~m} / \mathrm{s}$ & $5 \mathrm{~m} / \mathrm{s}$ & $9 \mathrm{~m} / \mathrm{s}$ \\
\hline \multicolumn{2}{|c|}{ Analytic } & & $13738.1 \mathrm{~Pa}$ & $68167.4 \mathrm{~Pa}$ & $192701.9 \mathrm{~Pa}$ \\
\hline \multirow{2}{*}{ High y+ } & \multirow{2}{*}{ Coarse } & Pressure & $14586.4 \mathrm{~Pa}$ & $71858.2 \mathrm{~Pa}$ & $202650.1 \mathrm{~Pa}$ \\
\hline & & $\%$ Difference & $6.17 \%$ & $5.41 \%$ & $5.16 \%$ \\
\hline \multirow{7}{*}{$\begin{array}{c}\text { Two-Layer } \\
\text { All y+ }\end{array}$} & \multirow{2}{*}{ Coarse } & Pressure & $14412.0 \mathrm{~Pa}$ & $70772.2 \mathrm{~Pa}$ & $201053.0 \mathrm{~Pa}$ \\
\hline & & $\%$ Difference & $4.91 \%$ & $3.82 \%$ & $4.33 \%$ \\
\hline & \multirow{2}{*}{ Medium } & Pressure & $16084.4 \mathrm{~Pa}$ & $68621.4 \mathrm{~Pa}$ & $191053.6 \mathrm{~Pa}$ \\
\hline & & $\%$ Difference & $17.08 \%$ & $0.67 \%$ & $-0.86 \%$ \\
\hline & \multirow{3}{*}{ Fine } & Pressure & $15869.8 \mathrm{~Pa}$ & 74098.6 Pa & $201283.7 \mathrm{~Pa}$ \\
\hline & & & & & \\
\hline & & $\%$ Difference & $15.52 \%$ & $8.70 \%$ & $4.45 \%$ \\
\hline
\end{tabular}

Table 8.7. Standard (Wilcox) k-w pressure drop results

\begin{tabular}{|c|c|c|c|c|c|}
\hline $\begin{array}{c}\text { Wall } \\
\text { Treatment }\end{array}$ & Mesh & & $2 \mathrm{~m} / \mathrm{s}$ & $5 \mathrm{~m} / \mathrm{s}$ & $9 \mathrm{~m} / \mathrm{s}$ \\
\hline \multicolumn{2}{|c|}{ Analytic } & & $13738.1 \mathrm{~Pa}$ & $68167.4 \mathrm{~Pa}$ & $192701.9 \mathrm{~Pa}$ \\
\hline \multirow{2}{*}{ High y+ } & \multirow{2}{*}{ Coarse } & Pressure & $15270.5 \mathrm{~Pa}$ & $75157.4 \mathrm{~Pa}$ & $211548.5 \mathrm{~Pa}$ \\
\hline & & $\%$ Difference & $11.15 \%$ & $10.25 \%$ & $9.78 \%$ \\
\hline \multirow{6}{*}{ All y+ } & \multirow{2}{*}{ Coarse } & Pressure & $15627.1 \mathrm{~Pa}$ & $74713.3 \mathrm{~Pa}$ & $210644.3 \mathrm{~Pa}$ \\
\hline & & $\%$ Difference & $13.75 \%$ & $9.60 \%$ & $9.31 \%$ \\
\hline & \multirow{2}{*}{ Medium } & Pressure & $20844.7 \mathrm{~Pa}$ & 83979.6 Pa & $218798.9 \mathrm{~Pa}$ \\
\hline & & $\%$ Difference & $51.73 \%$ & $23.20 \%$ & $13.54 \%$ \\
\hline & \multirow{2}{*}{ Fine } & Pressure & $14748.6 \mathrm{~Pa}$ & $67615.4 \mathrm{~Pa}$ & $187130.4 \mathrm{~Pa}$ \\
\hline & & $\%$ Difference & $7.36 \%$ & $-0.81 \%$ & $-2.89 \%$ \\
\hline \multirow{2}{*}{ Low y+ } & \multirow{2}{*}{ Fine } & Pressure & $14779.6 \mathrm{~Pa}$ & $68809.3 \mathrm{~Pa}$ & $192121.8 \mathrm{~Pa}$ \\
\hline & & of Difference & $7580 \%$ & $0040 \%$ & $30 \%$ \\
\hline
\end{tabular}


Table 8.8. SST (Menter) k-w pressure drop results

\begin{tabular}{|c|c|c|c|c|c|}
\hline $\begin{array}{c}\text { Wall } \\
\text { Treatment }\end{array}$ & Mesh & & $2 \mathrm{~m} / \mathrm{s}$ & $5 \mathrm{~m} / \mathrm{s}$ & $9 \mathrm{~m} / \mathrm{s}$ \\
\hline \multicolumn{2}{|c|}{ Analytic } & & $13738.1 \mathrm{~Pa}$ & $68167.4 \mathrm{~Pa}$ & $192701.9 \mathrm{~Pa}$ \\
\hline \multirow{2}{*}{ High y+ } & \multirow{2}{*}{ Coarse } & & $15266.3 \mathrm{~Pa}$ & $75004.3 \mathrm{~Pa}$ & $210935.4 \mathrm{~Pa}$ \\
\hline & & $\%$ Difference & $11.12 \%$ & $10.03 \%$ & $9.46 \%$ \\
\hline \multirow{6}{*}{ All y+ } & \multirow{2}{*}{ Coarse } & Pressure & $15638.6 \mathrm{~Pa}$ & $74582.8 \mathrm{~Pa}$ & $210098.1 \mathrm{~Pa}$ \\
\hline & & $\%$ Difference & $13.83 \%$ & $9.41 \%$ & $9.03 \%$ \\
\hline & \multirow{2}{*}{ Medium } & Pressure & $20437.7 \mathrm{~Pa}$ & $81272.0 \mathrm{~Pa}$ & $214707.4 \mathrm{~Pa}$ \\
\hline & & $\%$ Difference & $48.77 \%$ & $19.22 \%$ & $11.42 \%$ \\
\hline & \multirow{2}{*}{ Fine } & Pressure & $14185.9 \mathrm{~Pa}$ & $66281.4 \mathrm{~Pa}$ & $176916.8 \mathrm{~Pa}$ \\
\hline & & $\%$ Difference & $3.26 \%$ & $-2.77 \%$ & $-8.19 \%$ \\
\hline \multirow{2}{*}{ Low y+ } & \multirow{2}{*}{ Fine } & Pressure & $14185.8 \mathrm{~Pa}$ & $67167.0 \mathrm{~Pa}$ & $180881.4 \mathrm{~Pa}$ \\
\hline & & $\%$ Difference & $3.26 \%$ & $-1.47 \%$ & $-6.13 \%$ \\
\hline
\end{tabular}

For a given model, it is possible to calculate the root mean square of the three percent differences for each velocity. This resulting value is effectively an average difference from the analytic solution for that particular turbulence model, wall treatment, and mesh combination. Using the data in the preceding tables, this calculation was completed for every model, with the results shown in Figure 8.5. 
Pressure Drop RMS Error

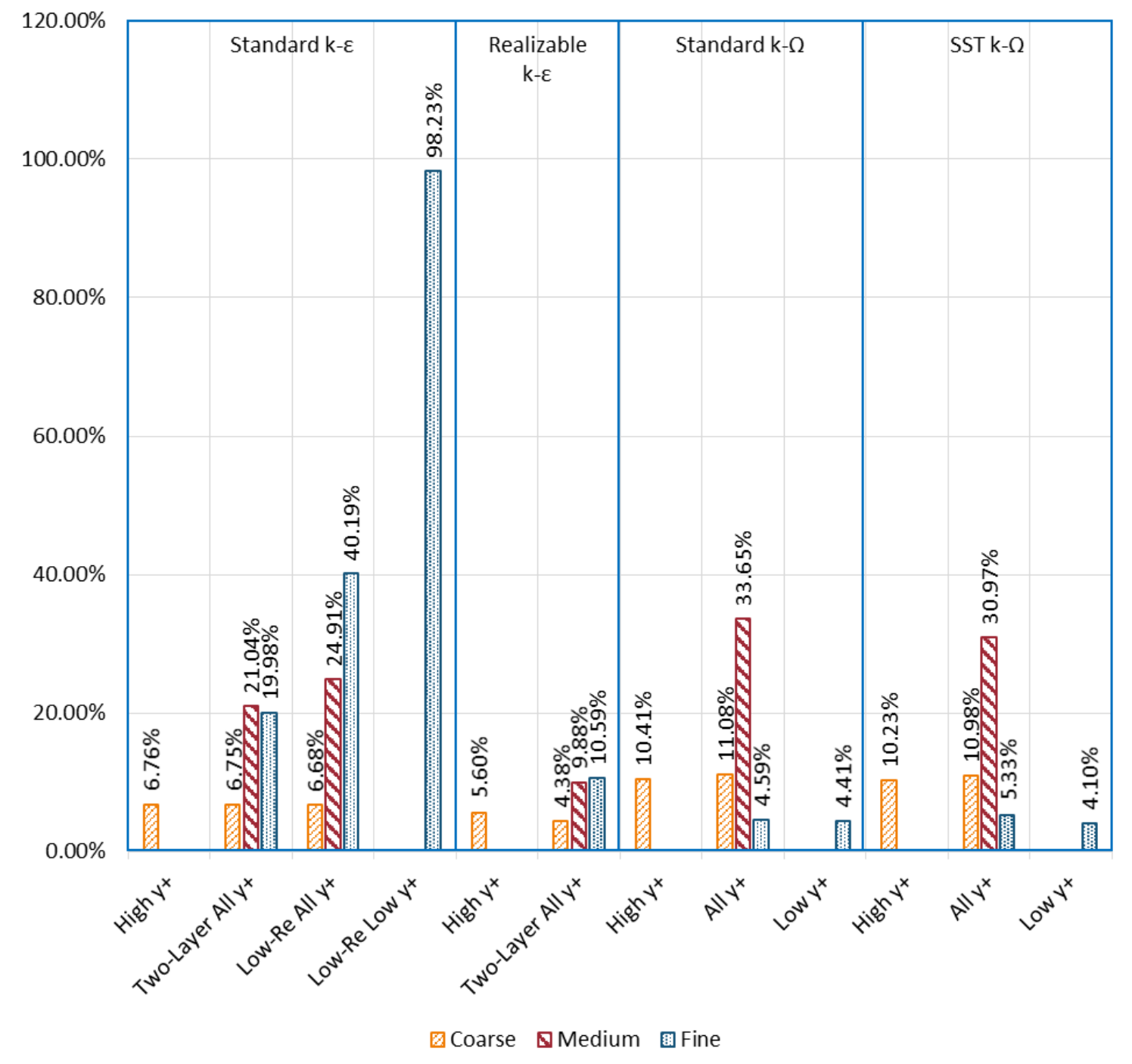

Figure 8.5. RMS pressure drop error for all models

From these results, both the standard Wilcox and SST Menter k- $\omega$ models with fine meshes and either All y+ or Low y+ wall treatments provided the closest match to the

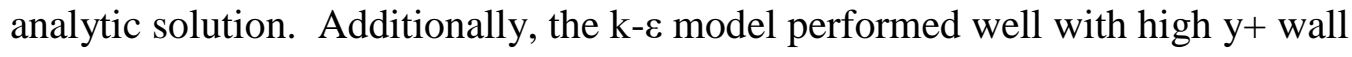
treatments and a coarse mesh. 
Table 8.9 through Table 8.12 show the wall shear stress results for each turbulence model. Within each table, the wall shear stress for each inlet velocity, wall treatment, and mesh combination is provided. Additionally, the percent difference from the analytic solution (duplicated at the top of each table) is provided below the calculated Star-CCM+ wall shear stress value.

Table 8.9. Standard k- $\varepsilon$ wall shear stress results

\begin{tabular}{|c|c|c|c|c|c|}
\hline $\begin{array}{c}\text { Wall } \\
\text { Treatment }\end{array}$ & Mesh & & $2 \mathrm{~m} / \mathrm{s}$ & $5 \mathrm{~m} / \mathrm{s}$ & $9 \mathrm{~m} / \mathrm{s}$ \\
\hline \multicolumn{2}{|c|}{ Analytic } & & $15.38 \mathrm{~Pa}$ & $76.33 \mathrm{~Pa}$ & $215.79 \mathrm{~Pa}$ \\
\hline \multirow{2}{*}{ High y+ } & \multirow{2}{*}{ Coarse } & Pressure & $16.77 \mathrm{~Pa}$ & $82.60 \mathrm{~Pa}$ & $232.70 \mathrm{~Pa}$ \\
\hline & & $\%$ Difference & $9.04 \%$ & $8.21 \%$ & $7.84 \%$ \\
\hline \multirow{6}{*}{$\begin{array}{c}\text { Two-Layer } \\
\text { All y+ }\end{array}$} & \multirow{2}{*}{ Coarse } & Pressure & $16.98 \mathrm{~Pa}$ & $81.89 \mathrm{~Pa}$ & $231.51 \mathrm{~Pa}$ \\
\hline & & $\%$ Difference & $10.37 \%$ & $7.28 \%$ & $7.29 \%$ \\
\hline & \multirow{2}{*}{$\begin{array}{l}\text { Mediu } \\
\text { m }\end{array}$} & Pressure & $20.82 \mathrm{~Pa}$ & $86.29 \mathrm{~Pa}$ & $231.04 \mathrm{~Pa}$ \\
\hline & & $\%$ Difference & $35.36 \%$ & $13.04 \%$ & $7.07 \%$ \\
\hline & \multirow{2}{*}{ Fine } & Pressure & $19.54 \mathrm{~Pa}$ & $83.32 \mathrm{~Pa}$ & $251.00 \mathrm{~Pa}$ \\
\hline & & $\%$ Difference & $27.00 \%$ & $9.15 \%$ & $16.32 \%$ \\
\hline \multirow{6}{*}{$\begin{array}{c}\text { Low-Re } \\
\text { All y+ }\end{array}$} & \multirow{2}{*}{ Coarse } & Pressure & $16.89 \mathrm{~Pa}$ & $81.87 \mathrm{~Pa}$ & $231.46 \mathrm{~Pa}$ \\
\hline & & $\%$ Difference & $9.77 \%$ & $7.25 \%$ & $7.26 \%$ \\
\hline & \multirow{2}{*}{$\underset{\mathrm{m}}{\text { Mediu }}$} & Pressure & $21.74 \mathrm{~Pa}$ & $88.63 \mathrm{~Pa}$ & $233.32 \mathrm{~Pa}$ \\
\hline & & $\%$ Difference & $41.30 \%$ & $16.10 \%$ & $8.12 \%$ \\
\hline & \multirow{2}{*}{ Fine } & Pressure & $22.34 \mathrm{~Pa}$ & $123.68 \mathrm{~Pa}$ & $327.00 \mathrm{~Pa}$ \\
\hline & & $\%$ Difference & $45.24 \%$ & $62.03 \%$ & $51.54 \%$ \\
\hline \multirow{2}{*}{$\begin{array}{l}\text { Low-Re } \\
\text { Low y+ }\end{array}$} & \multirow{2}{*}{ Fine } & Pressure & $27.65 \mathrm{~Pa}$ & $118.88 \mathrm{~Pa}$ & $362.53 \mathrm{~Pa}$ \\
\hline & & $\%$ Difference & $79.74 \%$ & $55.73 \%$ & $68.01 \%$ \\
\hline
\end{tabular}


Table 8.10. Realizable k- $\varepsilon$ wall shear stress results

\begin{tabular}{|c|c|c|c|c|c|}
\hline $\begin{array}{c}\text { Wall } \\
\text { Treatment }\end{array}$ & Mesh & & $2 \mathrm{~m} / \mathrm{s}$ & $5 \mathrm{~m} / \mathrm{s}$ & $9 \mathrm{~m} / \mathrm{s}$ \\
\hline \multicolumn{2}{|c|}{ Analytic } & & $15.38 \mathrm{~Pa}$ & $76.33 \mathrm{~Pa}$ & $215.79 \mathrm{~Pa}$ \\
\hline \multirow{2}{*}{ High y+ } & \multirow{2}{*}{ Coarse } & Pressure & $16.56 \mathrm{~Pa}$ & $81.58 \mathrm{~Pa}$ & $230.06 \mathrm{~Pa}$ \\
\hline & & $\%$ Difference & $7.63 \%$ & $6.87 \%$ & $6.62 \%$ \\
\hline \multirow{7}{*}{$\begin{array}{c}\text { Two-Layer } \\
\text { All y+ }\end{array}$} & \multirow{2}{*}{ Coarse } & Pressure & $16.39 \mathrm{~Pa}$ & $80.38 \mathrm{~Pa}$ & $228.27 \mathrm{~Pa}$ \\
\hline & & $\%$ Difference & $6.57 \%$ & $5.30 \%$ & $5.78 \%$ \\
\hline & \multirow{2}{*}{ Medium } & Pressure & $18.58 \mathrm{~Pa}$ & $76.99 \mathrm{~Pa}$ & $214.89 \mathrm{~Pa}$ \\
\hline & & $\%$ Difference & $20.76 \%$ & $0.86 \%$ & $-0.42 \%$ \\
\hline & \multirow{3}{*}{ Fine } & Pressure & $17.75 \mathrm{~Pa}$ & $82.43 \mathrm{~Pa}$ & $233.00 \mathrm{~Pa}$ \\
\hline & & & & & \\
\hline & & $\%$ Difference & $15.39 \%$ & $7.99 \%$ & $7.98 \%$ \\
\hline
\end{tabular}

Table 8.11. Standard (Wilcox) k- $\omega$ wall shear stress results

\begin{tabular}{|c|c|c|c|c|c|}
\hline $\begin{array}{c}\text { Wall } \\
\text { Treatment }\end{array}$ & Mesh & & $2 \mathrm{~m} / \mathrm{s}$ & $5 \mathrm{~m} / \mathrm{s}$ & $9 \mathrm{~m} / \mathrm{s}$ \\
\hline \multicolumn{2}{|c|}{ Analytic } & & $15.38 \mathrm{~Pa}$ & $76.33 \mathrm{~Pa}$ & $215.79 \mathrm{~Pa}$ \\
\hline \multirow{2}{*}{ High y+ } & \multirow{2}{*}{ Coarse } & Pressure & $17.35 \mathrm{~Pa}$ & $85.43 \mathrm{~Pa}$ & $240.52 \mathrm{~Pa}$ \\
\hline & & $\%$ Difference & $12.81 \%$ & $11.92 \%$ & $11.46 \%$ \\
\hline \multirow{7}{*}{ All y+ } & \multirow{2}{*}{ Coarse } & Pressure & $17.70 \mathrm{~Pa}$ & $84.90 \mathrm{~Pa}$ & $239.51 \mathrm{~Pa}$ \\
\hline & & $\%$ Difference & $15.08 \%$ & $11.22 \%$ & $10.99 \%$ \\
\hline & \multirow{3}{*}{ Medium } & Pressure & $23.59 \mathrm{~Pa}$ & $94.90 \mathrm{~Pa}$ & $247.29 \mathrm{~Pa}$ \\
\hline & & $m^{\prime} \cdot c$ & & & \\
\hline & & $\%$ Difference & $53.37 \%$ & $24.33 \%$ & $14.60 \%$ \\
\hline & \multirow{2}{*}{ Fine } & Pressure & $16.55 \mathrm{~Pa}$ & $75.85 \mathrm{~Pa}$ & $210.08 \mathrm{~Pa}$ \\
\hline & & $\%$ Difference & $7.57 \%$ & $-0.64 \%$ & $-2.65 \%$ \\
\hline \multirow{2}{*}{ Low y+ } & \multirow{2}{*}{ Fine } & Pressure & $16.56 \mathrm{~Pa}$ & $76.51 \mathrm{~Pa}$ & $212.69 \mathrm{~Pa}$ \\
\hline & & $\%$ Difference & $7.63 \%$ & $0.24 \%$ & $-1.43 \%$ \\
\hline
\end{tabular}


Table 8.12. SST (Menter) k- $\omega$ wall shear stress results

\begin{tabular}{|c|c|c|c|c|c|}
\hline $\begin{array}{c}\text { Wall } \\
\text { Treatment }\end{array}$ & Mesh & & $2 \mathrm{~m} / \mathrm{s}$ & $5 \mathrm{~m} / \mathrm{s}$ & $9 \mathrm{~m} / \mathrm{s}$ \\
\hline \multicolumn{2}{|c|}{ Analytic } & & $15.38 \mathrm{~Pa}$ & $76.33 \mathrm{~Pa}$ & $215.79 \mathrm{~Pa}$ \\
\hline \multirow{2}{*}{ High y+ } & \multirow{2}{*}{ Coarse } & Pressure & $17.39 \mathrm{~Pa}$ & $85.47 \mathrm{~Pa}$ & $240.44 \mathrm{~Pa}$ \\
\hline & & $\%$ Difference & $13.03 \%$ & $11.97 \%$ & $11.43 \%$ \\
\hline \multirow{6}{*}{ All y+ } & \multirow{2}{*}{ Coarse } & Pressure & $17.75 \mathrm{~Pa}$ & $84.93 \mathrm{~Pa}$ & $239.41 \mathrm{~Pa}$ \\
\hline & & $\%$ Difference & $15.37 \%$ & $11.27 \%$ & $10.95 \%$ \\
\hline & \multirow{2}{*}{ Medium } & Pressure & $23.18 \mathrm{~Pa}$ & $91.96 \mathrm{~Pa}$ & $243.10 \mathrm{~Pa}$ \\
\hline & & $\%$ Difference & $50.67 \%$ & $20.47 \%$ & $12.66 \%$ \\
\hline & \multirow{2}{*}{ Fine } & Pressure & $15.94 \mathrm{~Pa}$ & $74.59 \mathrm{~Pa}$ & $199.00 \mathrm{~Pa}$ \\
\hline & & $\%$ Difference & $3.60 \%$ & $-2.29 \%$ & $-7.78 \%$ \\
\hline \multirow{2}{*}{ Low y+ } & \multirow{2}{*}{ Fine } & Pressure & $15.92 \mathrm{~Pa}$ & $75.04 \mathrm{~Pa}$ & $201.08 \mathrm{~Pa}$ \\
\hline & & $\%$ Difference & $3.50 \%$ & $-1.69 \%$ & $-6.82 \%$ \\
\hline
\end{tabular}


As was done with the pressure drop results, a composite average difference from analytic was calculated for each model. These results are shown in Figure 8.6.

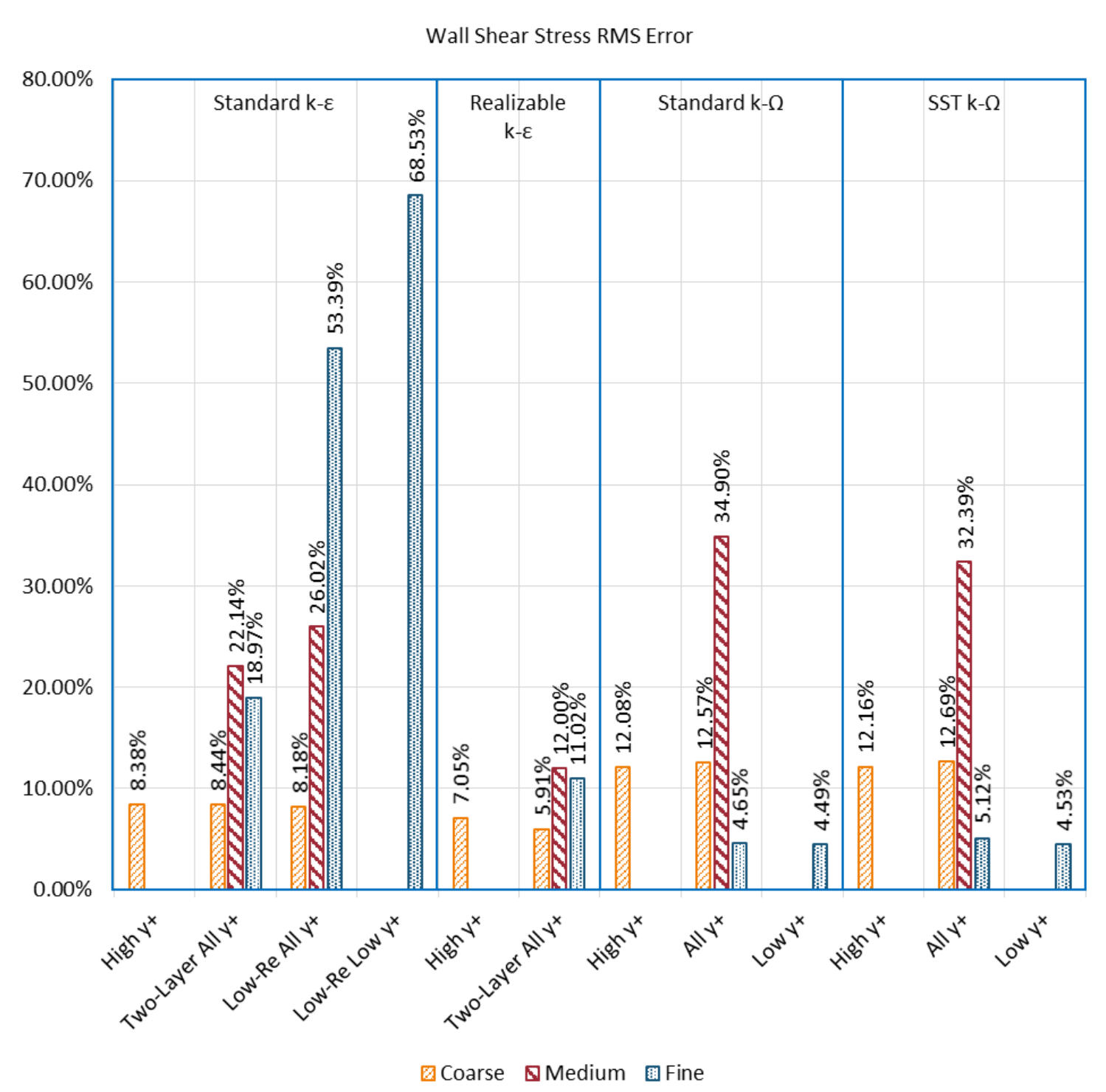

Figure 8.6. RMS wall shear stress error for all models

Again, both k- $\omega$ formulations with the fine mesh and All $\mathrm{y}+$ or Low $\mathrm{y}+$ wall treatment provided results closest to the analytic solution. Also, the k- $\varepsilon$ model again performed best with the coarse mesh and High y+ wall treatment. Table 8.13 through Table 8.16 
show the wall $y+$ values at the centroid of the near wall cell in the fully developed region. Within each table, the wall y+ for each inlet velocity, wall treatment, and mesh combination is provided. Note that the analytic value of the $y+$ solution varies depending on the mesh (i.e. wall distance) being utilized. Therefore, the analytic solutions corresponding to all three grids are provided. Additionally, the percent difference from the analytic solution (duplicated at the top of each table) is provided below the calculated Star-CCM+ wall y+ value. 
Table 8.13. Standard k- $\varepsilon$ wall $y+$ results

\begin{tabular}{|c|c|c|c|c|c|}
\hline $\begin{array}{c}\text { Wall } \\
\text { Treatment }\end{array}$ & Mesh & & $2 \mathrm{~m} / \mathrm{s}$ & $5 \mathrm{~m} / \mathrm{s}$ & $9 \mathrm{~m} / \mathrm{s}$ \\
\hline \multirow{3}{*}{ Analytic } & Coarse & & 31.8653 & 70.9811 & 119.3430 \\
\hline & Medium & & 7.9663 & 17.7453 & 29.8358 \\
\hline & Fine & & 0.2648 & 0.5900 & 0.9919 \\
\hline \multirow{2}{*}{ High y+ } & \multirow{2}{*}{ Coarse } & $y+$ & 33.8715 & 75.2191 & 126.2779 \\
\hline & & $\%$ Difference & $6.30 \%$ & $5.97 \%$ & $5.81 \%$ \\
\hline \multirow{6}{*}{$\begin{array}{c}\text { Two-Layer } \\
\text { All y+ }\end{array}$} & \multirow{2}{*}{ Coarse } & $y+$ & 34.0792 & 74.8852 & 125.9477 \\
\hline & & $\%$ Difference & $6.95 \%$ & $5.50 \%$ & $5.53 \%$ \\
\hline & \multirow{2}{*}{ Medium } & $y+$ & 9.2412 & 19.3103 & 31.2127 \\
\hline & & $\%$ Difference & $16.00 \%$ & $8.82 \%$ & $4.62 \%$ \\
\hline & \multirow{2}{*}{ Fine } & $y+$ & 0.2988 & 0.6109 & 1.0490 \\
\hline & & $\%$ Difference & $12.81 \%$ & $3.55 \%$ & $5.75 \%$ \\
\hline \multirow{6}{*}{$\begin{array}{c}\text { Low-Re } \\
\text { All y+ }\end{array}$} & \multirow{2}{*}{ Coarse } & $y+$ & 34.0845 & 74.8962 & 125.9355 \\
\hline & & $\%$ Difference & $6.96 \%$ & $5.52 \%$ & $5.52 \%$ \\
\hline & \multirow{2}{*}{ Medium } & $y+$ & 9.7717 & 19.4132 & 31.2762 \\
\hline & & $\%$ Difference & $22.66 \%$ & $9.40 \%$ & $4.83 \%$ \\
\hline & \multirow{2}{*}{ Fine } & $y+$ & 0.3243 & 0.7367 & 1.1694 \\
\hline & & $\%$ Difference & $22.44 \%$ & $24.88 \%$ & $17.90 \%$ \\
\hline \multirow{2}{*}{$\begin{array}{l}\text { Low-Re } \\
\text { Low y+ }\end{array}$} & \multirow{2}{*}{ Fine } & $y+$ & 0.3561 & 0.7291 & 1.2091 \\
\hline & & $\%$ Difference & $34.45 \%$ & $23.59 \%$ & $21.89 \%$ \\
\hline
\end{tabular}


Table 8.14. Realizable k- $\varepsilon$ wall y+ results

\begin{tabular}{|c|c|c|c|c|c|}
\hline $\begin{array}{c}\text { Wall } \\
\text { Treatment }\end{array}$ & Mesh & & $2 \mathrm{~m} / \mathrm{s}$ & $5 \mathrm{~m} / \mathrm{s}$ & $9 \mathrm{~m} / \mathrm{s}$ \\
\hline \multirow{3}{*}{ Analytic } & Coarse & & 31.8653 & 70.9811 & 119.3430 \\
\hline & Medium & & 7.9663 & 17.7453 & 29.8358 \\
\hline & Fine & & 0.2648 & 0.5900 & 0.9919 \\
\hline \multirow{2}{*}{ High y+ } & & $y+$ & 33.5843 & 74.6076 & 125.3201 \\
\hline & & $\%$ Difference & $5.39 \%$ & $5.11 \%$ & $5.01 \%$ \\
\hline \multirow{6}{*}{$\begin{array}{c}\text { Two-Layer } \\
\text { All y+ }\end{array}$} & & $y+$ & 33.5013 & 74.0934 & 124.8558 \\
\hline & & $\%$ Difference & $5.13 \%$ & $4.38 \%$ & $4.62 \%$ \\
\hline & & $y+$ & 8.5891 & 17.9647 & 30.0507 \\
\hline & 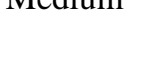 & $\%$ Difference & $7.82 \%$ & $1.24 \%$ & $0.72 \%$ \\
\hline & & $y+$ & 0.2844 & 0.6118 & 1.0048 \\
\hline & & $\%$ Difference & $7.37 \%$ & $3.71 \%$ & $1.30 \%$ \\
\hline
\end{tabular}


Table 8.15. Standard (Wilcox) k- $\omega$ wall $y+$ results

\begin{tabular}{|c|c|c|c|c|c|}
\hline $\begin{array}{c}\text { Wall } \\
\text { Treatment }\end{array}$ & Mesh & & $2 \mathrm{~m} / \mathrm{s}$ & $5 \mathrm{~m} / \mathrm{s}$ & $9 \mathrm{~m} / \mathrm{s}$ \\
\hline \multirow{3}{*}{ Analytic } & Coarse & & 31.8653 & 70.9811 & 119.3430 \\
\hline & Medium & & 7.9663 & 17.7453 & 29.8358 \\
\hline & Fine & & 0.2648 & 0.5900 & 0.9919 \\
\hline \multirow{2}{*}{ High y+ } & \multirow{2}{*}{ Coarse } & $y+$ & 34.7402 & 77.1452 & 129.4799 \\
\hline & & $\%$ Difference & $9.02 \%$ & $8.68 \%$ & $8.49 \%$ \\
\hline \multirow{6}{*}{ All $y+$} & \multirow{2}{*}{ Coarse } & $y+$ & 35.0838 & 76.9044 & 129.2059 \\
\hline & & $\%$ Difference & $10.10 \%$ & $8.34 \%$ & $8.26 \%$ \\
\hline & \multirow{2}{*}{ Medium } & $y+$ & 9.9732 & 19.8539 & 31.9654 \\
\hline & & $\%$ Difference & $25.19 \%$ & $11.88 \%$ & $7.14 \%$ \\
\hline & \multirow{2}{*}{ Fine } & $\mathrm{y}+$ & 0.2748 & 0.5887 & 0.9797 \\
\hline & & $\%$ Difference & $3.75 \%$ & $-0.21 \%$ & $-1.23 \%$ \\
\hline \multirow{2}{*}{ Low y+ } & \multirow{2}{*}{ Fine } & $y+$ & 0.2747 & 0.5902 & 0.9825 \\
\hline & & $\%$ Difference & $3.72 \%$ & $0.04 \%$ & $-0.95 \%$ \\
\hline
\end{tabular}


Table 8.16. SST (Menter) k-w wall y+ results

\begin{tabular}{|c|c|c|c|c|c|}
\hline $\begin{array}{c}\text { Wall } \\
\text { Treatment }\end{array}$ & Mesh & & $2 \mathrm{~m} / \mathrm{s}$ & $5 \mathrm{~m} / \mathrm{s}$ & $9 \mathrm{~m} / \mathrm{s}$ \\
\hline \multirow{3}{*}{ Analytic } & Coarse & & 31.8653 & 70.9811 & 119.3430 \\
\hline & Medium & & 7.9663 & 17.7453 & 29.8358 \\
\hline & Fine & & 0.2648 & 0.5900 & 0.9919 \\
\hline \multirow{2}{*}{ High y+ } & \multirow{2}{*}{ Coarse } & $y+$ & 34.5474 & 76.6633 & 128.6213 \\
\hline & & $\%$ Difference & $8.42 \%$ & $8.01 \%$ & $7.77 \%$ \\
\hline \multirow{6}{*}{ All $y+$} & \multirow{2}{*}{ Coarse } & $y+$ & 34.8977 & 76.4216 & 128.3459 \\
\hline & & $\%$ Difference & $9.52 \%$ & $7.66 \%$ & $7.54 \%$ \\
\hline & \multirow{2}{*}{ Medium } & $y+$ & 9.8333 & 19.5305 & 31.6220 \\
\hline & & $\%$ Difference & $23.44 \%$ & $10.06 \%$ & $5.99 \%$ \\
\hline & \multirow{2}{*}{ Fine } & $\mathrm{y}+$ & 0.2697 & 0.5838 & 0.9534 \\
\hline & & $\%$ Difference & $1.81 \%$ & $-1.05 \%$ & $-3.88 \%$ \\
\hline \multirow{2}{*}{ Low y+ } & \multirow{2}{*}{ Fine } & $y+$ & 0.2694 & 0.5845 & 0.9554 \\
\hline & & $\%$ Difference & $1.71 \%$ & $-0.93 \%$ & $-3.68 \%$ \\
\hline
\end{tabular}


Like the pressure drop and wall shear stress results, a RMS difference from analytic was calculated for each model. These results are shown in Figure 8.7.

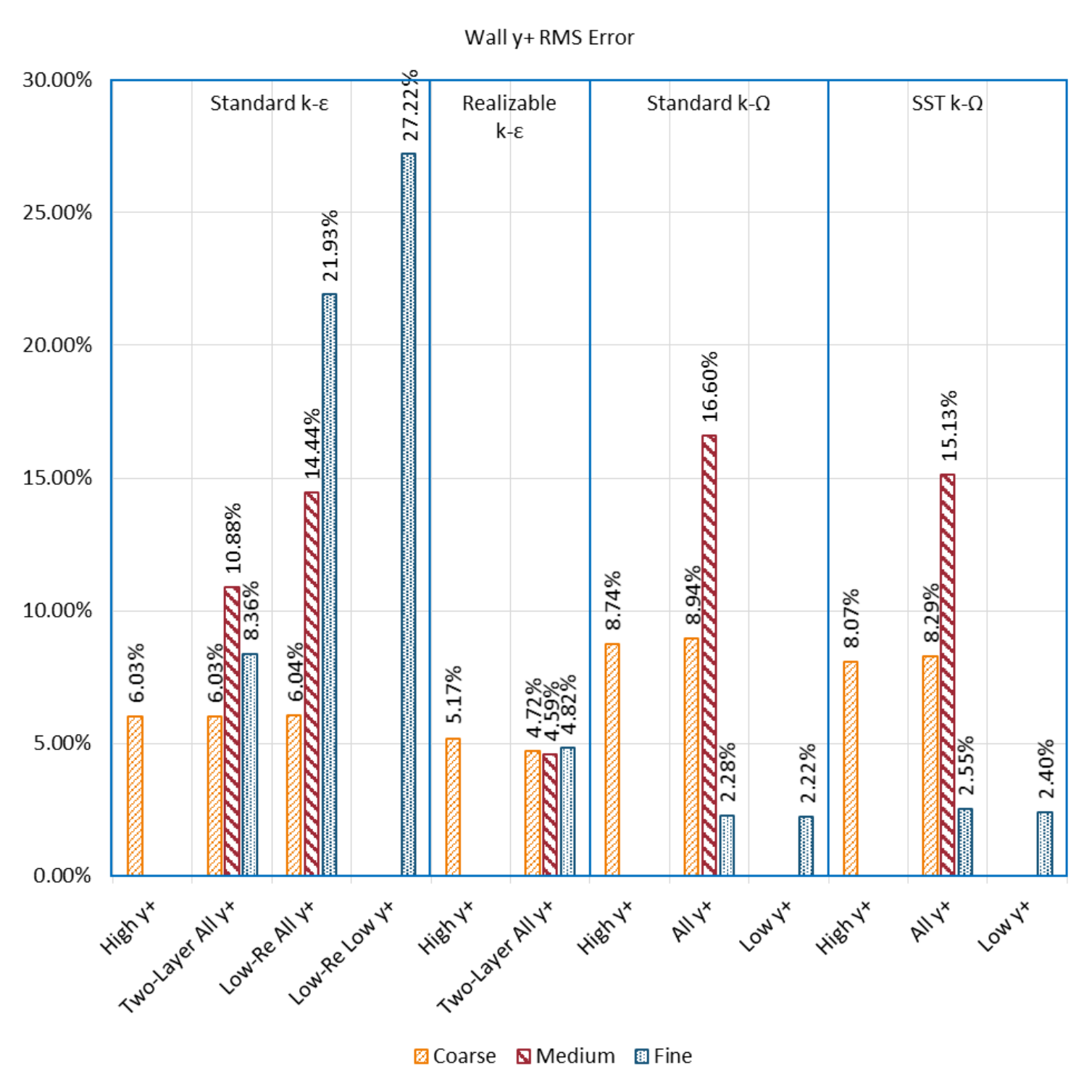

Figure 8.7. RMS wall y+ error for all models

Both the standard Wilcox and SST Menter k- $\omega$ models with fine meshes and All y+ or

Low $y+$ wall treatments matched closely to the expected analytic value in the fully developed region. 


\subsection{5: Summary}

For all three metrics (pressure drop, wall shear stress, and wall $y+$ ), the k- $\omega$ models with a fine wall mesh matched the analytic solution most closely. Additionally, using the All $y+$ or Low $y+$ wall treatment appears to have little effect on the solutions with this turbulence and mesh selection. Additionally, the k- $\varepsilon$ model performed reasonably well with the coarse mesh and High $\mathrm{y}+$ wall treatment. Low-Re $\mathrm{k}-\varepsilon$ formulations performed the worst. Also, the medium mesh performed poorly in nearly all scenarios.

It is clear from the results that the $\mathrm{k}-\omega$ turbulence model with a fine wall mesh is the best option for matching an analytic solution of flow through a wide, thin channel.

Additionally, there is negligible difference between the Standard Wilcox formulation and the SST Menter formulations. Finally, there was also negligible difference between the All $\mathrm{y}+$ and Low $\mathrm{y}+$ options. Therefore, CFD and FSI models should use the All y+ or Low $y+$ formulation with a fine near wall mesh.

Perhaps surprisingly, the k- $\varepsilon$ models performed best with the coarse mesh and high $\mathrm{y}+$ wall treatment. While the solutions with these models deviated from analytic more than the k- $\omega$ fine mesh models, they may still be useful in providing a quick solution in certain cases. From Table 8.3, the coarse mesh is approximately $1 / 4$ the density of the fine mesh, resulting in drastically shorter run times. This mesh and turbulence model combination could be used for scoping studies, where the need to run many simulations in a short amount of time outweighs the accuracy gains from the more computationally demanding $\mathrm{k}-\omega$, Low $\mathrm{y}+$ models. 
The medium mesh performed quite poorly in nearly all measures. This is not entirely surprising, given that the near wall centroid tended to be in the transition region - where the wall treatment functions are the least accurate.

Generally, k- $\omega$ turbulence models perform better near walls and k- $\varepsilon$ models perform better away from walls and in free-stream flows. This reality was the driving factor in creating the SST Menter k- $\omega$ model. Given the nearly two-dimensional, near wall flow through these channels, it is not surprising that the $\mathrm{k}-\omega$ models provided more reasonable results than k- $\varepsilon$ models on the same refined grid. Additionally, the historically good performance of $k-\varepsilon$ models away from walls correlates well with the results seen with the coarse mesh models.

Using the results of the turbulence model study and the methodology of section 2.3.3, the Grid Convergence Index (GCI) for the CFD static pressure was found to be $0.05 \%$. This is based on a factor of safety of 1.25. The full results from this study are shown in Table 8.17. 
Table 8.17. CFD grid convergence study results.

\begin{tabular}{|c|c|c|c|c|c|}
\hline & & & $\begin{array}{c}\varphi=\text { Pressure } \\
\text { Drop }\end{array}$ & $\begin{array}{c}\varphi=\text { Average } \\
\text { Plate Static } \\
\text { Pressure }\end{array}$ & $\begin{array}{c}\varphi=\text { Average } \\
\text { Plate Wall } \\
\text { Shear Stress }\end{array}$ \\
\hline \multirow{3}{*}{$\begin{array}{l}\text { Number of } \\
\text { Cells in CFD } \\
\text { Mesh }\end{array}$} & $\mathrm{N}_{1}$ & & & 8001361 & \\
\hline & $\mathrm{N}_{2}$ & & & 4326921 & \\
\hline & $\mathrm{N}_{3}$ & & & 2352096 & \\
\hline $\begin{array}{l}\text { Total CFD } \\
\text { Volume }\end{array}$ & $\forall$ & $\left(\mathrm{m}^{3}\right)$ & & $4.910 \times 10^{-4}$ & \\
\hline \multirow{3}{*}{$\begin{array}{l}\text { Representative } \\
\text { Cell Size }\end{array}$} & $\mathrm{h}_{1}$ & $(\mathrm{~m})$ & & $3.944 \times 10^{-4}$ & \\
\hline & $\mathrm{h}_{2}$ & $(\mathrm{~m})$ & & $4.841 \times 10^{-4}$ & \\
\hline & $\mathrm{h}_{3}$ & $(\mathrm{~m})$ & & $5.932 \times 10^{-4}$ & \\
\hline \multirow{3}{*}{ Solution } & $\varphi_{1}$ & $(\mathrm{~Pa})$ & 60539.71 & 22381.64 & 91.98 \\
\hline & $\varphi_{2}$ & $(\mathrm{~Pa})$ & 60010.24 & 22325.44 & 91.19 \\
\hline & $\varphi_{3}$ & $(\mathrm{~Pa})$ & 58788.44 & 21932.43 & 92.21 \\
\hline $\begin{array}{c}\text { Factor of } \\
\text { Safety } \\
\text { Grid }\end{array}$ & & & & 1.25 & \\
\hline $\begin{array}{l}\text { Convergence } \\
\text { Index }\end{array}$ & $\mathrm{GCI}_{\text {fine }^{21}}$ & & $0.82 \%$ & $0.05 \%$ & $3.55 \%$ \\
\hline
\end{tabular}

\section{3: Boundary Motion}

In fluid-structure interaction (FSI) models with moving plate boundaries, the CFD code has the added task of accommodating this boundary motion. As the boundaries move, the fluid mesh must adapt in some way to the new boundaries. In Star-CCM+, there are three potential ways to accomplish this:

1) Remeshing: after moving the boundaries, the previous mesh is deleted and a new mesh is created.

2) Morphing: the mesh nodes are moved to accommodate the new boundary location. 
3) Overset (Chimera): two meshes - background and overset - are laid on top of each other and the solver interpolates between them, with the overset mesh following the motion of the plate while the background remains fixed.

When considering remeshing, there are significant disadvantages in the time required. This technique was quickly found to be unfeasible, and the other two methods were considered in detail.

\subsection{1: Mesh Morphing}

The initial approach for adapting the fluid channels to the shape of the plate used the mesh morphing capability in Star-CCM+. This method reads the plate boundary motion into Star-CCM+ and interpolates that motion to the nodes along the surface. As the distance from the wall increases, the magnitude of node translation decreases, allowing the mesh to behave like a spring to accommodate the motion of the plate. Figure 8.8 shows a mesh that has morphed to accommodate the motion of a plate. The cells in the image are colored to represent the cell volumes. Darker blue cells are smaller, and green cells are larger. After the plate moves, the relative change in cell volumes becomes apparent. 


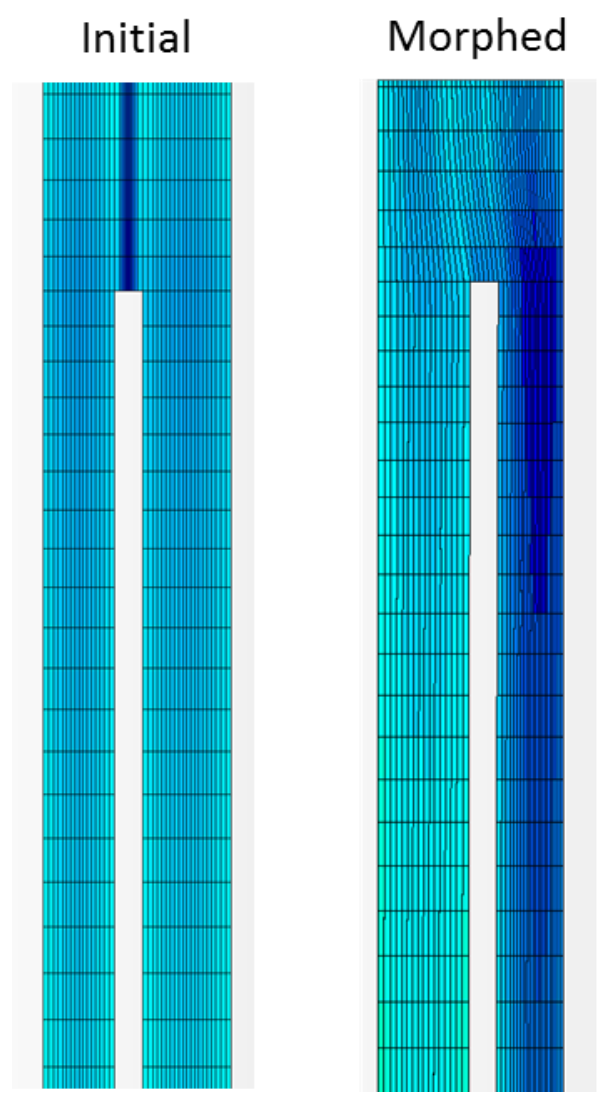

Figure 8.8. CFD mesh before and after morphing

While mesh morphing is fairly simple to set up and is computationally efficient, there are a number of issues which have to be addressed. First, as is shown in Figure 8.8, the cell thicknesses and volumes can change drastically as the plate moves. If the initial mesh was designed around specific sub-channel velocities or the specified wall treatment is dependent upon a range of wall $y+$ values, then dramatic changes in the cell shape may make the turbulence solver less accurate. Secondly, in cases where the plate move a large distance, there is potential for the cells to 'crush'. This occurs when the plate moves near the outer wall of the fluid model, and the cells in between become so small that they can invert. This results in a negative cell volume and leads the simulation to crash. 
Finally, in cases where the shrink and expand repeatedly, such as with an oscillating plate, the mesh quality can degrade [48]. This results from some hysteresis in the morphing algorithm that does not necessarily return a cell to its original shape if the motion is reversed. Figure 8.9 clearly shows this in a case with two plates that have oscillated over several morphings. This effect can largely be minimized if the mesh is always reset to its initial state prior to morphing, although this increases the computational time.

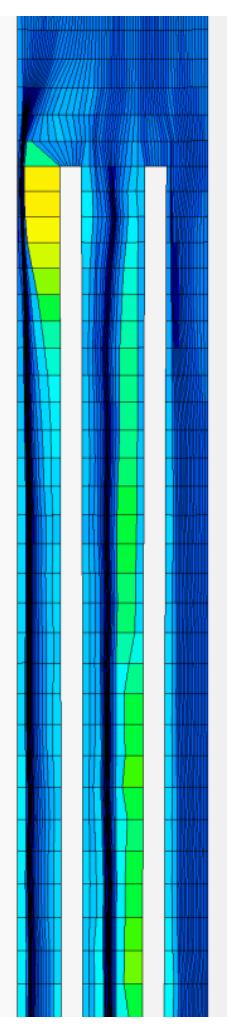

Figure 8.9. Low quality mesh resulting from repeated morphing

Given some of the difficulties with mesh morphing, it is a poor option for FSI simulations where the deflection is of the same order of magnitude as the geometry. In the cases presented here, the plate could easily deflect a millimeter or more through sub-channels 
that are about $2 \mathrm{~mm}$ thick. This could lead to the selected turbulence model or wall treatment becoming invalid, or to degradation of the mesh. Therefore, mesh morphing should only be used in cases where the plate motion is much smaller than the thickness of the fluid sub-channels.

\subsection{2: Overset Meshing}

Overset meshing utilizes two meshes - a background mesh and an overset mesh. The key attraction of this approach is that it allows the fluid cells to maintain their original shape throughout the simulation. As shown in Figure 8.10, the background mesh fills the entire fluid volume as though there were no plate present. The overset mesh is several cell layers thick and attached to the surface of the plate. These two meshes are then combined together and unused cells in the background mesh are deactivated. The fluid solution is interpolated between the two grids in the areas where the meshes overlap. 
1 - Background Mesh

- No cutout for plate

- Fixed (never morphs)

2 - Overset Mesh

- Mesh attached to cutout of plate

- Outer boundary 'floats' as plate deforms

3 - Resultant Mesh

- Background cells behind the plate and overset are ignored

- Interpolation between background and overset cells on floating outer boundary

Avoids cell thinning and degradation at FSI Interface

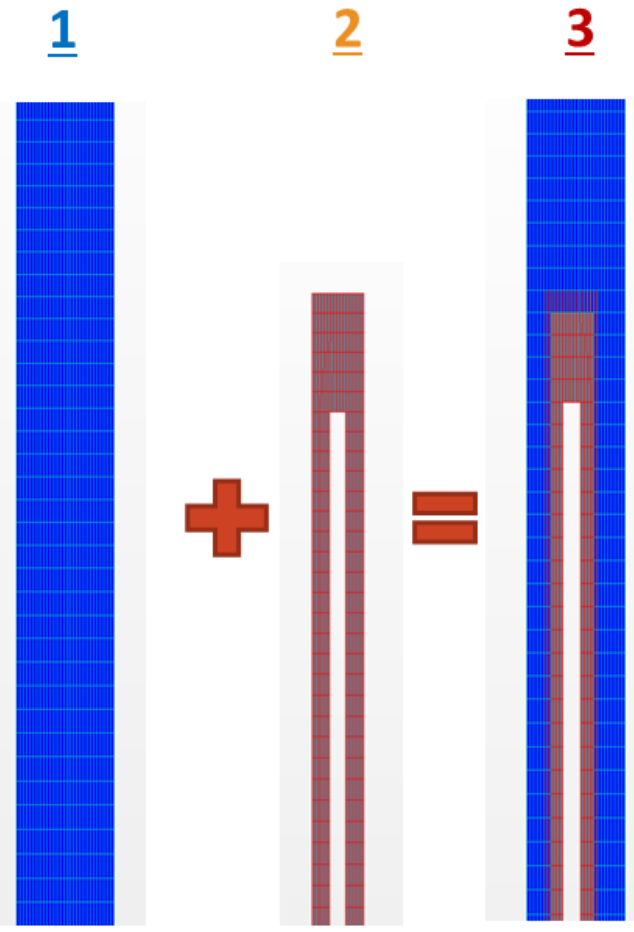

\section{Figure 8.10. Overset mesh setup}

One potential disadvantage of overset meshing is that in some cases, mass may not necessarily be conserved. If necessary, Star-CCM+ can overcome this limitation by adjusting the mass in the model through a source term. With the geometries under consideration here, this limitation is not a significant concern. Since the total fluid volume of the model is not changing and the fluid (water) is incompressible, mass removed from the deactivation of background cells will be added back in with the activation of other cells in the other fluid sub-channel. This has the effect of conserving mass throughout the entire model. Additionally, it provides some beneficial numeric stabilization by avoiding large swings in pressure from plate motion against an 
incompressible fluid. This results from the overset mesh allowing some mass transport between the channels as the plate moves.

\section{4: Final Fluid Model}

The final fluid mesh and turbulence model are based on the turbulence study, as well as investigation of the available mesh morphing options. Figure 8.11 provides a front view of the CFD model geometry, which is analogous to the experiment front view of Figure 4.4. 


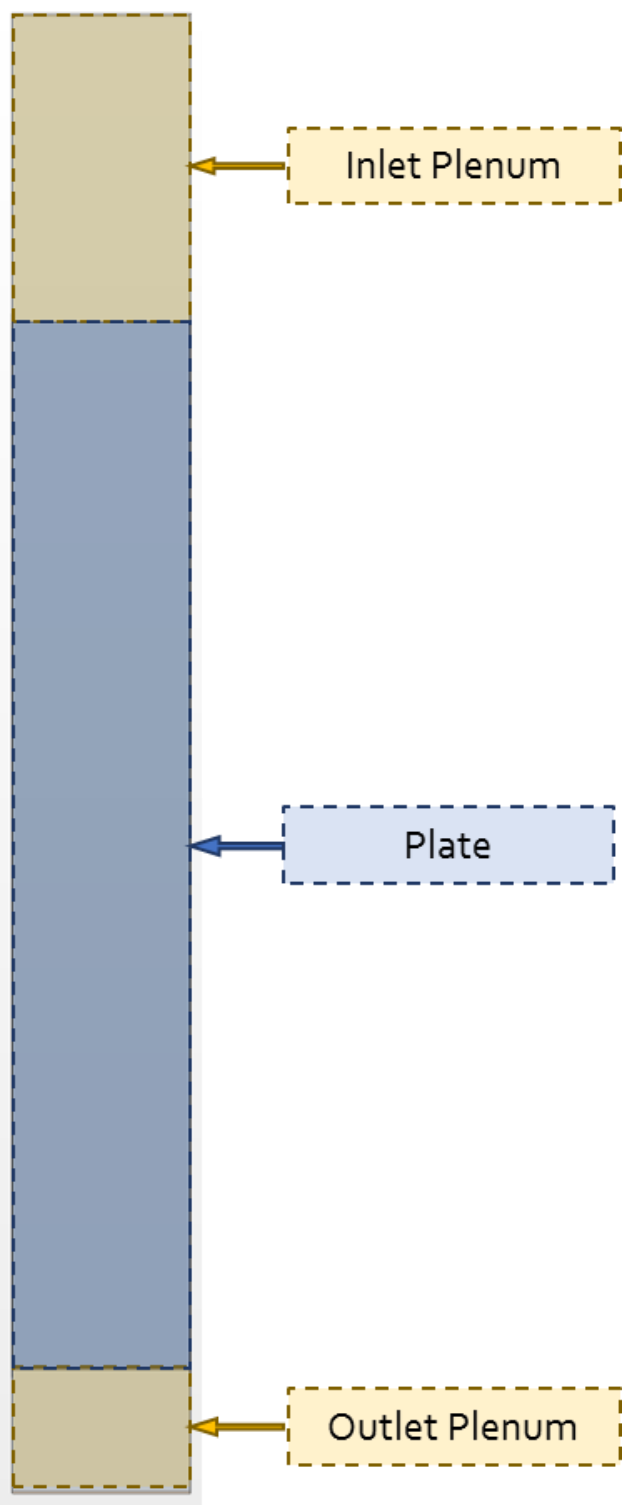

\section{Figure 8.11. CFD model front view}

The CFD (fluid) mesh is composed of two meshes. The background mesh (blue in Figure 10.2) is static and is generated without regard to the plate. The mesh is refined in the areas near the leading and trailing edges of the plate, where the flow splits and recombines. A cross-section of the fluid mesh taken near the plate leading edge is shown in Figure 8.12. Starting with the individual Background and Overset grids, an interpolated composite grid is created. The background grid is static, while the overset 
grid moves with the plate. Each time the overset grid moves, the final composite grid is re-calculated to activate $\&$ deactivate cells as needed.

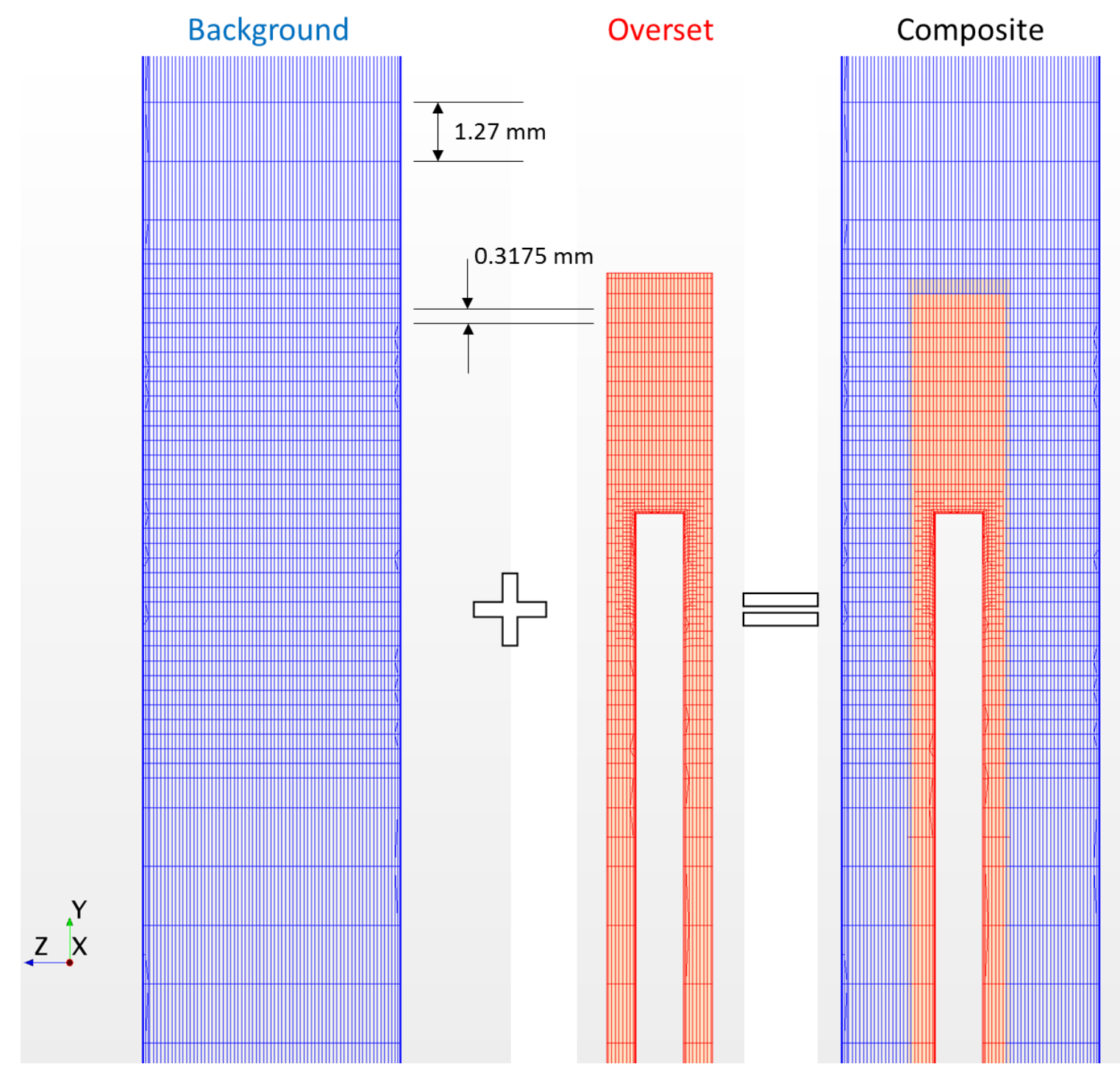

Figure 8.12. Overset mesh cross-section and interpolation

From Figure 8.12, the grid size in the flow direction of the refined region near the plate edge is shown. Additionally, the coarser grid size in the plenum and sub-channels is shown. Note that the same grid size was used in the $\mathrm{x}$ and $\mathrm{y}$ directions, while a much finer grid was used through the $\mathrm{z}$ direction. This allows the solver to continue capturing 
flow in the fluid channels, even as the plate deforms to near the outer wall of the fluid channels. A closer view of the near wall mesh for the background and overset meshes is shown in Figure 8.13.

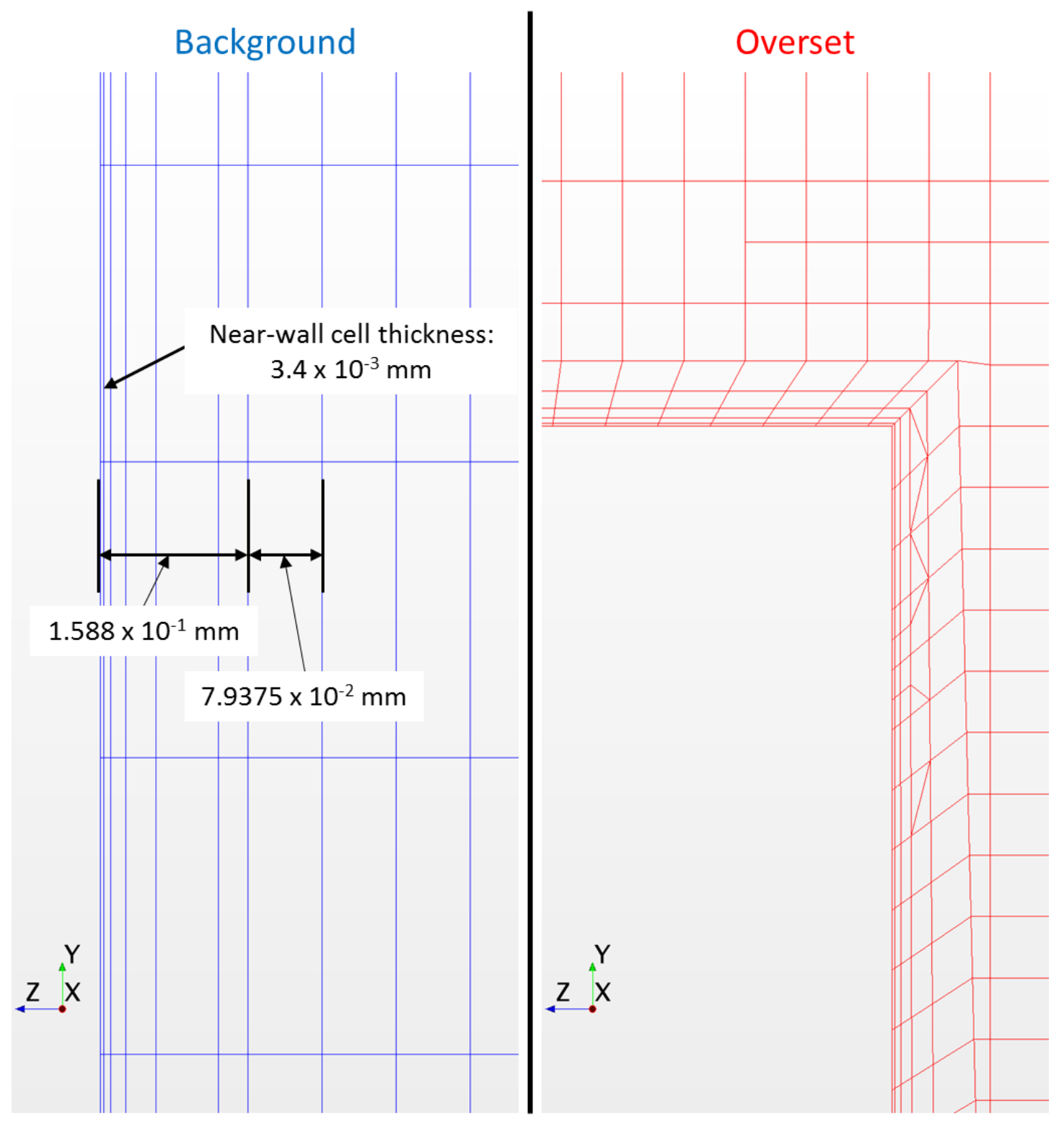

Figure 8.13. Near wall meshes

Based on the results of the turbulence study in section 8.2, the Standard (Wilcox) k- $\omega$ RANS turbulence model with a Low $y+$ wall treatment was selected. This is the best 
option for internal flow with a refined near wall mesh which can capture the boundary layer development. The near wall mesh was sized such that the $y+$ values of the first cell would be approximately equal to or less than one for the full range of flow rates being studies. 


\section{CHAPTER 9: FINITE ELEMENT ANALYSIS (FEA)}

\section{1: Plate Model}

The finite element model consists of a flat plate based on the actual plate used in the experiments. The wetted plate width (b) is $110.2868 \mathrm{~mm}$ (4.342 in.), length ( $\left.\mathrm{L}_{P}\right)$ is 647.7 $\mathrm{mm}$ (25.5 in.), and thickness (a) is $1.016 \mathrm{~mm}$ (40 mils). As shown in Figure 9.1, there is an additional $12.7 \mathrm{~mm}(0.5 \mathrm{in}$.$) along either side of the plate. This is the same width used$ for clamping the plate in the experiment, and these side surfaces are constrained from moving in the FEA model. 


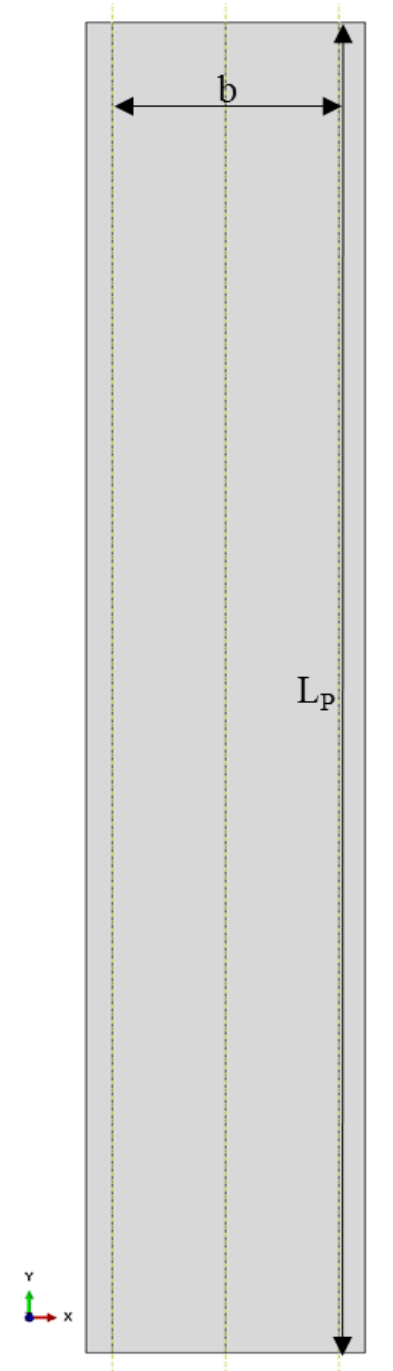

Figure 9.1. FEA model plate geometry

For an elastic structural analysis, several material properties need to be specified. The plate used in the experiments was 6061-T6 Aluminum and has the properties given in Table 9.1. Note that only elastic properties are considered, as the analysis assumes only elastic deformation. Plastic deformation is not expected as the stresses should remain below the yield point for the flow rates of interest. However, the stress field will be reviewed after each simulation to determine if the yield point was reached. 
Table 9.1. 6061-T6 FEA plate material properties

\begin{tabular}{ccc} 
Property & Symbol & Value \\
\hline Density & $\rho$ & $2700 \mathrm{~kg} / \mathrm{m}^{3}$ \\
Young's Modulus of Elasticity & E & $68.9 \mathrm{GPa}$ \\
Poisson's Ratio & $v$ & 0.33
\end{tabular}

\section{2: Element Type and Mesh}
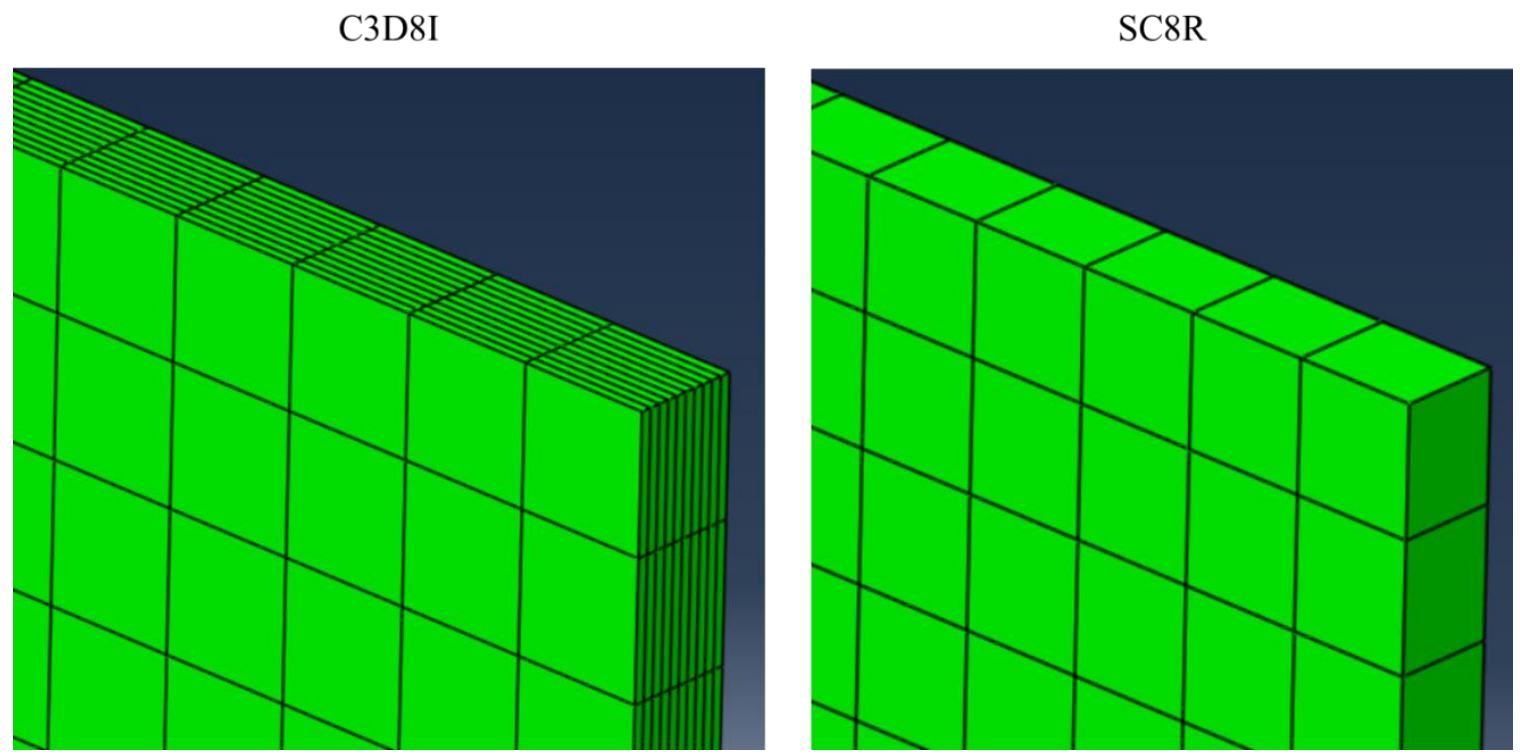

Figure 9.2. Close up of plate meshes. C3D8I on left, SC8R on right.

Two element types were considered for the plate model. The first model used 8 node, 3D solid continuum elements with incompatible modes (C3D8I). The element represents a true 3D volume, and therefore should include multiple elements through the thickness. These elements are capable of handling deflection and rotation in all directions, and are therefore more computationally expensive since more degrees of freedom need to be solved. 
The second model used 3D continuum shell elements (SC8R). Shell elements assume all deflection is along a single axis. This makes them particularly useful in plate type problems where essentially all deflection will be along a single axis - perpendicular to the plane of the plate. With a reduction in the degrees of freedom per element, the number of equations that need to be solved for each element drops dramatically. Additionally, shell elements are essentially two-dimensional. This means that they only need a single element through the plate thickness. This reduction in the number of elements ( $90 \%$ in this case) further reduces the number of equations that need to be solved for plate deflection. As simulation run time is directly tied to the number of equations in a model, this reduction yields huge savings in computational efficiency.

To determine if shell elements will work as well as 3D elements for the plate models of interest, a simple study was completed. A finite element representation of the plate was generated with both elements. The C3D8I mesh included 10 elements through the plate thickness, 106 through the width, and 510 along the length. This gives a total of 540600 elements. The SC8R mesh used the same number of elements along the width and length of the plate, but only one element through the thickness. Therefore, this mesh had 54060 elements. The two plate models were each loaded with five different uniform pressure loads and the centerline deflection was extracted from the results. The study was completed twice - once with non-linear geometry effects disabled, and a second time with them enabled. This will allow for comparison to the simple beam and Timoshenko plate models outlined in section 2.1.2. 
Figure 9.3 and Figure 9.4 show the axial deflection profiles for the plate of Figure 9.1 with dimensions of Table 4.2 under five different uniform distributed pressure loads with non-linear effects disabled. Disabling the non-linear effects calculation assumes that the membrane stresses are negligible. Therefore, the deflection solution is analogous to the simple beam calculation of equation 2.4. Figure 9.3 represents the C3D8I, solid continuum element mesh, while Figure 9.4 represents the SC8R, shell element mesh. The results between the two meshes differ by no more than $0.54 \%$.

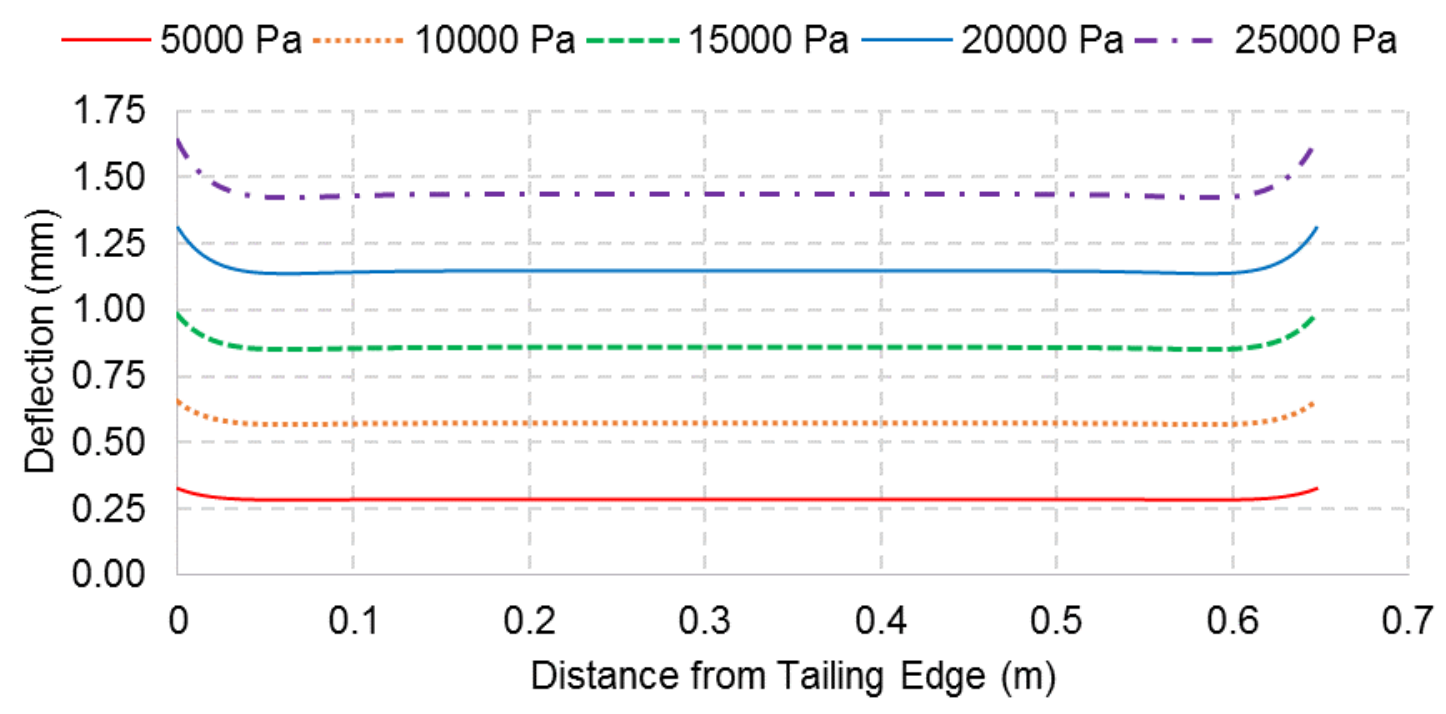

Figure 9.3. C3D8I, NLGeom off, axial deflection profiles under uniform pressure loads 


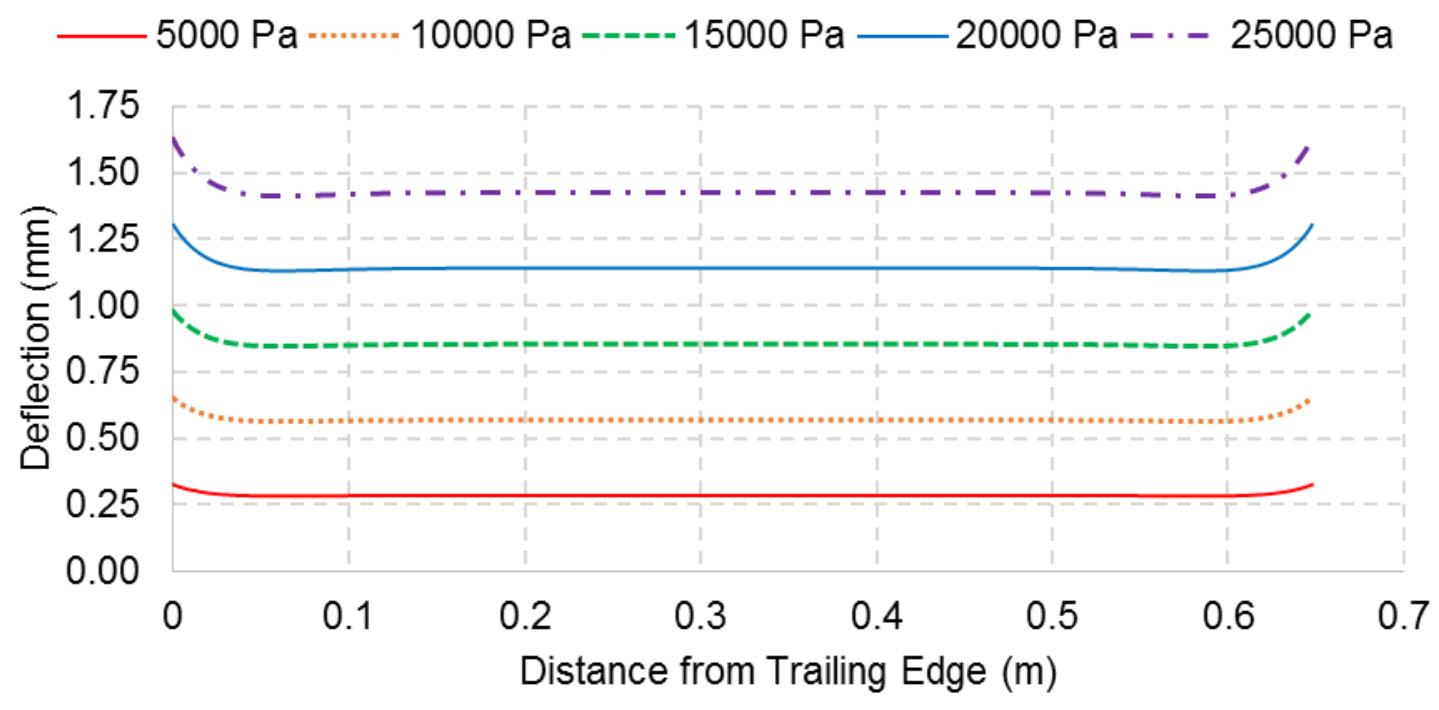

Figure 9.4. SC8R, NLGeom off, axial deflection profiles under uniform pressure loads

To determine if non-linear effects are significant in the plate solution, the simulations were repeated with non-linear geometry effects turned on. This solution accounts for membrane stresses, and is analogous to the Timoshenko plate deflection calculation of equation 2.6.

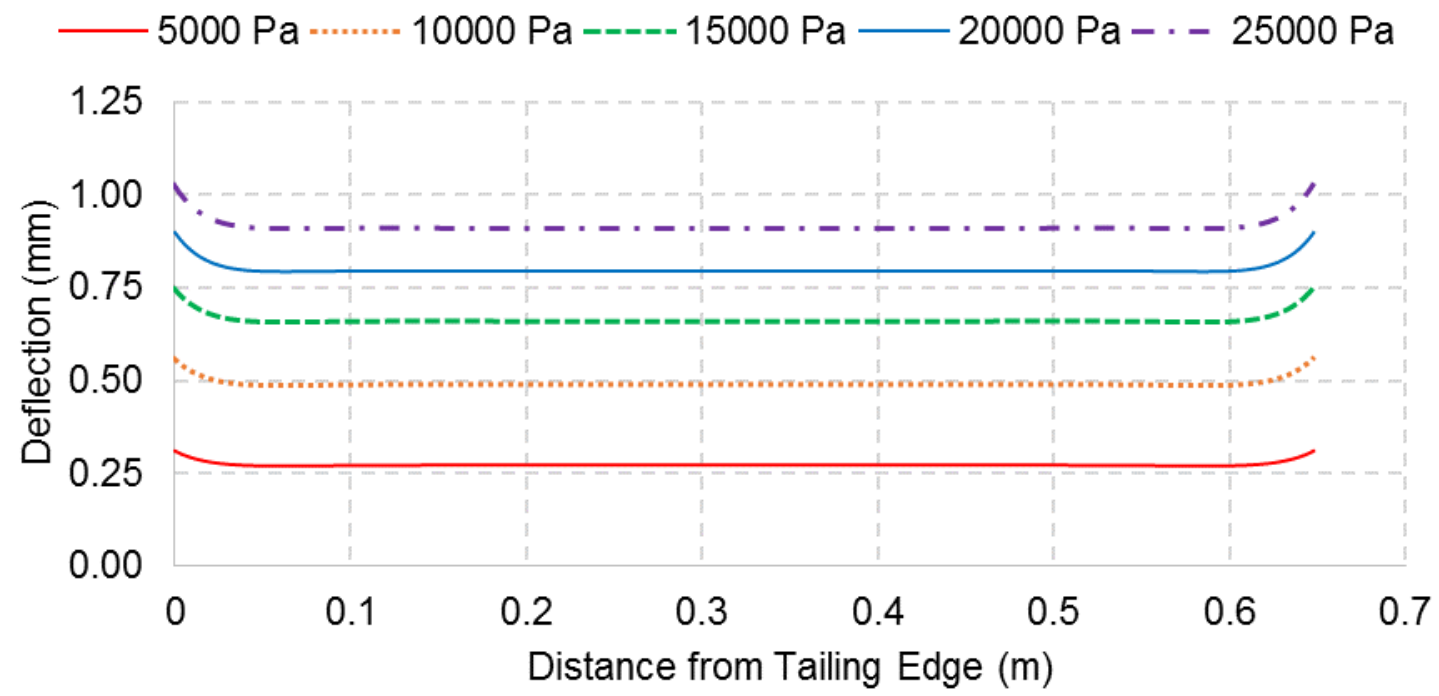

Figure 9.5. C3D8I, NLGeom on, axial deflection profiles under uniform pressure loads 


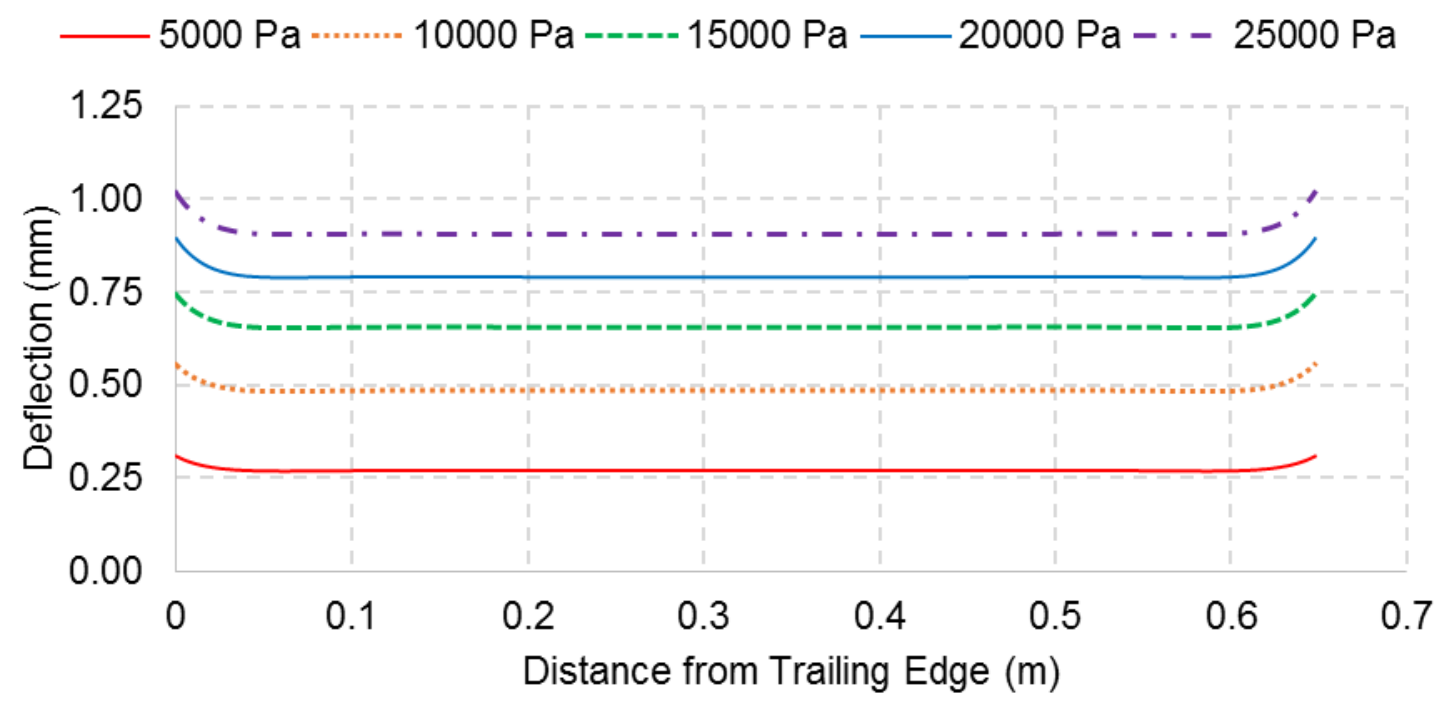

Figure 9.6. SC8R, NLGeom on, axial deflection profiles under uniform pressure loads

As before, the change in the mesh element had almost no impact on the results shown in Figure 9.5 and Figure 9.6. The largest difference between the two mesh solutions was $0.51 \%$. Additionally, the overall deflection magnitudes are greatly reduced with nonlinear effects included. This is not surprising, as the deflection magnitude is of the order of the plate thickness. Therefore, from these results, the plate models used in FSI calculations should include non-linear effects and the mesh should consist of shell elements. This combination provides the best combination of solution accuracy and computational efficiency.

\section{3: Solution Verification}

In order to determine if the deflections seen in the FEA models are the expected values, the numeric solutions presented in Figure 9.3 through Figure 9.6 can be compared against analytic models. From Section 2.1.2, a simple beam bending equation and a Timoshenko plate bending equation are each presented. Both of those equations were solved for the 
same plate thickness and width, and at the same pressures used in the FEA mesh study. The deflections at the plate midpoint of the FEA results were then extracted, and the analytic and numeric solutions were plotted against each other in Figure 9.7.

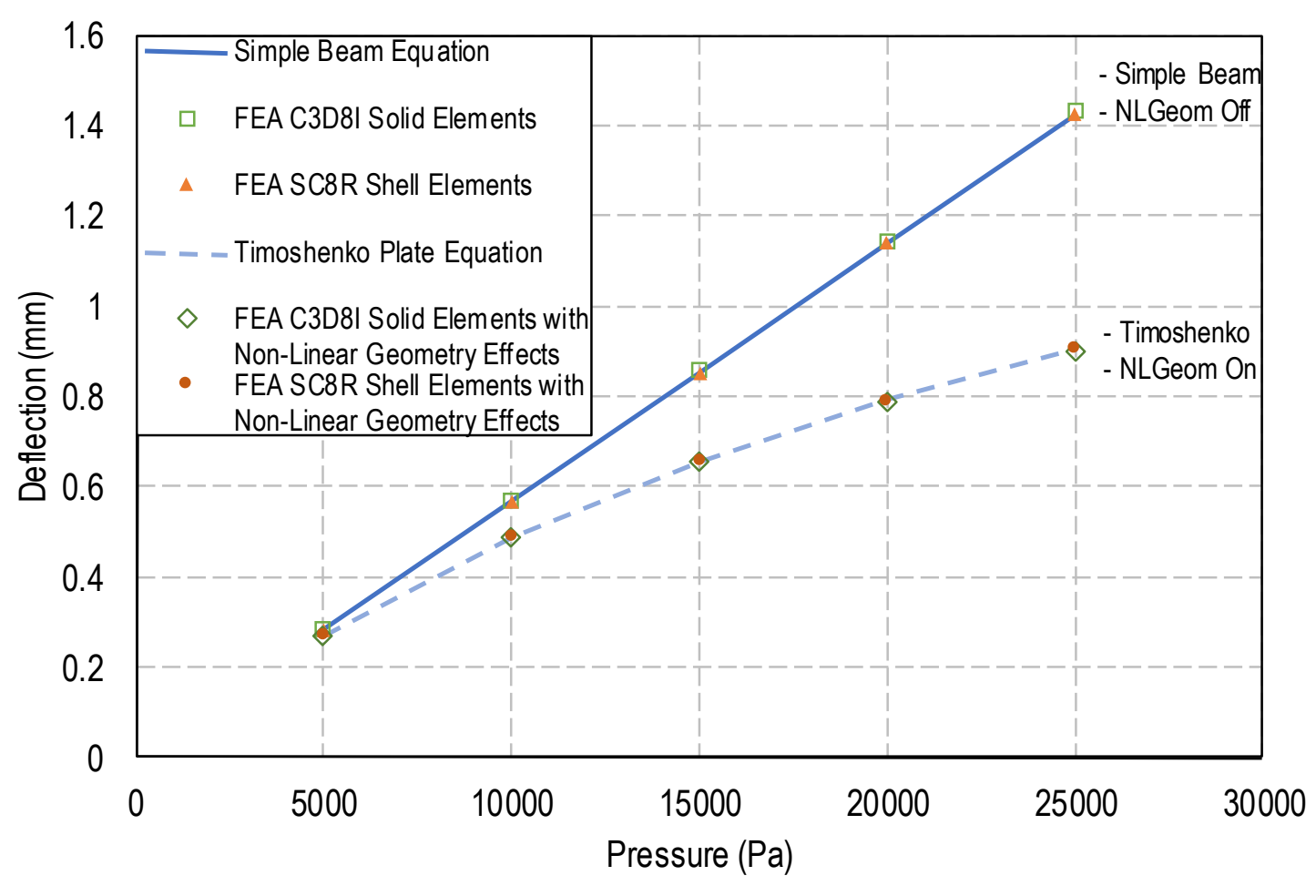

Figure 9.7. FEA comparison to analytic

From these results, the FEA models that did not consider non-linear effects almost perfectly track the simple beam analytic solution. The C3D8I solution differed from the analytic solution by $0.63 \%$, while the SC8R solution differed by only $0.07 \%$. For the models with non-linear effects included, the solutions nearly perfectly track the Timoshenko plate deflection model. The C3D8I NLGeom model differed from the analytic solution by $-0.02 \%$ to $0.04 \%$, while the SC8R NLGeom model differed by $0.43 \%$ to $0.58 \%$. 
Due to the extremely close matching to the analytic solution, this mesh and element type should work well in predicting plate behavior in FSI simulations. 


\section{CHAPTER 10: NUMERIC FLUID STRUCTURE INTERACTION (FSI)}

\section{1: Coupling CFD and FEA}

\subsection{1: Data Coupling Methods}

When work began on this project in 2009, Star-CCM+ and Abaqus had no simple means for communication between each other. Therefore, early investigations of FSI code coupling used a series of Fortan and java scripts to extract data from both codes at the shared boundaries for each time step. This data was written to a file, and then read into the other code so that the boundary conditions could be updated. The process was tedious and inefficient, with the act of writing data to the disk significantly slowing the data transfer process.

In the years since, $\mathrm{CD}$-Adapco and SIMULIA have integrated tools for data transfer into Star-CCM+ and Abaqus. Today, the user can setup an FSI simulation just as they would select a turbulence model or setup a mesh. These advancements have greatly increased the computational efficiency of FSI solutions, as well as allow for easier control of the solution process. The FSI Co-Simulation process is shown in Figure 10.1. The portion inside the dashed rectangle is completed only once in the Explicit coupling scheme, and multiple times in the Implicit coupling scheme. 


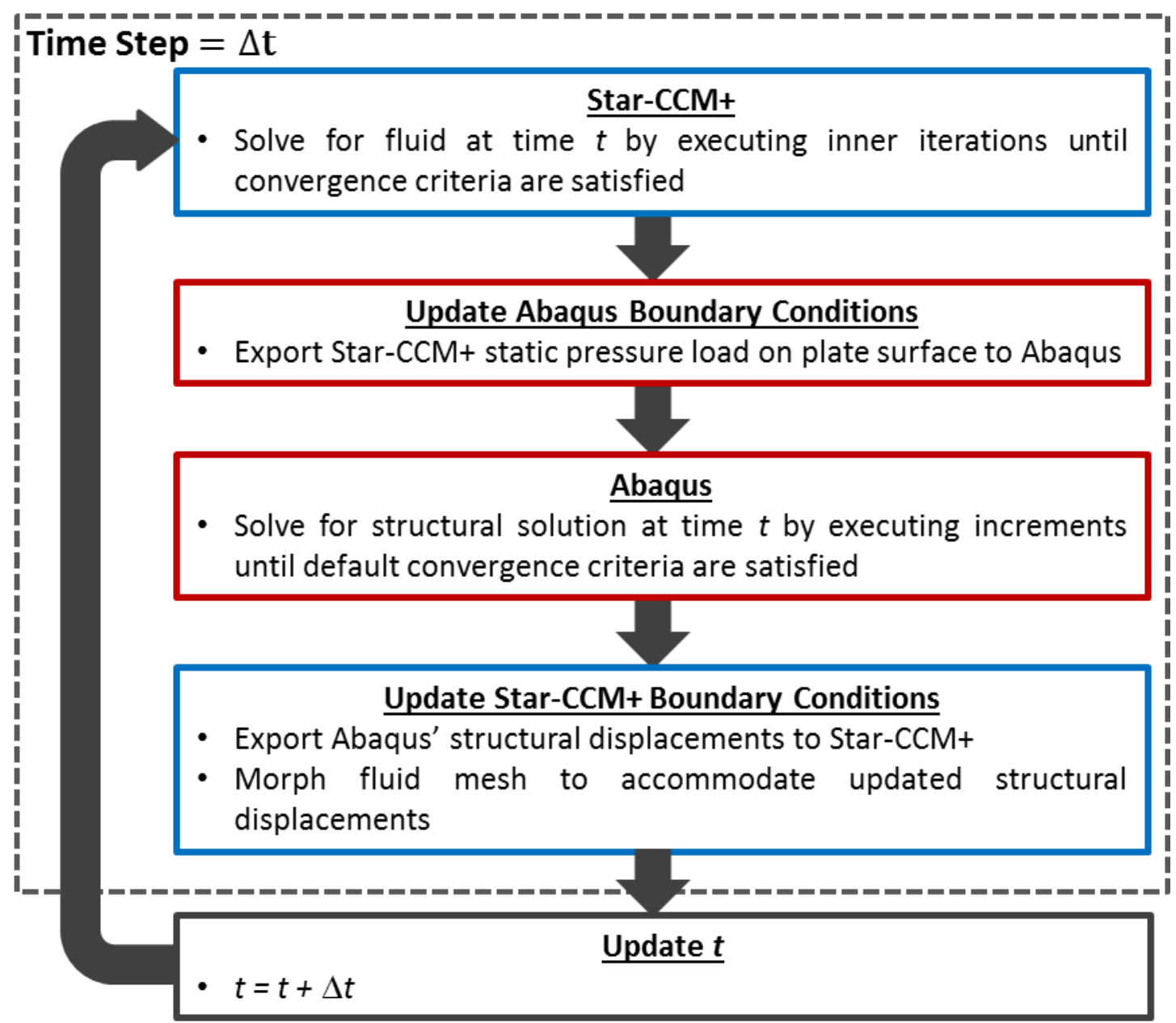

Figure 10.1. FSI Co-Simulation coupling process.

When setting up an FSI simulation, the pressure and wall shear stress solution is exported from the fluid model in Star-CCM+ and imposed on the plate surface in the Abaqus model. Abaqus then solves for the deflection of the plate with the prescribed pressure loading. Finally, the deflection of the nodes on the surface of the plate is exported from Abaqus to Star-CCM+ where the Overset mesh is morphed to accommodate the plate deflection. The interpolation between the Background and Overset cells is re-evaluated, and the fluid solution is advanced to the next coupling point. 
When setting up the FSI simulation, two coupling scheme options are available: Explicit and Implicit. Explicit coupling completes one exchange of data between the programs for each simulation time step. This method works well for simulations where time accuracy isn't important, or where the overall deflections are relatively small when compared to the mesh cell size. Implicit coupling would be better phrased as semiimplicit, as the two codes still work independently on their respective domains. The major difference in this scheme however is that the codes exchange data multiple times during a simulation time step. This allows the user to ensure that the simulation has converged to an accurate solution at each simulation time step before moving on to the next step. This technique has greater potential for time accurate solutions. Additionally, instabilities that may begin to develop in only a single time step in explicit coupling can be minimized as the simulation iterates longer at a given time step. The downside of this method is a dramatic increase in computational cost. A time step that takes only a single data exchange in explicit coupling, could take up to 50 data exchanges in implicit coupling. Since these simulations are largely concerned with the steady state, static deflection of a plate under flow, the explicit coupling scheme is the preferred option.

\subsection{2: Simulation Stabilization}

In addition to the coupling scheme, several other parameters play a significant role in how the solution evolves. These include the coupling time step, CFD Courant number specification, moving mesh grid flux control, and initial geometry morphing.

The time step size dictates how far the solution progresses before the programs update the plate boundary conditions. This step size solves the transient fluid and structural 
problems over the specified time, $\Delta \mathrm{t}$. A larger $\Delta \mathrm{t}$ allows the solution to progress further in each step, thereby reducing the number of data exchanges and increasing the speed of the overall simulation. However, if $\Delta \mathrm{t}$ is too large, the solution could begin to diverge as the plate may move too far with a given prescribed pressure field. If $\Delta t$ is too small, two potential problems may arise. The first being that the simulation will take an extraordinarily long time to complete. The second being that the solvers may begin to pick up higher frequency fluctuations in plate deflection, rather than the larger, bulk, steady-state deflections which are of primary interest. This can eventually lead to divergence and is commonly referred to as the added mass problem [49].

Inherent in the time step specification is the Courant-Friedrichs-Lewy condition (CFL or Courant number). The Courant number is a non-dimensional term which specifies how far a simulation advances in a given time step. For a three-dimensional case, the Courant number is defined by:

$$
C=\frac{u_{x} \Delta t}{\Delta x}+\frac{u_{y} \Delta t}{\Delta y}+\frac{u_{z} \Delta t}{\Delta z}
$$

Where:

$$
\begin{aligned}
& u_{\mathrm{i}}=\text { fluid velocity in the } i \text { direction }(\mathrm{m} / \mathrm{s}) \\
& \Delta t=\text { time step size }(\mathrm{s}) \\
& \Delta x, \Delta y, \Delta z=\text { grid size in the } \mathrm{x}, \mathrm{y}, \text { and } \mathrm{z} \text { directions }(\mathrm{m})
\end{aligned}
$$

For the geometry and coordinate system of interest here, it is reasonable to assume that all flow is in the $\mathrm{y}$ direction, and therefore the $\mathrm{x}$ and $\mathrm{z}$ terms of Eq. $10.1 \mathrm{can}$ be assumed as zero. Therefore the mesh size in the $y$ direction $(\Delta y)$, as well as the fluid velocity $\left(u_{\mathrm{y}}\right)$ are the driving factors in specifying the time step $(\Delta t)$ for a specified Courant number. 
Generally, the time step size for the simulations presented here was specified based on a Courant number between two and ten, with a lower Courant number at higher flow rates.

Within Star-CCM+, there is a secondary Courant number specification. This number is not specified through the time step size selection, but rather through a direct Courant number specification in the coupled-implicit fluid solver. Within a single time step in Star-CCM+, a given iteration of the solution is broken down into smaller sub-iterations. Star-CCM+ uses this directly specified Courant number to determine a pseudo-time step size for these sub-iterations. The Courant number specified to the coupled-implicit solver was typically set to five for the simulations presented here.

Finally, at higher flow rates there is a potential for the plate to oscillate somewhat between the fluid channels while the simulation drives towards convergence. When this happens, there is potential for the overset mesh surrounding the plate to degrade, as the very small cells at the plate surface are repeatedly deformed. On occasion, the cells will collapse and the simulation will crash due to a negative volume cell error. The problem is largely mitigated by instructing the mesh morpher in Star-CCM+ to always morph the mesh from the original $(t=0)$ position. This prevents small errors in the mesh morpher from building up through multiple morphings.

\section{2: Base (Ideal) Geometry Simulations}

Based on the experiment geometry of Figure 4.2 and Figure 4.3, numeric models of the fluid and plate were generated in Star-CCM+ and Abaqus, respectively. The mesh type, density, and turbulence models were based on the studies outlined in Chapter 8 and 
Chapter 9. The initial geometry considers the case of perfectly flat and uniform fluid channels. Figure 10.2 shows the combined plate and fluid models. The plate model is represented by the green mesh, while the fluid background and overset meshes are shown in blue and red. Note that half of the fluid mesh has been cut out of the image so that the plate is visible.

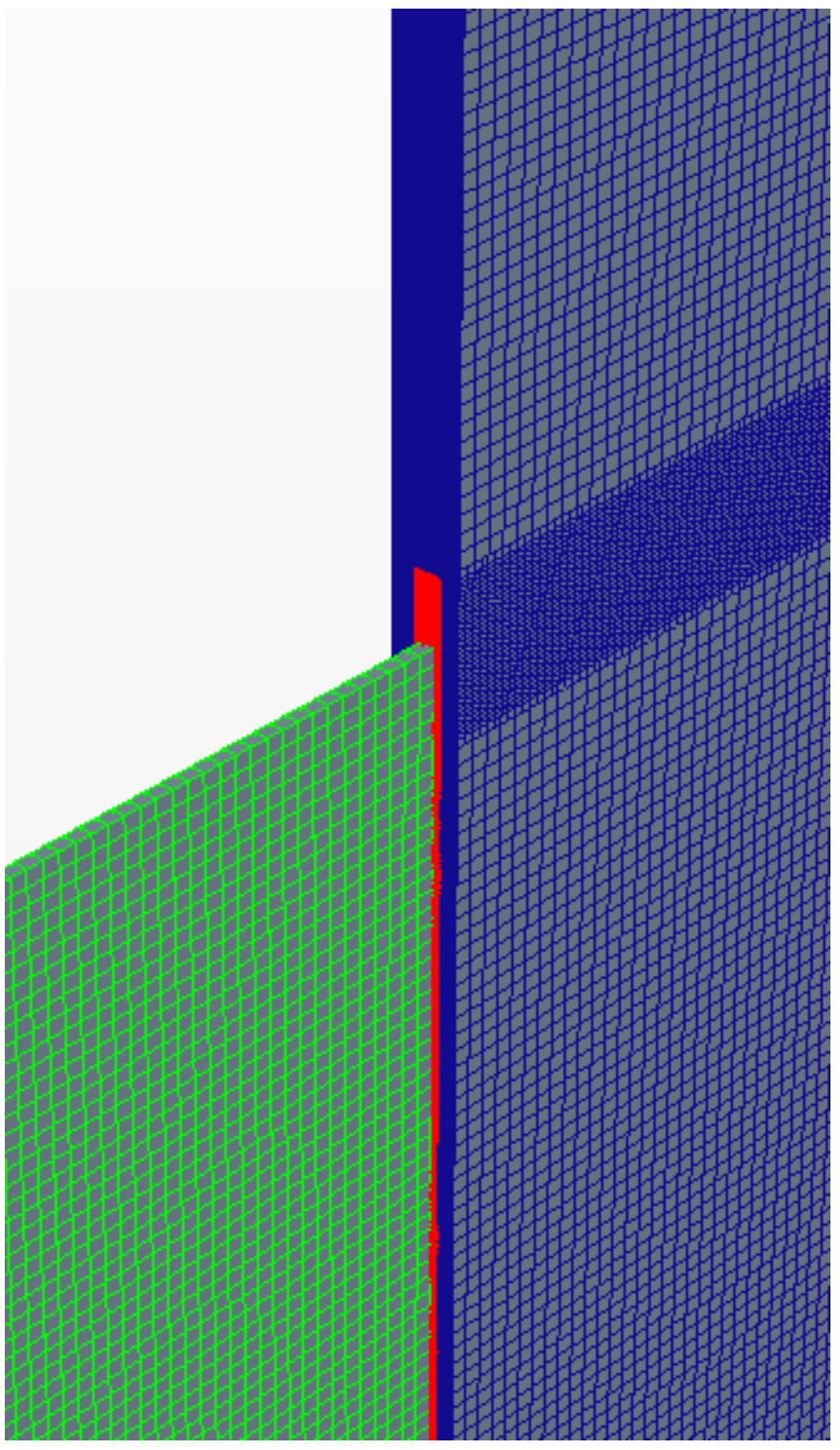

Figure 10.2. FSI geometry: Plate (green mesh); Background fluid (blue mesh); Overset fluid (red mesh) 


\subsection{1: FSI Model Setup}

The FEA shell element plate model outlined in Chapter 9 was coupled with the CFD fluid model outlined in section 8.4. Using this model, a range of simulations were set up to examine the behavior of a flat plate with free leading and trailing edges and clamped side edges. The flow rates and resulting average sub-channel velocities for the ideal geometry numeric simulations are based on the experimentally measured flow rates and are shown in Table 10.1.

Table 10.1. Ideal geometry FSI parameters

\begin{tabular}{ccccc}
\hline $\begin{array}{c}\text { Flow Rate } \\
(\mathbf{k g} / \mathbf{s})\end{array}$ & $\begin{array}{c}\text { Average Fluid } \\
\text { Channel Velocity } \\
(\mathbf{m} / \mathbf{s})\end{array}$ & $\begin{array}{c}\text { Coupling } \\
\text { Scheme }\end{array}$ & $\begin{array}{c}\text { Step Size } \\
(\mathbf{s})\end{array}$ & CFL \\
\hline 1.04 & 2.07 & Explicit & $6.35 \mathrm{E}-3$ & 10.34 \\
1.74 & 3.46 & Explicit & $1.59 \mathrm{E}-3$ & 4.33 \\
2.30 & 4.57 & Explicit & $1.27 \mathrm{E}-3$ & 4.57 \\
2.60 & 5.17 & Explicit & $1.20 \mathrm{E}-3$ & 4.88 \\
2.86 & 5.69 & Explicit & $9.27 \mathrm{E}-4$ & 4.15 \\
3.43 & 6.82 & Explicit & $6.62 \mathrm{E}-4$ & 3.55 \\
4.34 & 8.63 & Implicit & $4.28 \mathrm{E}-4$ & 2.91 \\
\hline
\end{tabular}

From Table 10.1, it should be noted that the more efficient explicit coupling scheme worked well for all but the highest flow rate. At higher flow rates, where larger plate deflection is noted, the simulation has a tendency to be more unstable. Using more data exchanges within a time step allows for a more gradual updating of the fluid pressure and plate deflection solutions, thereby greatly increasing simulation stability. 
The time stepping scheme was based on the average channel velocity and the length of the cells in the flow direction in the sub-channels. Stable time step sizes, as shown in Table 10.1, generally resulted in Courant numbers between approximately two and ten. As the fluid velocity increased and plate deflection increased, it was necessary to reduce the step size in order to maintain simulation stability.

\subsection{2: Ideal Geometry FSI Results}

FSI simulations were completed for the flow rates outlined in Table 10.1. Since deflection is the primary concern, the plate deflection profile was extracted from the Abaqus simulation along the axial centerline. An example of this process from the 3.43 $\mathrm{kg} / \mathrm{s}$ simulation is shown in Figure 10.3. A path is created on the surface of the plate from the trailing edge to the leading edge. The deflection at every node along that path is extracted and plotted to give the axial deflection profile. The axial deflection profiles for the simulations outlined in Table 10.1 are shown in Figure 10.4. 


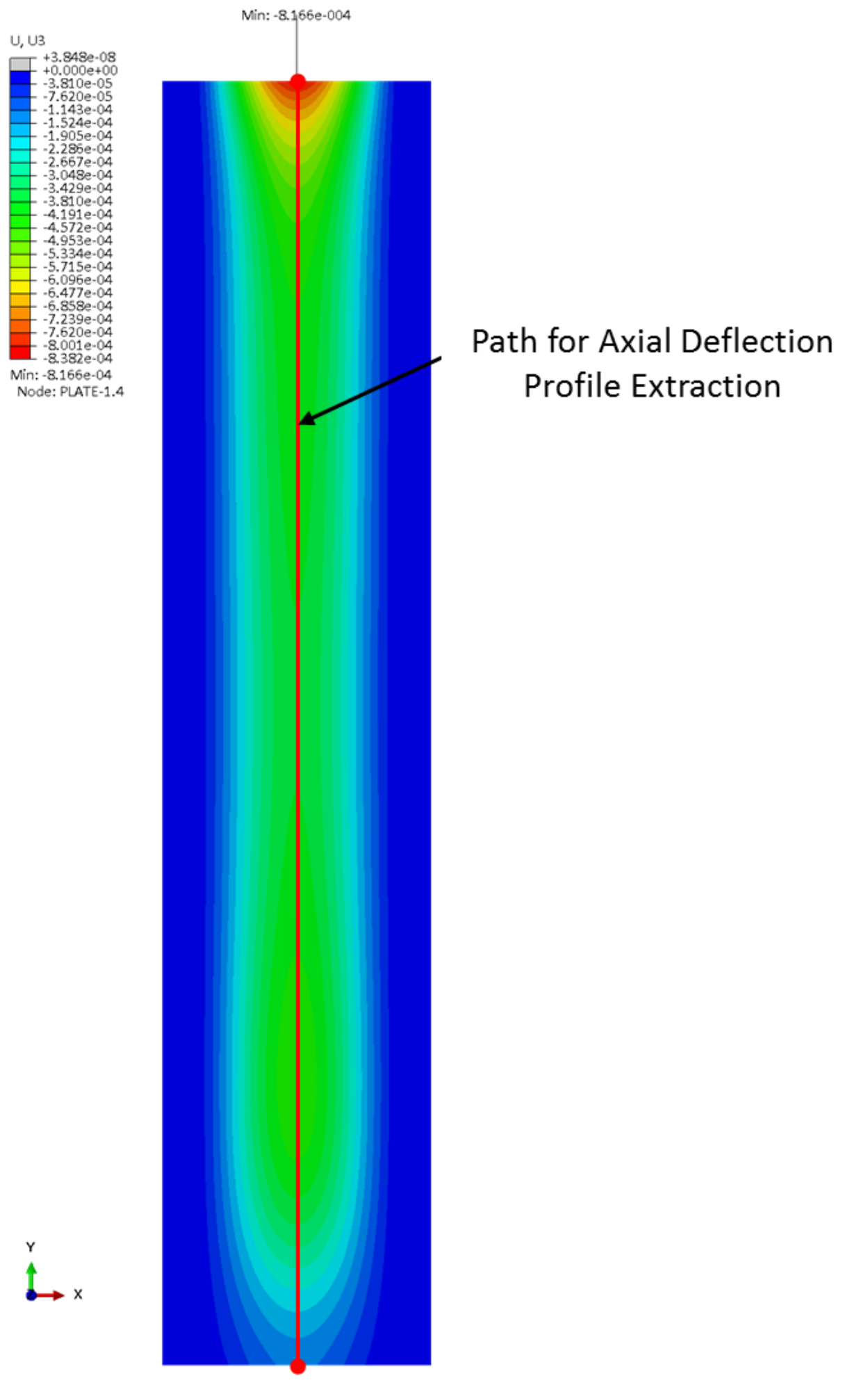

Figure 10.3. $3.43 \mathrm{~kg} / \mathrm{s}$ Abaqus deflection profile 


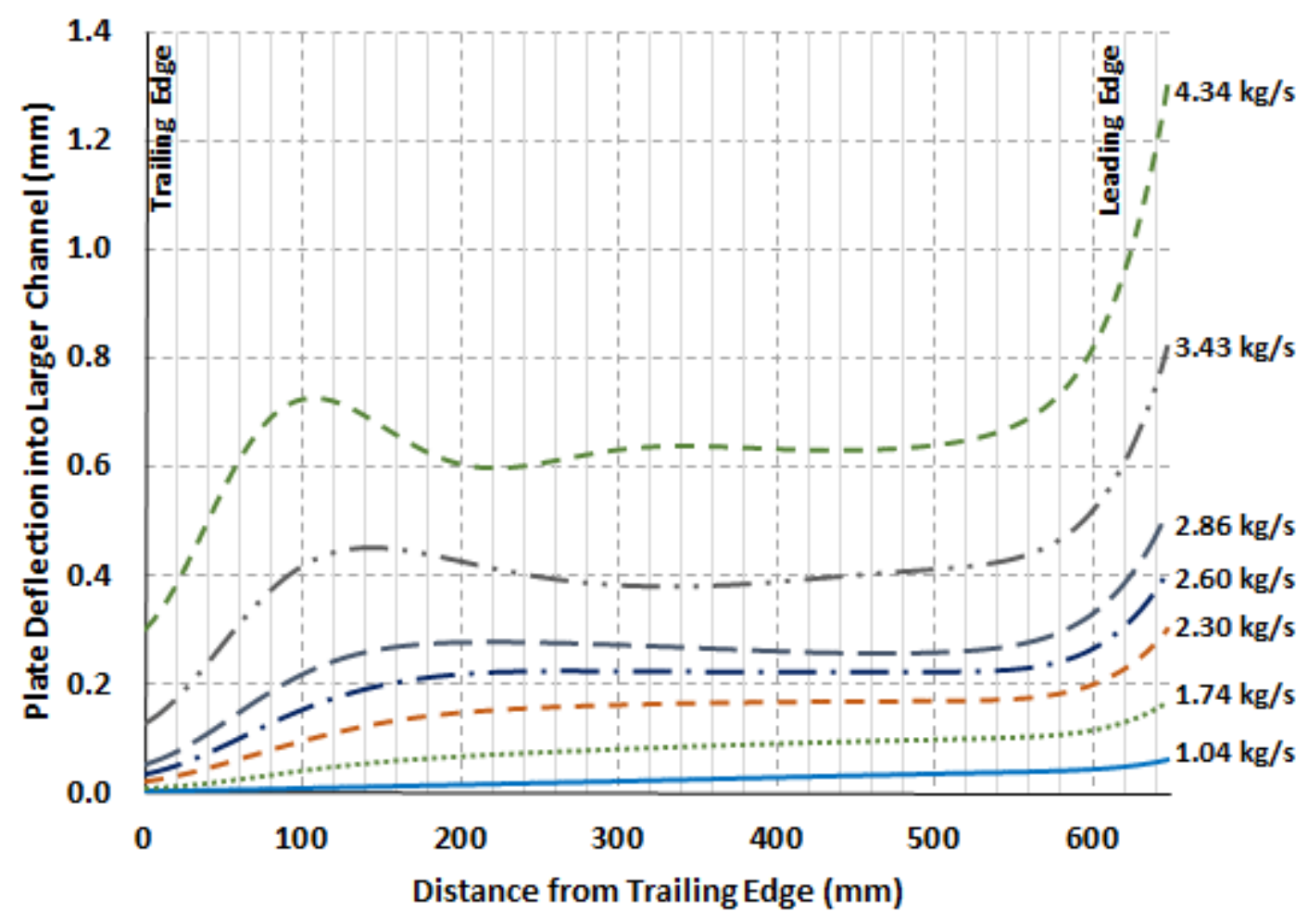

Figure 10.4. Axial deflection profiles for Ideal geometry FSI simulations

Much like the deflection profiles which are extracted from Abaqus, pressure profiles can be extracted from Star-CCM+. This is accomplished by creating line probes through each fluid channel and taking the difference between the pressure in the smaller channel and the larger channel. Assuming the smaller channel has a higher pressure than the larger channel, this would represent the pressure that tends to push the plate into the larger channel. For the $3.43 \mathrm{~kg} / \mathrm{s}$ case, this pressure differential extraction process is illustrated in Figure 10.5. 


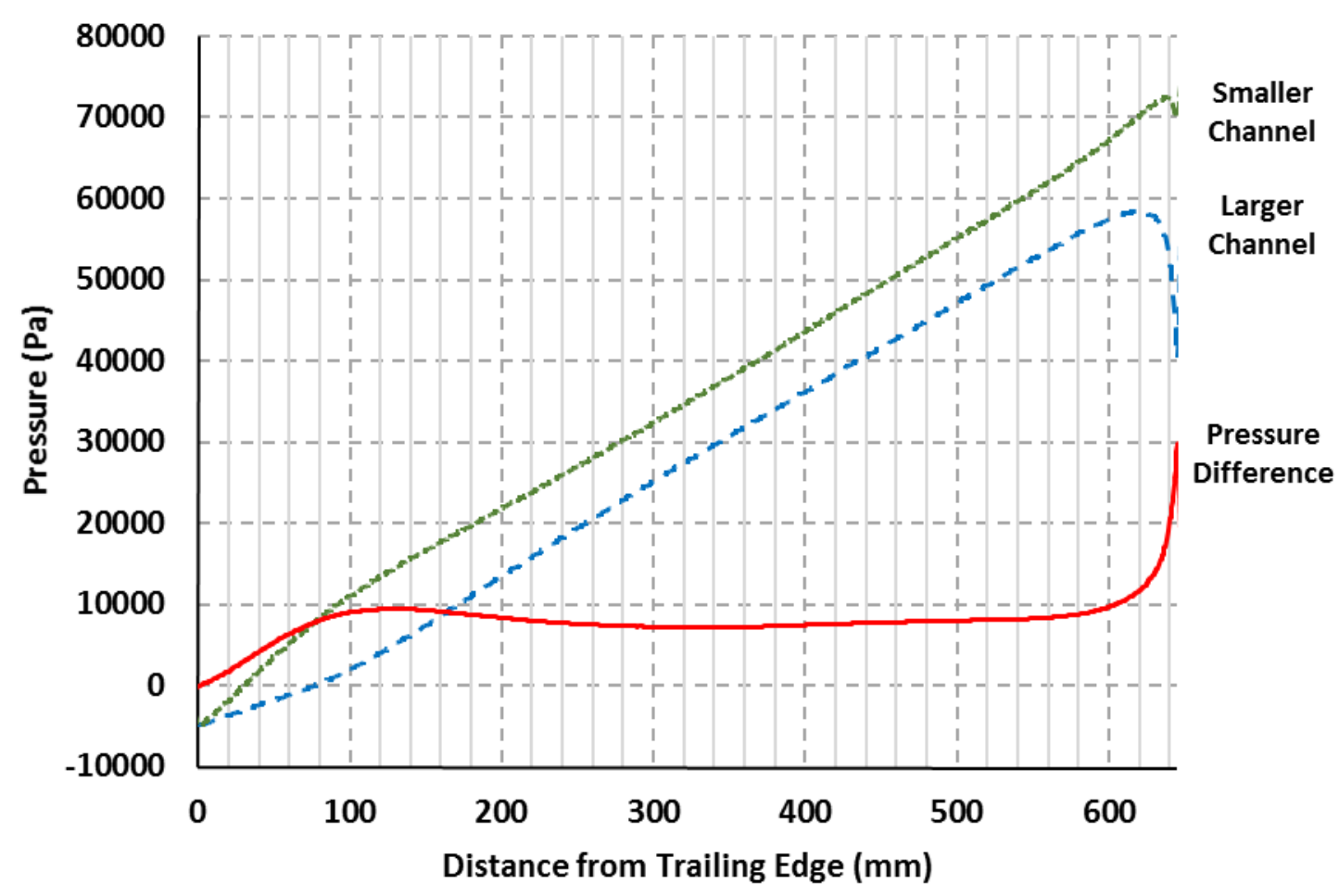

Figure 10.5. Channel pressure data at $3.43 \mathrm{~kg} / \mathrm{s}$ flow

This pressure data extraction process was completed for all flow rates shown in Table 10.1, and the resulting pressure differentials (solid red line in Figure 10.5) are shown in Figure 10.6. Note the steep gradient in the pressure profile near the leading edge of the plate. This results for the sudden flow contraction as the fluid enters the sub-channels from the inlet plenum. 


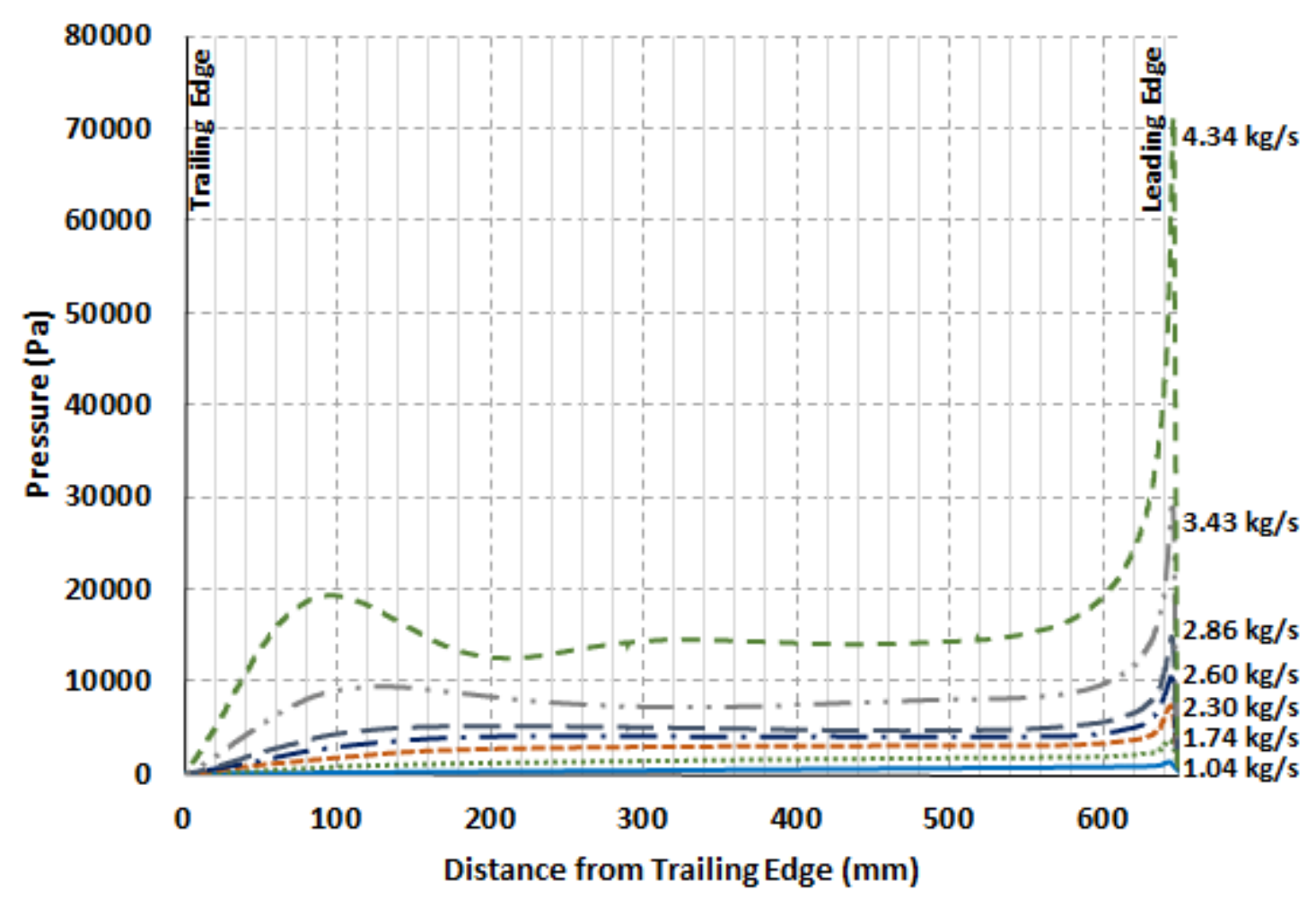

Figure 10.6. Pressure force acting on plate for Ideal geometry simulations

As expected, as the flow rate increases, the resulting pressure force on the plate and deflection also increases. Additionally, as the flow rate increases, a region of increased pressure and deflection begins to appear near the trailing edge of the plate. This is seen in both the deflection profiles of Figure 10.4 and the pressure profiles of Figure 10.6. Most importantly, when compared to the experiment results of section 5.3, the deflection profiles differ significantly in both their qualitative shape and quantitative magnitude. This has led to the need for more detailed numeric models. 


\section{CHAPTER 11: AS-BUILT NUMERIC FSI SIMULATIONS}

\section{1: Theory}

As the ideal geometry FSI models have shown significant deviation from measured experiment results, it is proposed that a more thorough characterization of the experiment geometry might bring the numeric models closer to reality. Using the high density channel mapping data of Figure 5.4, the outer walls of the fluid background mesh of Figure 10.2 are to be modified to capture the variations of the experiment geometry. This modification transforms the Ideal fluid model discussed previously into an As-Built fluid model, which more closely matches the experiment reality. By removing the assumption of perfectly flat and uniform channels from the CFD model, the fluid flow solution will account for variations in the pressure field affecting the plate deflection.

\section{2: CFD Background Mesh Modification}

Since the experiment mapping grid has a different density than the CFD grid, interpolation is necessary in order to approximate the channel thickness at all points on the CFD outer walls. To do this, a python script was created to read in the channel measurement data and complete a linear interpolation onto a grid of similar density to the CFD grid. This script is provided in Appendix C.

When it comes to actually morphing the CFD mesh, there are a couple of options, depending on how the mesh was generated. If the mesh was created in Abaqus for export to Star-CCM+, the it is relatively simple to access the mesh nodal locations in the Abauqs 
inp file with a python script. From there, the $\mathrm{z}$ component of the nodal locations can be 'scaled' in order to move the nodes on the outer wall to locations which match the channel mapping. For example, at a given $\mathrm{x}, \mathrm{y}$ location, the ideal geometry specifies a $\mathrm{z}$ nodal location on the outer channel wall which gives a channel thickness (i.e. distance from the plate) of $h_{\text {ideal }}$. If the experiment was measured to be a different thickness, $h_{\text {expt }}$, then the node on the outer wall would need to move along the $\mathrm{z}$ axis by some distance $\Delta \mathrm{h}$ $=\mathrm{h}_{\text {expt }}-\mathrm{h}_{\text {ideal }}$. Since $\Delta \mathrm{h}$ tends to be small, all the other nodes along the $\mathrm{z}$ axis at that $\mathrm{x}, \mathrm{y}$ location can be slightly shifted, based on how near they are to the outer wall. Nodes on the outer wall are moved the full distance $\Delta \mathrm{h}$, whereas nodes on the plate surface are not moved at all. Nodes halfway between the outer wall and the plate move a distance $\Delta \mathrm{h} / 2$.

An alternative background mesh morphing option is used if the mesh is generated within Star-CCM+. For the simulations presented here, this is the method that was utilized. Since Star-CCM+ does not allow the user access to the nodal coordinates, the user is forced to rely on the built in mesh morphing algorithm. This requires importing a table of initial $\mathrm{x}, \mathrm{y}, \mathrm{z}$ locations on the ideal geometry outer wall surface and the desired grid translation at each of those locations $(\Delta \mathrm{x}, \Delta \mathrm{y}, \Delta \mathrm{z})$. For the simulations presented here, the initial $\mathrm{x}, \mathrm{y}$ coordinates are derived from the channel mapping, and the initial $\mathrm{z}$ coordinate is based on the outer wall location in the ideal CFD model. Since translation is required only in the $\mathrm{z}$ direction, $\Delta \mathrm{x}=\Delta \mathrm{y}=0$, and $\Delta \mathrm{z}=\Delta \mathrm{h}$, as prescribed in the previous paragraph. Star-CCM+ uses this table of location and translation values to interpolate the outer wall surface to match the experiment channel measurements. 
It should be noted that geometry adjustment process must be started with the Ideal geometry fully meshed. If the user attempts to mesh the geometry after morphing the outer walls, the Star-CCM+ meshing algorithm will force a significantly higher density mesh onto the model. The Ideal geometry CFD mesh of section 8.4 relied on scaling the cell sizes up in the $\mathrm{x}$ and $\mathrm{y}$ (width and length) directions, and scaling them down in the $\mathrm{z}$ (channel thickness) direction. This provided a fine mesh resolution through the channel thickness while still maintaining a reasonable overall mesh density by using anisotropically scaled cells in the width and length directions. If the Star-CCM+ mesher is tasked with meshing the As-Built geometry from scratch, it tends to struggle with the mesh scaling, and defaults to smaller sized cells. This results in a cell count that is orders of magnitude larger than the desired cell count.

Using the process outlined here, the Ideal geometry CFD background mesh was morphed using the built in Star-CCM+ mesh morphing algorithm. The resulting channel thickness profiles are shown Figure 11.1, with the black lines indicating the locations of the plate leading and trailing edges. Note that since no channel thickness data was available for the inlet and exit plenums, the measured channel geometry at the leading and trailing edges was extrapolated to the inlet and outlet boundaries. Since there is no plate present in these locations, the actual channel thickness in the plenums is in reality the sum of the thicknesses shown in Figure 11.1, plus the plate thickness of $1.016 \mathrm{~mm}$. 


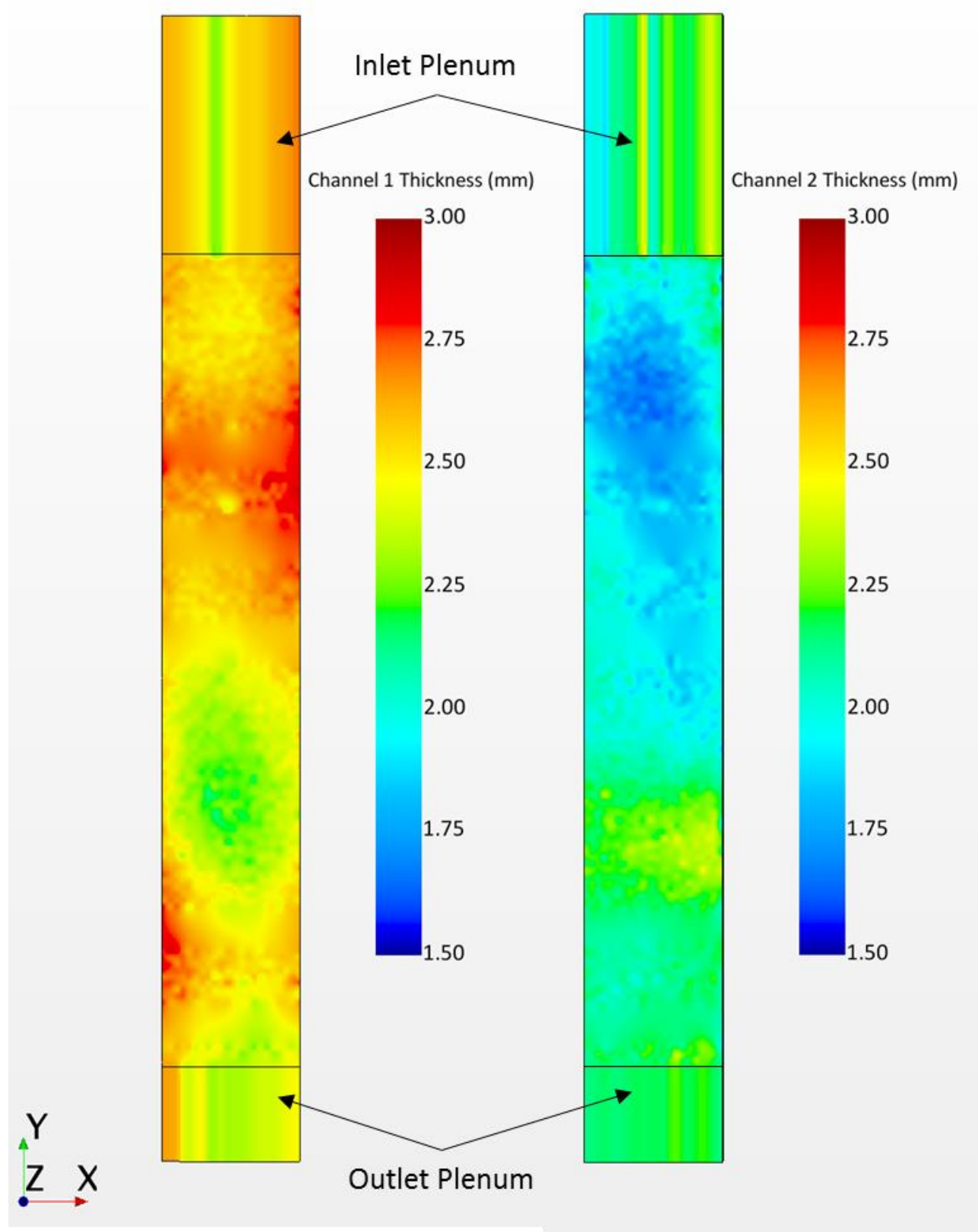

Figure 11.1. Resulting channel thickness after morphing outer fluid walls

The resulting CFD mesh maintains the same mesh density as the Ideal geometry simulations. Additionally, since the channel thickness modifications are small, the relative shape of cells to their neighbors is largely unchanged. This has the effect of 
preserving a quality grid by avoiding distorted cells and large changes in cell volume in neighboring cells.

\section{3: As-Built Geometry FSI Results}

As was done with the Ideal geometry simulations, a range of flow rates were studied with the As-Built simulations. Table 11.1 shows the flow rates, velocities, and coupling parameters used in the As-Built simulations. Note that several additional flow rates were added for these simulations. These simulations cover all of the flow rates seen in the experiment, and allow for direct comparison to the experiment.

Table 11.1. As-Built geometry FSI parameters

\begin{tabular}{ccccc}
\hline $\begin{array}{c}\text { Flow Rate } \\
(\mathbf{k g} / \mathbf{s})\end{array}$ & $\begin{array}{c}\text { Average Fluid } \\
\text { Channel Velocity } \\
(\mathbf{m} / \mathbf{s})\end{array}$ & $\begin{array}{c}\text { Coupling } \\
\text { Scheme }\end{array}$ & $\begin{array}{c}\text { Step Size } \\
(\mathbf{s})\end{array}$ & CFL \\
\hline 1.04 & 2.07 & Explicit & $6.35 \mathrm{E}-3$ & 10.34 \\
1.40 & 2.78 & Explicit & $4.23 \mathrm{E}-3$ & 9.27 \\
1.74 & 3.46 & Explicit & $1.59 \mathrm{E}-3$ & 4.33 \\
2.30 & 4.57 & Explicit & $1.27 \mathrm{E}-3$ & 4.57 \\
2.60 & 5.17 & Explicit & $1.20 \mathrm{E}-3$ & 4.88 \\
2.76 & 5.49 & Explicit & $1.13 \mathrm{E}-3$ & 4.88 \\
2.86 & 5.69 & Explicit & $9.27 \mathrm{E}-4$ & 4.15 \\
2.95 & 5.86 & Explicit & $8.75 \mathrm{E}-4$ & 4.04 \\
3.43 & 6.82 & Implicit & $6.62 \mathrm{E}-4$ & 3.55 \\
3.91 & 7.77 & Implicit & $5.20 \mathrm{E}-4$ & 3.18 \\
4.34 & 8.63 & Implicit & $4.28 \mathrm{E}-4$ & 2.91 \\
\hline & & & & \\
\hline & 5 & & & \\
\hline
\end{tabular}


For each of the flow rates in Table 11.1, the final axial deflection profile of the plate was extracted from the Abaqus results. These axial deflection profiles, shown in Figure 11.2, are substantially different from the Ideal geometry profiles of Figure 10.4. Notably, the peak deflection is significantly higher. Additionally, some regions of the plate deflected into the smaller channel. One similarity seems to be the development of additional bubbles in the deflection profile near the trailing edge of the plate.

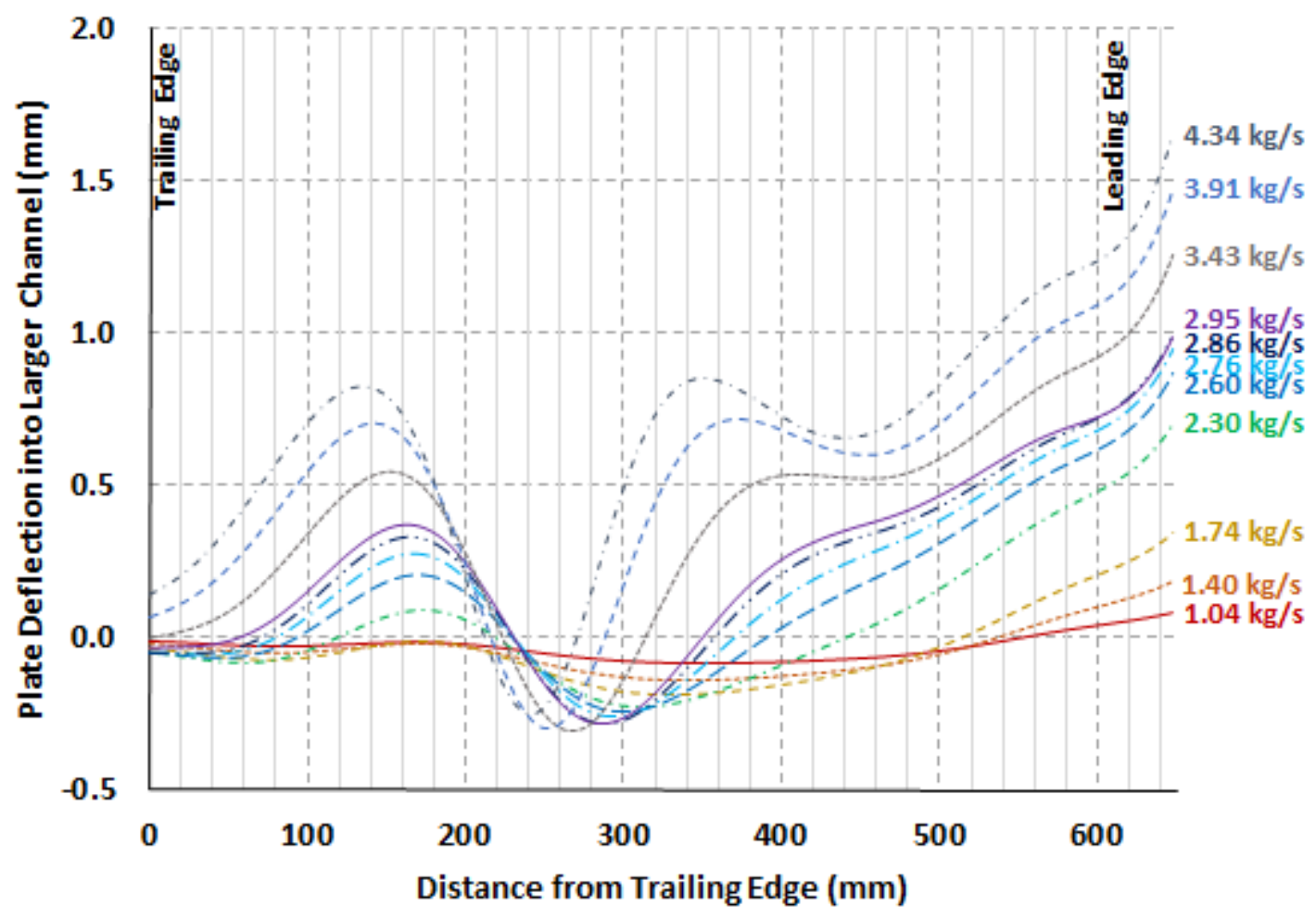

Figure 11.2. Axial deflection profiles for As-Built geometry FSI simulations

To determine the pressure force acting on the plate for each simulation, the pressure in the two fluid channels was extracted and the difference taken along the axial centerline of the model. The resulting data of Figure 11.3 show predominately larger pressure in the 
smaller fluid channel, which corresponds well with the plate deflection into the larger shown in Figure 11.2.

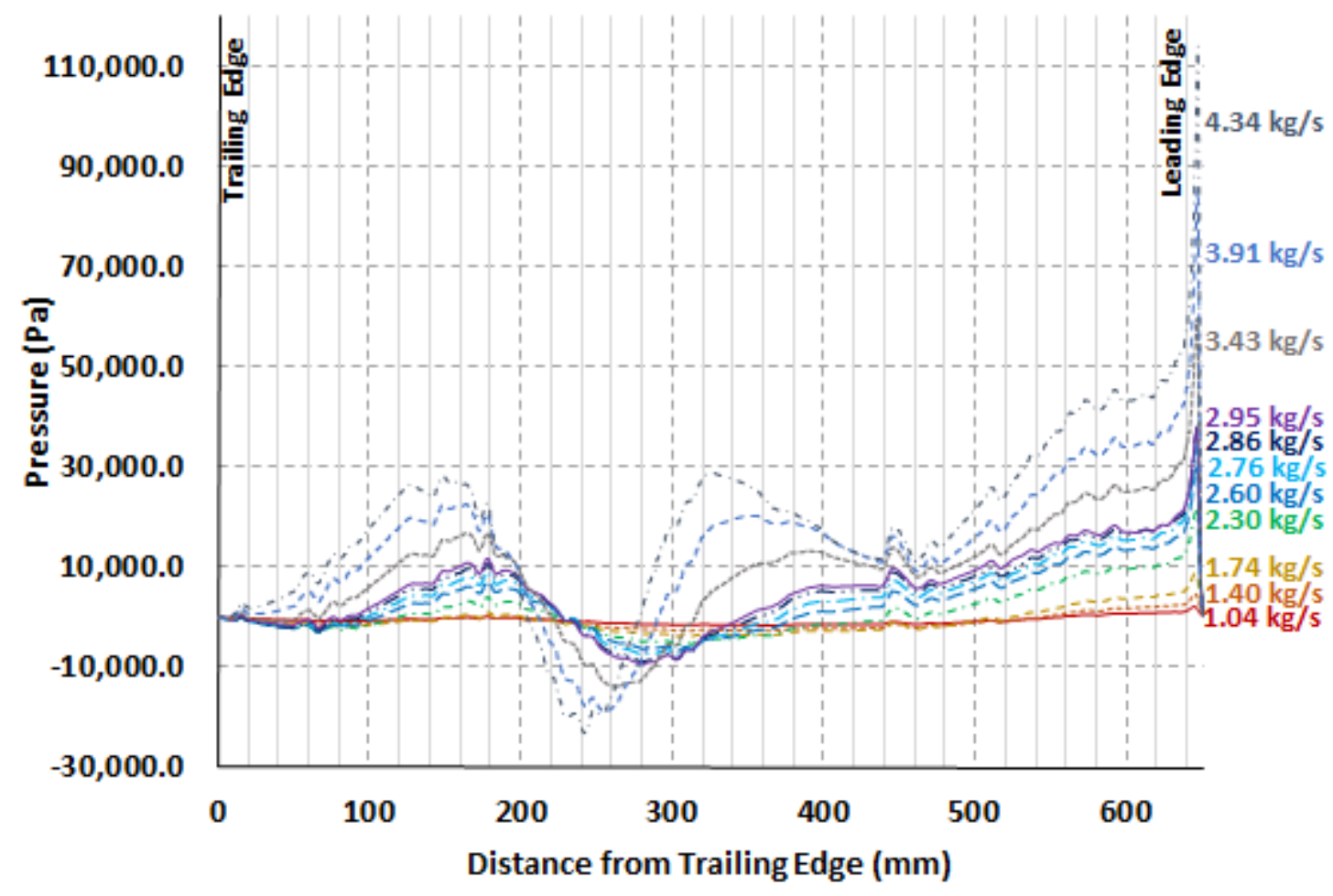

Figure 11.3. Axial pressure profiles for As-Built FSI simulations 


\section{CHAPTER 12: COMPARISONS \& CONCLUSIONS}

\section{1: Deflection Results Comparison}

The ultimate goal of developing the As-Built modeling technique was to more accurately capture the experiment results. If the numeric models can be shown to match the experiment, then the numeric modeling technique can be validated. Initial efforts to model the experiment geometry using idealized dimensions failed to capture important aspects of the problem. By modifying the CFD mesh to more accurately represent the fluid channel geometry in the experiment, the simulations should more closely match reality. Figure 12.1 through Figure 12.11 show the axial deflection comparison between the Experiment results and the As-Built FSI simulations. Note that the experiment deflection results are the average measured deflection from both laser displacement sensors, and the error bars indicate the $95 \%$ confidence interval which encompasses both sets of laser data. Additionally, results from the Ideal geometry FSI simulations are included where available.

From section 9.3, the FEA deflection results were found to match the analytic Timoshenko solution to within $0.58 \%$. If a factor of safety of 1.5 is used, the uncertainty of the numeric deflection results would be $\pm 0.87 \%$. 

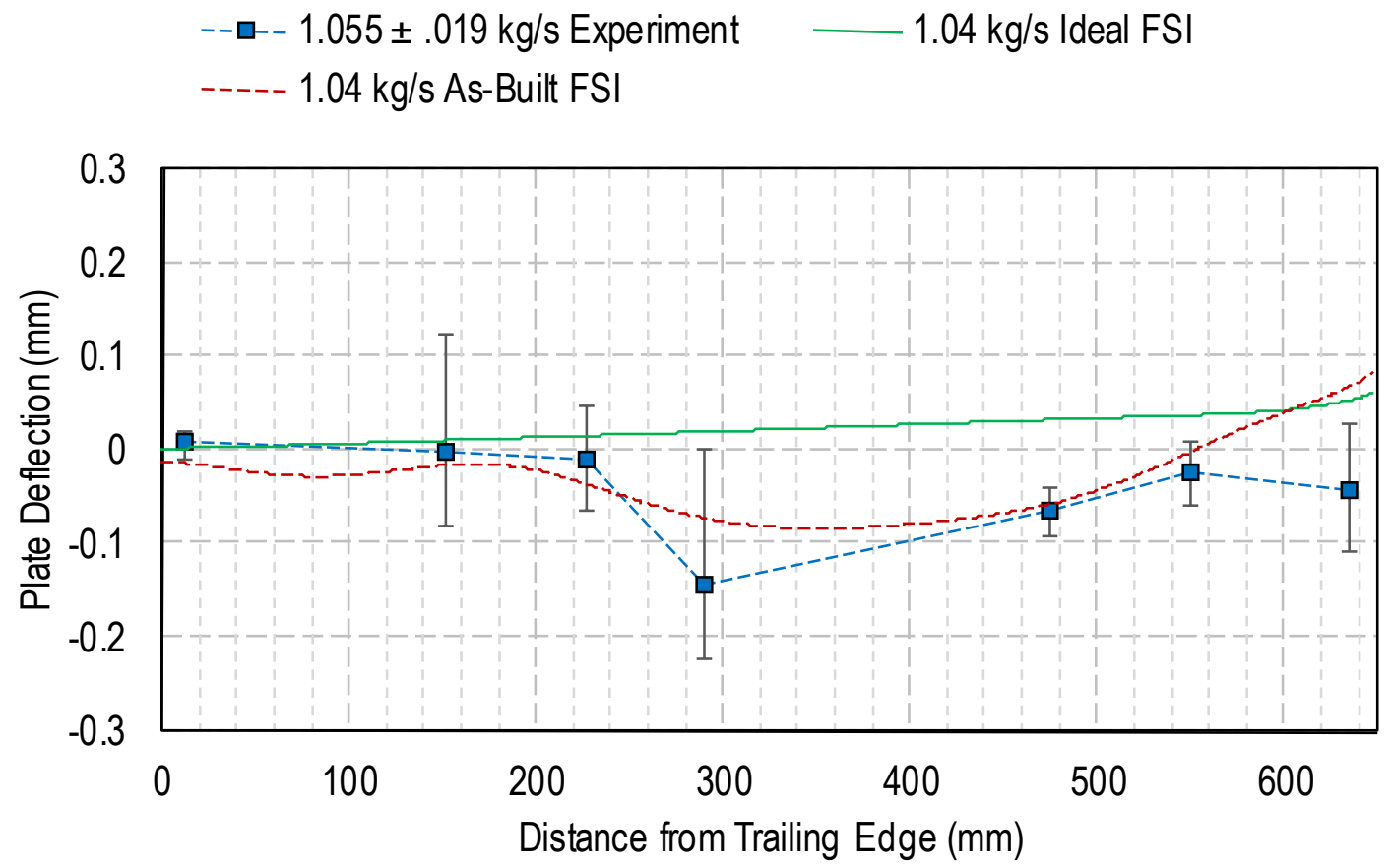

Figure 12.1. $1.04 \mathrm{~kg} / \mathrm{s}$ experiment \& FSI axial deflection results comparison

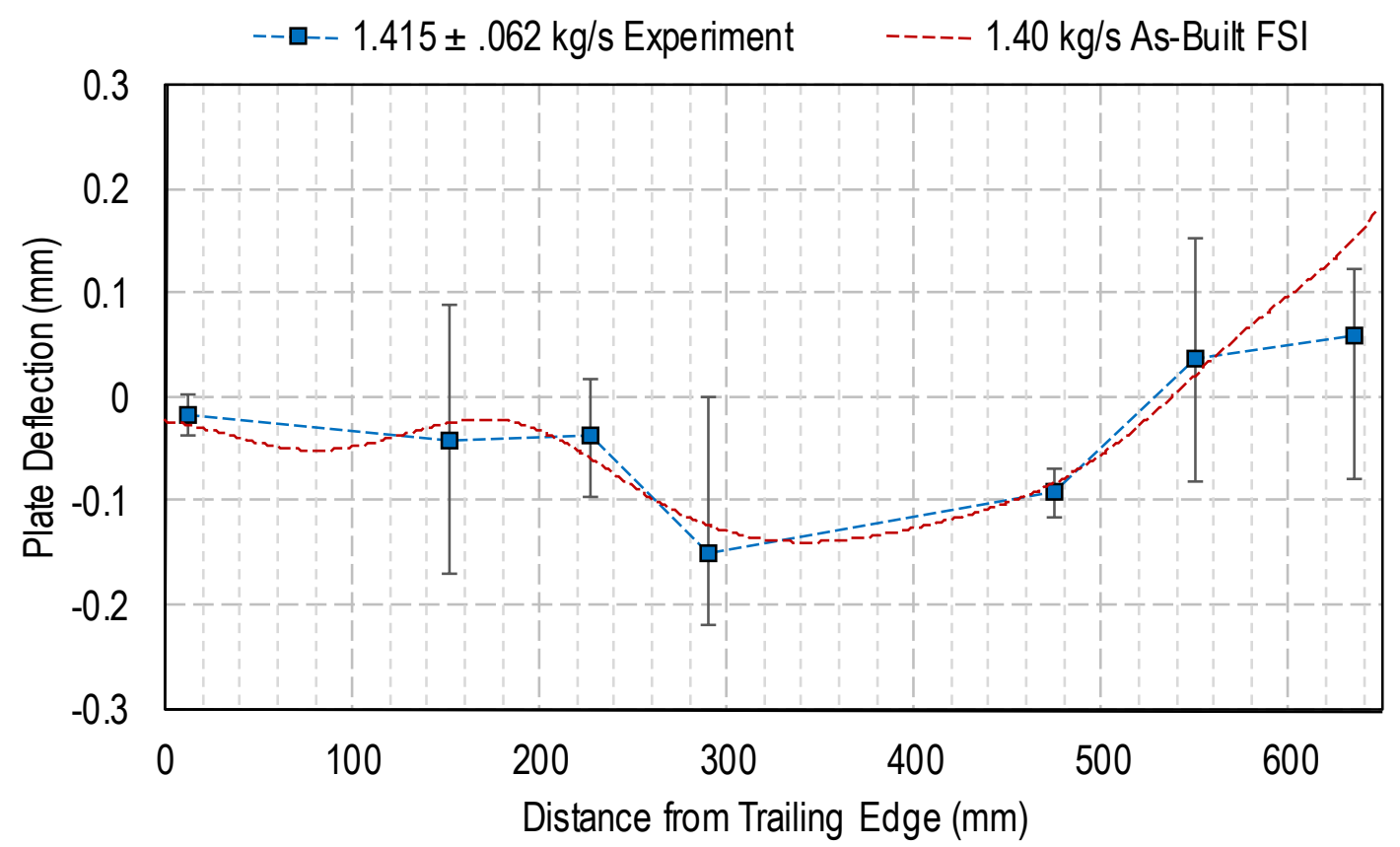

Figure 12.2. $1.40 \mathrm{~kg} / \mathrm{s}$ experiment \& FSI axial deflection results comparison 
--- $1.763 \pm .100 \mathrm{~kg} / \mathrm{s}$ Experiment $1.74 \mathrm{~kg} / \mathrm{s}$ Ideal FSI

\section{$1.74 \mathrm{~kg} / \mathrm{s}$ As-Built FSI}

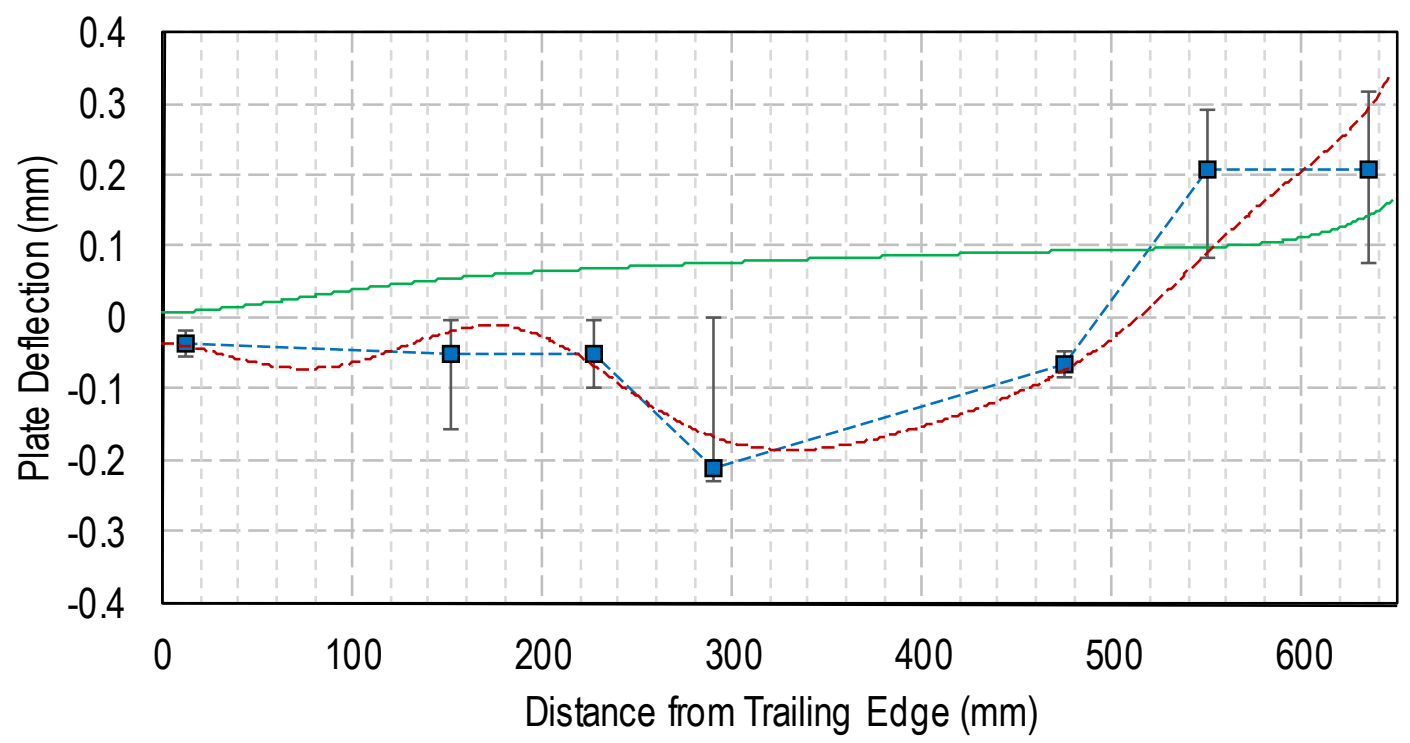

Figure 12.3. $1.74 \mathrm{~kg} / \mathrm{s}$ experiment \& FSI axial deflection results comparison
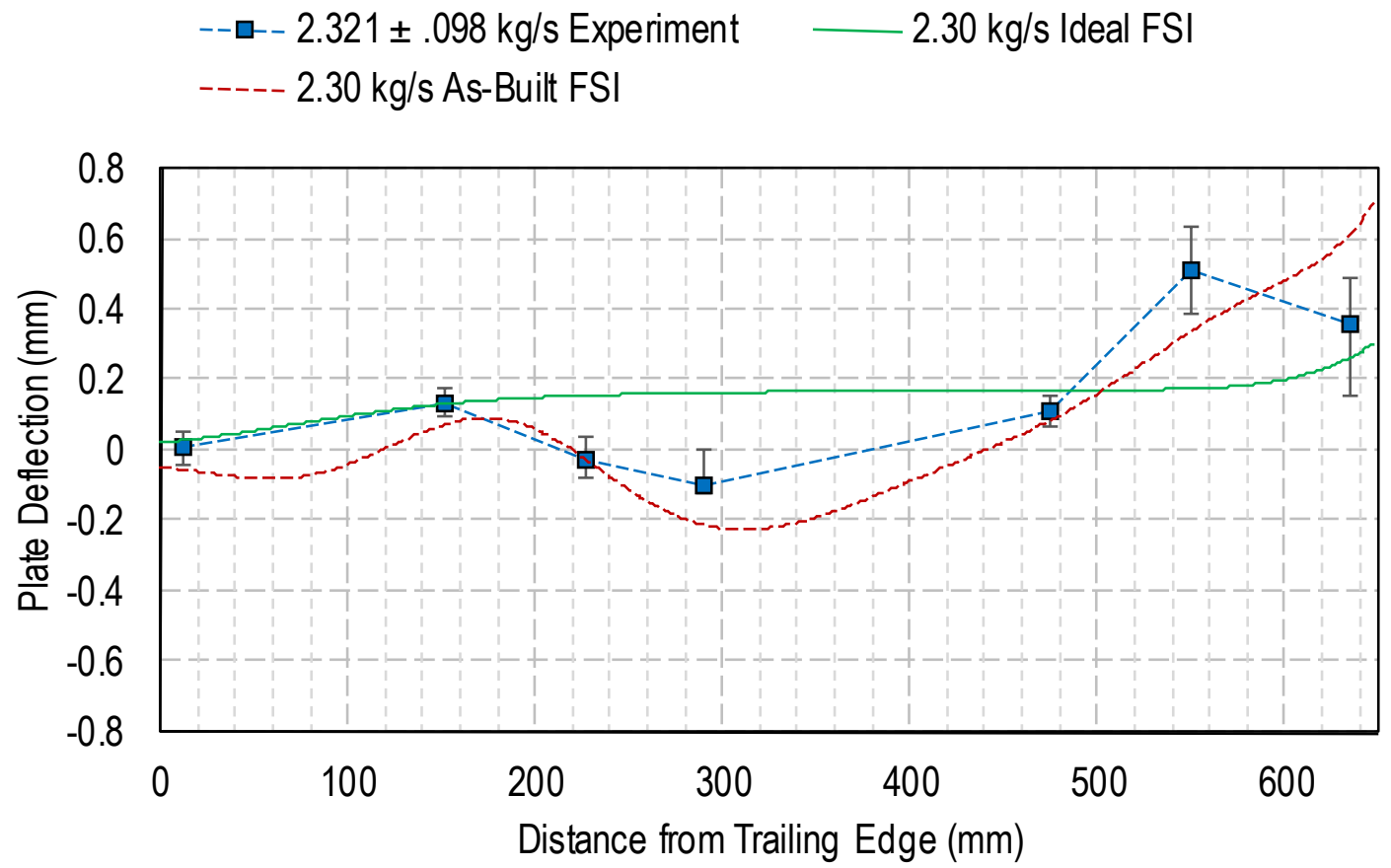

Figure 12.4. $2.30 \mathrm{~kg} / \mathrm{s}$ experiment \& FSI axial deflection results comparison 

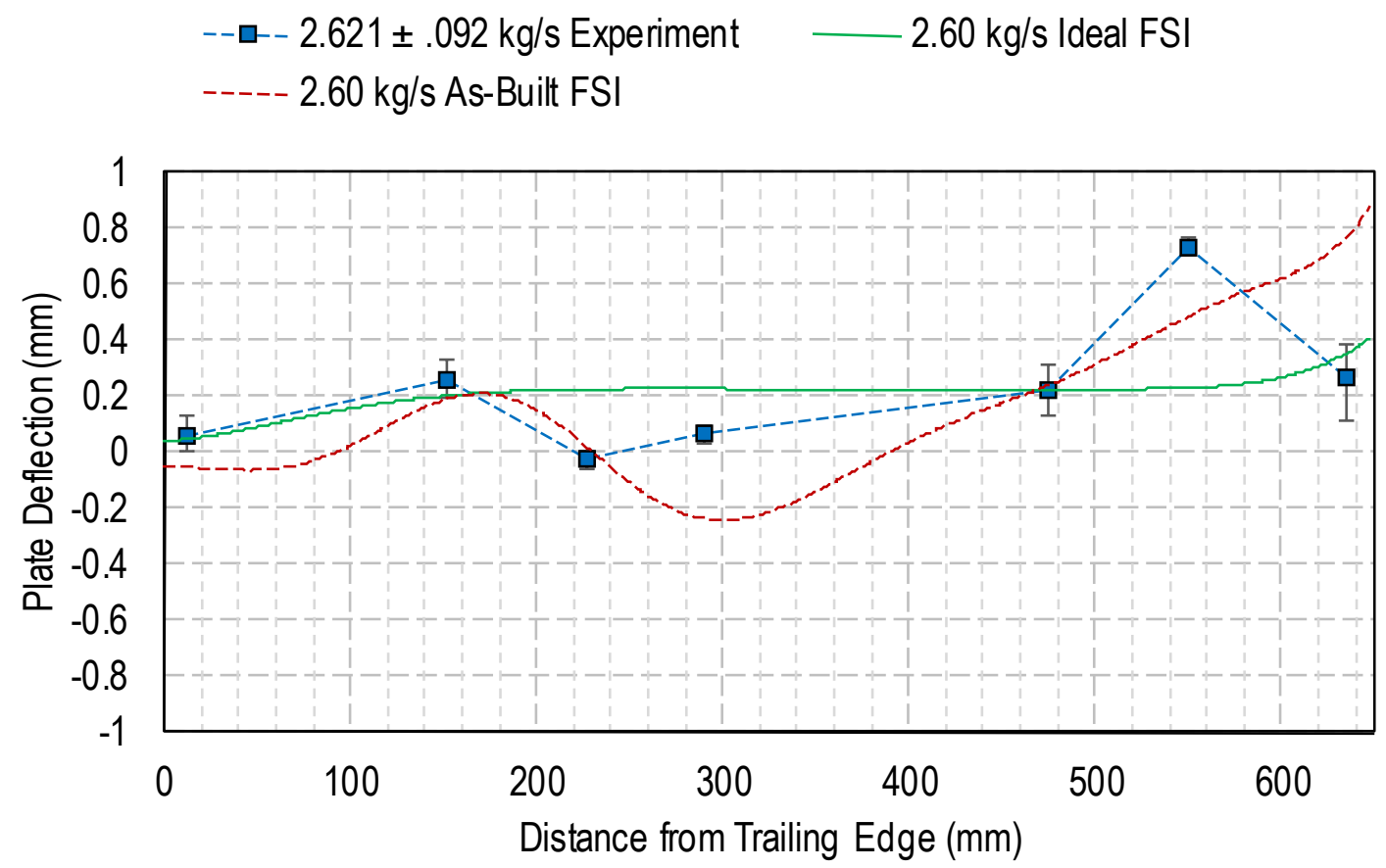

Figure 12.5. $2.60 \mathrm{~kg} / \mathrm{s}$ experiment \& FSI axial deflection results comparison

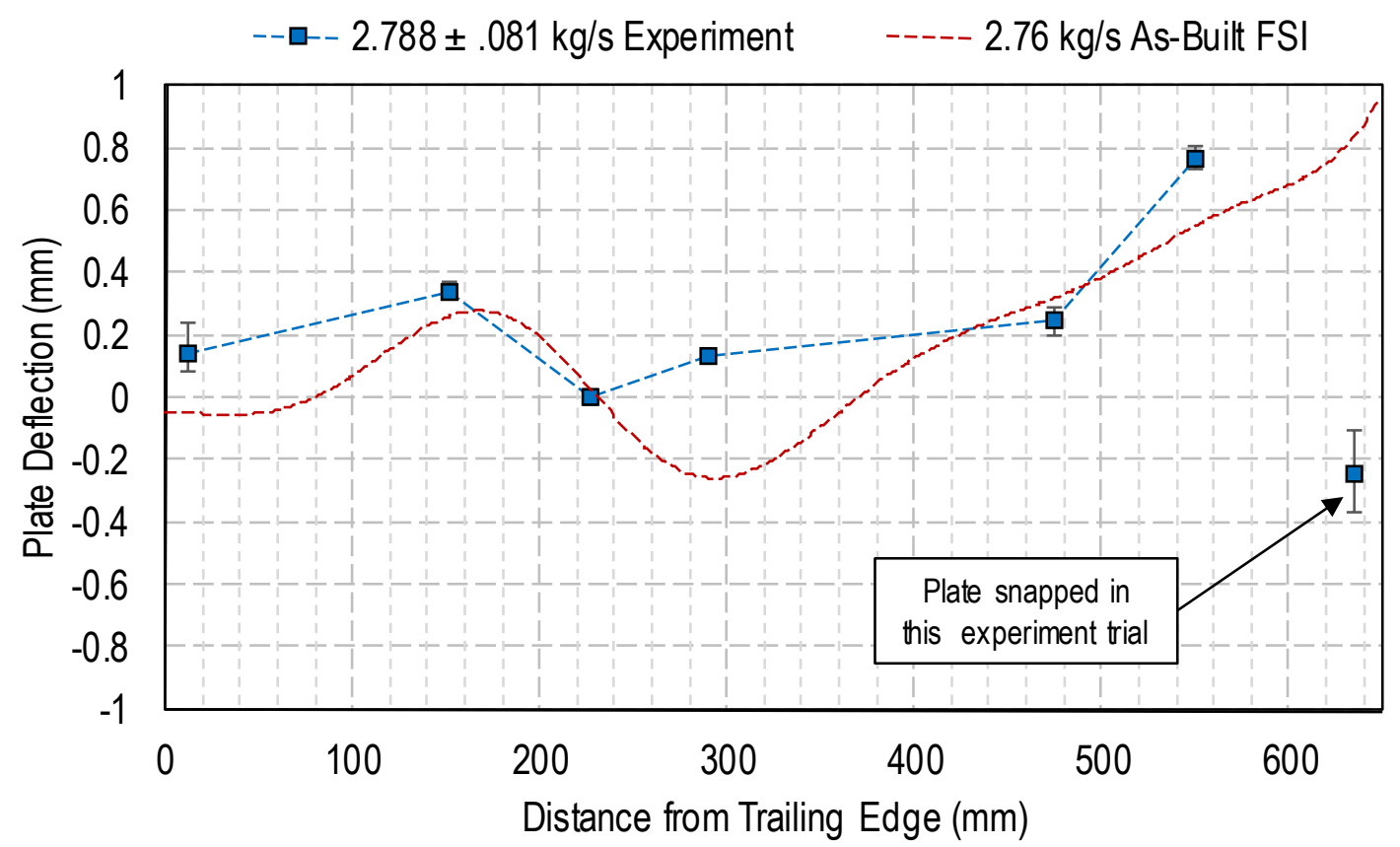

Figure 12.6. $2.76 \mathrm{~kg} / \mathrm{s}$ experiment \& FSI axial deflection results comparison 
---- $2.883 \pm .095 \mathrm{~kg} / \mathrm{s}$ Experiment

$2.86 \mathrm{~kg} / \mathrm{s}$ Ideal FSI

----- $2.86 \mathrm{~kg} / \mathrm{s}$ As-Built FSI

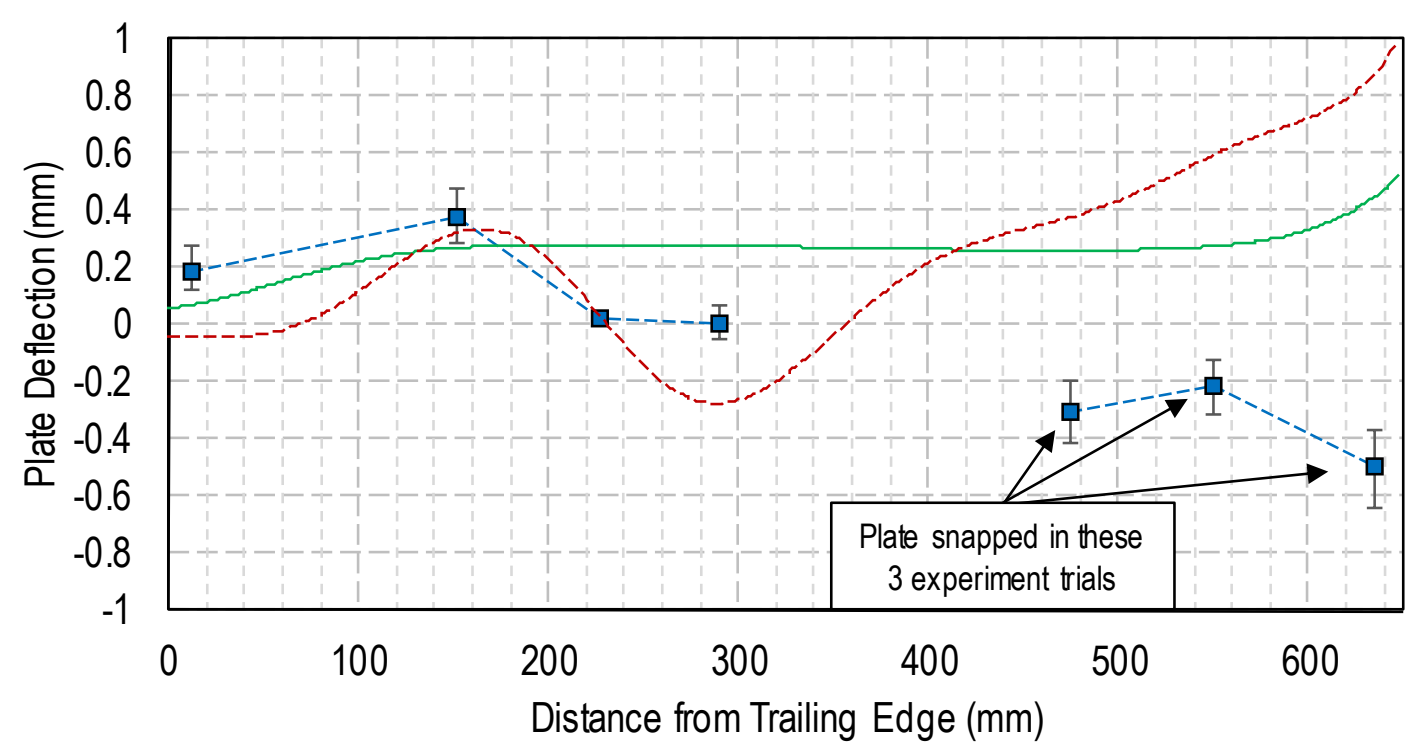

Figure 12.7. $2.86 \mathrm{~kg} / \mathrm{s}$ experiment \& FSI axial deflection results comparison

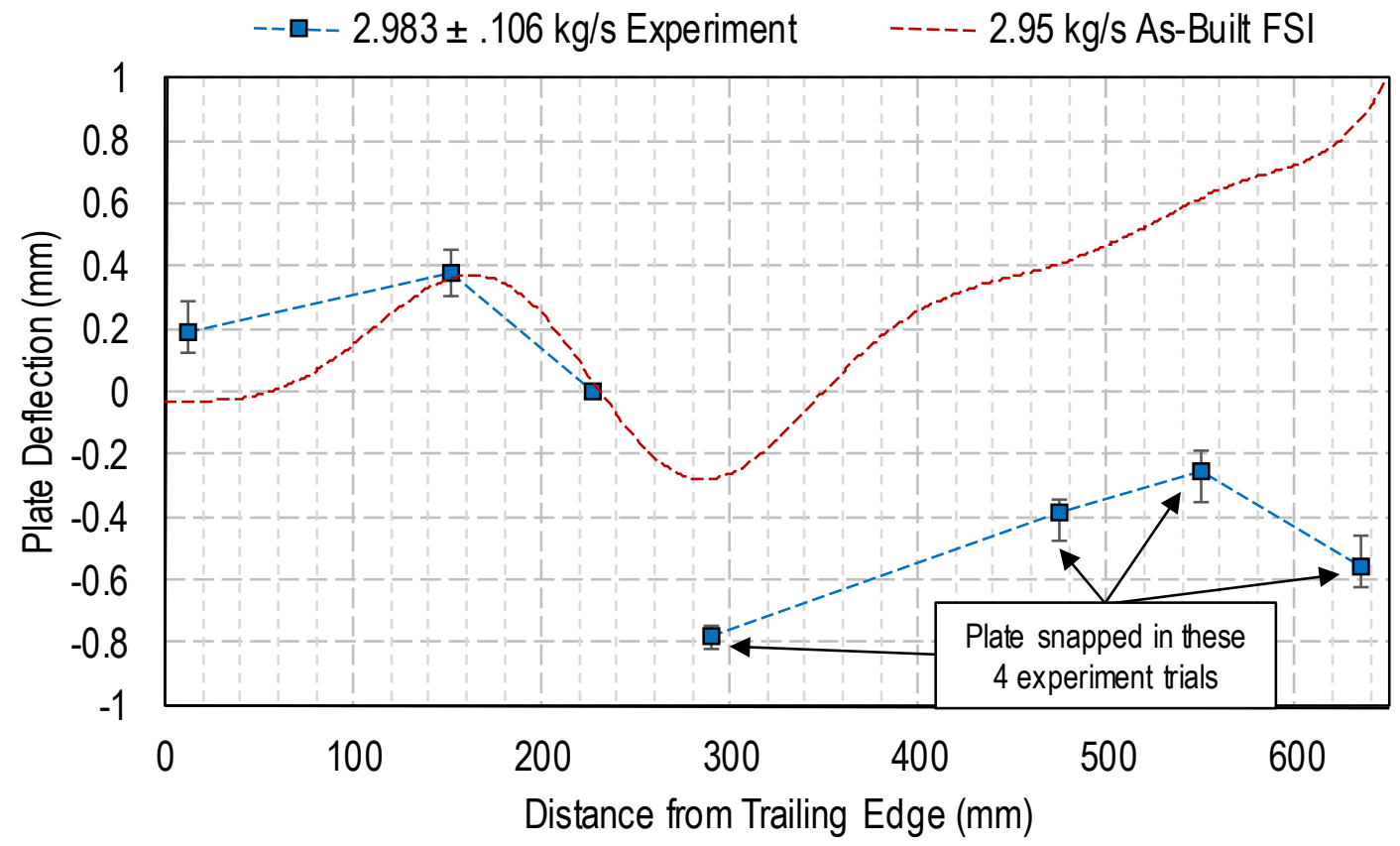

Figure 12.8. $2.95 \mathrm{~kg} / \mathrm{s}$ experiment \& FSI axial deflection results comparison 

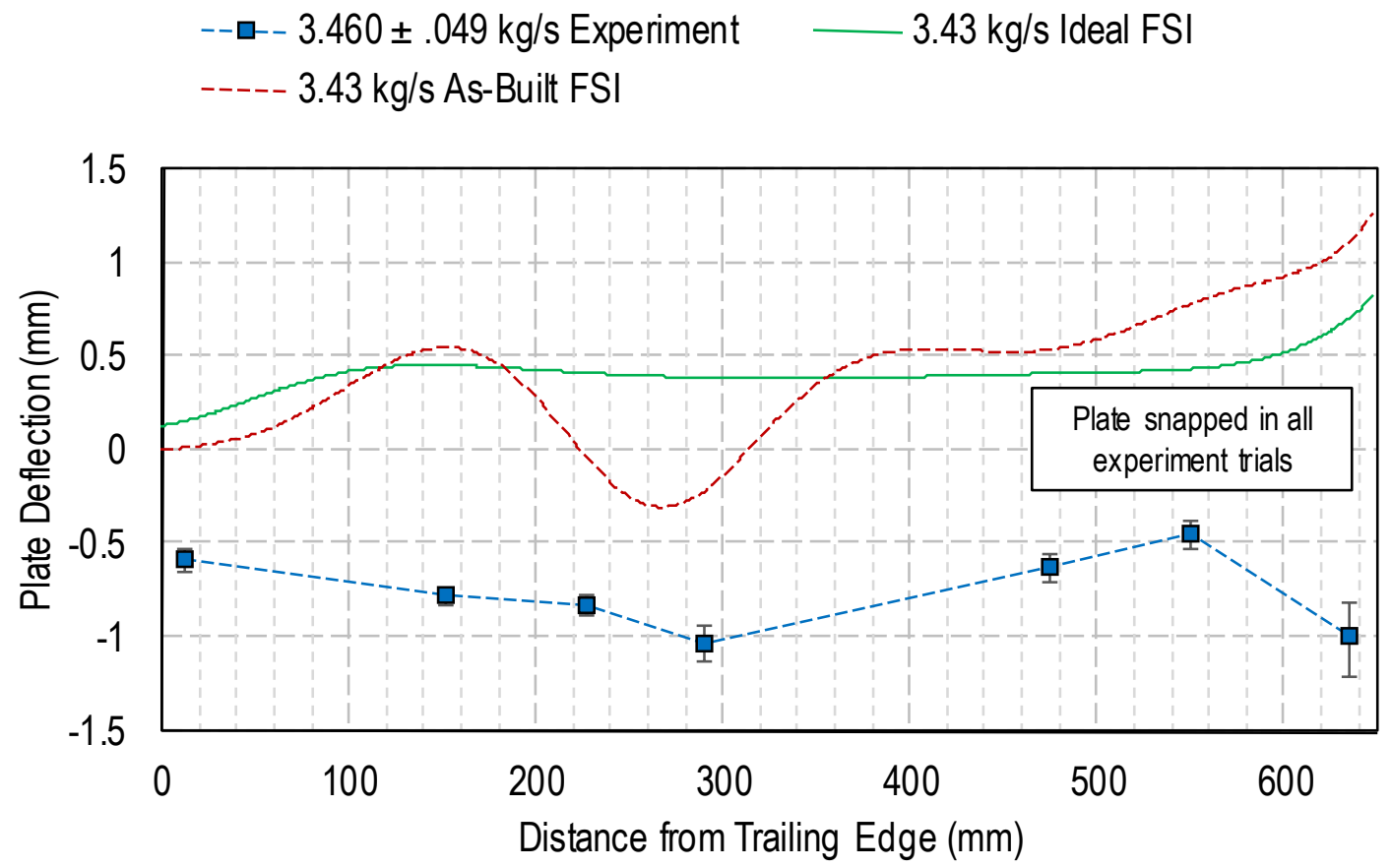

Figure 12.9. $3.43 \mathrm{~kg} / \mathrm{s}$ experiment \& FSI axial deflection results comparison

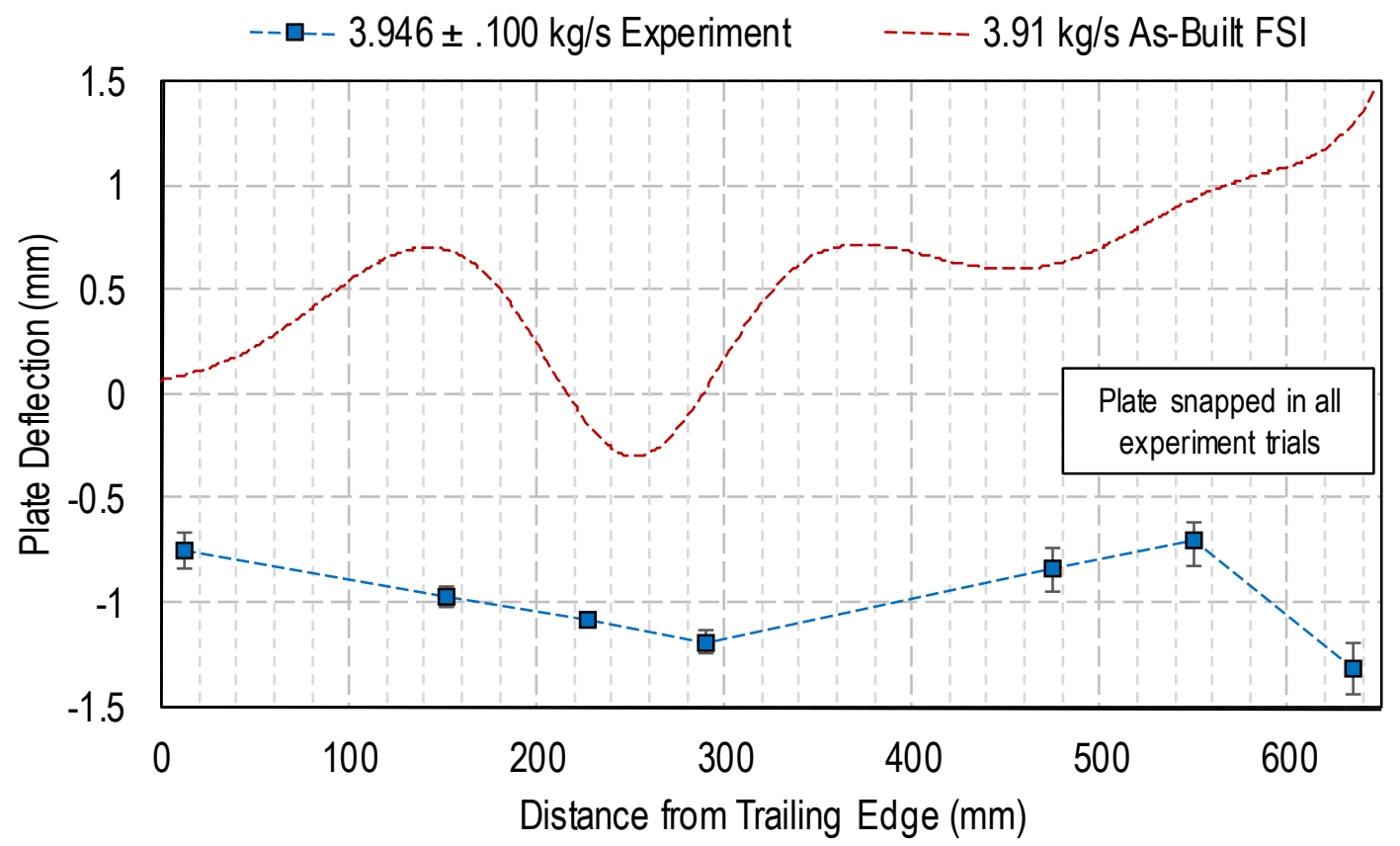

Figure 12.10. $3.91 \mathrm{~kg} / \mathrm{s}$ experiment \& FSI axial deflection results comparison 
$--\square-4.376 \pm .093 \mathrm{~kg} / \mathrm{s}$ Experiment

$4.34 \mathrm{~kg} / \mathrm{s}$ Ideal FSI

$4.34 \mathrm{~kg} / \mathrm{s}$ As-Built FSI

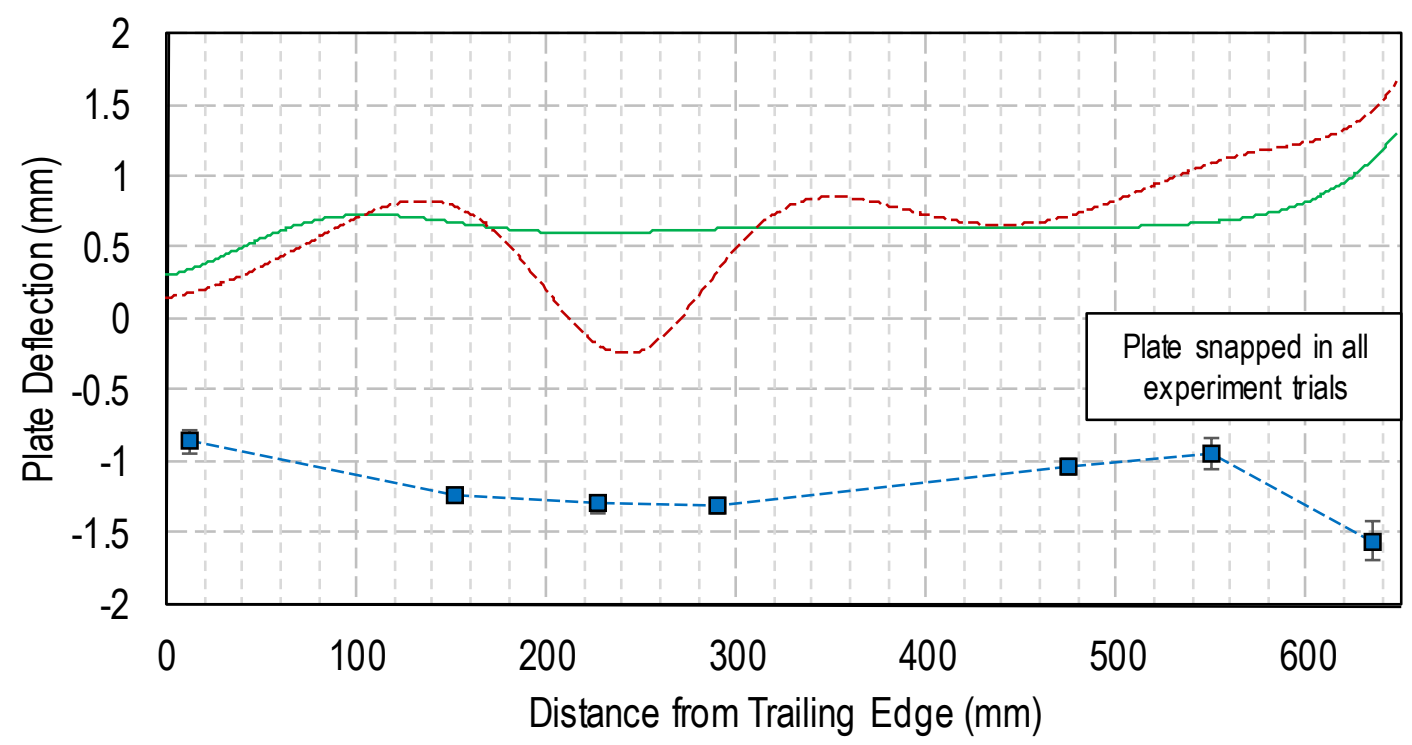

Figure 12.11. $4.34 \mathrm{~kg} / \mathrm{s}$ experiment \& FSI axial deflection results comparison

In nearly all cases, the As-Built FSI simulation closely matches the deflections observed in the experiment pre-snap cases. Particularly when compared to the Ideal geometry results, the As-Built simulations are a marked improvement in capturing the actual plate deflection. For these lower to mid-range velocities where no plate snap was observed, the numeric models do an excellent job of predicting plate deflection.

At higher flow rates, the experiments observed a sudden plate 'snap', where the plate sudden changed from a static deflection into the larger channel to a static deflection into the smaller channel. This deflection continued into the smaller channel and was seen in every experiment trial, although the precise flow rate fluctuated a bit. A more detailed analysis of the plate 'snap' is available in sections 5.3.4 \& 5.3.5. While the As-Built models significantly improved in their matching of the pre-snap deflection profile, no 
plate snap was observed in any FSI simulations. Therefore, at higher flow rates where the plate snapped in the experiment, the matching between the experiment results and the As-Built FSI models is quite poor.

\section{2: Pressure Results Comparison}

In addition to the plate deflection results, the pressure force acting on the plate is also of interest. In order to examine the impact the As-Built geometry had on the prediction of the pressure force acting on the plate, the pressure differential data from the experiment, Ideal geometry simulations, and As-Built geometry simulations has been plotted at all 11 flow rates of interest. The pressure data was calculated by taking the difference between the pressure in the smaller and larger channels along the axial centerline of the geometry. Positive pressure indicates higher pressure in the smaller channel, tending to push the plate into the larger channel. Since the pressure data collection in the experiment was repeated seven times, the average of all seven repetitions was used in creating the pressure comparison plots. Finally, since some experiment trials saw the plate snap while other didn't, the pressure data has been segregated into pre- and post-snap series. Since the numeric models did not experience a snap, it is appropriate to compare the pre-snap experiment pressure to the numeric model pressure results. The plots for all 11 flow rates are shown in Figure 12.12 through Figure 12.22. 
$--\square-1.055 \pm .019 \mathrm{~kg} / \mathrm{s}$ Pre-Snap Expt. $1.04 \mathrm{~kg} / \mathrm{s}$ Ideal FSI $1.04 \mathrm{~kg} / \mathrm{s}$ As-Built FSI

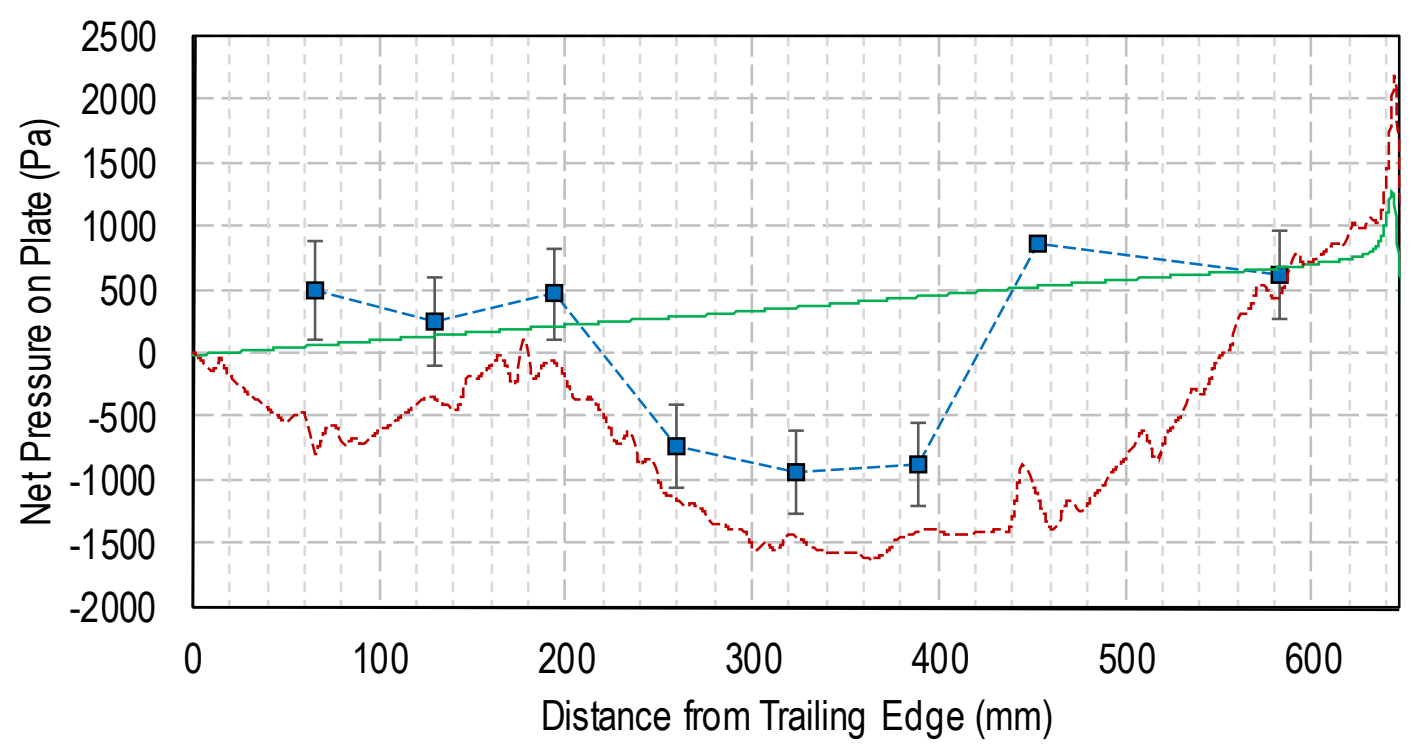

Figure 12.12. $1.04 \mathrm{~kg} / \mathrm{s}$ experiment \& FSI axial pressure results comparison

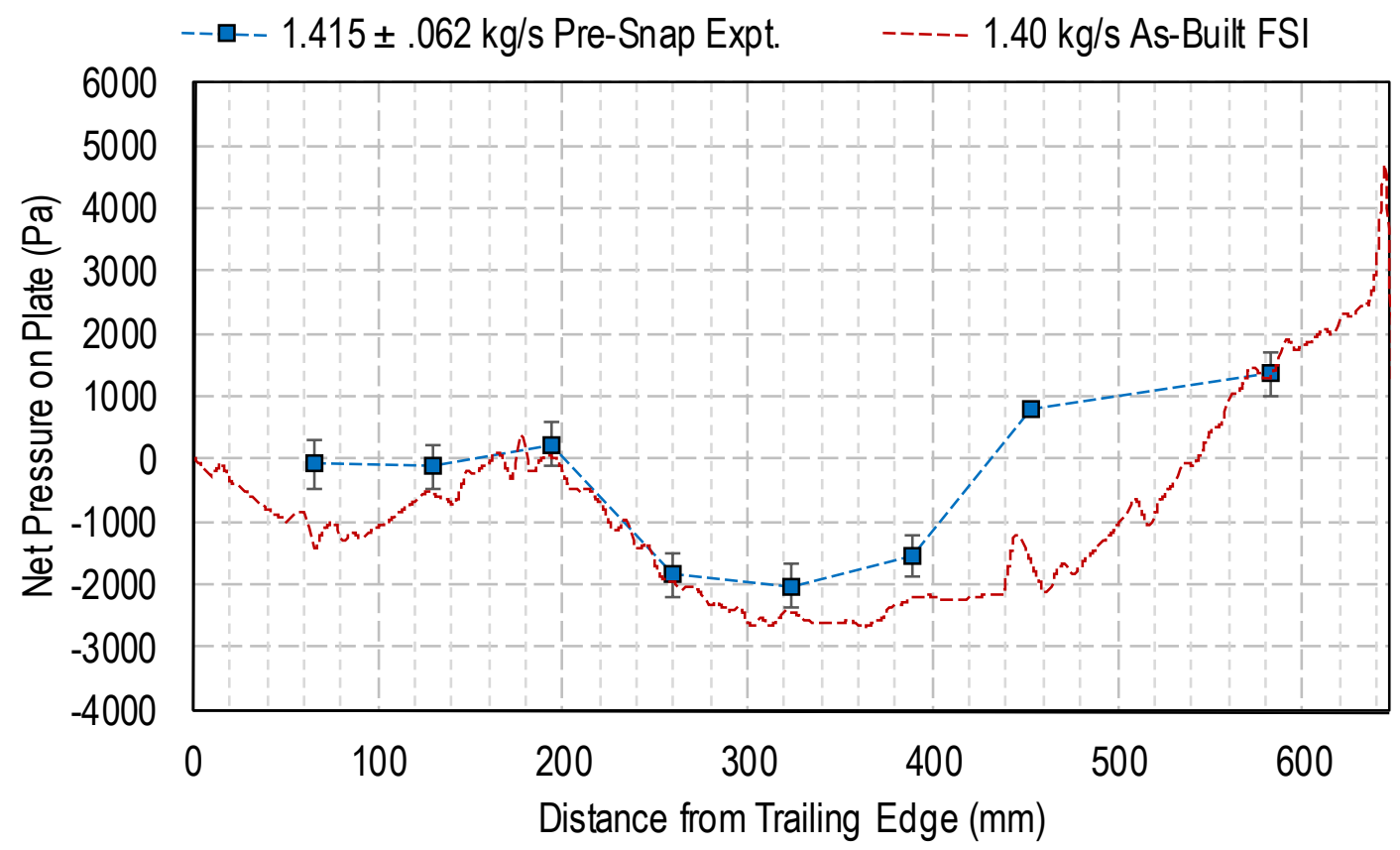

Figure 12.13. $1.40 \mathrm{~kg} / \mathrm{s}$ experiment \& FSI axial pressure results comparison 
$--\square-1.763 \pm .100 \mathrm{~kg} / \mathrm{s}$ Pre-Snap Expt.

$1.74 \mathrm{~kg} / \mathrm{s}$ As-Built FSI

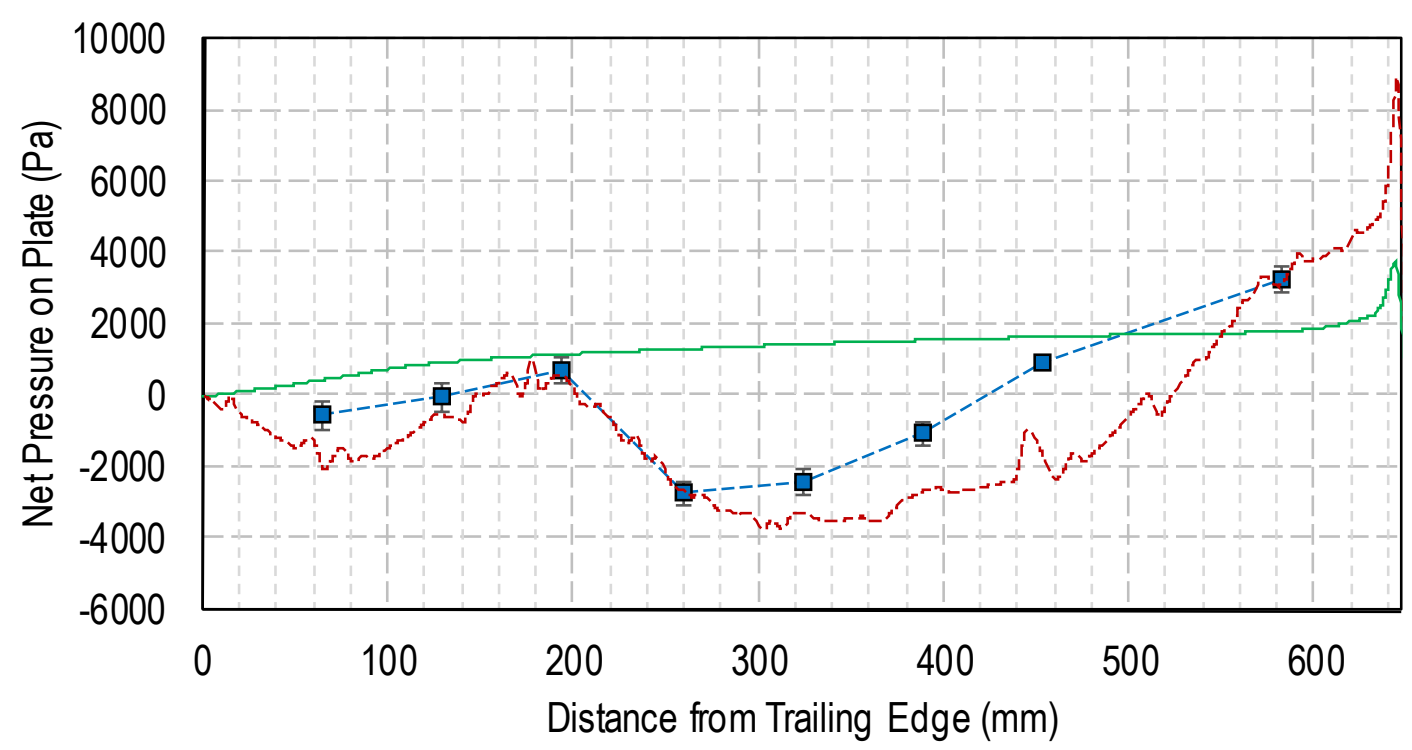

Figure 12.14. $1.74 \mathrm{~kg} / \mathrm{s}$ experiment \& FSI axial pressure results comparison

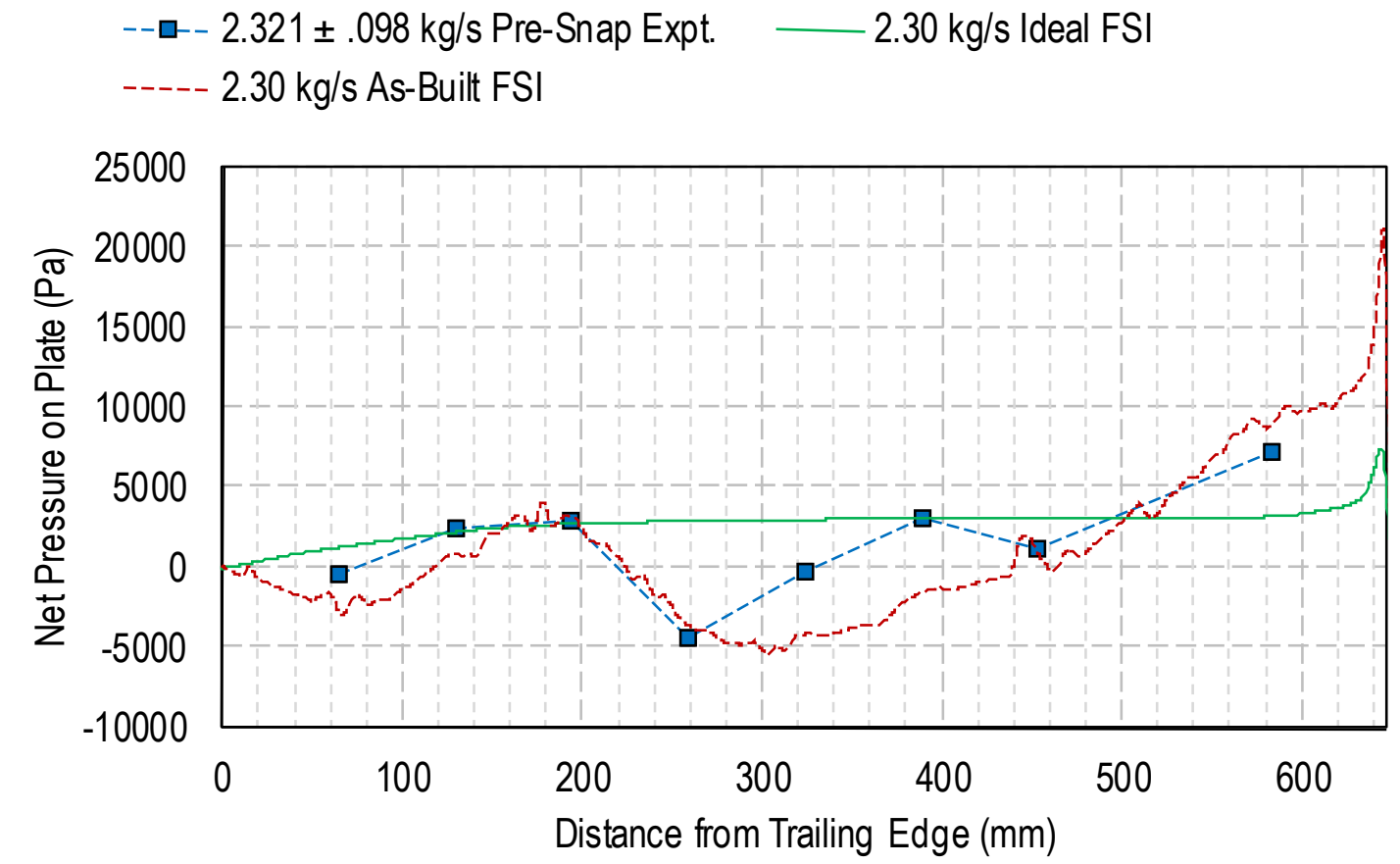

Figure 12.15. $2.30 \mathrm{~kg} / \mathrm{s}$ experiment \& FSI axial pressure results comparison 
-- $-2.621 \pm .092 \mathrm{~kg} / \mathrm{s}$ Pre-Snap Expt.

$-2.60 \mathrm{~kg} / \mathrm{s}$ Ideal FSI

----- $2.60 \mathrm{~kg} / \mathrm{s}$ As-Built FSI

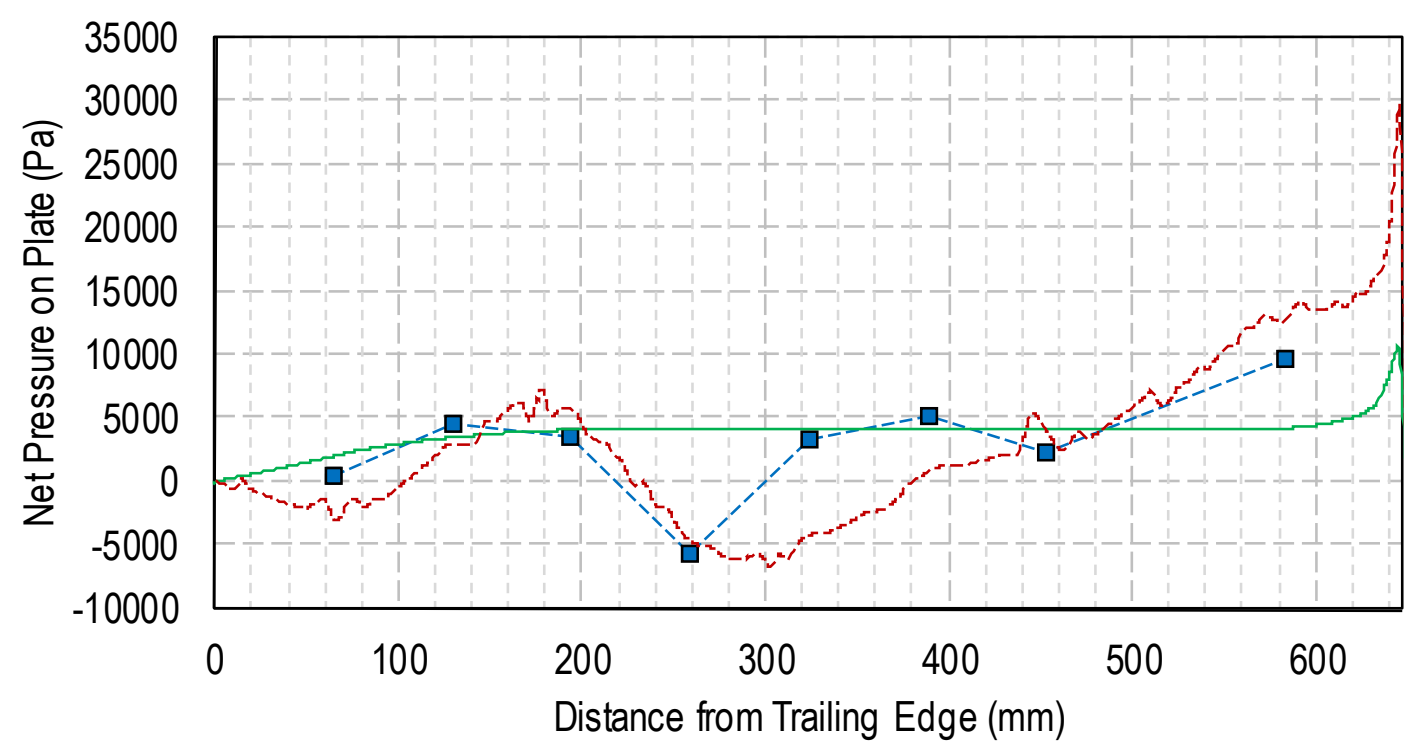

Figure 12.16. $2.60 \mathrm{~kg} / \mathrm{s}$ experiment \& FSI axial pressure results comparison

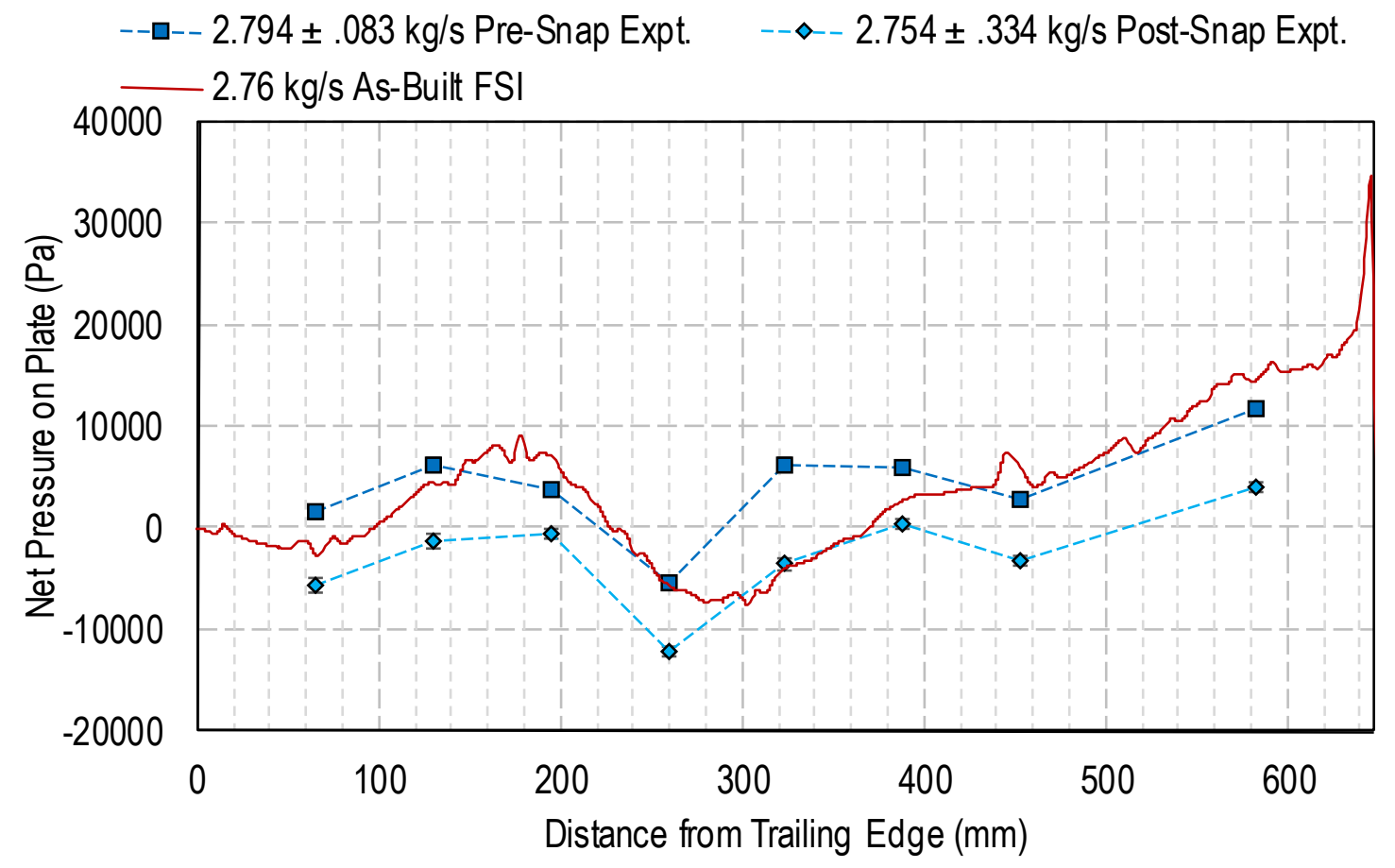

Figure 12.17. $2.76 \mathrm{~kg} / \mathrm{s}$ experiment \& FSI axial pressure results comparison 
$--\square-2.874 \pm .104 \mathrm{~kg} / \mathrm{s}$ Pre-Snap Expt. $\quad--\diamond-2.895 \pm .077 \mathrm{~kg} / \mathrm{s}$ Post-Snap Expt. $2.86 \mathrm{~kg} / \mathrm{s}$ Ideal FSI $2.86 \mathrm{~kg} / \mathrm{s}$ As-Built FSI

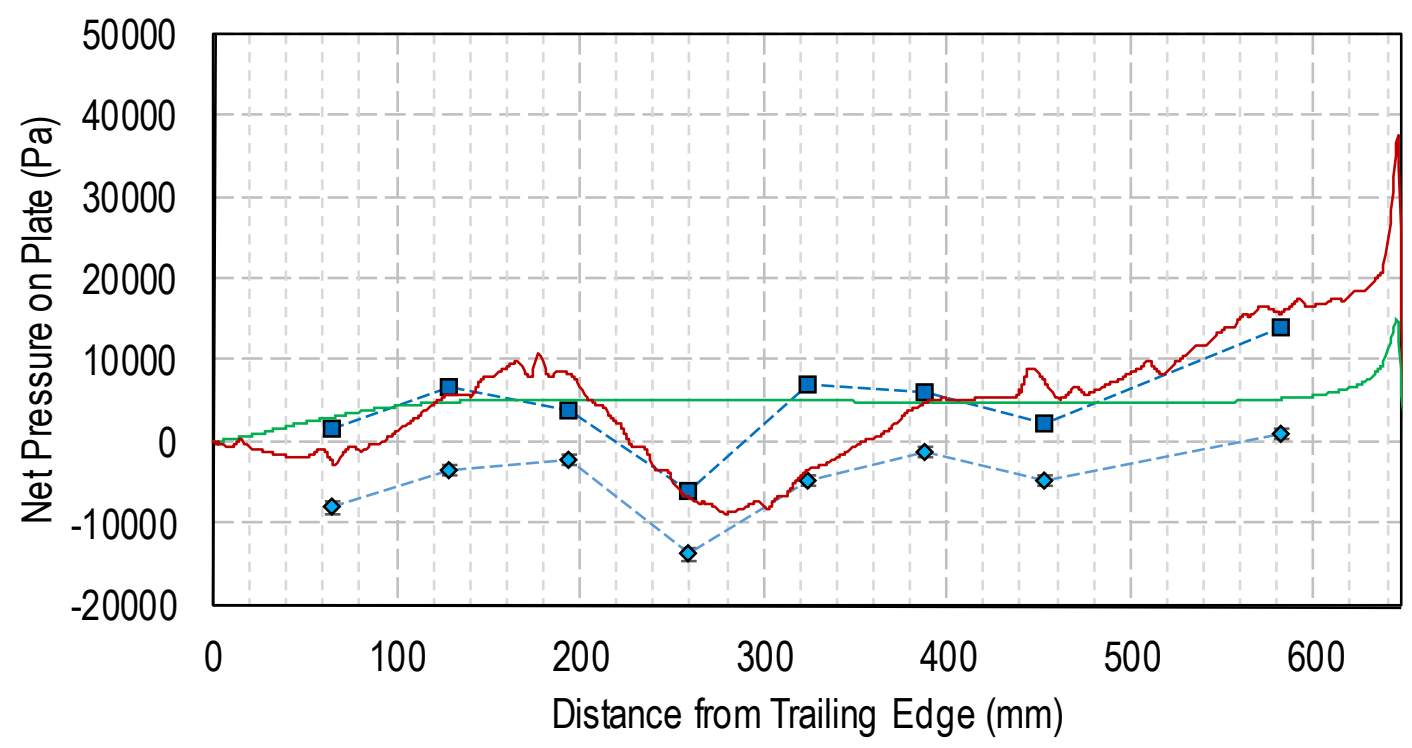

Figure 12.18. $2.86 \mathrm{~kg} / \mathrm{s}$ experiment \& FSI axial pressure results comparison

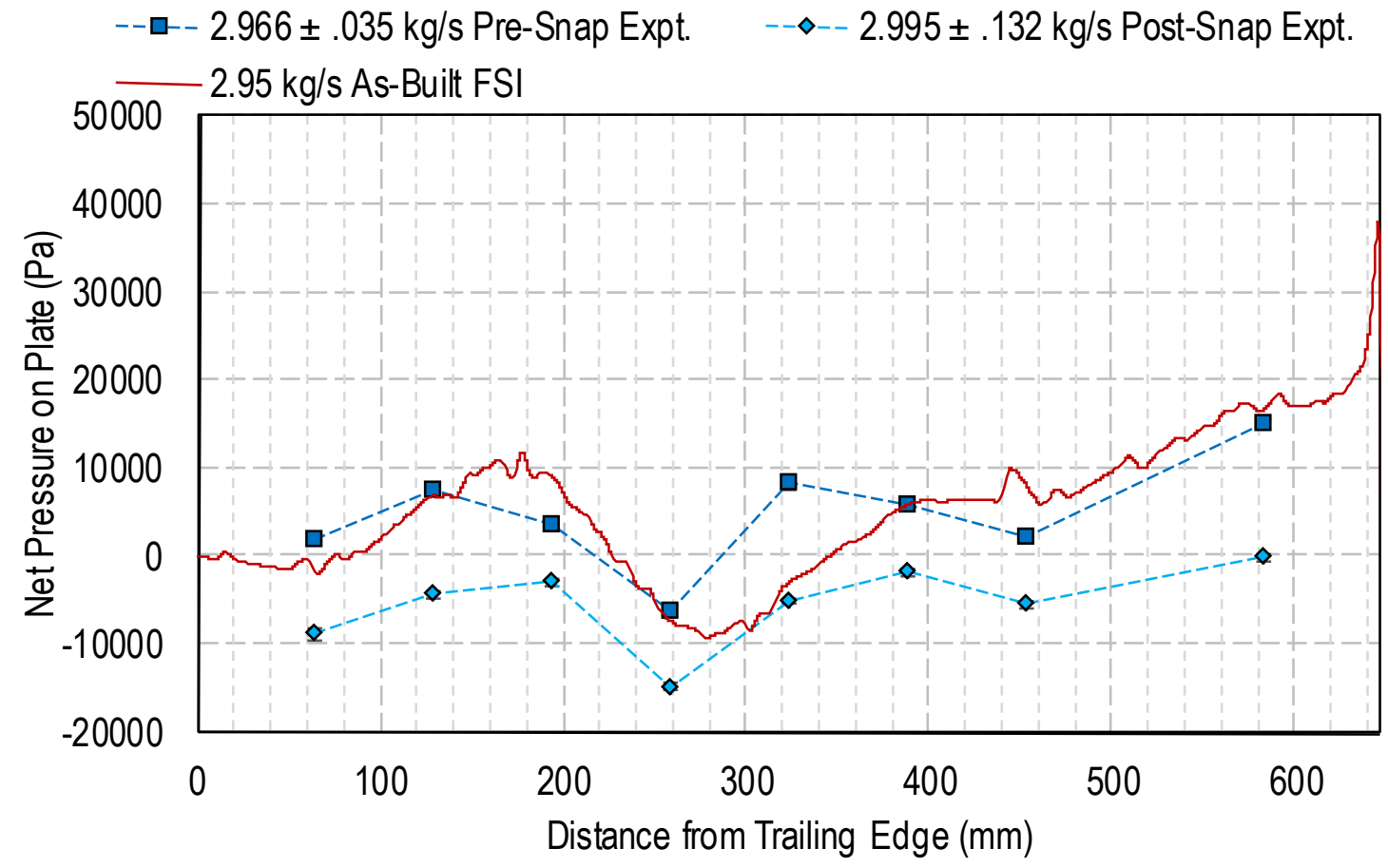

Figure 12.19. $2.95 \mathrm{~kg} / \mathrm{s}$ experiment \& FSI axial pressure results comparison 
$--\diamond--3.460 \pm .049$ kg/s Post-Snap Expt.

$3.43 \mathrm{~kg} / \mathrm{s}$ Ideal FSI

\section{$3.43 \mathrm{~kg} / \mathrm{s}$ As-Built FSI}

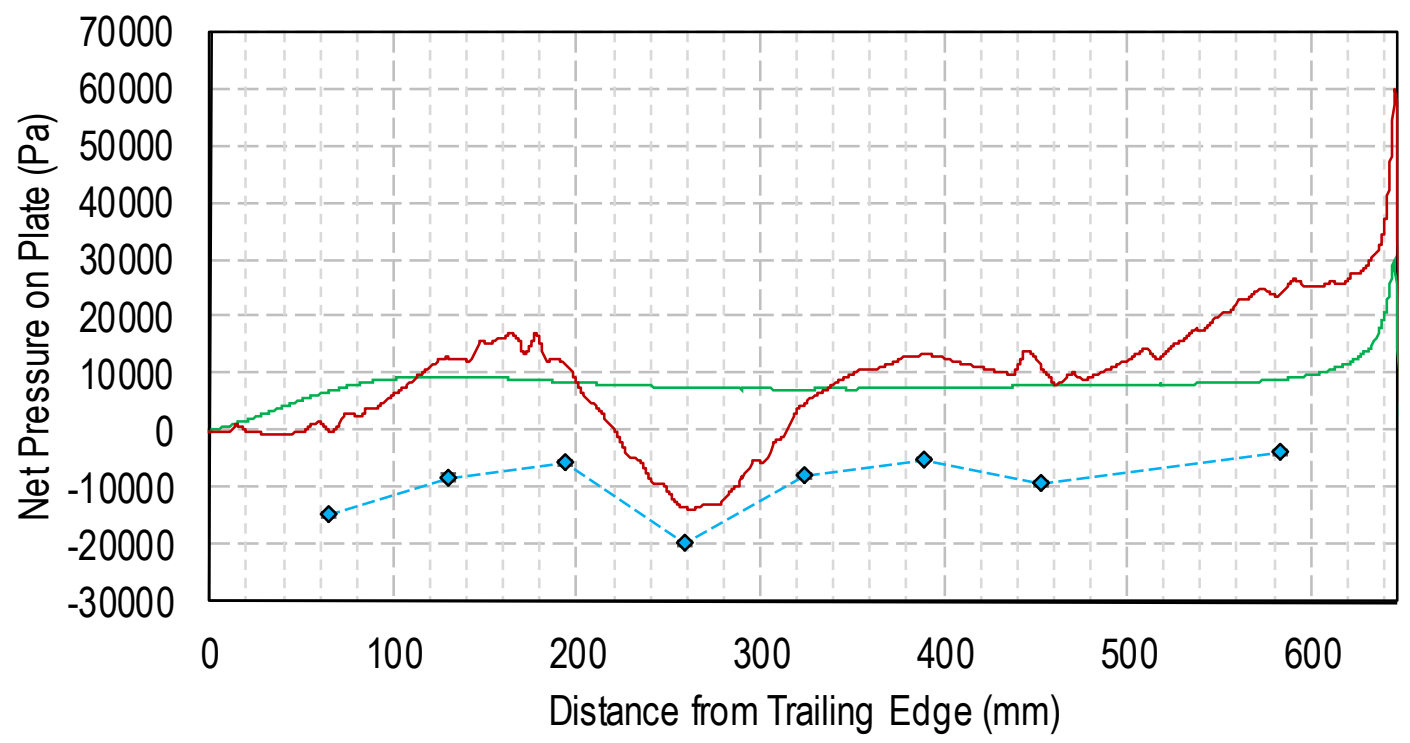

Figure 12.20. $3.43 \mathrm{~kg} / \mathrm{s}$ experiment \& FSI axial pressure results comparison

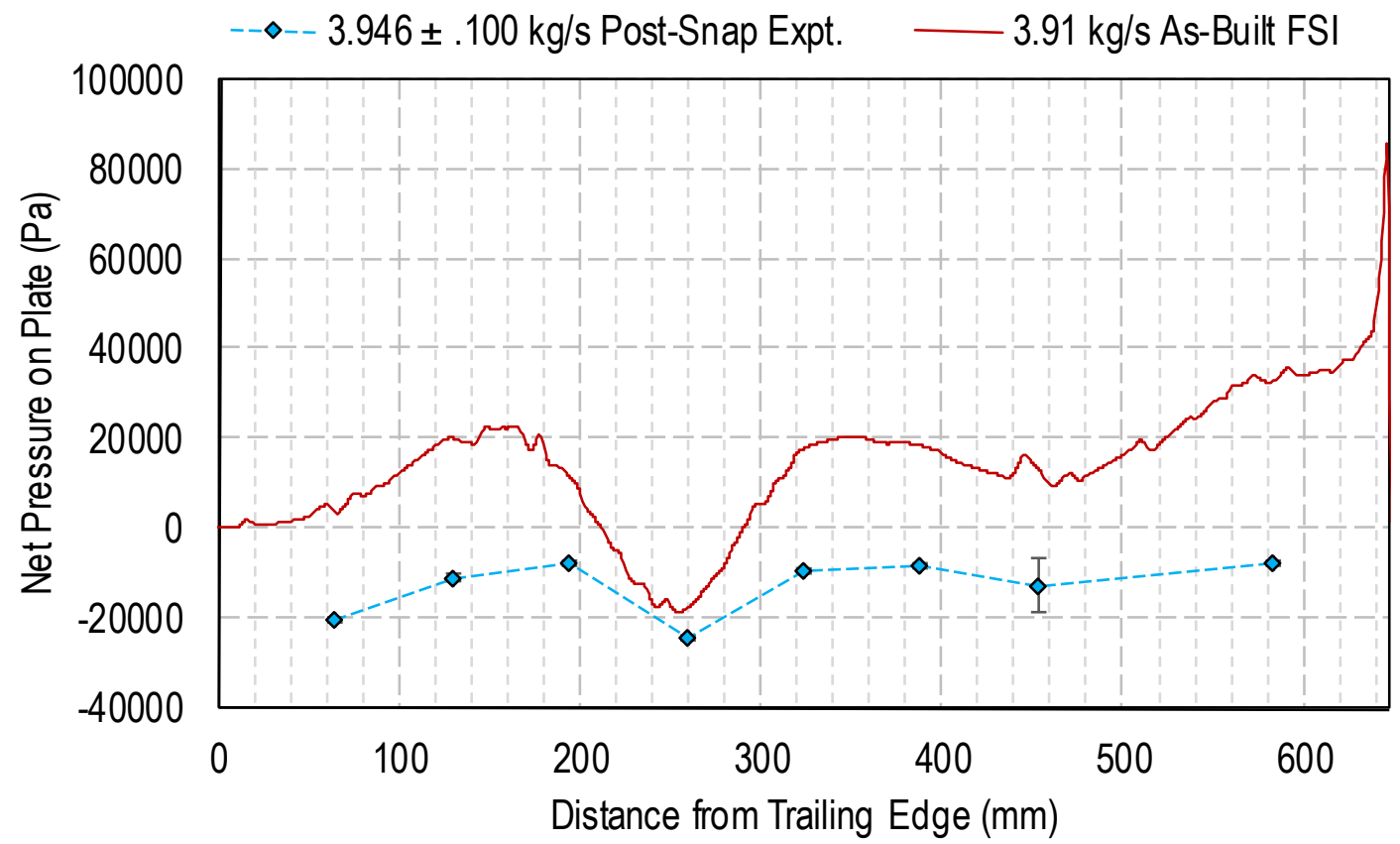

Figure 12.21. $3.91 \mathrm{~kg} / \mathrm{s}$ experiment \& FSI axial pressure results comparison 
$-\diamond-4.376 \pm .093 \mathrm{~kg} / \mathrm{s}$ Post-Snap Expt. $4.34 \mathrm{~kg} / \mathrm{s}$ As-Built FSI

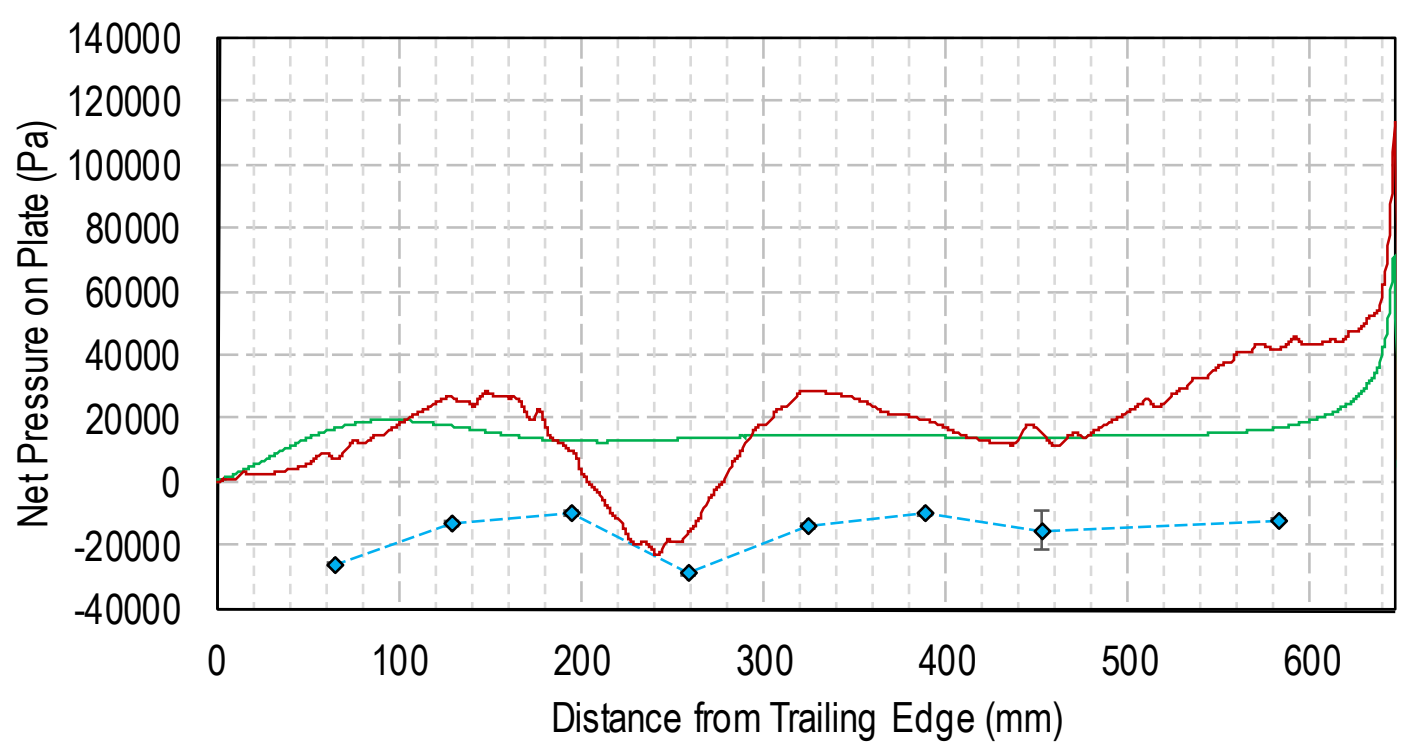

Figure 12.22. $4.34 \mathrm{~kg} / \mathrm{s}$ experiment \& FSI axial pressure results comparison

As with the deflection data, the pressure data from the As-Built simulations matches the pre-snap pressure data from the experiment more closely than the Ideal geometry simulations. Since the plate snap was not captured in the numeric models, the post-snap pressure profiles appear to be offset from the numeric results. The numeric results show generally higher pressure in the smaller channel, while the post-snap experiment results show generally higher pressure in the larger channel. This switch in the pressure distribution in the experiments, which is illustrated well in Figure 12.17, Figure 12.18, and Figure 12.19, clearly shows the pressure force acting on the plate flipping from one channel to the other. 


\section{3: Additional Boundary Condition Improvements}

One potential area where the numeric models could be further improved is in the geometry of the plate. In particular, the shape of the leading edge of the plate. The plate edge in the experiment is likely not square, as it was presented in the numeric models. To test the potential impact of having a non-square plate edge, a series of 2D CFD simulations were completed. These simulations examined three leading edge conditions on a rigid, $1.016 \mathrm{~mm}$ thick plate: square, $45^{\circ} / 0.508 \mathrm{~mm}$ bevel toward the smaller channel, $45^{\circ} / 0.508 \mathrm{~mm}$ bevel toward the larger channel. The resulting meshes for these models are shown in Figure 12.23. All dimensions were based off of the dimensions of Table 4.2, with exception of the width, $b$ (since this is a $2 \mathrm{D}$ study). 


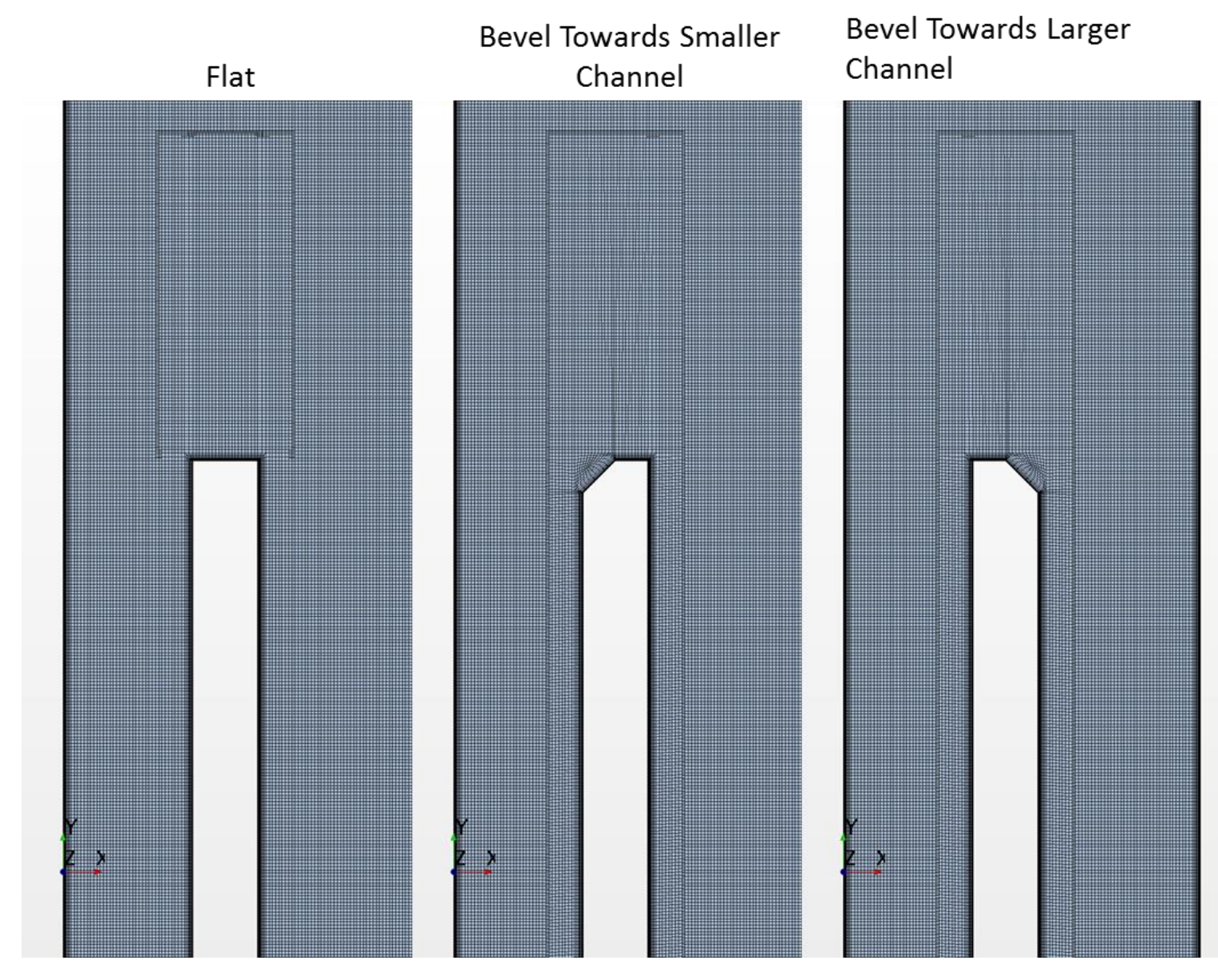

Figure 12.23. 2D leading edge CFD meshes

An average channel velocity of $7.17 \mathrm{~m} / \mathrm{s}$ was selected and the pressure difference of between the two fluid sub-channels was measured in all three cases, as shown in Figure 12.24. Note that the pressure difference is the smaller channel pressure minus the larger channel pressure (i.e. $\left.\Delta \mathrm{P}=\mathrm{P}_{\mathrm{SMALL}}-\mathrm{P}_{\mathrm{LARGE}}\right)$. 


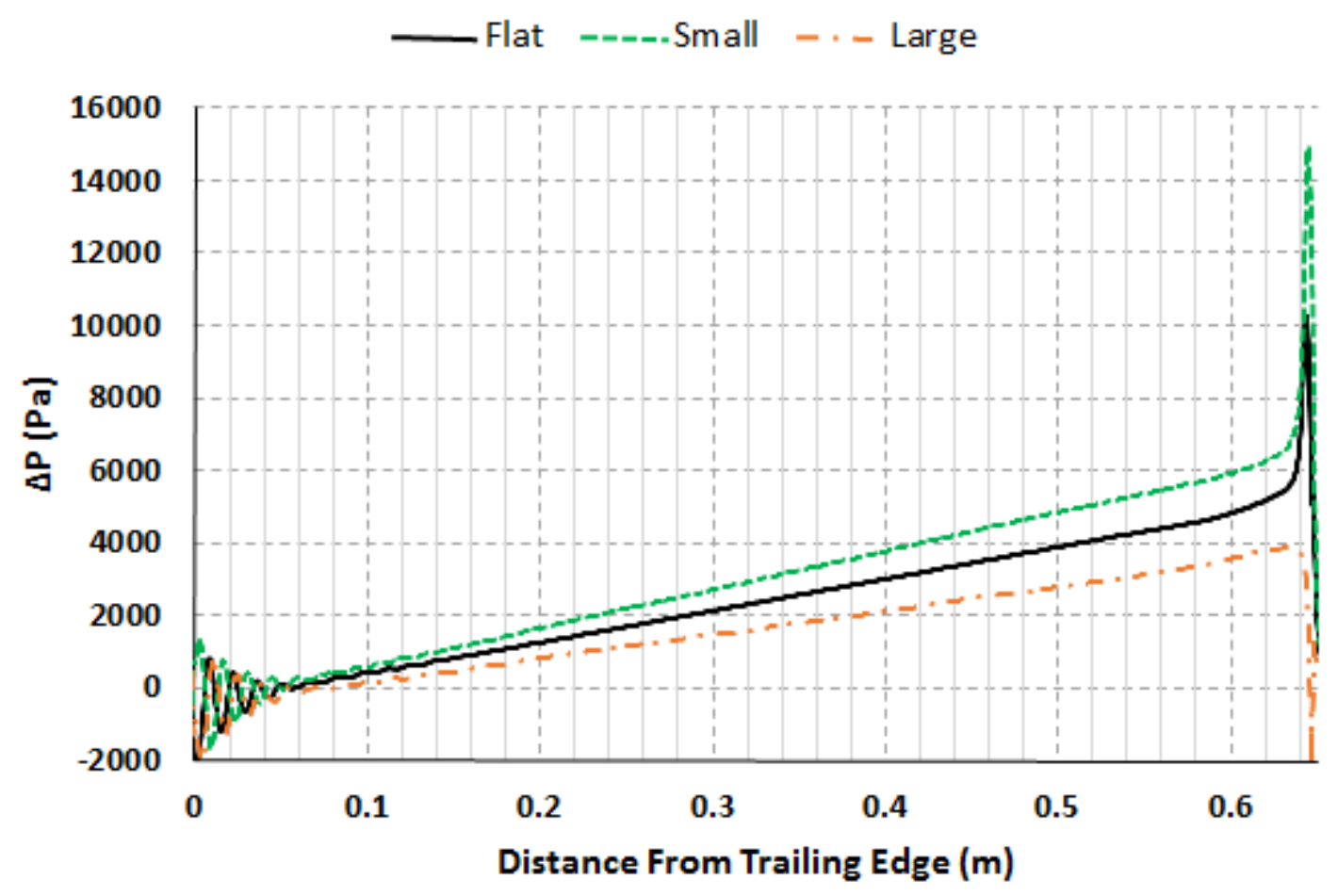

Figure 12.24. 2D edge study pressure results

When compared to the flat bevel, there is a noticeable increase in pressure on the plate when the beveled edge faces toward the smaller channel. Conversely, there is a noticeable decrease in pressure on the plate when the bevel faces toward the larger channel. In the standard case of the flat edge, it is expected that the smaller channel will see a larger pressure acting on the plate. Therefore, if the bevel is directing more flow into a given channel, it makes sense that the bevel toward one channel would result in an increased flow rate to that channel, a decreased flow rate to the other channel, and a significant change in the pressure force acting on the plate.

An additional consideration in assessing the boundary conditions is the clamped condition imposed on the plate. In the numeric model, significant reaction forces are 
noticed in the plane of the plate as it's deflection increases. Assuming these forces are present in the experiment as well, it is possible they are overcoming the static friction clamping force restraining the plate. This possibility also supports the potential for a snap, as the plate could move quite suddenly once the friction force is overcome. At the moment, there are significant obstacles to modeling this phenomenon numerically. These obstacles arise primarily from the changing area of the fluid-structure interface as the plate slides in and out of the side restraints.

\section{4: Conclusions \& Discussion}

The experiment results showed that a flat plate is highly sensitive to even small perturbations in the fluid channel geometry and boundary conditions. This premise was tested numerically through comparison of simulations which used Ideal geometry dimensions, and slightly modified 'As-Built' dimensions which more closely matched the experiment. The idealized dimensions produced results which were consistent with theory. In particular, all plate deflection was into the larger fluid channel, and the maximum deflection was noticed at the leading edge of the plate, where the highest pressure difference was expected. These results, however, failed to replicate the results seen in the experiment. Therefore, the boundary conditions used in the numeric models were re-evaluated, and the As-Built modeling technique was developed.

The As-Built models relied up generation of a detailed map of the experiment fluid channels, and morphing of the Ideal geometry CFD model to match the measurements. With this closer matching of the fluid channel thickness, the numeric model matching of the experiment deflections greatly improved for low to moderate flow rates. However, 
the dynamic plate 'snap' effect was not captured in the numeric models. Therefore, at higher flow rates, the matching between the experiment and As-Built numeric models remained poor.

Additional boundary condition re-evaluations may further improve numeric-experiment matching, particularly at higher flow rates. The significant impact that the shape of the leading edge has on the pressure force experienced by the plate could explain the poor matching seen in most cases near the plate leading edge. There is also significant potential for the plate to be translating in the plane of the plate during flow experiments. At higher flow rates, the in-plane reaction forces may eventually overcome the static friction force of the clamped boundary condition. This would result in the plate slipping from the side support, greatly increasing the deflection seen in the fluid channel. A similar phenomenon has been noted in literature, where clamped plate vibration modes in an experiment at high flow rates appeared converge with modes expected in a pinned edge condition. The loss of the in-plane translation constraint may explain this phenomenon [50]. 


\section{APPENDIX A}

Python beam and plate bending code.

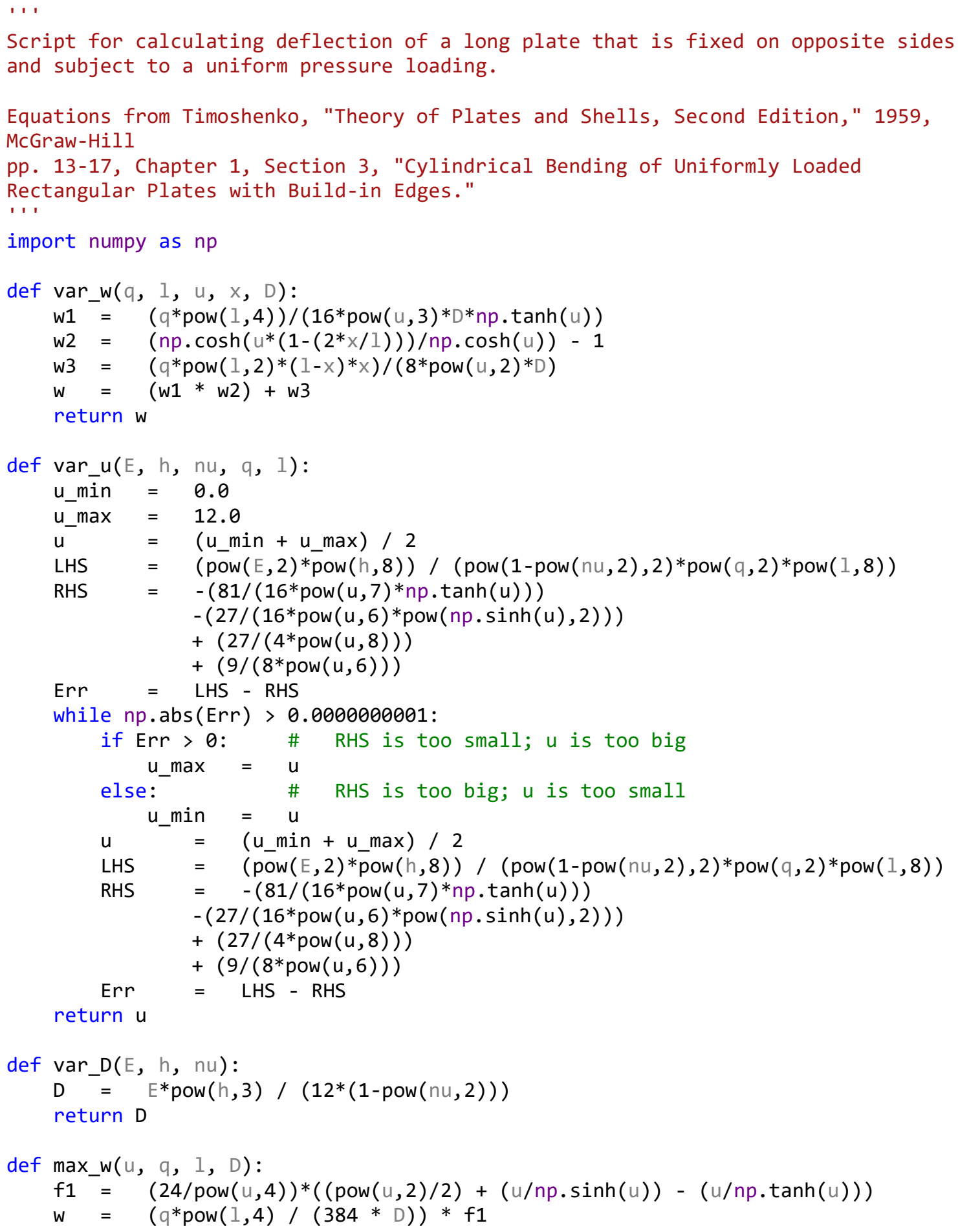




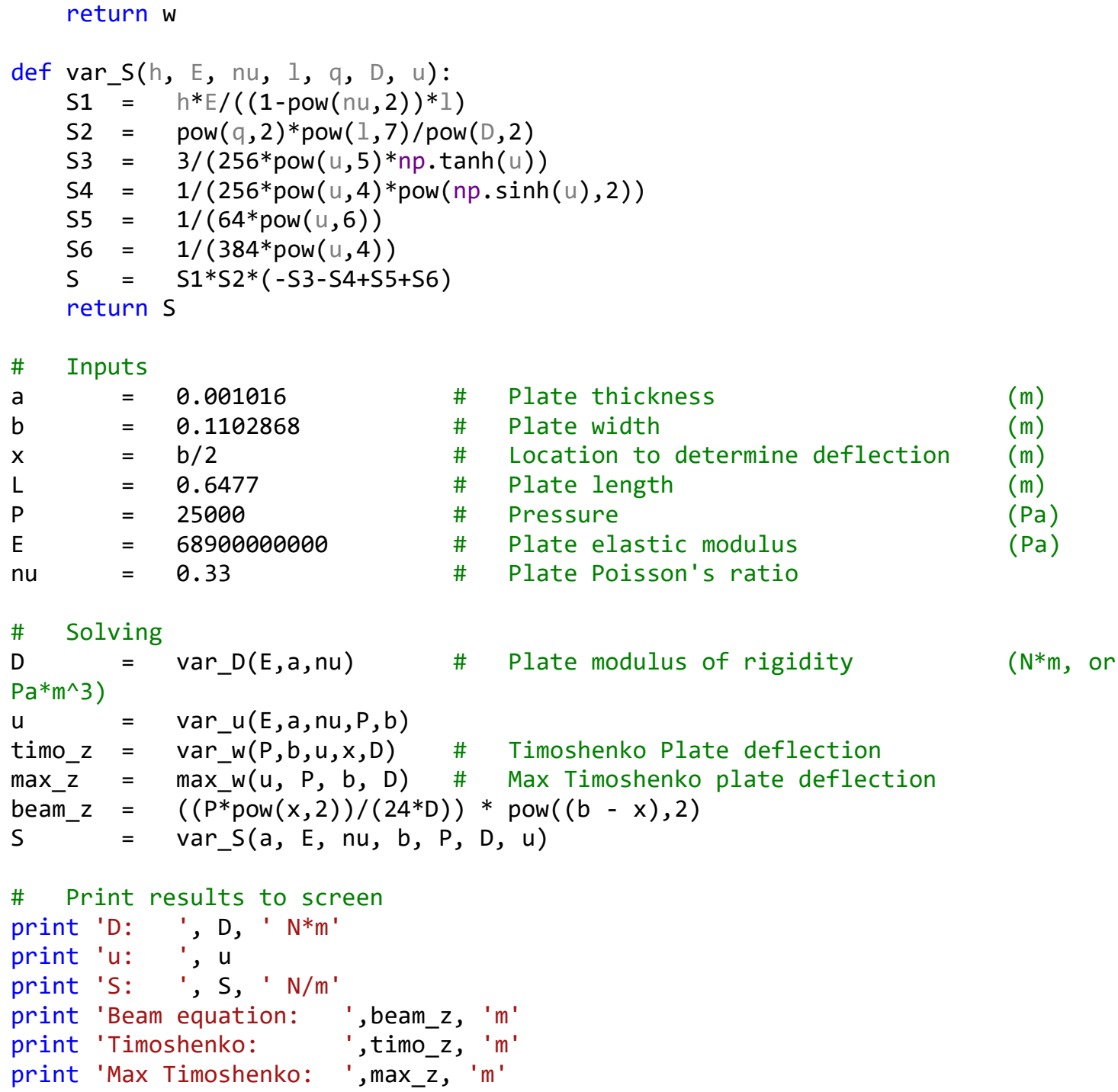




\section{APPENDIX B}

Initial Channel Gaps for Laser Calibration

Free (Un-Pinned) Edges

\begin{tabular}{|c|c|c|}
\hline Laser Location (from trailing edge) & Larger Channel (mils) & Smaller Channel (mils) \\
\hline A - 12.7 mm (Figure 5.5) & 96.819 & 90.410 \\
\hline B - 152.4 mm (Figure 5.6) & 95.431 & 92.626 \\
\hline C - 226.7 mm (Figure 5.7) & 88.266 & 83.213 \\
\hline D - $290.5 \mathrm{~mm}$ (Figure 5.8) & 93.156 & 77.402 \\
\hline E - 474.3 mm (Figure 5.9) & 101.429 & 71.202 \\
\hline F - 550.5 mm (Figure 5.10) & 93.220 & 67.962 \\
\hline G - 635.0 mm (Figure 5.11) & 95.638 & 70.431 \\
\hline
\end{tabular}

Combed (Pinned) Edges

\begin{tabular}{|c|c|c|}
\hline Laser Location (from trailing edge) & Larger Channel (mils) & Smaller Channel (mils) \\
\hline A - 12.7 mm (Figure 5.26) & 93.952 & 88.823 \\
\hline B - 152.4 mm (Figure 5.27) & 93.497 & 92.022 \\
\hline $\mathrm{C}-226.7 \mathrm{~mm}$ (Figure 5.28) & 90.191 & 81.289 \\
\hline D - 290.5 mm (Figure 5.29) & 92.504 & 76.525 \\
\hline E - 474.3 mm (Figure 5.30) & 100.097 & 74.135 \\
\hline F - 550.5 mm (Figure 5.31) & 93.693 & 69.486 \\
\hline G - 635.0 mm (Figure 5.32) & 97.203 & 69.301 \\
\hline
\end{tabular}




\section{APPENDIX C}

Python channel mapping data processing and CFD grid interpolation code.

This code generates plots of the channel mapping data from the experiment and creates

*.CSV files of interpolated data that more closely matches the density of the Star-CCM+ grid. The resulting *.CSV files can be used to modify the ideal geometry outer wall surfaces to match those of the experiment.

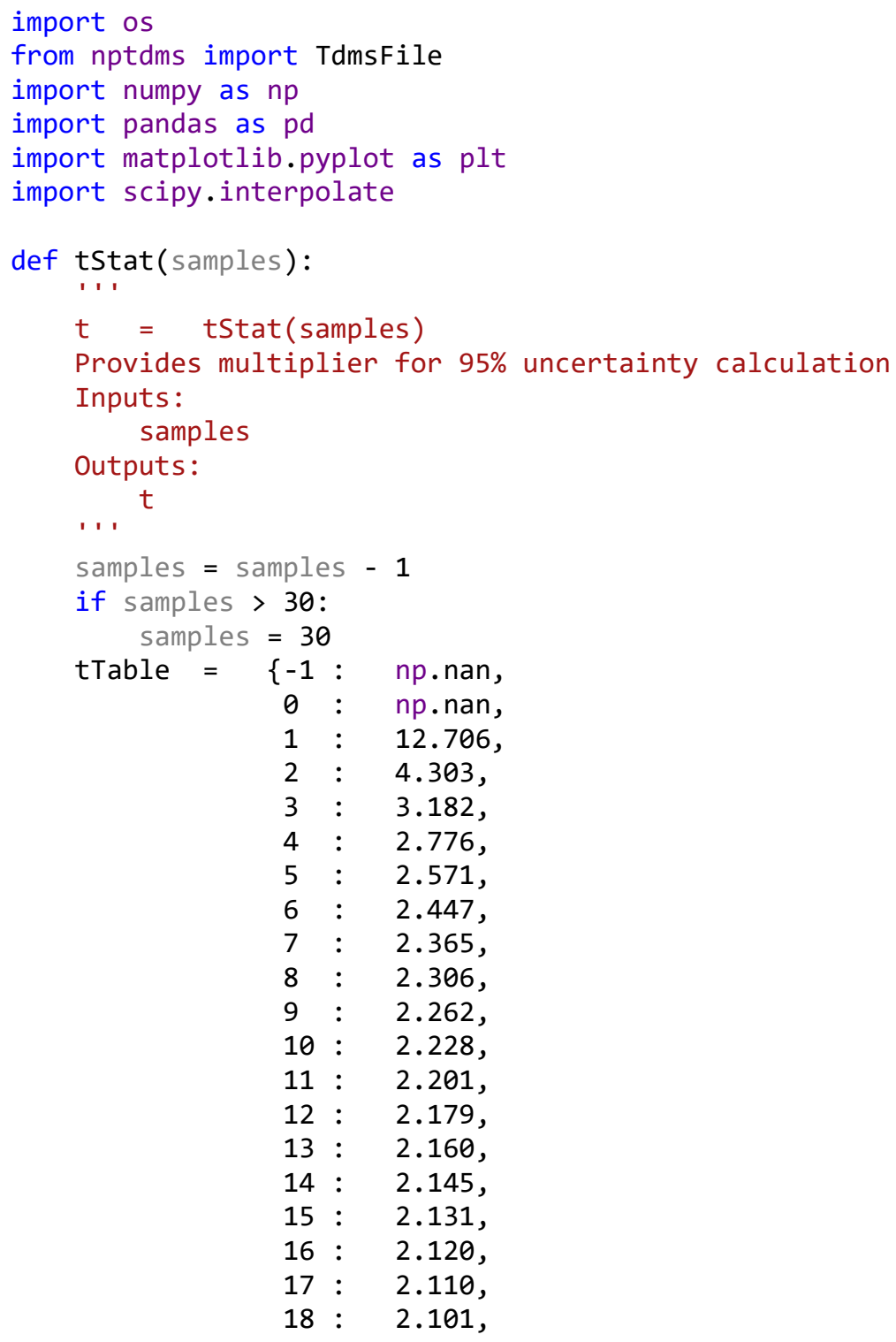




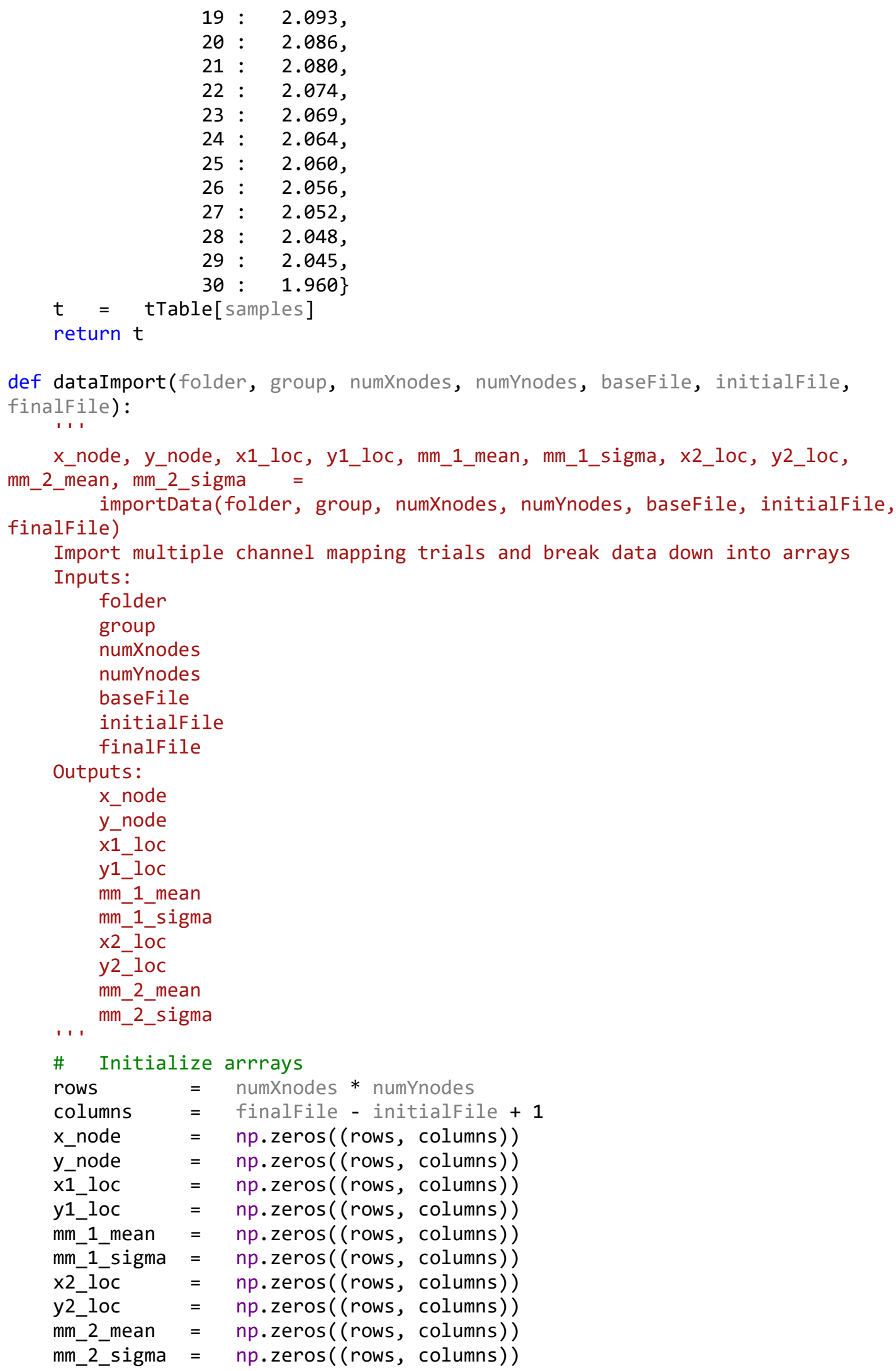




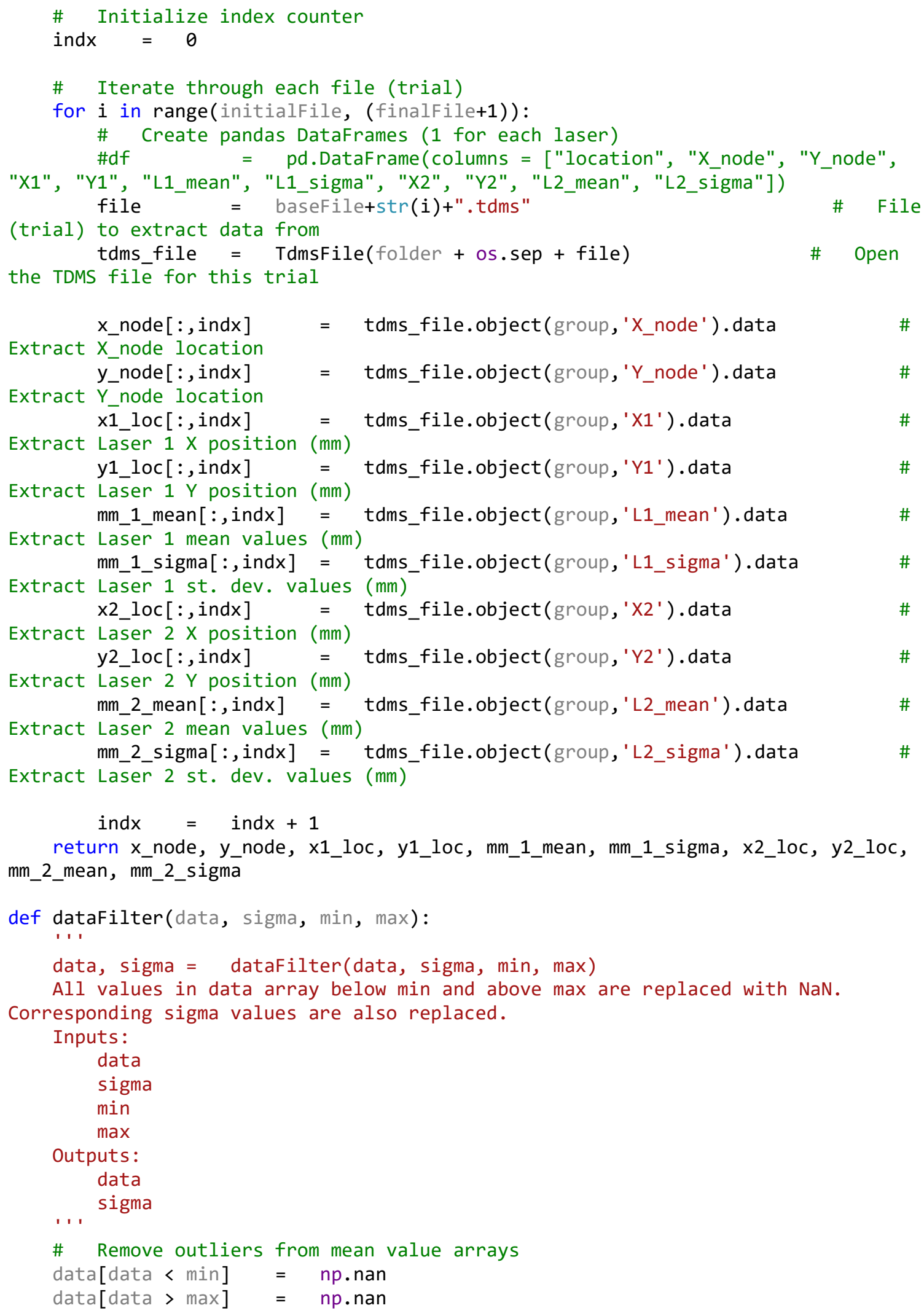




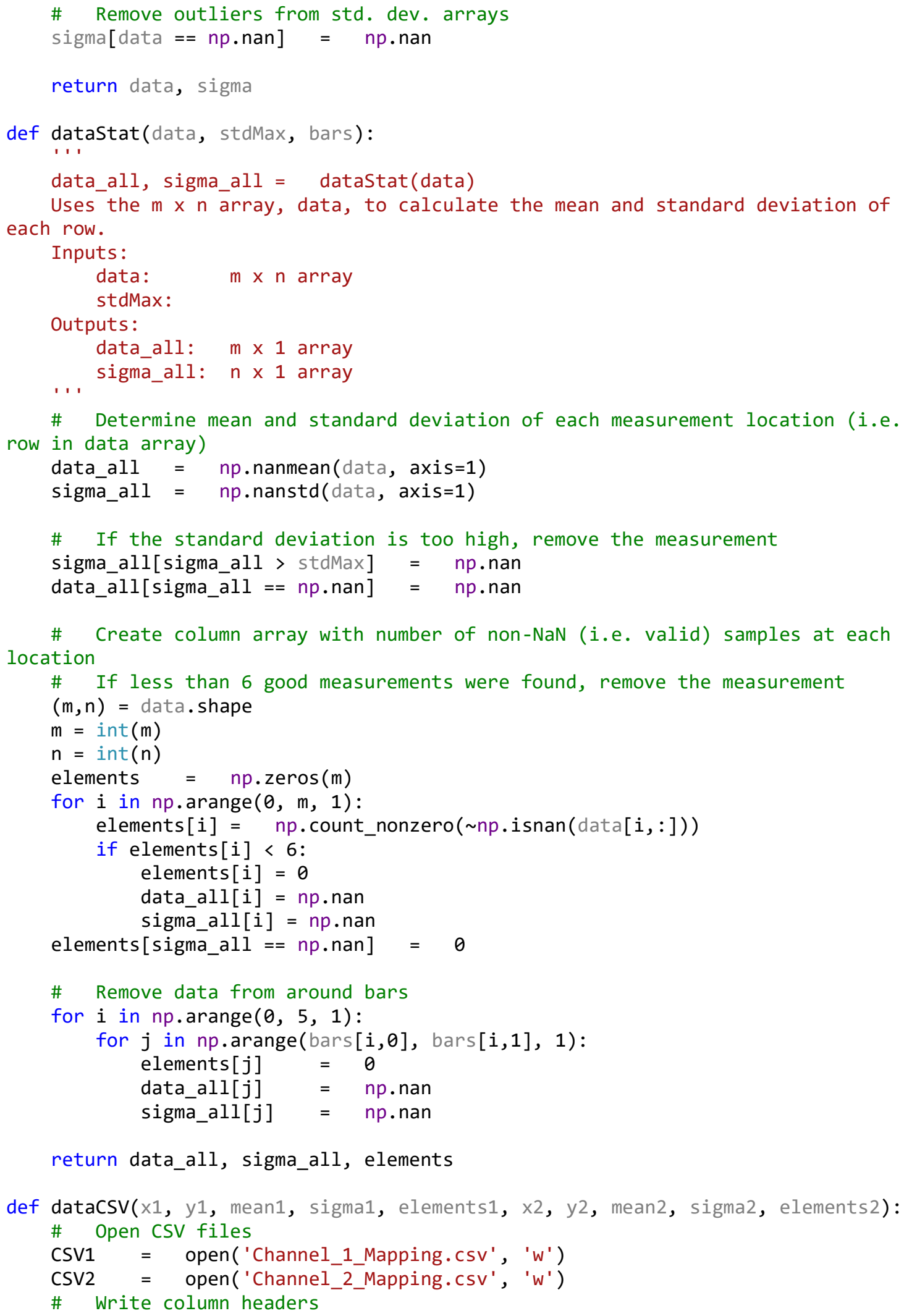




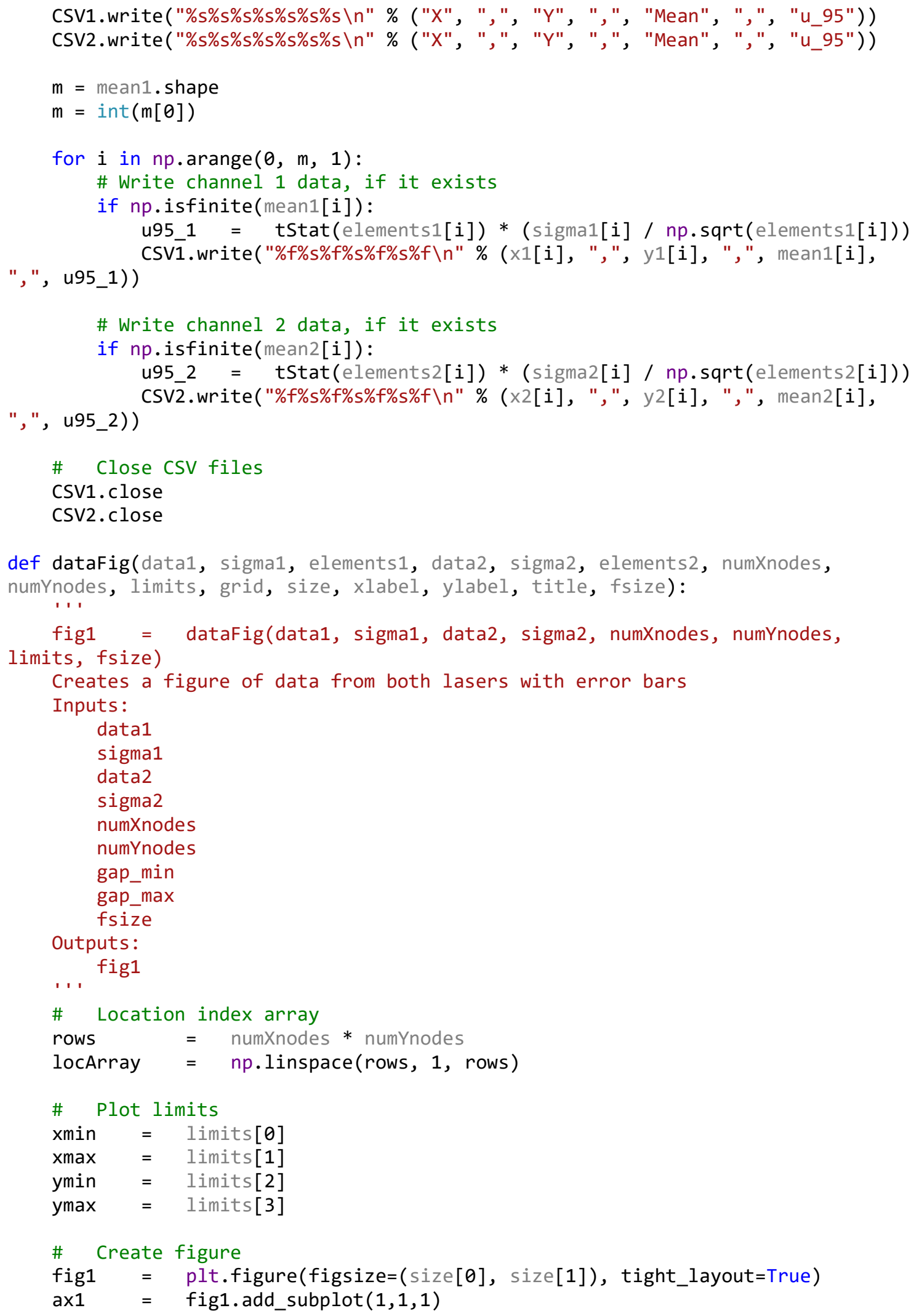


\# Apply limits

ax1.set_xlim(xmin, $x \max )$

ax1.set_ylim(ymin, ymax)

\# Tick locations

ax1.set_xticks(np.arange(xmin, $x$ max+grid[0], grid[0]))

ax1.set_yticks(np.arange(ymin, ymax+grid[1], grid[1]))

\# Tick labels

ax1.set_xticklabels(np.arange(xmin, xmax+grid[0], grid[0]), rotation=90, fontsize=fsize)

ax1.set_yticklabels(np.arange(ymin, ymax+grid[1], grid[1]), fontsize=fsize)

\# Labels

ax1.set_xlabel( $x l a b e l$, fontsize=fsize)

ax1.set_ylabel(ylabel, fontsize=fsize)

ax1.set_title(title, fontsize=fsize)

\# Add grid lines to the plot

for $i$ in $n p . \operatorname{arange}(x \min , x \max , \operatorname{grid}[0])$ :

$X X=[i, i]$

$Y Y=[y m i n, y m a x]$

ax1.plot (XX, YY, "--", lw=0.5, color="black", alpha=0.3)

for $j$ in np.arange(ymin, ymax, grid[1]):

$X X=[x \min , x \max ]$

$Y Y=[j, j]$

ax1.plot(XX, YY, "--", lw=0.5, color="black", alpha=0.3)

\# Add data to the plot

ax1.scatter(locArray, data1, c="Blue", marker="o", alpha=0.5, label="Laser 1")

ax1.scatter(locArray, data2, c="Red", marker="^", alpha=0.5, label="Laser 2")

\# Add error bars

$\mathrm{m} 1$ = data1. shape

$\mathrm{m} 2$ = data2. shape

$\mathrm{m} 1=\operatorname{int}(\mathrm{m} 1[0])$

$m 2=\operatorname{int}(m 2[0])$

u_t1 $=n p \cdot z \cos (\mathrm{m} 1)$

$u_{-} t 2=n p \cdot \operatorname{zeros}(\mathrm{m} 2)$

for $i$ in $n p$. arange $(0, m 1,1)$ :

$u_{-} 1[i]=\operatorname{tStat}($ elements1[i]) $*(\operatorname{sigma1}[i] / \operatorname{np} \cdot \operatorname{sqrt}($ elements1[i]) $)$

for $i$ in $n p$.arange $(0, m 2,1)$ :

u_t2[i] = tStat $($ elements $2[i]) *(\operatorname{sigma2}[i] / \operatorname{np} . \operatorname{sqrt}($ elements2[i]))

ax1.errorbar(locArray, data1, yerr=[u_t1, u_t1], ecolor='b', capthick=1, linestyle="")

ax1.errorbar(locArray, data2, yerr=[u_t2, u_t2], ecolor='r', capthick=1, linestyle=" ")

print 'Laser 1 95\% CI:'

print np.nanmean (u_t1)

print 'Laser 2 95\% CI:'

print np.nanmean(u_t2)

\# Add legend to the plot

leg $=\quad a x 1$.legend $($ loc $=$ 'lower left' $)$

return fig1 


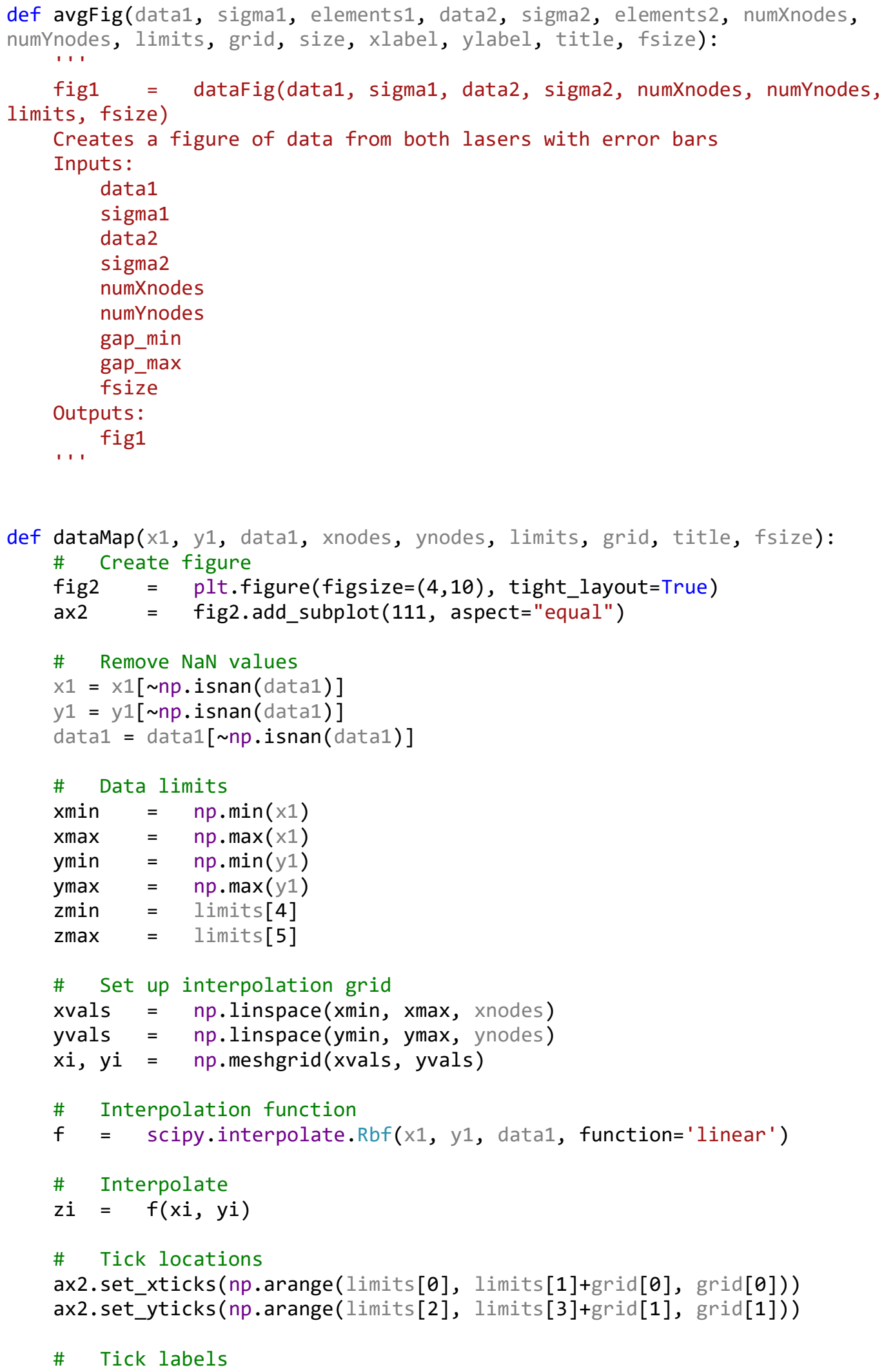


ax2.set_xticklabels(np.arange(limits[0], limits[1]+grid[0], grid[0]), rotation $=90$, fontsize $=$ fsize)

ax2.set_yticklabels(np.arange(limits[2], limits[3]+grid[1], grid[1]), ha=' right' , fontsize=fsize)

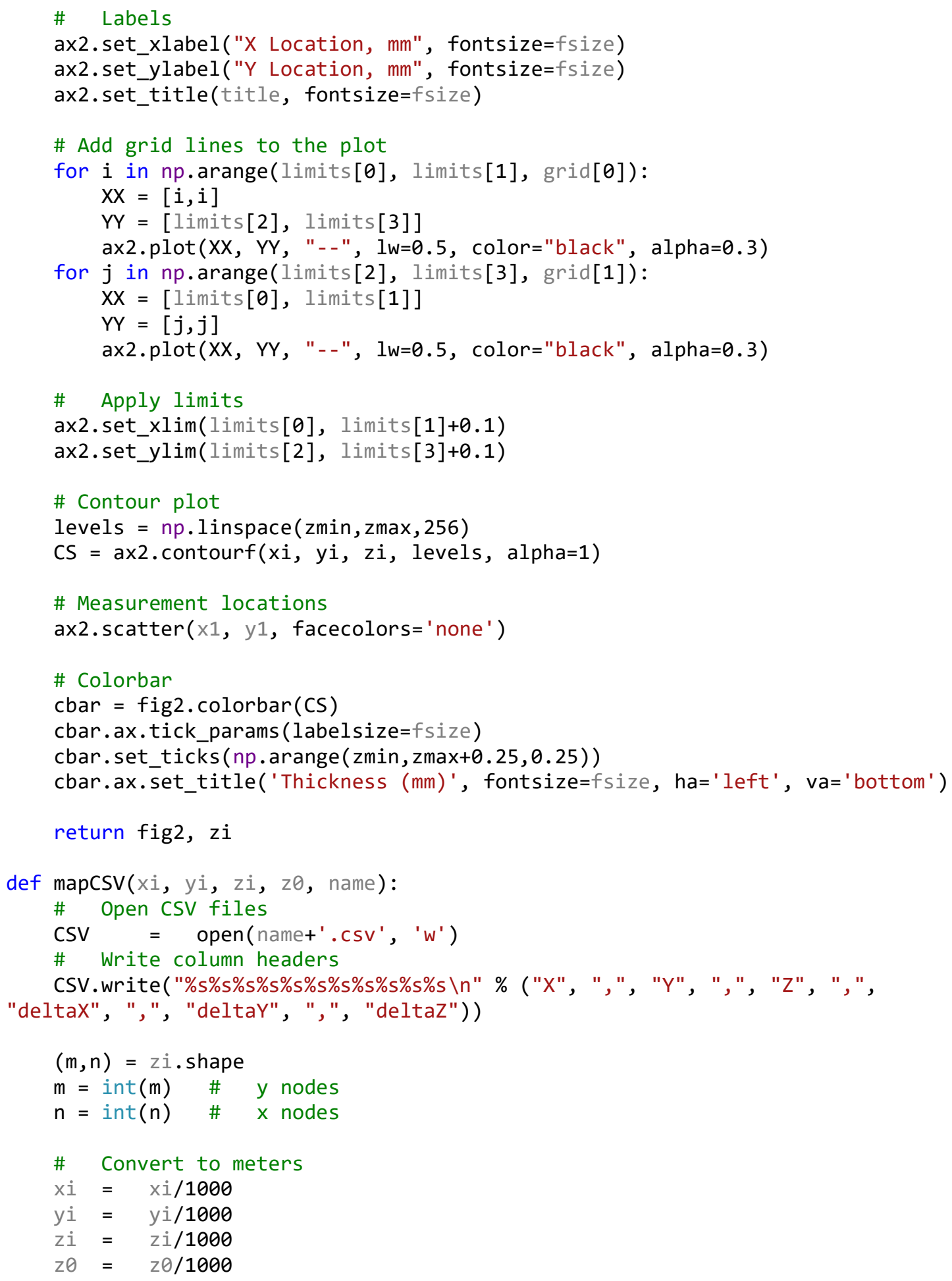




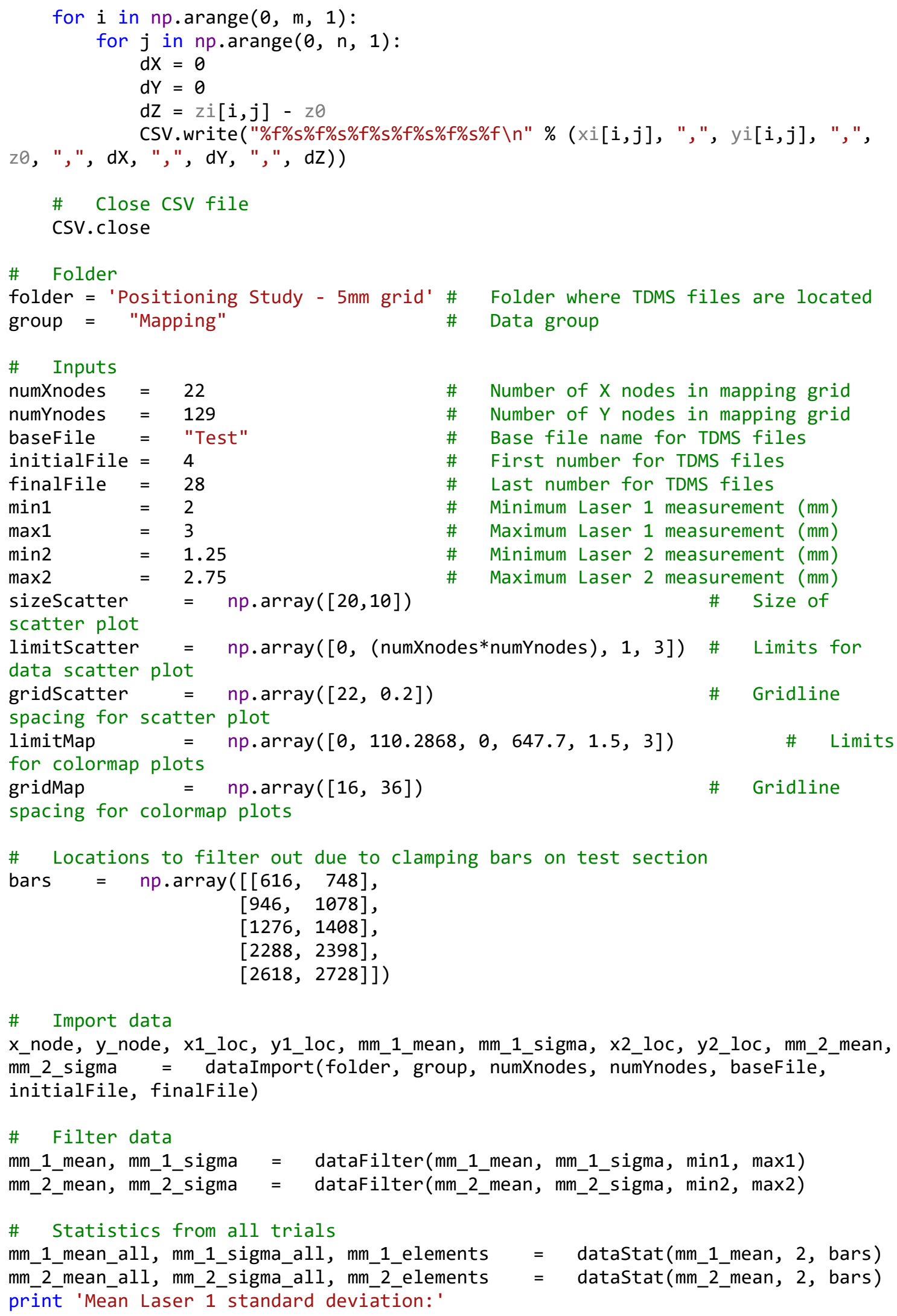


print np.nanmean(mm_1_sigma_all)

print 'Mean Laser 2 standard deviation:'

print np.nanmean(mm_2_sigma_all)

\# Write data to csv

$\operatorname{dataCSV}\left(x 1 \_l o c[:, 0], y 1 \_l o c[:, 0], m m \_1\right.$ mean_all, mm_1_sigma_all, mm_1_elements, $\mathrm{x} 2 \_\operatorname{loc}[:, 0], \mathrm{y} 2 \_10 \mathrm{l}[:, 0], \mathrm{mm}$ 2__mean_all, mm_2_sigma_all, mm_2_elements)

\# Plot data

xlabel = 'Measurement Location'

ylabel = 'Channel Thickness $(\mathrm{mm}){ }^{\prime}$

title $=$ 'Channel Thickness Scatter'

fsize $=10 \quad \#$ Font size for plot

fig1 = dataFig(mm_1_mean_all, mm_1_sigma_all, mm_1_elements, mm_2_mean_all, mm_2_sigma_all, mm_2_e $\bar{l}$ ements, numXnodes, numȲnodes, $\bar{l}$ imitscatter, gridscatter, sizescatter, xlabel, ylabel, title, fsize)

fig1.savefig( 'MappingScatter.png')

\# Plot standard deviations

xlabel = 'Measurement Location'

ylabel = 'Standard Deviation $(\mathrm{mm})$ '

title = 'Measurement Standard Deviation Scatter'

falseSigma1 = np.empty $\left(\mathrm{mm}_{1} 1\right.$ _sigma_all.shape $)$

falseSigma $2=$ np.empty $\left(\mathrm{mm}_{2} 2\right.$ _sigma_all.shape $)$

falseSigma1[:] = np.nan

falseSigma2[:] = np.nan

fig4 = dataFig(mm_1_sigma_all, falseSigma1, mm_1_elements, mm_2_sigma_all, falseSigma2, mm_2_elements, numXnodes, numYnodes, np.array $([0,2838,0,0.4])$,

[22, 0.02], sizescatter, xlabel, ylabel, title, fsize)

\# Plot number of good measurements

xlabel = 'Measurement Location'

ylabel = 'Measurement Samples'

title = 'Valid Measurement Samples at Each Location'

falseSigma1 = np.empty $\left(\mathrm{mm}_{-}\right.$1_elements.shape $)$

falseSigma2 $=$ np.empty $\left(\mathrm{mm}_{2}\right.$ 2_elements.shape $)$

falseSigma1[:] = np.nan

falseSigma2[:] = np.nan

fig5 = dataFig(mm_1_elements, falseSigma1, mm_1_elements, mm_2_elements, falseSigma2, mm_2_elements, numXnodes, numYnodes, np.array $([0,2838,0,25]),[22$, 1], sizeScatter, xlabel, ylabel, title, fsize)

fig5.savefig('ValidMeasurements.png')

\# Star-CCM+ grid

starXnodes $=$ int $(110.2868 / 1.27)$

starYnodes $\quad=\quad$ int $(647.7 / 1.27)$

\#starXnodes $\quad=$ numXnodes

\#starYnodes $\quad=$ numYnodes

\# Generate colormaps

fig2, zi_1 = dataMap $\left(x 1\right.$ loc $[:, 0], y 1 \_l o c[:, 0]$, mm_1_mean_all, starXnodes, starYnodes, limitMap, gridMap, "Channel 1 - Fine Mapping", fsize)

fig2.savefig( 'Channel_1_Fine.png')

fig3, zi_2 = dataMap $\left(x 2 \_l o c[:, 0], y 2 \_l o c[:, 0]\right.$, mm_2_mean_all, starXnodes, starYnodes, limitMap, gridMap, "Channel 2 - Fine Mapping", $\bar{f}$ size) 


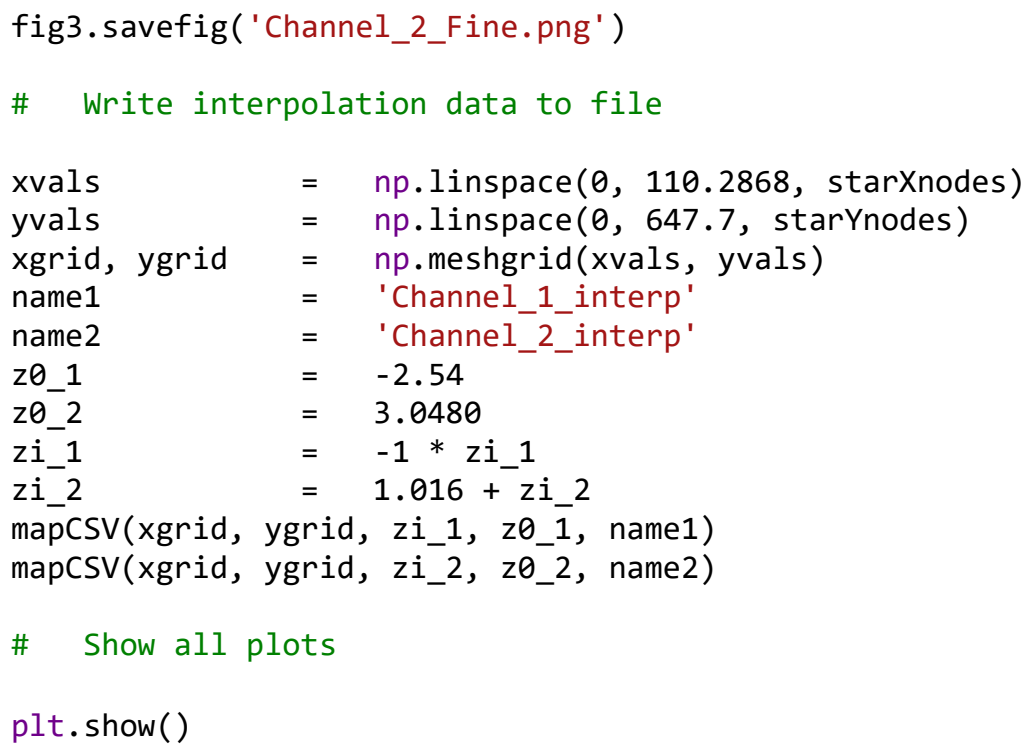




\section{REFERENCES}

[1] University of Missouri Research Reactor, "MU Research Reactor," University of Missouri, [Online]. Available: http://www.murr.missouri.edu. [Accessed 2509 2014].

[2] J. C. McKibben and J. G. Stevens, "Feasibility Analyses for HEU to LEU Fuel Conversion of the University of Missouri Research Reactor (MURR)," University of Missouri Research Reactor, Columbia, MO, 2009.

[3] E. E. Feldman, "Technical Basis in Support of the Conversion of the University of Missouri Research Reactor (MURR) Core from Highly-Enriched to Low-Enriched Uranium - Steady-State Thermal-Hydraulics Analysis," Argonne National Laboratory, Argonne, IL, 2012.

[4] NNSA, "Office of Global Threat Reduction," 2011. [Online]. Available: http://nnsa.energy.gov/aboutus/ourprograms/nonproliferation/programoffices/office globalthreatreduction. [Accessed 25 June 2011].

[5] J. Stillman, E. Feldman, L. Foyto, K. Kutikkad, J. C. McKibben, N. Peters and J. Stevens, "Technical Basis in Support of the Conversion of the University of Missouri Research Reactor (MURR) Core from Highly-Enriched to Low-Enriched 
Uranium - Core Neutron Physics (ANL/RERTR/TM-12-30)," Argonne National Laboratory, Argonne, IL, 2012.

[6] D. E. Burkes, R. Prabhakaran, J.-F. Jue and F. J. Rice, "Mechanical Properties of DU-xMo Alloys with $\mathrm{x}=7$ to 12 Weight Percent," Metallurgical and Materials Transactions A, vol. 40, no. A, pp. 1069-1079, 2009.

[7] D. E. Burkes, C. A. Papesch, A. P. Maddison, T. Hartman and F. J. Rice, "Thermophysical properties of DU-10 wt.\% Mo alloys," Journal of Nuclear Materials, vol. 403, pp. 160-166, 2010.

[8] United States of America Department of Defense, Metallic Materials and Elements for Aerospace Vehicle Structures MIL-HDBK-5H, Washington, D.C., 1998.

[9] M. B. Waldron, R. C. Burnett and S. F. Pugh, "The Mechanical Properties of Uranium-Molybdenum Alloys," United Kingdom Atomic Energy Research Authority - Atomic Energy Research Establishment, Harwell, Berkshire, 1958.

[10] H. B. Fairchild, "The Properties of Zirconium and its Possibilities for Thermal Reactors," Oak Ridge National Laboratory, Oak Ridge, TN, 1949.

[11] D. R. Miller, "Critical Flow Velocities for Collapse of Reactor Parallel-Plate Fuel Assemblies," Knolls Atomic Power Laboratory, Schenectady, New York, 1958. 
[12] P. Jensen and W. R. Marcum, "Predicting critical flow velocity leading to laminate plate collapse - flat plates," Nuclear Engineering and Design, vol. 267, pp. 71-87, 2014.

[13] W. R. Marcum, "Predicting critical flow velocity leading to laminate plate collapse - Cylindrical plates," Nuclear Engineering and Design, vol. 278, pp. 50-63, 2014.

[14] S. Timoshenko, Theory of Plates and Shells, Second Ed., New York: McGraw-Hill, 1959.

[15] W. C. Roark, Formulas for Stress and Strain, New York: McGraw-Hill, 2001.

[16] R. D. Blevins, Applied Fluid Dynamics Handbook, New York: Van Nostrand Reinhold Company Inc., 1984.

[17] I. E. Idelchik, Handbook of Hydraulic Resistance, 3rd Edition, New York: CRC Press, Inc., 1994.

[18] C. F. Colebrook, "Turbulent Flow in Pipes, with particular reference to the Transition Region between Smooth and Rough Pipe Laws," Journal of the Institution of Civil Engineers, vol. 11, no. 4, pp. 133-156, 1939.

[19] H. Schlichting, Boundary-Layer Theory, New York: McGraw-Hill, 1979. 
[20] W. L. Zabriskie, "An Experimental Evaluation of the Critical Flow Velocity Formulas for Parallel Plate Assemblies," General Electric General Engineering Laboratory, Schenectady, NY, 1958.

[21] W. L. Zabriskie, "An Experimental Evaluation of the Effect of Length-to-Width Ratio on the Critical Flow Velocity of Single Plate Assemblies," General Electric General Engineering Laboratory, Schenectady, NY, 1959.

[22] R. D. Groninger and J. J. Kane, "Flow Induced Deflections of Parallel Flat Plates," Nuclear Science and Engineering, no. 16, pp. 218-226, 1963.

[23] G. E. Smissaert, "Static and Dynamic Hydroelastic Instabilities in MTR-Type Fuel Elements: Part I. Introduction and Experimental Investigation," Nuclear Engineering and Design, vol. 7, no. 6, pp. 535-546, 1968.

[24] W. F. Swinson, R. L. Battiste, C. R. Luttrell and G. T. Yahr, "Fuel Plate Stability Experiments and Analysis for the Advanced Neutron Source," Symposium on Flow-Induced Vibration and Noise, vol. 244, pp. 133-143, 1992.

[25] W. F. Swinson, R. L. Battiste, C. R. Luttrell and G. T. Yahr, "Structural Response of Reactor Fuel Plates to Coolant Flow," PVP, Flow-Induced Vibration and FluidStructure Interaction, ASME, vol. 258, pp. 21-33, 1993. 
[26] W. F. Swinson, R. L. Battiste, L. R. Luttrell and G. T. Yahr, "An Experimental Investigation of the Structural Response of Reactor Fuel Plates," Journal of Experimental Mechanics, no. 9, pp. 212-215, 1995.

[27] M. Ho, G. Hong and A. Mack, "Experimental Investigation of Flow-Induced Vibration in Parallel Plate Reactor Fuel Assembly," in 15th Australasian Fluid Mechanics Conference, Sydney, Australia, 2004.

[28] L. Liu, D. Lu, Y. Li, P. Zhang and F. Niu, "Large-amplitude and narrow-band vibration phenomenon of a foursquare fix-supported flexible plate in a rigid narrow channel," Nuclear Engineering and Design, vol. 241, no. 8, pp. 2874-2880, 2011.

[29] Y. Li, D. Lu, P. Zhang and L. Liu, "Experimental investigation on fluid-structure interaction phenomenon caused by the flow through double-plate structure in a narrow-channel," Nuclear Engineering and Design, vol. 248, pp. 66-71, 2012.

[30] O. Reynolds, "On the Dynamical Theory of Incompressible Viscous Fluids and the Determination of the Criterion," Proceedings of the Royal Society of London, no. 56, pp. 40-45, 1894.

[31] D. C. Wilcox, Turbulence Modeling for CFD, La Canada, California: DCW Industries, Inc., 1994.

[32] D. C. Wilcox, "Formulation of the k- $\omega$ Turbulence Model Revisited," AIAA Journal, vol. 46, no. 11, pp. 2823-2838, 2008. 
[33] F. R. Menter, "Two-Equation Eddy-Viscosity Turbulence Models for Engineering Applications," AIAA Journal, vol. 32, no. 8, pp. 1598-1605, 1994.

[34] P. H. Saksono, W. G. Dettmer and D. Peric, "An adaptive remeshing strategy for flows with moving boundaries and fluid-structure interaction," International Journal for Numerical Methods in Engineering, vol. 71, pp. 1009-1050, 2007.

[35] R. L. Meakin, "Composite Overset Structured Grids," in Handbook of Grid Generation, Boca Raton, FL, CRC Press, 1999, p. Chapter 11.

[36] E. Schreck, M. Peric and D. Snyder, "Overset Grids Technology in STAR-CCM+: Methodology and Applications," in 11th Symposium on Overset Composite Grids and Solution Technology, Dayton, OH, 2012.

[37] M. Razzaq, S. Turek, J. Hron, J. F. Acker, F. Weichert, I. Q. Grunwald, C. Roth, M. Wagner and B. F. Romeike, "Numerical Simulation and Benchmarking of Fluid-Structure Interaction with Application to Hemodynamics," in Fundamental Trends in Fluid-Structure Interaction, Singapore, World Scientific Publishing, 2010, pp. 171-199.

[38] Y. Bazilevs, K. Takizawa and T. E. Tezduyar, Computational Fluid-Structure Interaction: Methods and Applications, West Sussex, UK: John Wiley \& Sons, 2013. 
[39] P. Causin, J.-F. Gerbeau and F. Nobile, "Added-mass effect in the design of partitioned algorithms for fluid-structure problems," INRIA, Paris, 2004.

[40] B. Roe, A. Haselbacher and P. H. Geubelle, "Stability of fluid-structure thermal simulations on moving grids," International Journal for Numerical Methods in Fluids, vol. 54, no. 1, pp. 1097-1117, 2007.

[41] P. J. Roache, "Quanitification of Uncertainty in Computational Fluid Dynamics," Annual Review of Fluid Mechanics, vol. 29, no. 1, pp. 123-160, 1997.

[42] I. B. Celik, U. Ghia, P. J. Roache, C. J. Freitas, H. Coleman and P. E. Raad, "Procedure for Estimation and Reporting of Uncertainty Due to Discretization in CFD Applications," Journal of Fluids Engineering, vol. 130, no. 7, 2008.

[43] K. K. Turner, The Thermal-Mechanical Analysis of Targets for the High Volume Production of Molybdenum-99 Using a Low-Enriched Uranium Metal Foil (Dissertation), Columbia, MO: University of Missouri, 2012.

[44] J. C. Kennedy, "Hydro-Mechanical Analysis of Low Enriched Uranium Fuel Plates for University of Missouri Research Reactor," University of Missouri, Columbia, MO, 2012.

[45] J. Stillman, E. Feldman, L. Foyto, K. Kutikkad, J. C. McKibben, N. Peters and J. Stevens, "Conceptual Design Parameters for MURR LEU U-Mo Fuel Conversion 
Design Demonstration Experiment (ANL/RERTR/TM-12-38)," Argonne National Laboratory, Argonne, IL, 2013.

[46] L. Foyto, K. Kutikkad, J. C. McKibben, N. Peters, G. Solbrekken, E. Feldman, J. Stevens, J. Stillman and C. Tzanos, "Preliminary Safety Analysis Report Methodologies and Scenarios for LEU Fuel Conversion of the University of Missouri Research Reactor (MURR)," University of Missouri Research Reactor and Argonne National Laboratory, Columbia, MO, 2011.

[47] T. -H. Shih, W. W. Liou, A. Shabbir, Z. Yang and J. Zhu, "A New k- $\varepsilon$ Eddy Viscosity Model for High Reynolds Numer Turbulent Flows - Model Development and Validation," NASA Technical Memorandum, Cleveland, Ohio, 1994.

[48] C. J. Jesse, J. C. Kennedy, G. D. Schnieders and G. L. Solbrekken, "Stabilization Techniques for Simulation of High-Aspect Ratio Fluid Structure Interaction (FSI) Models of Flat Plates," in American Nuclear Society Winter Meeting, Anaheim, CA, 2014.

[49] P. Causin, J. F. Gerbeau and F. Nobile, "Added-mass effect in the design of partitioned algorithms for fluid-structure problems," Computer methods in applied mechanics and engineering, no. 194, pp. 4506-4527, 2005.

[50] E. H. Dowell, Aeroelasticity of plates and shells, Leyden, The Netherlands: Noordhoff International Publishing, 1975. 
[51] CD-Adapco, "Star-CCM+ 9.04.009-R8," CD-Adapco, Melville, NY, 2014.

[52] Dassault Systems Simulia, "Abaqus 6.13-3," Boston, 2014.

[53] S. V. Patankar, Numerical Heat Transfer and Fluid Flow, New York: Routledge, 1980. 


\section{VITA}

John Kennedy is from Kansas City, MO. After graduating with a B.S. Degree in Mechanical Engineering from the University of Missouri in December 2008, he started working toward a M.S. degree in Mechanical \& Aerospace Engineering at the University of Missouri in the fall of 2009. Working under the guidance of Dr. Gary Solbrekken, John completed his M.S. degree in May of 2012. His M.S. thesis focused on early numeric model development for coupling CFD and FEA applications for Fluid-Structure Interaction (FSI) analysis. Upon completion of his M.S. degree, John began work on his Ph.D. degree in Mechanical \& Aerospace Engineering. His Ph.D. dissertation focused on development of numeric modeling techniques for improvement of numeric model matching to experimental data. He also completed extensive experimental analysis of a flat plate under flow, which is of interest in nuclear fuel analysis. John had the opportunity to work at Argonne National Lab in the summers of 2010 and 2011. After completing his M.S. degree, he went to Seattle in the summers of 2012 and 2013 as a fluid-structure interaction intern for TerraPower. He has written and presented papers at numerous technical conferences, and is in the process of completing publication of several journal articles. 